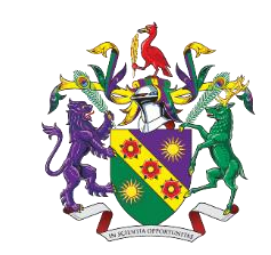

Edge Hill University

\title{
An IoT Approach to \\ Personalised Remote Monitoring and Management of Epilepsy
}

\author{
Sarah Ann McHale \\ Department of Computer Science
}

March 2020

A THESIS SUBMITTED IN PARTIAL FULFILLMENT OF THE REQUIREMENTS OF EDGE HILL UNIVERSITY FOR THE DEGREE OF DOCTOR OF PHILOSOPHY 


\section{Acknowledgments}

I am grateful to the individuals that participated in the clinical studies described in this thesis. I would like to thank them for their patience, generosity, and courage.

Thanks to Dr. Udo Weishmann his mentorship and input while I carried out this project; our initial fortuitous meeting was to make-way for this influential collaboration. Thanks, Udo, for sharing the passion to help epilepsy patients which has motivated us throughout. Thanks to Becky Higginbottom and Amy Ibbotson, and all the Clinical Physiologists' at the Walton Centre for assisting me with patient profile collection and teaching me valuable epilepsy terminology and the methods at the Neurophysiology Department.

Thanks to the NRES committee London-Dulwich (HEALTH RESEARCH AUTHORITY) for supporting me in the design of clinical studies, and for helping me with the NHS IRAS (Integrated Research Approval System). Thanks to Dave Watling for making the ethical aspects of the studies at The Walton Centre possible. The App system used in these studies could not have been built without the help of my colleague and fellow $\mathrm{PhD}$ researcher Oladotun Omosebi: his determination, positivity and skill will long live in the memory. I am hugely thankful for the input from my supervisory team; Professor Nik Besis, Dr. Mark Hall, Dr. Nemitari Ajienka, Dr. Quanbin Sun and Dr.Hui Fang.

I am forever appreciative of my DoS Professor Ella Pereira who, with her expertise, has helped me grow as a computer scientist and researcher over the course of my $\mathrm{PhD}$ career. Ella's commitment, encouragement and support has been unfailing and without an excellent supervisor like her I would not have surpassed the hurdles. It has been a long journey and having Ella by my side has been an honour and pleasure.

Thanks to my husband Mike for being there and supporting me throughout the years of my $\mathrm{PhD}$, for his constant kindness and time; and for our endless discussions about epilepsy and sharing experiences about his epilepsy patients.

Thanks to my mum for always encouraging me to do better, and for always believing in me. Finally, an extraordinary thanks to my Dad who uncannily due to his own epileptic episodes was to inspire me from the beginning do some good for epilepsy by using the power of Computer Science. 


\section{Abstract}

Living with the unpredictable nature of epilepsy is difficult. Not knowing when the next seizure will occur is grim enough, moreover when combined with further complexities associated with the disease epilepsy can become debilitating and often fatal.

Many current studies of epileptic seizure management disclose that an ultimate solution to drugresistant epilepsy is still lacking. Also, although a variety of epilepsy devices exist the prevailing knowledge still resounds that epilepsy is not easily managed, this being due to its sheer complex nature.

An emerging approach is to personalise healthcare and this is known to be facilitated by the Internet of Things (IoT). Therefore, focusing upon personalised parameters that make epilepsy patients distinct from each other this thesis proposes that with IoT technologies there is a more accurate and refined way of remotely monitoring and managing the 'individual' patient. This is achieved by using classification techniques such as ontology development tools and clustering analysis to develop a Patient Profile Description Language (PPDL) and generate meaningful groups to categorize epilepsy patients. Ultimately a monitoring framework is developed to capture this personalised seizure data obtained from an IoT sensor-based device, which is positioned on different parts of the patient's body.

This thesis discloses that it is 'the individual profile' that makes the difference in which IoT sensor-based device to choose and therefore the results used from this study are used to form a typical model or a PMP (Personalised Monitoring Plan) which recommends which IoT sensorbased device to use based on those very individual, personal characteristics of a given patient.

By integrating IoT sensor-based devices deployed remotely and personalised patient data into a combined monitoring framework the vision of personalisation is realised. Further revealed is some irrefutable evidence derived from patient profile analysis and experimental data that seizure detection using sensors positioned on different parts of a patents body ultimately makes an impact on the monitoring of epilepsy, endorsing that modern computer science is providing a timely chance for a more personalised approach to the monitoring and management of epilepsy. 


\section{Table of Contents}

CHAPTER 1 INTRODUCTION

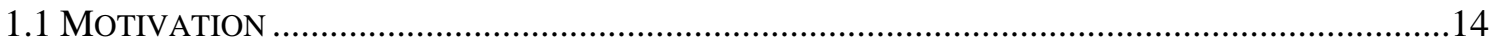

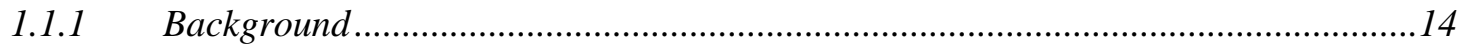

1.1.2 Sensor-Based IoT Device Experiments ................................................................15

1.1.3 Complexity of Epilepsy and Sensor-Based Devices ..............................................17

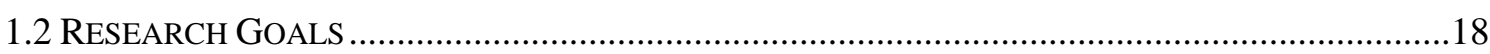

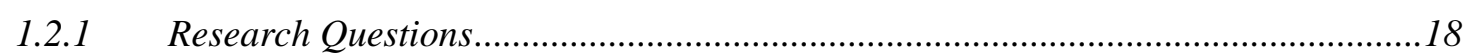

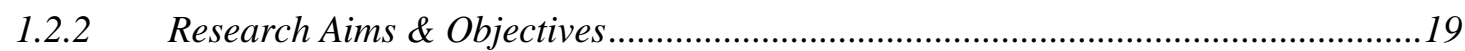

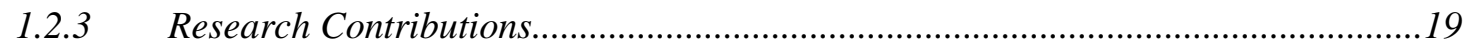

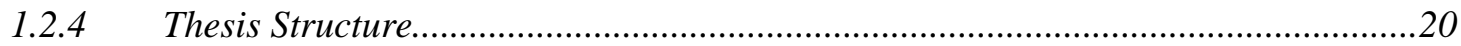

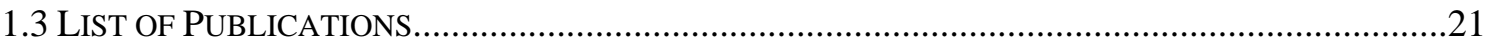

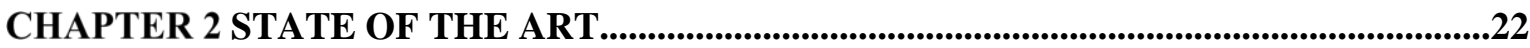

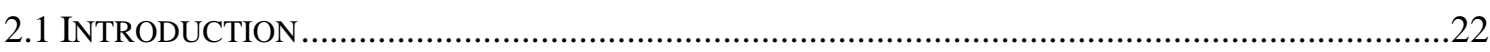

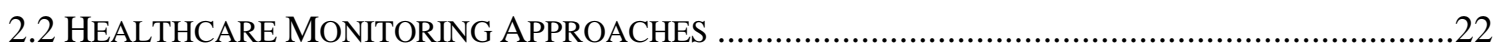

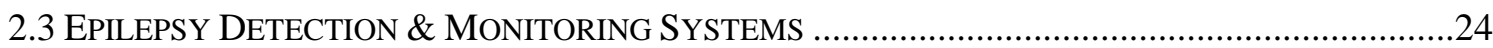

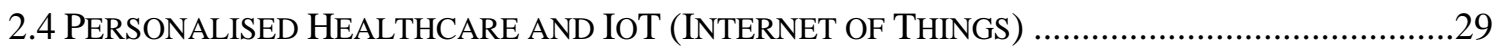

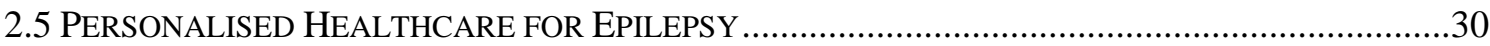

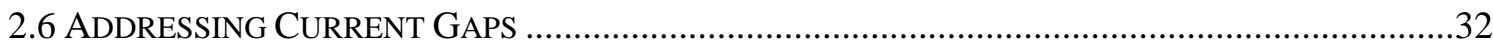

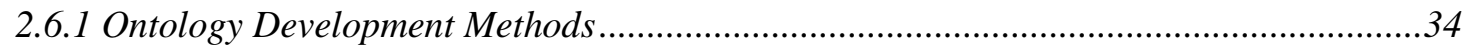

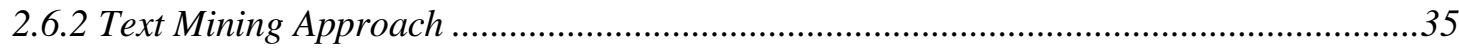

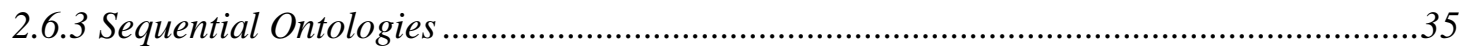

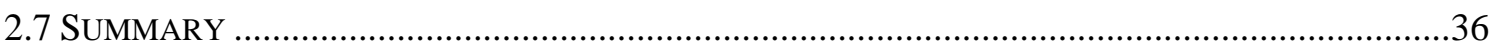

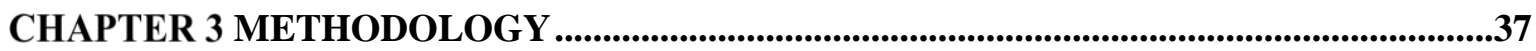

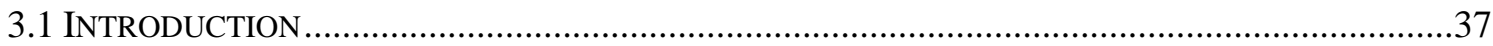

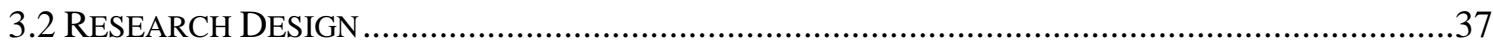

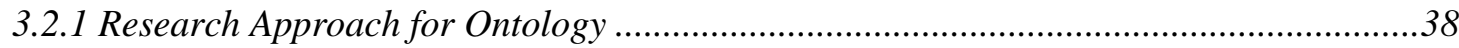

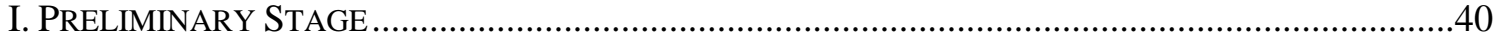

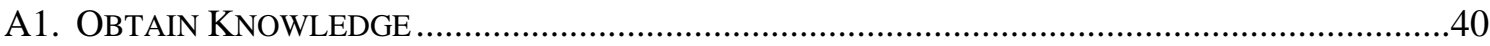

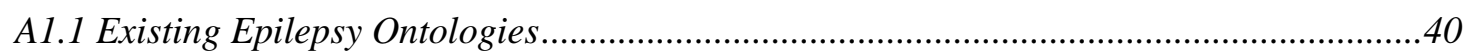

A1.2 The ILAE (International League Against Epilepsy) ………...........................................

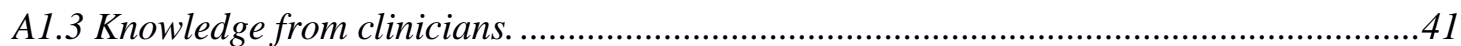

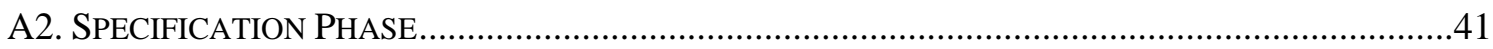

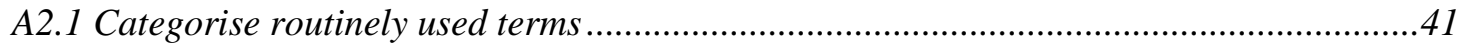

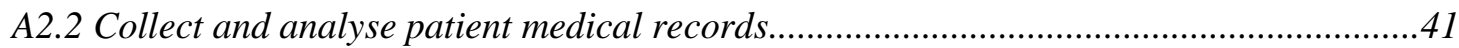

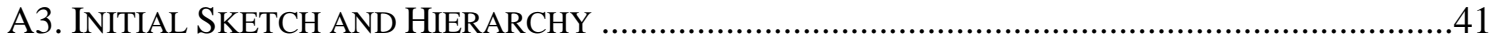


II. ONTOLOGY STAGE.

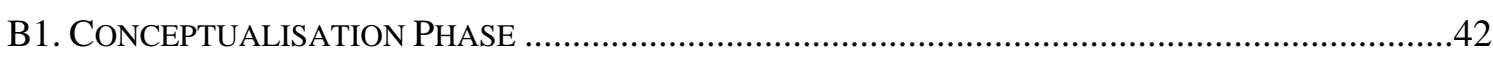

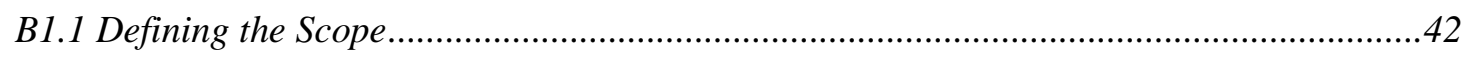

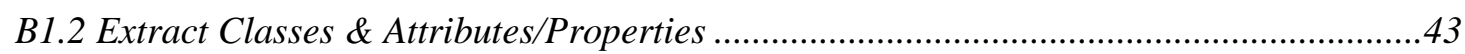

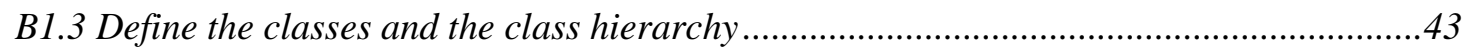

B1.4 Define the 'object properties', 'data properties' and 'individuals' .................................43

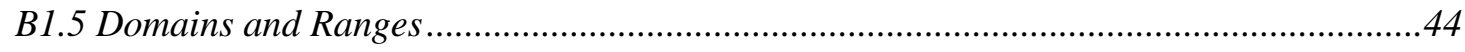

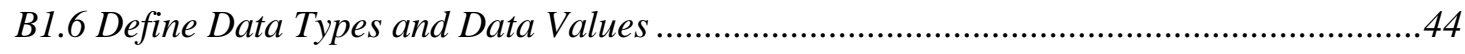

B1.7 Make classes disjoint of each other to avoid multiple inheritance .....................................45

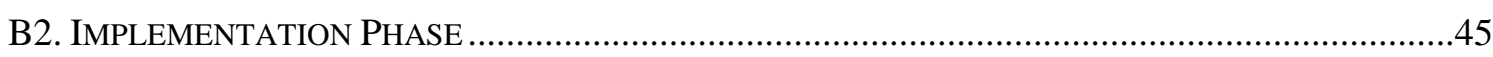

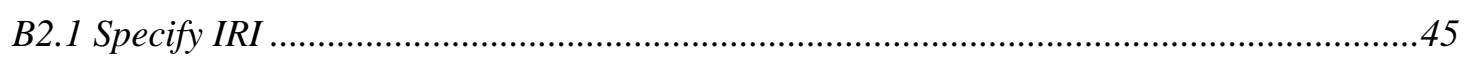

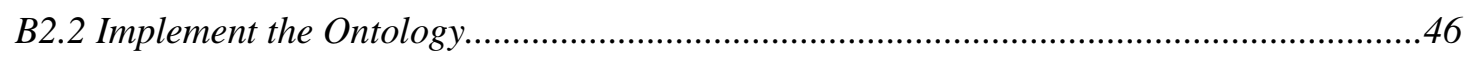

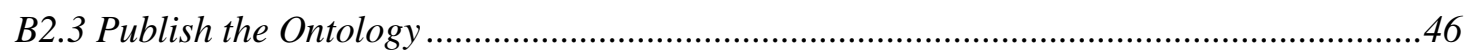

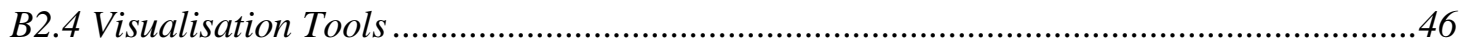

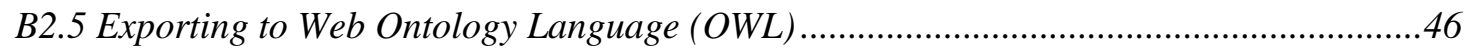

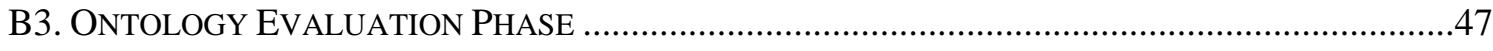

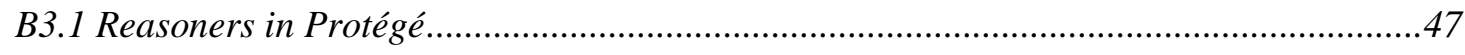

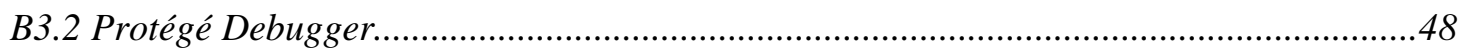

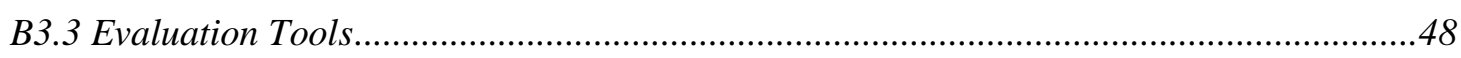

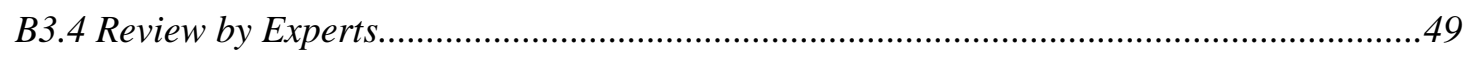

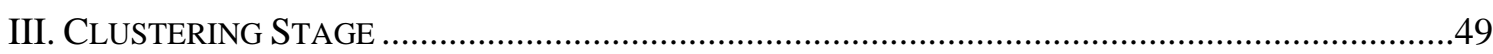

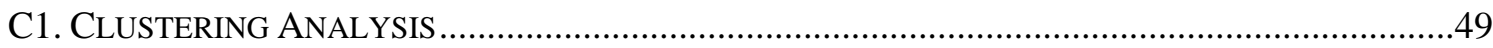

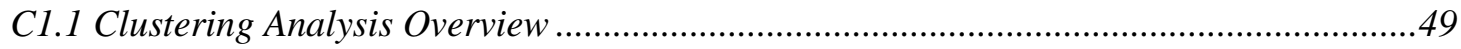

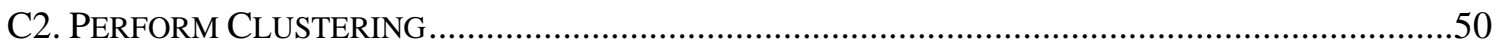

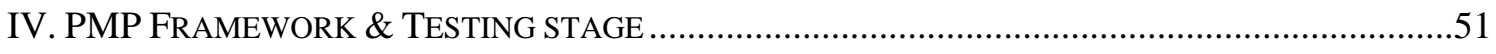

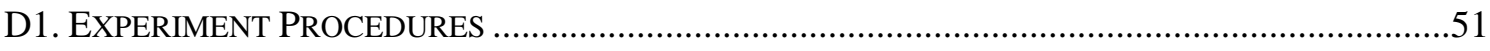

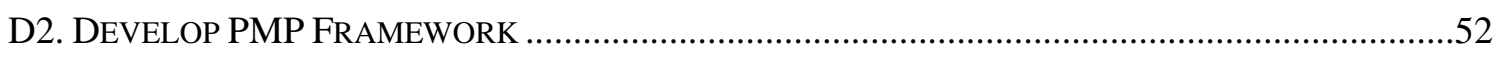

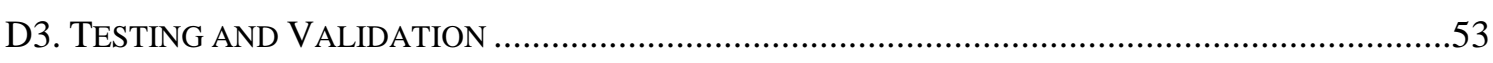

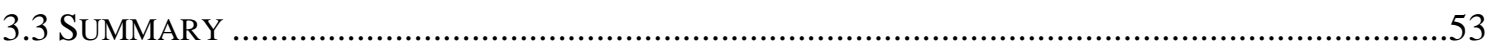

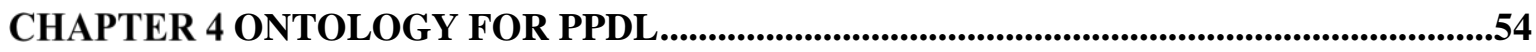

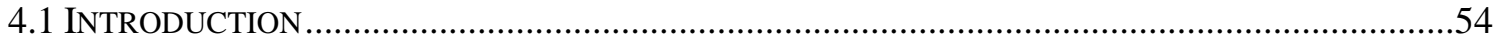

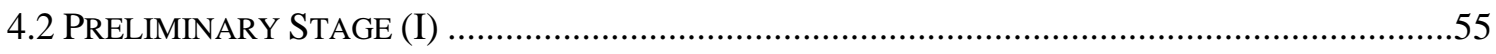

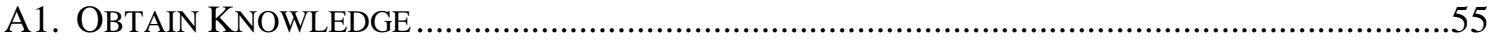

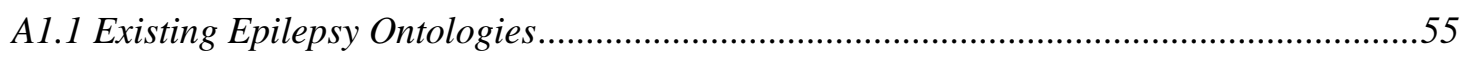

A1.2 The ILAE (International League Against Epilepsy) ......................................................56

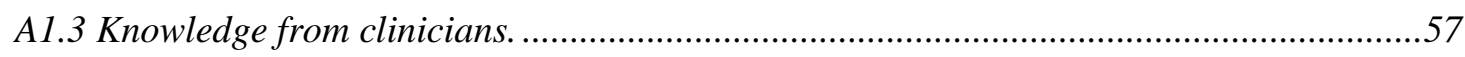

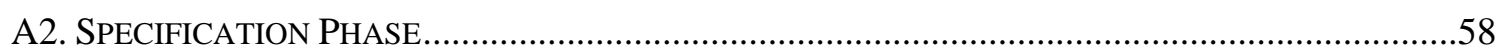

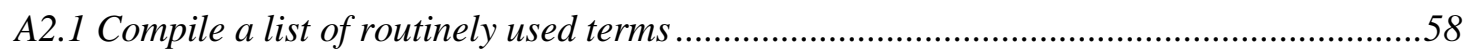

A2.2 Collect and analyse patient medical records.................................................................59 


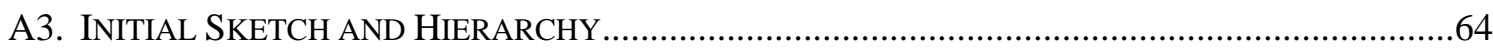

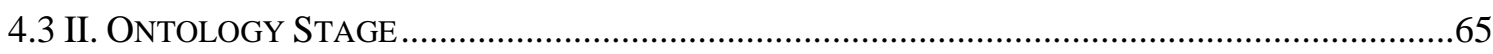

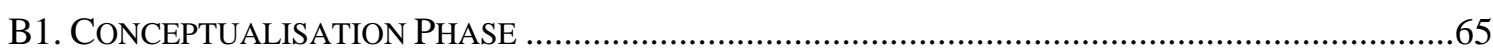

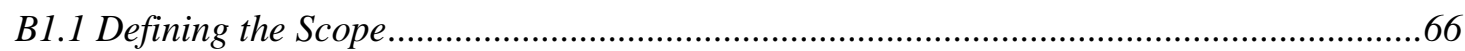

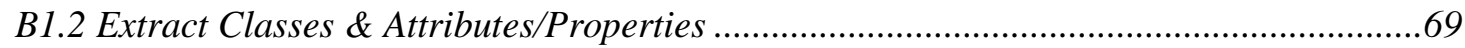

B1.3 Define the classes and the class hierarchy..................................................................... 70

B1.4 Define the 'object properties', 'data properties' and 'individuals' ................................71

B1.5 Domains and Ranges.............................................................................................

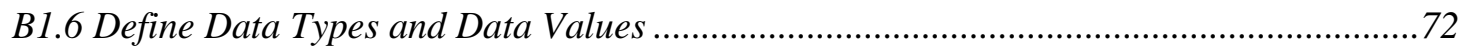

B1.7 Make classes disjoint of each other to avoid multiple inheritance ...................................75

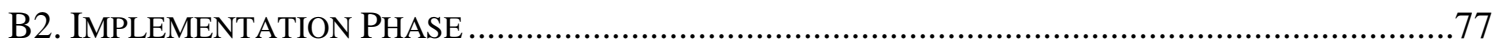

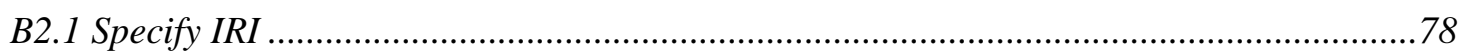

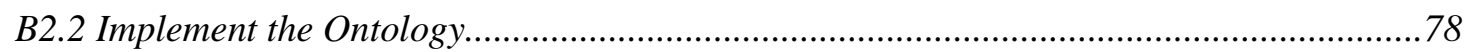

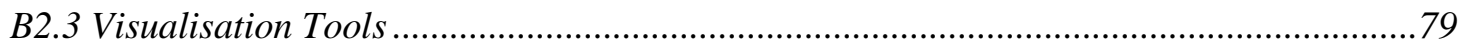

B2.4 Exporting as ESO OWL and XML Serialization for PPDL …..........................................81

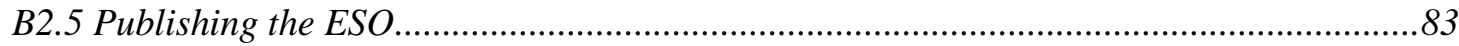

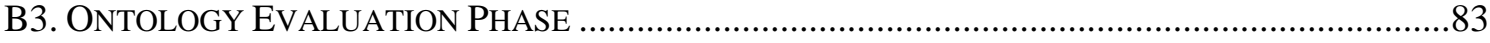

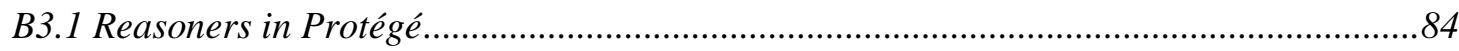

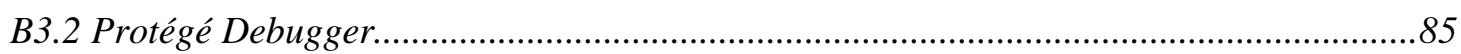

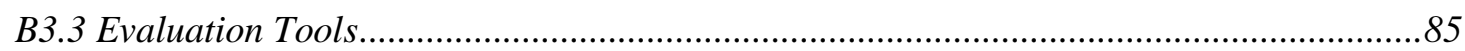

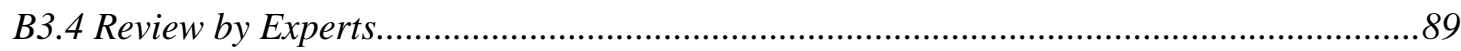

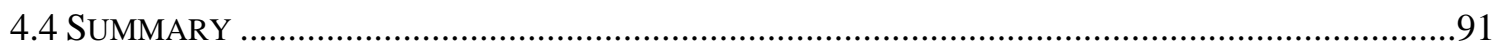

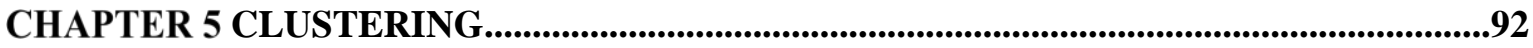

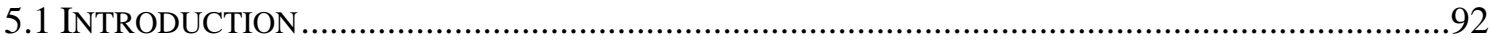

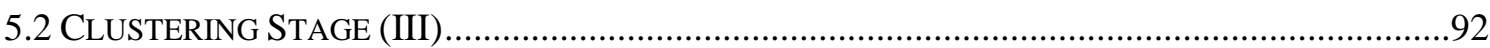

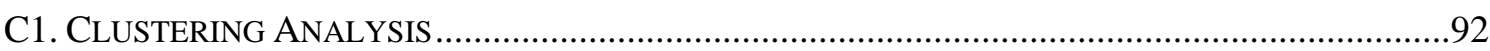

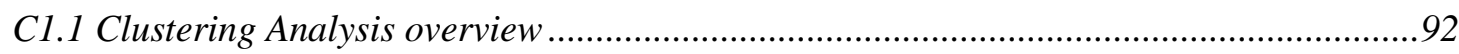

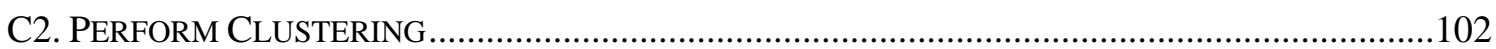

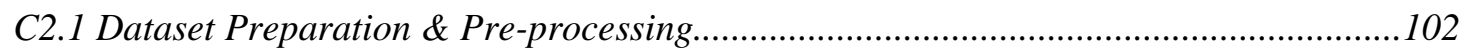

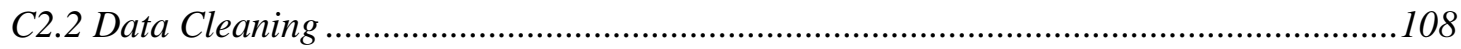

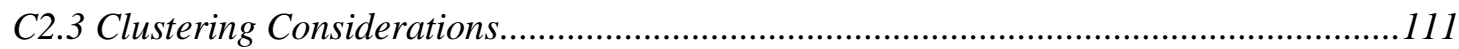

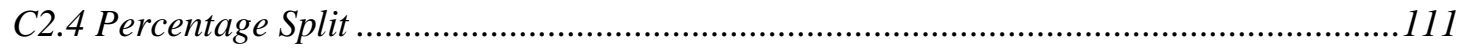

C2.5 Choosing the Seed ..........................................................................................112

C2.6 Choosing the Optimal Number of Clusters ……......................................................112

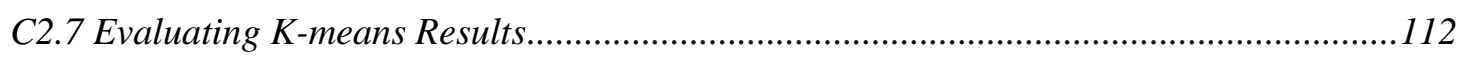

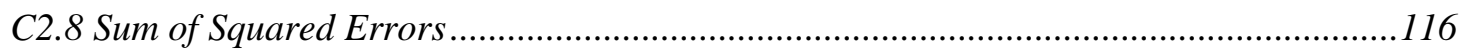

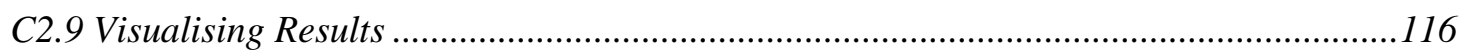

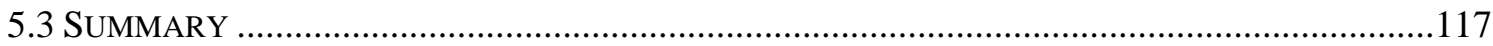

CHAPTER 6 EXPERIMENT, OBSERVATIONS AND FINDINGS ...................................118 
6.1 INTRODUCTION

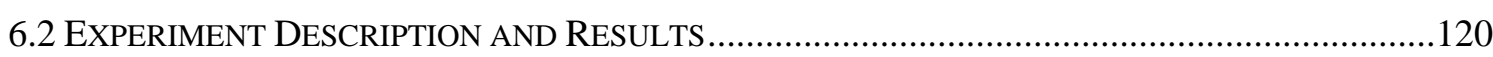

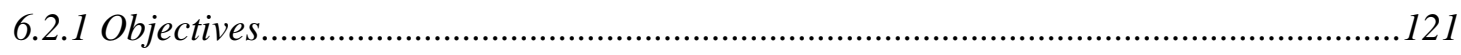

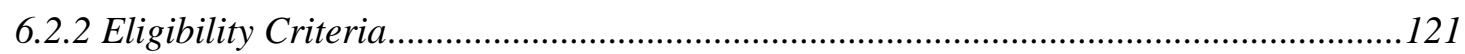

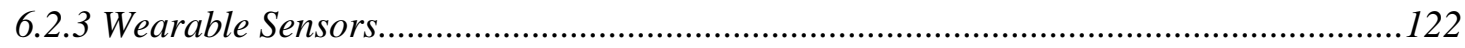

6.2.4 Data Collection \& Analysis ......................................................................................124

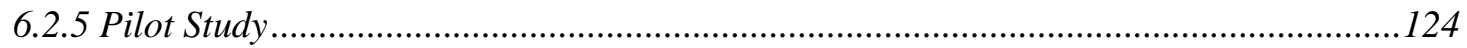

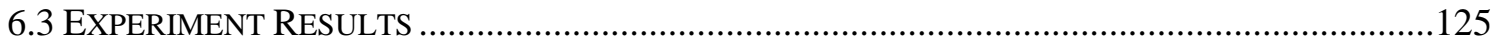

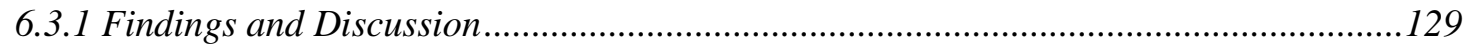

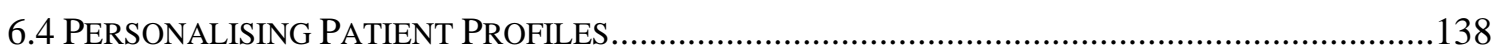

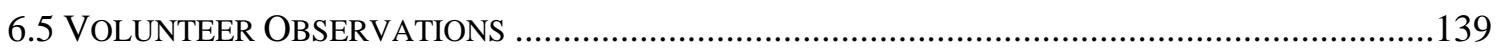

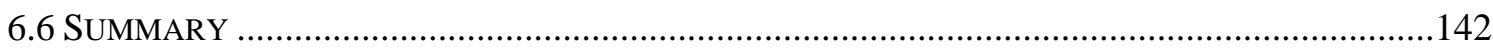

CHAPTER 7 FRAMEWORK FOR PERSONALISED MONITORING PLAN......................144

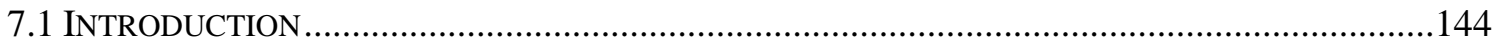

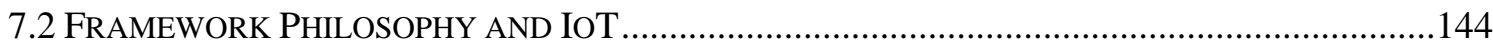

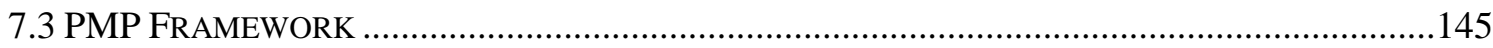

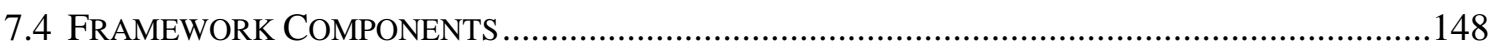

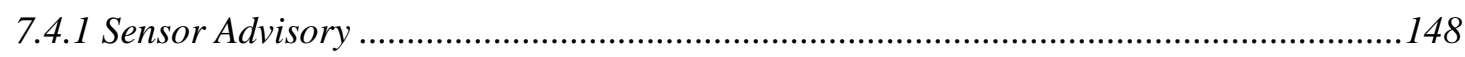

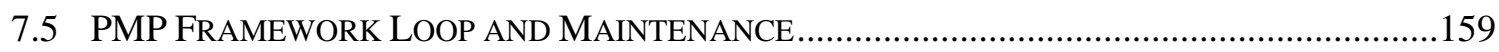

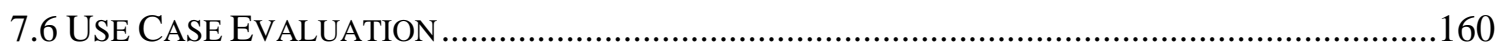

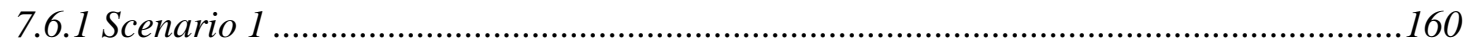

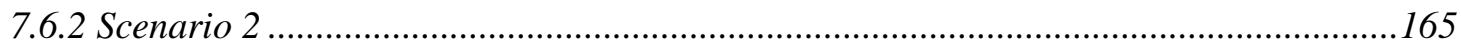

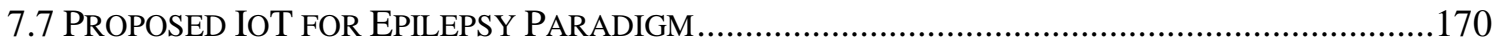

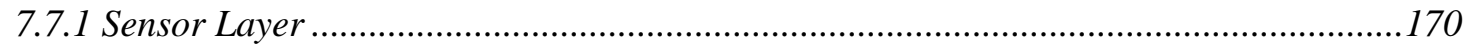

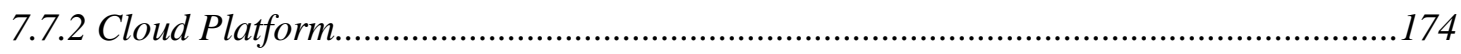

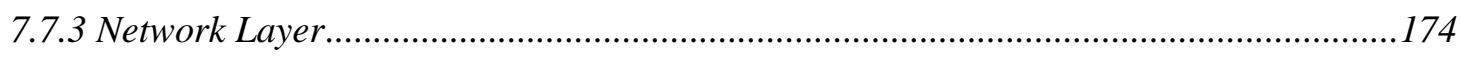

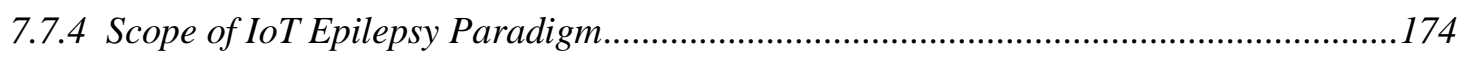

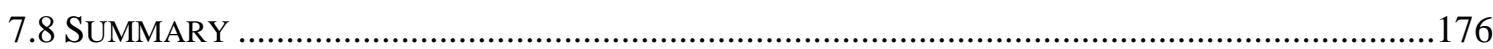

CHAPTER 8 CONCLUSION AND FUTURE WORK..............................................................177

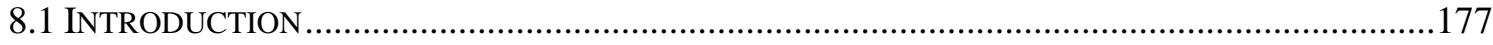

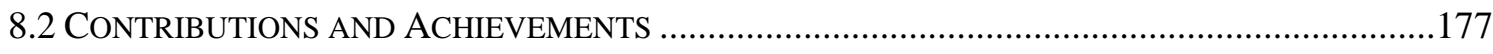

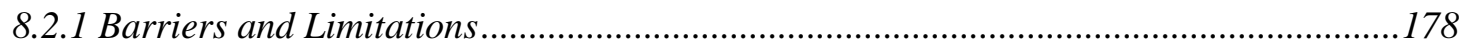

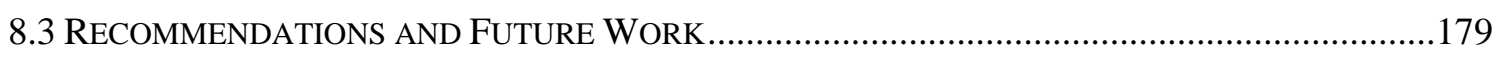

8.3.1 PPDL (Patient Profile Development Language) and Clustering..................................179

8.3.2 Machine Learning and Sensor Decision Tree.............................................................. 179

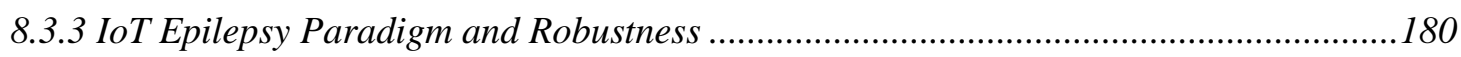

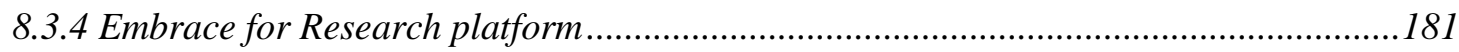

8.3.5 Long Term Uses \& Applicability in other domains for the PMP Framework ................181 


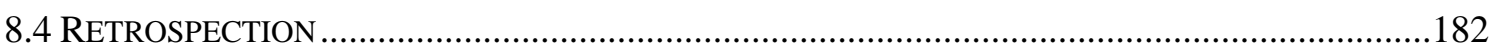

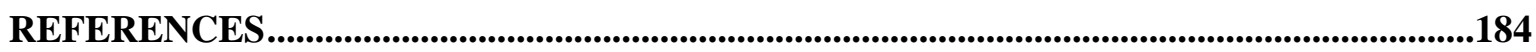

APPENDIX A

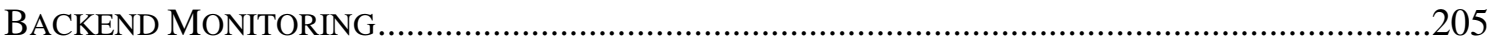

APPENDIX B ........................................................................................................................................206

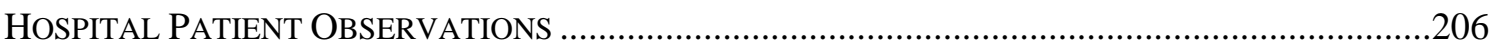

APPENDIX C

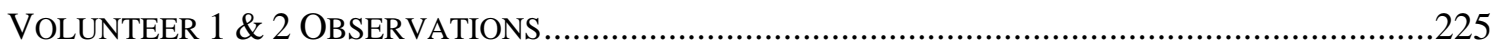




\section{Table of Figures}

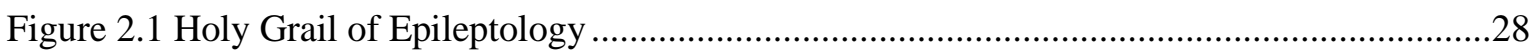

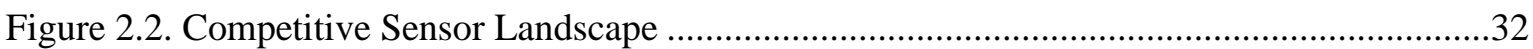

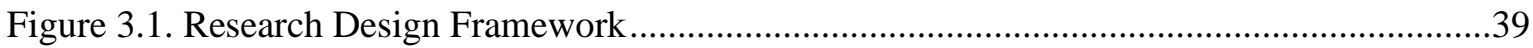

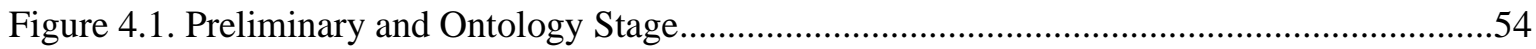

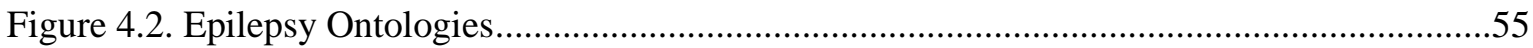

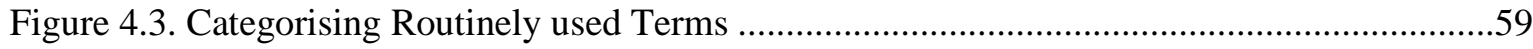

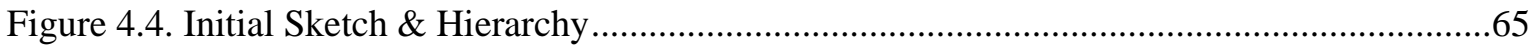

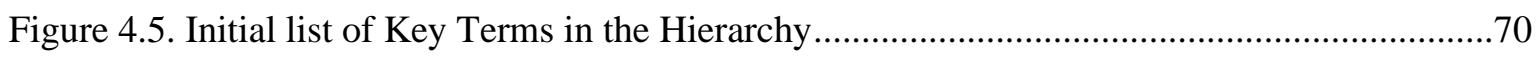

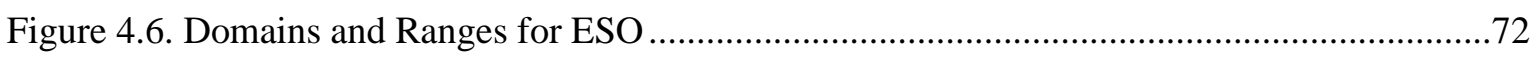

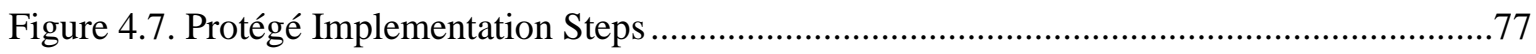

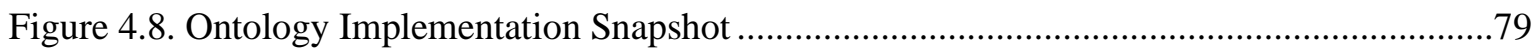

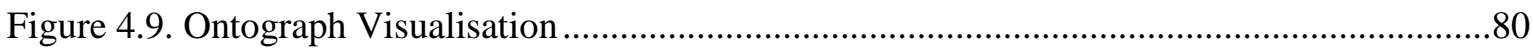

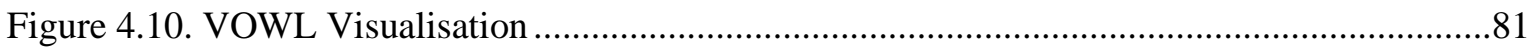

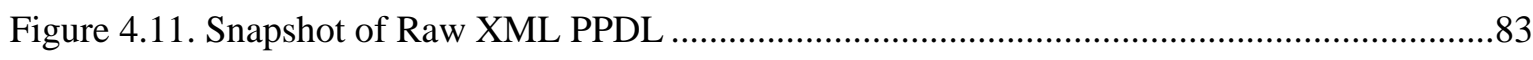

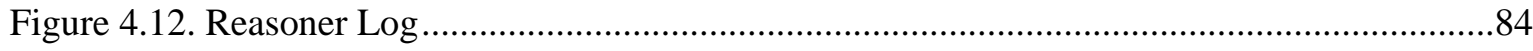

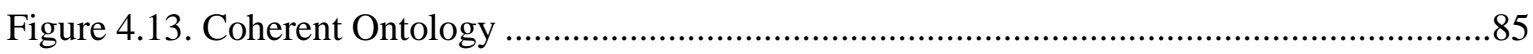

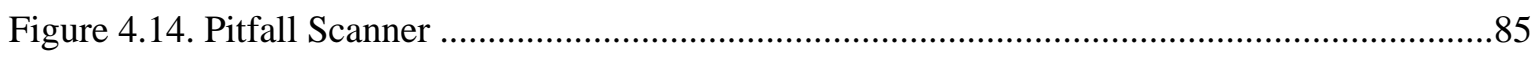

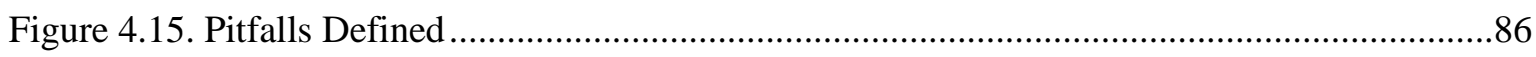

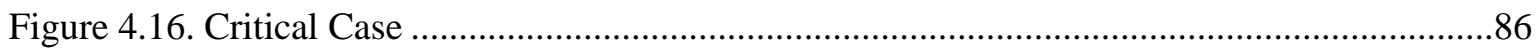

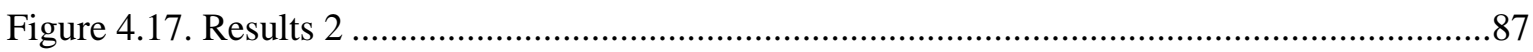

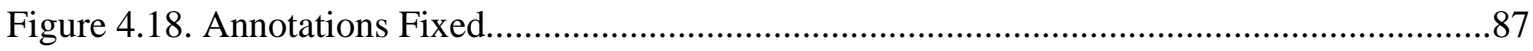

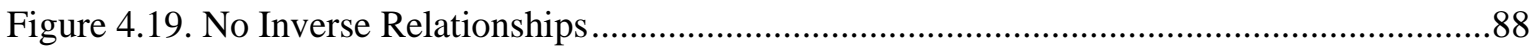

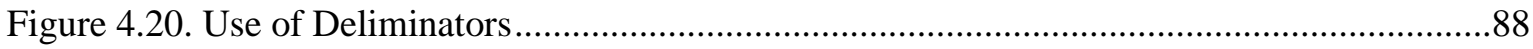

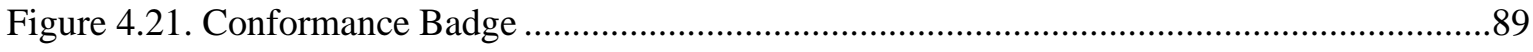

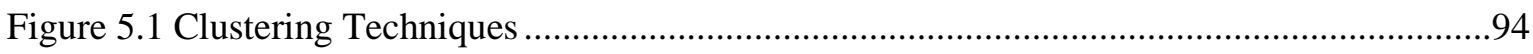

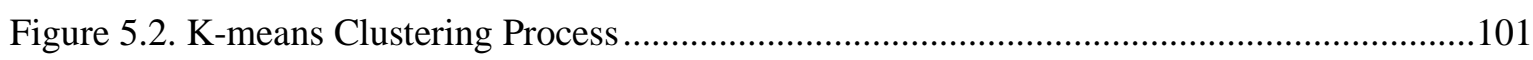

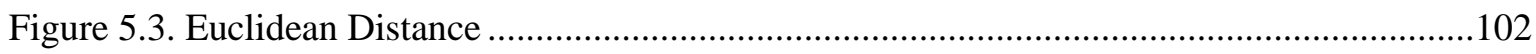

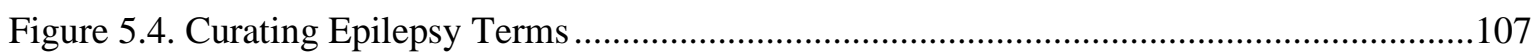

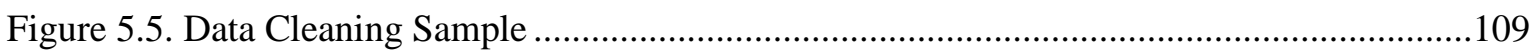

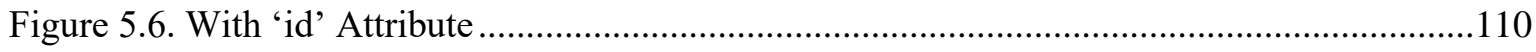

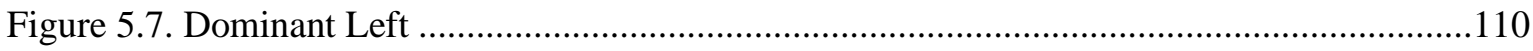

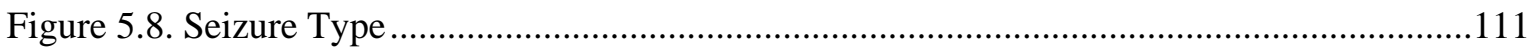

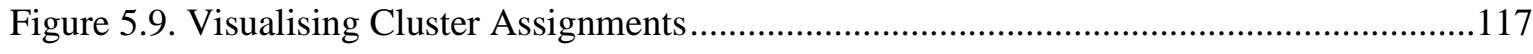

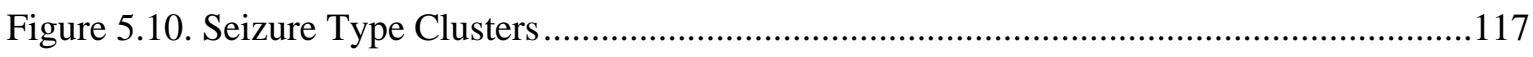

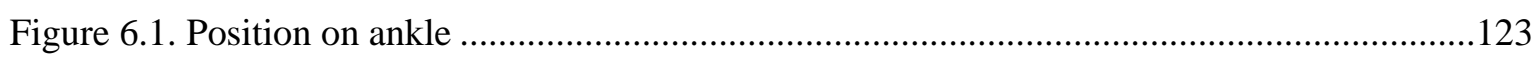

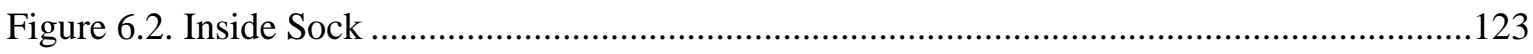

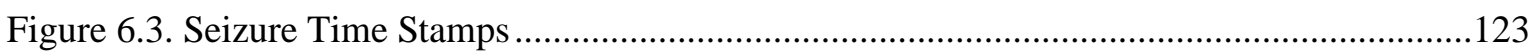

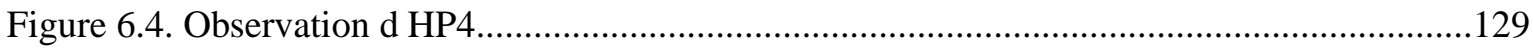


Figure 6.5. Observation b HP4 .130

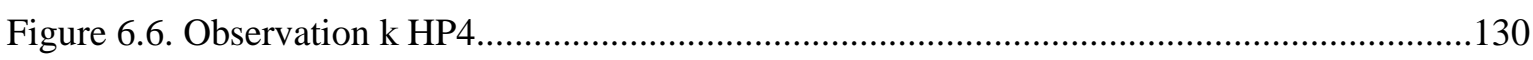

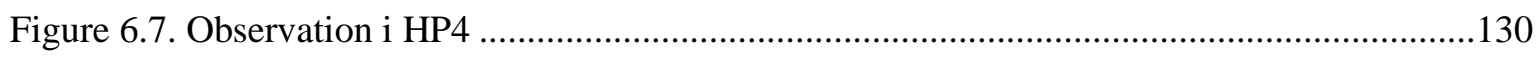

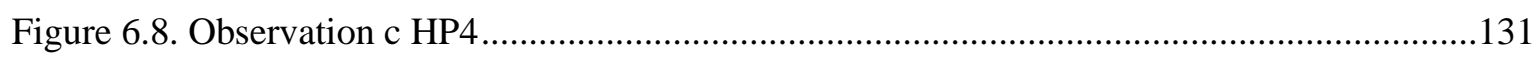

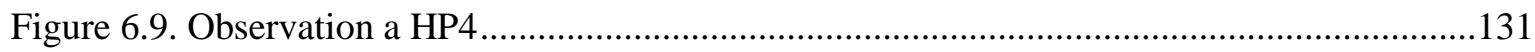

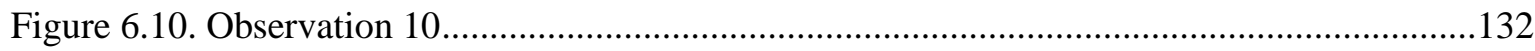

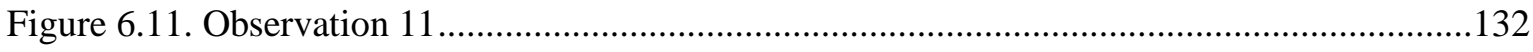

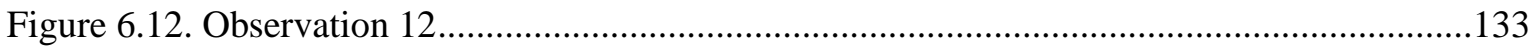

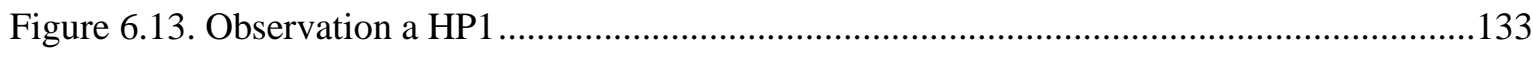

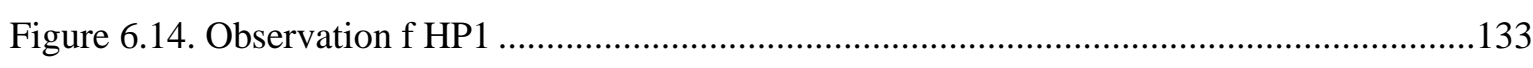

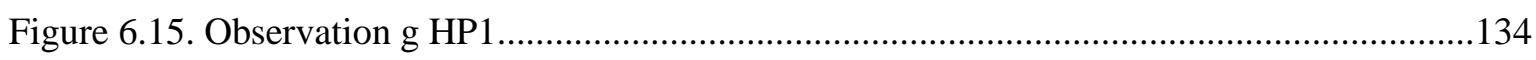

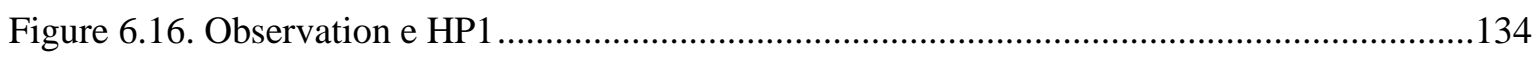

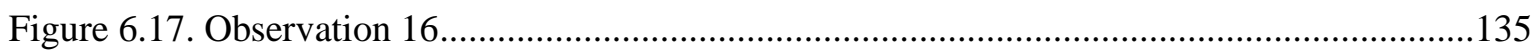

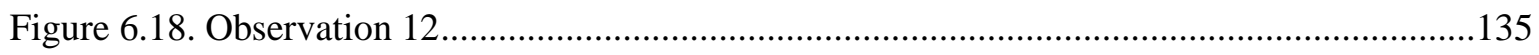

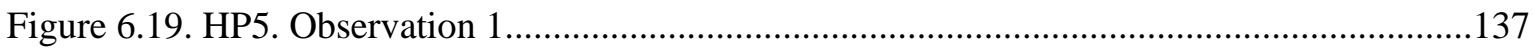

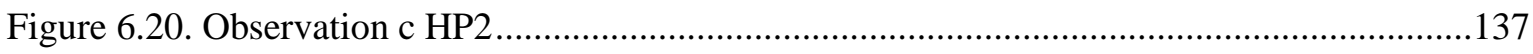

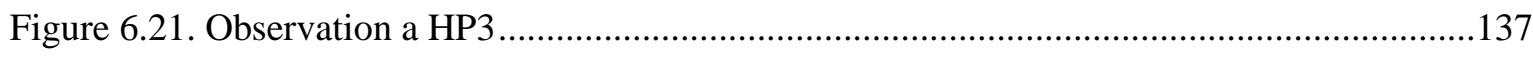

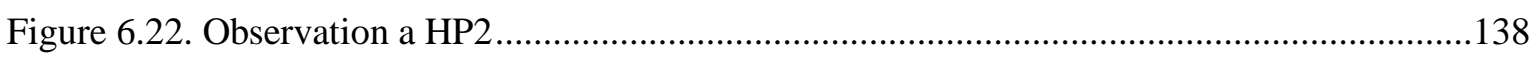

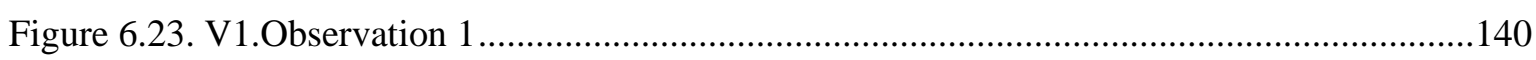

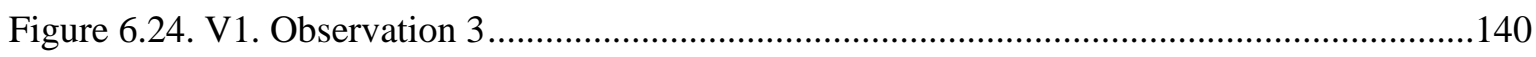

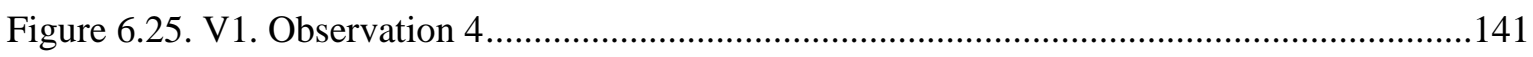

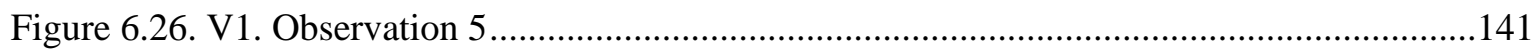

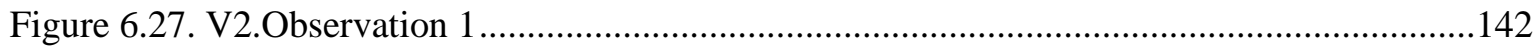

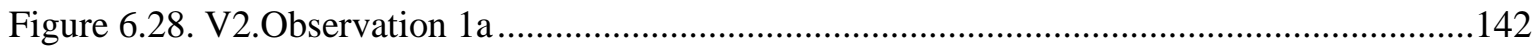

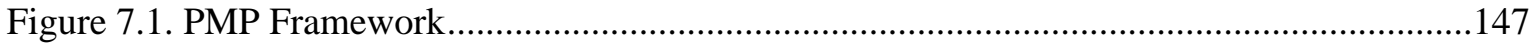

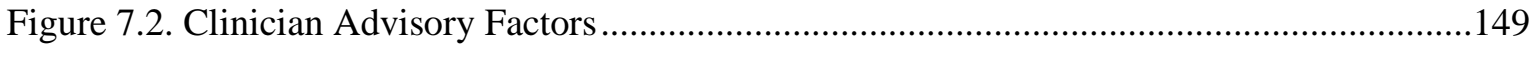

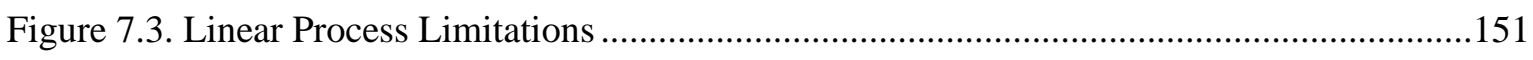

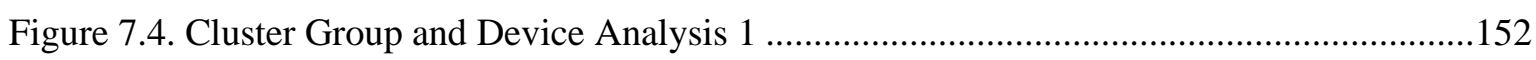

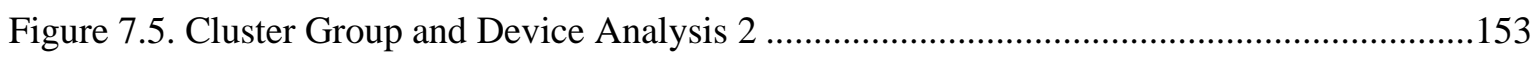

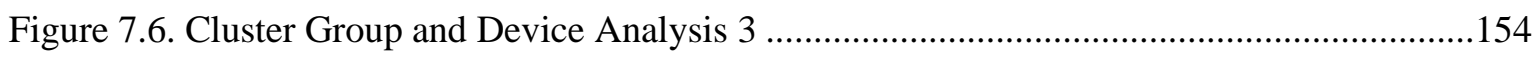

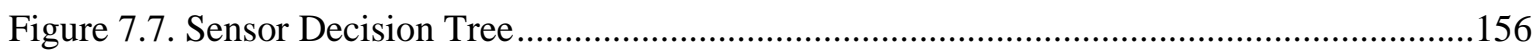

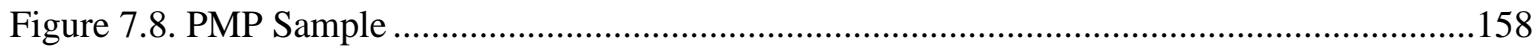

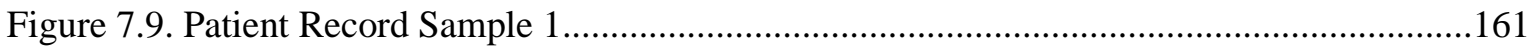

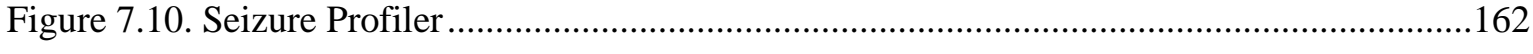

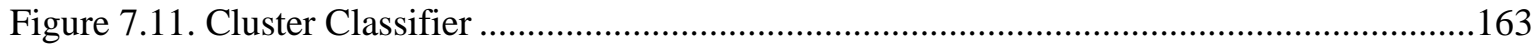

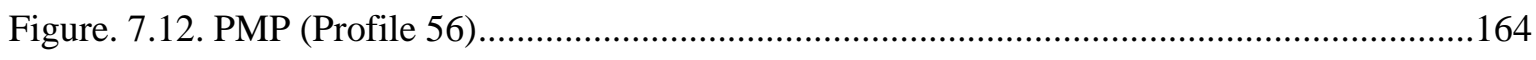

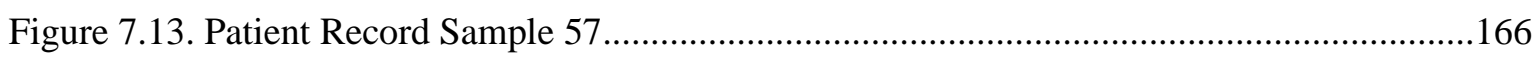

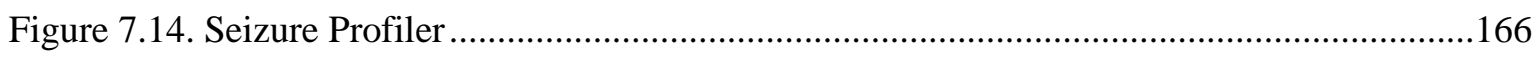

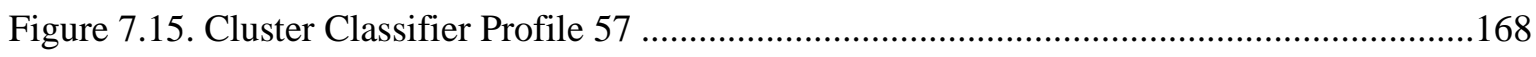

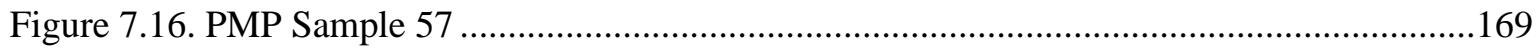




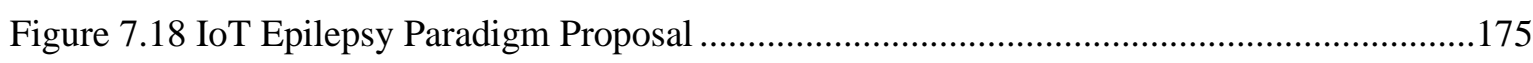

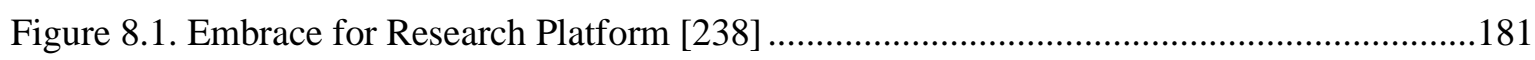

\section{List of Tables}

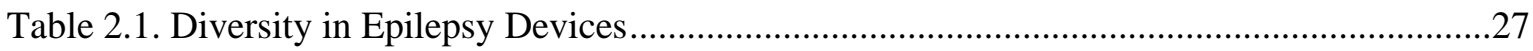

Table 2.2. Smart Approaches to Monitoring in Healthcare and Epilepsy..........................................28

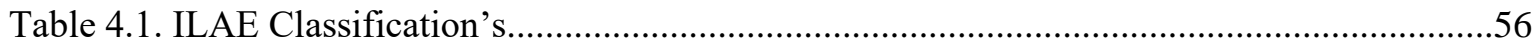

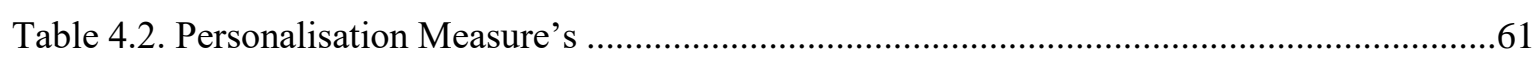

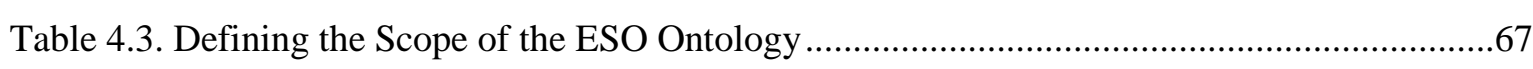

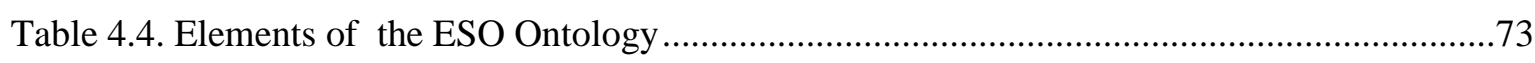

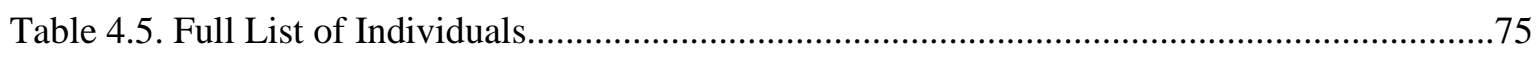

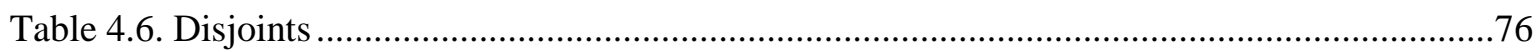

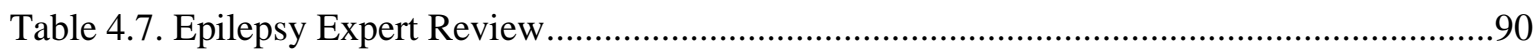

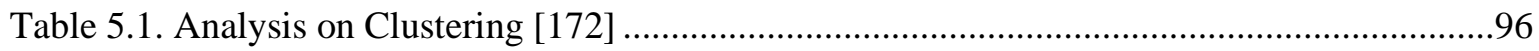

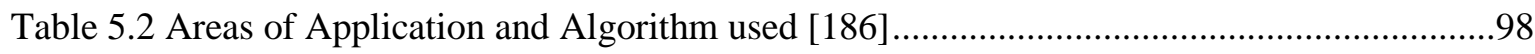

Table 5.3 Parameters to Evaluate Clustering Techniques.................................................................99

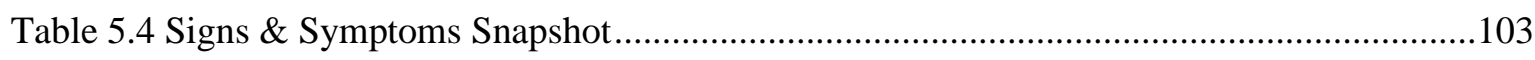

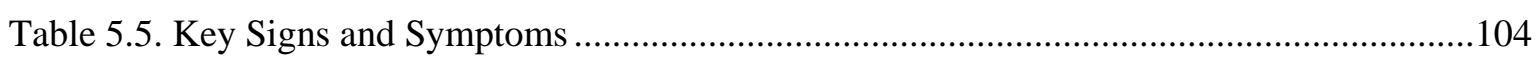

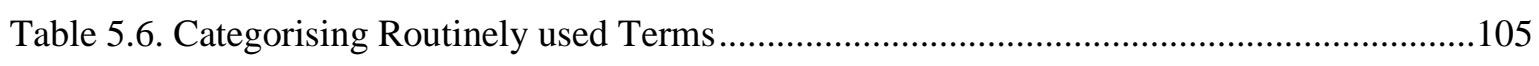

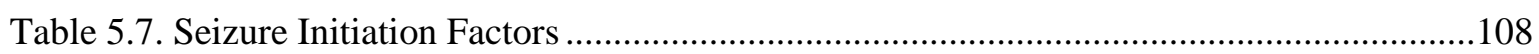

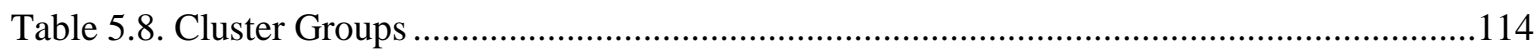

Table 6.1. Analysis of Practicable Devices for Experiment ….........................................................120

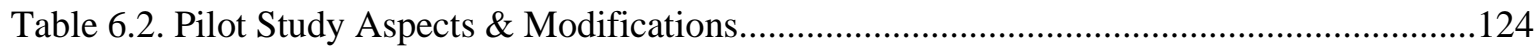

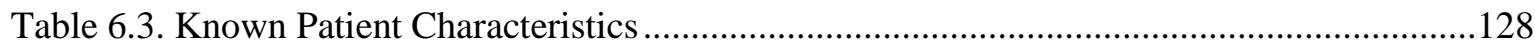

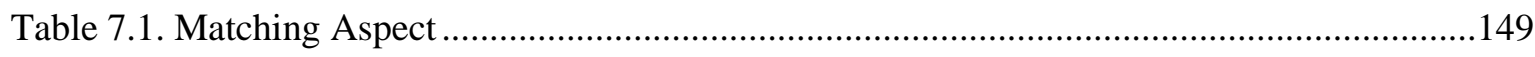

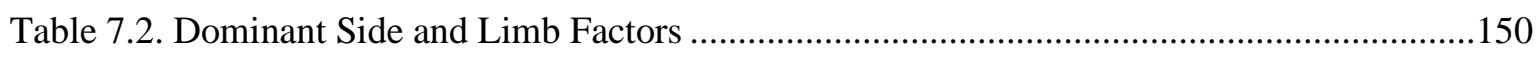

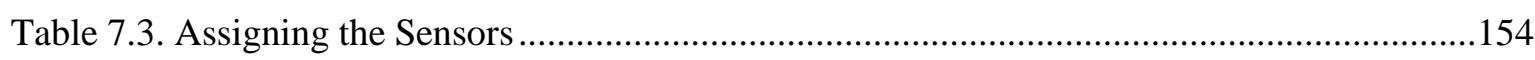

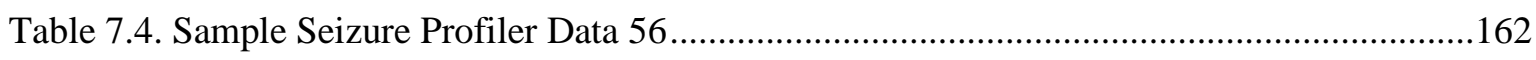

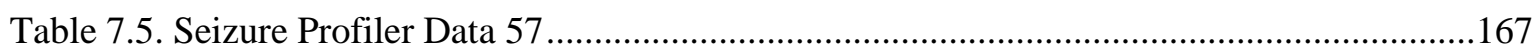

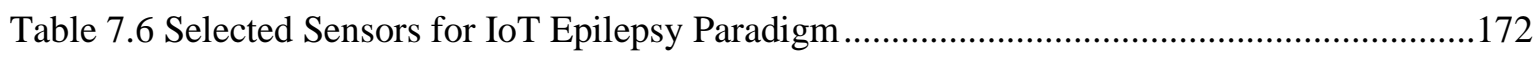

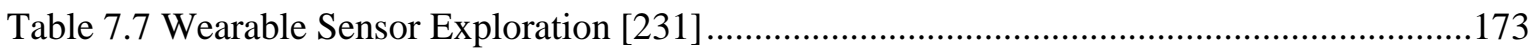




\section{Acronyms}

AACMPE

AI

AIWAC

APP

CEL

CMTHC

CRM

CSS

DAML

DKAP

DL

EDC

EEG

EM

ESO

GSR

GT

GUI

$\mathrm{HCP}$

HR

HTML

HTTP

ILAE

IoT

IRAS

IRI

JSON

KMS

LD

$\mathrm{MCC}$

NCBO

NHS
Allergy and Asthma Care in the Mobile Phone Era

Artificial Intelligence

Affective Interaction through

Wearable Computing

Application

Classifier for [equation]

Children's Medical Treatment and Healthcare System

Customer Relationship Marketing

Cascading Style Sheet

DARPA Agent Mark-up Language

Domain Knowledge Acquisition Process:

Description Logic

Euclidean Distance Calculation

Electroencephalogram

Effects Monitor

Epilepsy Seizure Ontology

Galvanic Skin Response

Glossary of Terms

Graphical User Interface

Health Care Professional

Heart-Rate

Hypertext Mark-up Language

Hypertext Transfer Protocol

International League Against Epilepsy

Internet of Things

Integrated Research Application System

Internationalized Resource Identifier

JavaScript Object Notation

Knowledge Management System

Linked Data

Mobile Cloud Computing

National Center for Biomedical Ontologies

National Health Service 
OIL

OOPS

OWL

PDF

PHS

PMP

PPDL

RACER

RDF

ROCHAS

Sami

SMS

SSE

Trowl

UI

URI

VNS

VOWL

WEKA

WHS

XML
An Ontology Infrastructure for the

Semantic Web

Ontology Pitfall Scanner

Web Ontology Language

Portable Document Format

Personalised Healthcare Systems

Personalised Monitoring Plan

Patient Profile Description Language

Renamed abox and Concept

Expression Reasoner

Resource Description Framework

Robotics and Cloud-assisted Healthcare System for Empty Nester

Sleep Activity Monitor

Short Message Service

Sum of Squared Errors

Tractable Reasoning infrastructure for OWL 2

User Interface

Uniform Resource Indicator

Vagus Nerve Stimulation

Visual Notation for OWL Ontologies

Waikato Environment for Knowledge Analysis

Wireless Health Sensors

Extensible Mark-up Language 


\section{Chapter 1 Introduction}

\subsection{Motivation}

A number of epilepsy and healthcare monitoring systems have been proposed by researchers to detect seizures and monitor the patient. The majority of these methods of management focus solely on how we can improve 'seizure detection and prediction'. For example, epilepsy specific platforms include and Sareen and EpiCare. Sareen gathers data from EEG, (electroencephalogram, which records brain activity) using Mobile Cloud Computing (MCC) for storage and for sending notifications to the family and clinicians [1]. Whereas EpiCare, whilst also using MCC is used in home settings specifically to assist epilepsy diagnosis [2] and portable EEG are used to detect the seizures.

Other body measurements in addition to EEG have been recommended i.e. signals obtained from sensors have been proposed in many academic studies and commercial sectors to detect epileptic seizures. For example, gyroscopes, accelerometers, pulse rate, temperature sensors, magnetometers, galvanic skin response sensors (GSR), implanted advisory system, electromyography, video detection systems, mattress sensor, and audio systems [3]. Body sensor networks placed on the body together with MCC has been proposed for epilepsy detection [1] such as pulse rate which can be worn as a wrist band.

Yet none of these approaches to manage epilepsy recognises that each epilepsy patient is unique in their specific characteristics of epilepsy. The fact is that epilepsy is complex and seizures are a not easily managed due to being a very diverse group of disorders, [4] just because someone has epilepsy it does not tell you the nature of their epilepsy or what seizure behaviours they have, seizures can be different for each person [5]. A question that needs addressing is whether epilepsy monitoring can benefit from personalised approach.

\subsubsection{Background}

Epilepsy is a chronic neurological disorder characterized by recurrent and unprovoked seizures. The epileptic seizures occur from electrical instabilities in the brain which can range from brief lapses of attention or muscle jerks to severe and prolonged convulsions [6].

The patient's quality of life is dramatically impacted and there are about 65 million people affected by this disorder worldwide.

Epileptic seizure monitoring and management is challenging. Most current studies of epileptic seizure detection disclose drug resistant epilepsy still lacks an ultimate solution, despite the increase in anti-epileptic drugs [7]. 
Epilepsy is not a single disease, but a family of syndromes that share the feature of recurring seizures. In some instances, it may be related to a genetic aetiology, or it can occur in association with metabolic disorders, structural abnormalities, infection or brain injury [8].

In the United Kingdom epilepsy affects 3 million people and in the United States it is the 4th most common neurologic disorder, only migraine, stroke, and Alzheimer's disease occurs more frequently [9]. There are around 60 different types of seizure and a person may have more than one type. Seizures vary depending on where in the brain they are happening. Some people remain aware throughout, while others can lose consciousness [10].

Aside from their unpredictability, the worst part of having seizures is their utter complexity. The complex nature of epilepsy is noticeable in the variation of seizures types and symptoms between one patient and another. Distinguishing or classifying an individual epilepsy patient makes it difficult to manage and monitor. The negative impact of uncontrolled seizures spreads beyond the individual to affect their family, friends, and society. Chronic anxiety is experienced by the families and friends of people with epilepsy and many lives are adjusted to ensure the safety of their loved one. Novel approaches to epilepsy treatment are still greatly needed [11] novel therapies that better manage and monitor seizures as well as technology can help to handle the consequences of seizures.

Insufficient knowledge about epilepsy, which is a very common disorder, has a great and negative impact on people with epilepsy, their families and communities, and the healthcare systems. There is need for a better understanding of the disease to make way for new approaches to monitor it.

In the modern day of personalised medicine and rapid advancements in IoT a question that needs addressing is whether epilepsy monitoring can benefit from personalised approach. Can the IoT have the potential to significantly improve the 'patients' daily lives whose seizures cannot be controlled by either drugs or surgery? [12]

\subsubsection{Sensor-Based IoT Device Experiments}

One of the challenges in using sensor-based IoT devices to achieve a personalised approach is the barriers found in the use of them in hospital settings. EEG monitoring is the chief gold standard method used within hospitals to detect and monitor seizures. EEG is an electroencephalogram is a recording of brain activity. There is limited evidence found how sensor-based IoT devices and experiments are used in hospital settings.

Recently an epilepsy patient described what a more connected system might have been able to do, in an article; 'Where is the internet of healthcare things?' and stated; "I could have a different result if I (my device) was connected to the correct APP/Web Portal” [13]. Generic smart devices are being recommended to patients without any precision. Even though there is 
much hype about new healthcare sensor based IoT devices in today's digital world; many clinicians presume they should be up to date with latest wearable technology or should be recommending them to their patients, but they don't have this knowledge in fact it is quite uncommon to find a doctor so well versed and confident in technology [14]. Yet the impact of technology on 'future' healthcare is mammoth and if these changes hit healthcare professionals unprepared; "they will wash away the medical system we know and leave it a purely technology-based service without personal interaction. [14].

Here the 'personal' element of technology is identified; it is with this aspect in mind that the next logical step in digitalising healthcare must be embraced. This next step coming around is a more 'personalised' approach to healthcare and current research is identifying this as a new trend in medicine focusing on patient-centered care [15].

There is evidence that sensor-based IoT devices prescribed for individual patients are not yet fully fit for purpose. Once such study looked at some significant unintended consequences relating to new security vulnerabilities and modifications of behaviour together with use of big data sets and unanticipated challenges faced by regulatory authorities thus making it difficult to keep pace with recent innovations [16]. Currently there are epilepsy sensors and apps that track medication, send reminders to take medication, some are for emergency with buttons to press before losing consciousness with a seizure and some detect a seizure based on the pattern of jerks [17]. However, there is a danger that epilepsy patients and their Doctors who are confronted with a bewildering range of devices [18] may have difficulty in coming to a sound understanding of the device.

Therefore, it is vital to choose the most appropriate sensor, to find evidence on device reliability for a particular seizure type, and specifically to avoid any false alarms which can be troublesome for carers or family. It is beneficial for epilepsy patients where possible to discuss with their doctors the advantages and disadvantages of each device [19]. Tailoring the approach to patient sensors is noteworthy. The main factor of a devices value may ultimately be its ability to provide meaningful, accurate, and timely information [20] and as observed: [21] address the vital purpose of improving patient outcomes by providing guidance to the user. Therefore, preparations need to be in place to realise these goals. There is still time and measures that can be taken to 'guide' clinicians to choose the most precise device.

Sensor-based IoT devices are in popular demand for healthcare providers since there is a constant need for patient monitoring, this drives the sensor usage, therefore patients need to be monitored pre-hospital, in-hospital (before, during, and after procedures), at home, in longterm care, and in other locations [22] yet it is argued that there is limited information about the actual patient care-related tasks that physicians perform on these devices [23]. Furthermore, time is vital for hospital-based physicians to have the ability to obtain the right information at the right place [23]. Hence medical devices and apps are already proving invaluable tools for healthcare professionals, but as their features and uses expand, there is a 
danger that because they are becoming even more widely incorporated into nearly every aspect of clinical practice [24] there may not be the knowledge or tools to match the correct device with the correct patient.

There are very few experiments with sensor-based IoT devices that have been endorsed by the NHS and a large problem is poor information when caring for people with epilepsy or doing epilepsy clinical trials [25] yet there is great potential to vastly increase the efficacy of epilepsy management using biomedical devices that can improve the quality of information. As available devices and sensors grow, if clinicians could be provided with more guidance in understanding and choosing which sensor suits which situation then a personalised approach can be achieved and hence the management and monitoring of epilepsy can be improved. Even now seizure detection devices are at a relatively early stage of development and so far it is difficult to find large-scale studies that compare the effectiveness of one device against another [26]. This highlights that more experimental evidence is needed.

\subsubsection{Complexity of Epilepsy and Sensor-Based Devices}

With so many existing sensor-based IoT devices for epilepsy devices, together with associated factors and with so many eventualities in capturing a seizure at home, it is difficult to know the best device to implement. For example, the patient could be in the bath, in bed, or watching the TV when the seizure occurs and it could be that a Smart Watch used for monitoring shaking may not be comfortable to wear in bed, so a Smart Mattress would take its place. Likewise, a Companion Monitor which monitors bed movement and sound but would not be fitting in the shower [27]. Another variant is that different types of seizures will present themselves and there aren't always the solutions at the clinician's fingertips; devices are just not suitable for all the different environments we live in.

It is so difficult to ultimately 'monitor' epilepsy because of these complexities. A demand for a more personalised approach to monitor the 'individual' patients' characteristics is needed. This personalised approach is addressed in this thesis with the creation of the PPDL. Here the 'terminology' of epilepsy is investigated so that an 'individual' seizure type patient profile can be formed. To enable the health carers to define a 'type' of epilepsy patient, this thesis also recommends distinct groups of epilepsy patients that share similar characteristics using Clustering Analysis. 


\subsection{Research Goals}

The principal aim of this thesis is to investigate whether a personalised approach to the remote monitoring and management of epilepsy is achievable. Initial research in the area of epilepsy and healthcare monitoring systems has identified the complex facets of epilepsy, also the use of IoT sensor-based devices has identified that experimental evidence is limited.

However, little focus has been placed on how a personalised (individualised) patient-specific approach to managing epilepsy could be feasible to managing epilepsy. This is investigated in this thesis.

This research attempts to address the remote personalised monitoring of individual patients using an IoT approach, highlight the essential IoT sensor-based devices that can monitor epilepsy and show how the typical seizure patient profiles be represented.

Most of the challenges in seizure management lie in its complex nature. This thesis focuses on utilising computing in particular IoT technology to investigate, unravel and decipher some of its many facets.

To begin this journey and realise if a personalised approach can benefit, epilepsy 'terminology' was first investigated; existing seizure type classifications must be understood so that an individual seizure type patient profile can be formed and thus an ontology developed to model this concept.

In addition to the individual patient profile another approach in this thesis was to see if distinct group of patients sharing similar characteristics could be identified so that ultimately patients could receive distinct recommendations per group. In order to achieve this Clustering techniques were analysed and carried out.

Finally, by use of experiments the assumption that an individual patient profile or distinct group of patients sharing similar characteristics can make a difference in which IoT device to use will be evaluated. For example, can an IoT sensor-based device with an accelerometer sensor worn on the left ankle detect a seizure on 'Patient Profile 1'? Or an IoT sensor-based device worn on the right wrist detect a seizure on 'Patient Profile 5'?

\subsubsection{Research Questions}

The main questions this thesis has attempted to answer are outlined below.

RQ 1. How can remote monitoring of individual patients be personalised using an IoT approach?

RQ 1a. Can IoT sensor-based devices support the essential sensors in the reading of bio-signals for epilepsy monitoring? 
RQ 1b. How can an understanding of typical seizure patient profiles be achieved?

\subsubsection{Research Aims \& Objectives}

Following the related concepts previously introduced in this chapter the research aims and objectives for this thesis are outlined below:

Aim: The aim of this research is to develop an IoT driven Framework for remote personalised monitoring and managing of epilepsy

OB1: Investigate ontologies for seizure types/classifications

OB2: Develop a language PPDL (Patient Profile Description Language) using a Semantic Ontology

OB3: Perform a clustering technique to discover distinct groups of patients that share similar characteristics.

OB4: Investigate IoT sensor-based devices that can support the essential sensors in the reading of bio-signals for epilepsy monitoring.

OB5: Design and develop a conceptual framework which will support a Personalised Monitoring Plan (PMP).

OB6: Test and validate the framework with epilepsy scenarios.

\subsubsection{Research Contributions}

The main contributions this research makes are:

- New ontology language; Patient Profile Description Language (PPDL). This language will support the need of the healthcare process to transmit, re-use and share individual patient profile data related to their seizures.

This has been achieved by the investigation of epilepsy 'terminology'; existing seizure type classifications/categories were analysed so that an 'individual' seizure type patient profile could be formed. A close collaboration with clinicians helped to build a data model fit for real-world adoption inside hospital settings and thus an ontology was developed to model the concept of the epilepsy patient profile, namely ESO 'Epilepsy Seizure Ontology'. This was a driving force for the ultimate PMP Framework and a critical aspect for this concept. In order to make ESO useable for HCPs (Health Care Professionals) the ontology was transformed into a language that is understandable by humans and machines, this was accomplished by XML and the outcome was PPDL (Patient Profile Description Language). 
- Distinct groups of epilepsy patients that share similar characteristics using Clustering Analysis. This will enable the health carers to define a 'type' of epilepsy patient.

This has been achieved by performing clustering analysis techniques. Different clustering techniques were initially analysed to find the most appropriate approach for the acquired epilepsy data and an in-depth focus upon 'clustering considerations' was undertaken to confirm validity. The outcome was a set of 6 distinct 'clustering' groups, these 6 cluster groups revealed six completely different categories of patients each with their distinct seizure related information.

- A novel IoT framework to realise "Personalised" monitoring of patients.

This has been achieved by informed experimental results in the development of the conceptual PMP Framework which evaluated the assumption that an individual patient profile or distinct group of patients sharing similar characteristics can make a difference in which IoT device to use. The experiments were performed to capture seizure data, obtained from sensors, which were positioned on different parts of the patient's body. The experiment outcomes revealed that difference is observed due to sensor 'position' on the body and that sensor 'types' are suited to different patient profiles.

- An original 'Research Design Framework'

Incorporating a sequential strategy, the research design approach used in this thesis originated on findings from the literature review, however it is a new and novel way to achieve the type of 'personalisation' described in this thesis. The framework is shown in the Methodology, Chapter 3 (Figure 3.1) and this design approach is transferrable to other studies aiming to achieve personalisation within alternative domains or can be applied to other health conditions such as diabetes, asthma or a condition with many characteristics.

\subsubsection{Thesis Structure}

The rest of this thesis is structured as follows:

Chapter 2, 'State of the Art' presents an in-depth review of the literature context of this study on several areas including epilepsy and healthcare monitoring systems, technologies to help 'detect' seizures and the challenges identified with epilepsy detection and monitoring systems. The concept of Personalised Healthcare is introduced with an in-depth discussion on methods to make epilepsy patients distinct from one another.

Furthermore, this chapter will progress with a critical investigation of the literature of the concept of using semantic ontologies and its relevance to matching a patient profile with a 
device profile. Finally, the chapter ends with a detailed understanding of why the modern trend is toward the personalised healthcare.

Chapter 3 presents the research methodology of this study. It explains the research design and methods selected in order to achieve the aim. Methods and techniques adopted to extract the data, create the ontology, perform K-means Clustering are discussed. A framework of the research design containing the study objectives is systematically discussed at each stage and the various methods tools and techniques used discussed in detail.

Chapter 4 presents the steps taken to gather the knowledge to inform the PPDL (Patient Profile Description Language). To do this the development of the ontology: ESO 'Epilepsy Seizure Ontology' is discussed. From the knowledge discovery process right through to the ontology implementation and evaluation this chapter presents the authentication of the ESO demonstrating its features and how it is used to create an individual representation of a seizure type patient, and ultimately enlighten the PPDL 'Patient Profile Description Language.'

Chapter 5 begins with an overview of clustering analysis and discusses the justification to fulfil Objective 3 and hence cluster the patient profiles. This chapter proceeds to systematically discuss the steps described in the research design framework to perform Kmeans Clustering. From preparing the data needed right through to its visualisation and evaluation this chapter describes in-depth how the clusters are constructed and finally reveals a list of typical seizure type patients and which cluster they belong to.

Chapter 6 discusses the experiments performed to test the thesis hypothesis. The results from the experiments will be used to inform the framework discussed in the next chapter. This chapter will discuss and analyse the results from the test carried out.

Chapter 7 is where the evaluation takes place. It will focus on the development and of the proposed PMP framework to do this. The chapter will continue to outline and discuss the components of the proposed PMP framework then finally test the framework with 'epilepsy scenario' use-cases taking the user through each step of the framework.

Chapter 8, 'Conclusion and Future Work' summarises the overall research work that has been done to achieve the aims and objectives that where originally identified in this study. With recommendations based on the findings of this research, also an understanding and justification of how an "IoT Approach to Remote Personalised Monitoring and Management of Epilepsy" successfully works with clear measurable attributes found in the use of the PMP framework.

\subsection{List of Publications}

Conference: The Second International Workshop on Mobile Technology for Healthcare (MT4H2017)

Paper: An IoT Approach to Personalised Remote Monitoring and Management of Epilepsy 


\section{Chapter 2 State of The Art}

\subsection{Introduction}

The emphasis of this chapter is to introduce relevant concepts and challenges that are considered to be influential and important to the development of this research. It begins by discussing approaches to healthcare monitoring systems, particularly recent 'smart environment' approaches. The next section focuses on monitoring specifically for epilepsy and technologies to help 'detect' seizures together with an in-depth discussion of the challenges identified. A summary table is presented with an overview of the all the 'smart' approaches discovered in this chapter. Finally, this chapter analyses the concept introduced in Chapter 1 on making epilepsy patients distinct from one another by introducing 'Personalised Healthcare', continuing with focus on methods such as 'clustering analysis' to address patient individual parameters, following which there is a review on methodologies for ontology development.

\subsection{Healthcare Monitoring Approaches}

In the history of time it is only relatively recently that computers began to assist healthcare monitoring, in 1950s' patients began to be continuously monitored by computerised machines [28] and clinical monitoring was first envisaged in the home [29]. For computer assistance to epilepsy it was not until 1972 in the field of imaging, when computerized tomography (CT) was invented by the British engineer Godfrey Hounsfield [30] and only in recent decades where specific epilepsy healthcare 'monitoring systems' have been proposed by researchers, some of which are discussed in this chapter and shown Table 2.2. Smart Approaches to Monitoring in Healthcare and Epilepsy.

Much of this recent growth being due to the advent of current IoT technology whereby the rise of 'smart environment' approaches to healthcare monitoring is witnessed. Such smart environment approaches are also shown in Table 2.2 where approaches are summarised accordingly following discussion, under: 'Healthcare Monitoring', 'Epilepsy Monitoring', 'Epilepsy Apps' and 'Epilepsy Sensors'. These recent 'smart' approaches in healthcare demonstrate the trend toward 'sensor use' and 'remote monitoring'.

Researchers are bounding toward the new generation of smart technology and IoT (Internet of Things). Novel devices such as smart watches, smart bands \& smart clothing are all competing for the ultimate solution. Yet it is found there is limited research which focuses upon the concept of a more holistic, personalised approach to help manage epilepsy. 
One study deemed the significance of attention on smart technologies and its potential to identify early indicators of cognitive and physical illness [19] and observed that researchers have argued and predicted that assessing individuals in their 'everyday environment' will provide the most 'valid' information about everyday functional status [31]. Indeed, there is evidence recently of this indication as several IoT platforms to manage \& monitor healthcare remotely, are observed. For example, one IoT paradigm comprising of Wireless Health Sensors (WHS) permits the continuous monitoring of biometric parameters such as pulse rate, pulmonary functional quality, blood pressure and body temperature [32]. This IoT paradigm is being used to assist predictive analysis via smart healthcare systems by a medical practitioner. Using sensors connected to Arduino patient status is tracked, and by a Wi-fi connection data is collected and transmitted and can receive user requests. This data is shared with doctors through a website where the doctor can analyse the condition of the patient and provide further details online and intimate patient about future severity well in time [32].

There are several monitoring approaches specifically for Epilepsy which are discussed in section 2.3, but first this literature review will focus upon approaches which manage other health conditions: for example one study examines a Smart Environment for children with Autism and theirs was the vision of the school clinic seamlessly embedded and connected through advanced communication technologies creating a highly interactive physical world furnished with sensors, actuators, and novel displays [12]. This Autism experiment identified the sustained use of smart environments, focusing on models for predicting behaviour through the proposed generation of rich databases required for the continuous tuning and personalisation of classifiers. This study is a good example of one of the early smart environments used in healthcare and identifies that personalisation can be used to predict behaviour.

Finding accuracy in predicting, diagnosing and recommending are the desired goals as observed in one study whereby an ontology-based model for diagnosis and treatment of diabetes patients in remote healthcare systems (OMDP) was proposed and experimental findings suggested more accurate findings for management of diabetes compared to other medical applications [10].

Another noteworthy system is ROCHAS (Robotics and Cloud-assisted Healthcare System for Empty Nester) [33] which recommends the monitoring of disabled patients in their own home, this provided them with robotic assistance but also allows them to live as independently as possible. Similarly, an assistant platform for elderly people was proposed in a study [34] whereby open software platforms are analysed to work together.

The use of 'sensor data' in many more healthcare systems is becoming widespread, for example body temperature is captured in weblogs to monitor unhealthy children in 'The Chinese CMTHC' project, a Children's medical treatment and healthcare system [35]. Wearable devices with embedded sensors are becoming more commonplace notably: 
AIWAC which proposes affective interaction through wearable computing and cloud technology [36] this analyses the affective needs of the patients based on the measurements obtained from a wearable device.

Allergy and Asthma Care in the Mobile Phone Era (or known as AACMPE ) [37] makes use of gravity sensors and controls the allergies and asthma evolution of the patients by means of Mobile Cloud Computing to determine several variables: the peak exhalation flow and the peak nasal inhalation flow and some breath parameters and sounds.

Other computing studies conducted in healthcare vary; i.e. how to wirelessly transmit patient data from a device into the existing healthcare systems [38] whilst others simply discuss the positive effects of this synergy between Semantic Web and healthcare processes [39]. Furthermore, it was unfortunately discovered that instead of using semantic technologies many existing datasets in healthcare environments are still stored in relational databases [40] making data mapping a difficult process.

The development of semantic technologies is emerging in popularity for healthcare, by combining real-time user-generated data and domain expert knowledge they are used for modelling relevant information, and for fostering reasoning activities. Many researchers are proposing the use of Semantic Web technologies to build a system for supporting and motivating people in following healthy lifestyles [41].

Consequently, as medical data is rapidly growing [42] the idea of using Semantic interoperability can provide a positive and meaningful exchange in the use of clinical data between many healthcare systems [43]. Yet some obstacles with healthcare data are known to slow this growth since healthcare data is generally heterogeneous, distributed, and nonstructured because each data element can have its own schema, structure, standard, format, coding system, level of abstraction [42].

Another challenge coming from healthcare applications is the handling of all the information from the flourishing data created by IoT devices, [44] thus Carbonaro et al present an ontology-based system for the ehealth domain which provide semantic interoperability among heterogeneous IoT devices and facilitates data integration and sharing [44].

\subsection{Epilepsy Detection \& Monitoring Systems}

In 2010 'A Knowledge Management System’ [45] was developed for supporting creation, capture, storage and dissemination of 'information' about Epilepsy and Epileptic Seizures. The ontology on Epilepsy and web-based prototype to create the KMS (Knowledge Management System) is interesting as the issue of adding new concepts to the ontology was identified during the development, whereby the significance of continual communication with medical doctors was highlighted signifying the importance of understanding the 
respective meaning of ontology concepts and to try to find new applications for the ontology, serving the patients, doctors and researchers [45]. This highlights a gap and calls for new applications for the epilepsy ontology, could new evidence found in more recent studies, hence 'personalised healthcare' contribute? As depicted, the ontology should reflect this evolution since the field of epilepsy and epileptic seizures is continually evolving [45].

More recently one study focus on Epilepsy symptoms particularly those that 'lead' to the seizures, are identified as being quite uncertain and there is the possibility they can unfortunately go undetected [46]. A Transition Model between the different phases of epilepsy to be adopted by decision-making systems used by people who are diagnosed with the specific disorder is proposed. Based on the whole model's transitions information, epilepsy is represented by a health caring ontology which contains all the useful aspects of it [46].

University of Freiburg's Cluster of Excellence BrainLinks-BrainTools studied smart devices to interface with the brain, were a "Closed Loop Stimulation System for Epilepsy Therapy" was proposed and a promise of new chances for patients suffering from pharmacoresistant epilepsy. The study used animal experiments and obtained an insight into optimal parameter settings since identifying that electrical brain stimulations are complex and poorly understood, this is a good example of how prediction methods were tested [47].

EEG, an electroencephalogram is a recording of brain activity. This is the chief gold standard method used within hospitals to detect and monitor seizures. Several approaches have been reported with the aim to embed this method in other settings and platforms. Developments in some topics have been published, such as modelling the recorded signals [48] [49]or the design of portable EEG devices to deploy such models. As an alternative and sometimes supplement to EEG there exist many sensors embedded in clothing or worn on the body to obtain bio-signals such as gyroscopes, accelerometers, pulse rate, temperature sensors, magnetometers, galvanic skin response sensors (GSR), implanted advisory system, electromyography, video detection systems, mattress sensor, and audio systems [50].

The epilepsy specific platform such as Sareen (mentioned in Chapter 1) also makes use of data from EEG and uses Mobile Cloud Computing (MCC) for storage and for sending notifications to the family and clinicians [51]. MCC is being embraced in other epilepsy platforms such as EpiCare, where it is used in home settings specifically to assist epilepsy diagnosis [2], whilst body sensor networks placed on the body together with MCC has been proposed for epilepsy detection whereby [1] body sensors such as pulse rate which can be worn as a wrist band in a recent platform: 'An IoT Platform for Epilepsy Monitoring and Supervising' [1].

At the time of writing this chapter an initial review was done to discover existing sample devices for epilepsy. Variation in the available devices and their purpose was observed, i.e. in diagnosis, prevention, detection or monitoring. The sample devices are noted in Table 2.1. 
Diversity in Epilepsy Devices, some devices are already in use in the home: i.e. mattress sensor devices whereas EEG, VNS (Vagus Nerve Stimulation) and brain stethoscope devices are used in hospital setting only and need medical training to use. Not all the devices use IoT technology or sensors. These initial findings highlighted the diversity in management of epilepsy and indicate how different devices suit specific needs in different epilepsy scenarios. Also noted were the benefits and limitations for each device, see Table 2.1. Diversity in Epilepsy Devices.

A large amount of apps have been published more recently especially in the commercial sector for the detection and management of seizures using either the Smartphone sensors or external sensors, for example Epdetec [52] and Myepipal [53] and web logging which facilitates the way a patient records daily information concerning her/his epileptic events, medication, and news, My Epilepsy Diary [54] and Epidiary [55]. Another app attracting attention and recently reviewed in the press is the Alert App by Empatica. This app sends caregivers an automated SMS and phone call when it detects unusual patterns that may be associated to a convulsive seizure [56] yet it is only designed to work with the Embrace Smartband by Empatica and can prove expensive for the user [57]. 
Table 2.1. Diversity in Epilepsy Devices

\begin{tabular}{|c|c|c|c|c|c|c|}
\hline $\begin{array}{l}\text { Epilepsy } \\
\text { Devices }\end{array}$ & $\begin{array}{l}\text { Placement/ } \\
\text { Situation }\end{array}$ & $\begin{array}{l}\text { Use at } \\
\text { Home }\end{array}$ & Purpose & $\begin{array}{l}\text { Medical } \\
\text { Training } \\
\text { Needed }\end{array}$ & Benefits & Limitations \\
\hline $\begin{array}{l}\text { EGG (Gold } \\
\text { Standard) }\end{array}$ & Brain & No & Monitoring & Yes & $\begin{array}{l}\text { Ability to see real } \\
\text { time brain } \\
\text { activity }\end{array}$ & $\begin{array}{l}\text { High cost and limited } \\
\text { commercial } \\
\text { availability }\end{array}$ \\
\hline Smart Belt & Torso & Yes & Detection & No & $\begin{array}{l}\text { Comfortable } \\
\text { (worn under T- } \\
\text { Shirt) }\end{array}$ & Restricted to Torso \\
\hline $\begin{array}{l}\text { Vagus Nerve } \\
\text { Stimulation } \\
\text { (VNS) }\end{array}$ & $\begin{array}{l}\text { Nerve in } \\
\text { neck }\end{array}$ & Yes & $\begin{array}{l}\text { Diagnostic/ } \\
\text { Prevention }\end{array}$ & Yes & $\begin{array}{l}\text { Reduces } \\
\text { frequency of } \\
\text { seizures }\end{array}$ & $\begin{array}{l}\text { Generally invasive as } \\
\text { implanted under skin } \\
\text { and expensive }\end{array}$ \\
\hline $\begin{array}{l}\text { Brain } \\
\text { Stethoscope }\end{array}$ & Brain & No & $\begin{array}{l}\text { Detection/ } \\
\text { Monitoring }\end{array}$ & Yes & $\begin{array}{l}\text { Able to } \\
\text { detect non- } \\
\text { convulsive } \\
\text { seizures }\end{array}$ & $\begin{array}{l}\text { Cumbersome, not yet } \\
\text { widely commercially } \\
\text { available }\end{array}$ \\
\hline $\begin{array}{l}\text { Medpage }^{\mathrm{TM}} \\
\text { Model MP5 }\end{array}$ & Mattress & Yes & Alarm & No & $\begin{array}{l}\text { Contact free } \\
\text { Video }\end{array}$ & $\begin{array}{l}\text { Expensive and } \\
\text { limited to sleep }\end{array}$ \\
\hline Emfit & Mattress & Yes & Alarm & No & $\begin{array}{l}\text { Contact free } \\
\text { Video }\end{array}$ & $\begin{array}{l}\text { Weight limit. } \\
\text { Expensive and } \\
\text { limited to sleep. }\end{array}$ \\
\hline Smartwatch & Wrist & Yes & $\begin{array}{l}\text { Monitoring/ } \\
\text { Alarm }\end{array}$ & No & $\begin{array}{l}\text { Wearable and } \\
\text { affordable }\end{array}$ & $\begin{array}{l}\text { Battery life, lack of } \\
\text { developer support }\end{array}$ \\
\hline Embrace & Wrist & Yes & $\begin{array}{l}\text { Monitoring/ } \\
\text { Detection }\end{array}$ & No & $\begin{array}{l}\text { Multiple } \\
\text { detection } \\
\text { modalities and } \\
\text { wearable }\end{array}$ & $\begin{array}{l}\text { Expensive and long } \\
\text { charging time. } \\
\text { No extendible straps } \\
\text { supplied to fit to } \\
\text { ankle/leg }\end{array}$ \\
\hline Sami & Surveillance & Yes & $\begin{array}{l}\text { Camera/ } \\
\text { Video } \\
\text { Monitoring }\end{array}$ & No & $\begin{array}{l}\text { Immediate Play- } \\
\text { back and contact } \\
\text { free }\end{array}$ & $\begin{array}{l}\text { Limited to sleep and } \\
\text { high false alarm rate }\end{array}$ \\
\hline
\end{tabular}


An excellent performance comparator of epilepsy related apps is proposed by Ranganathan et al [58] and this study even goes as far to demonstrate the ideal monitoring system for epilepsy and indeed deems the system in Figure 2.1 [58] to be the "Holy Grail of Epileptology".

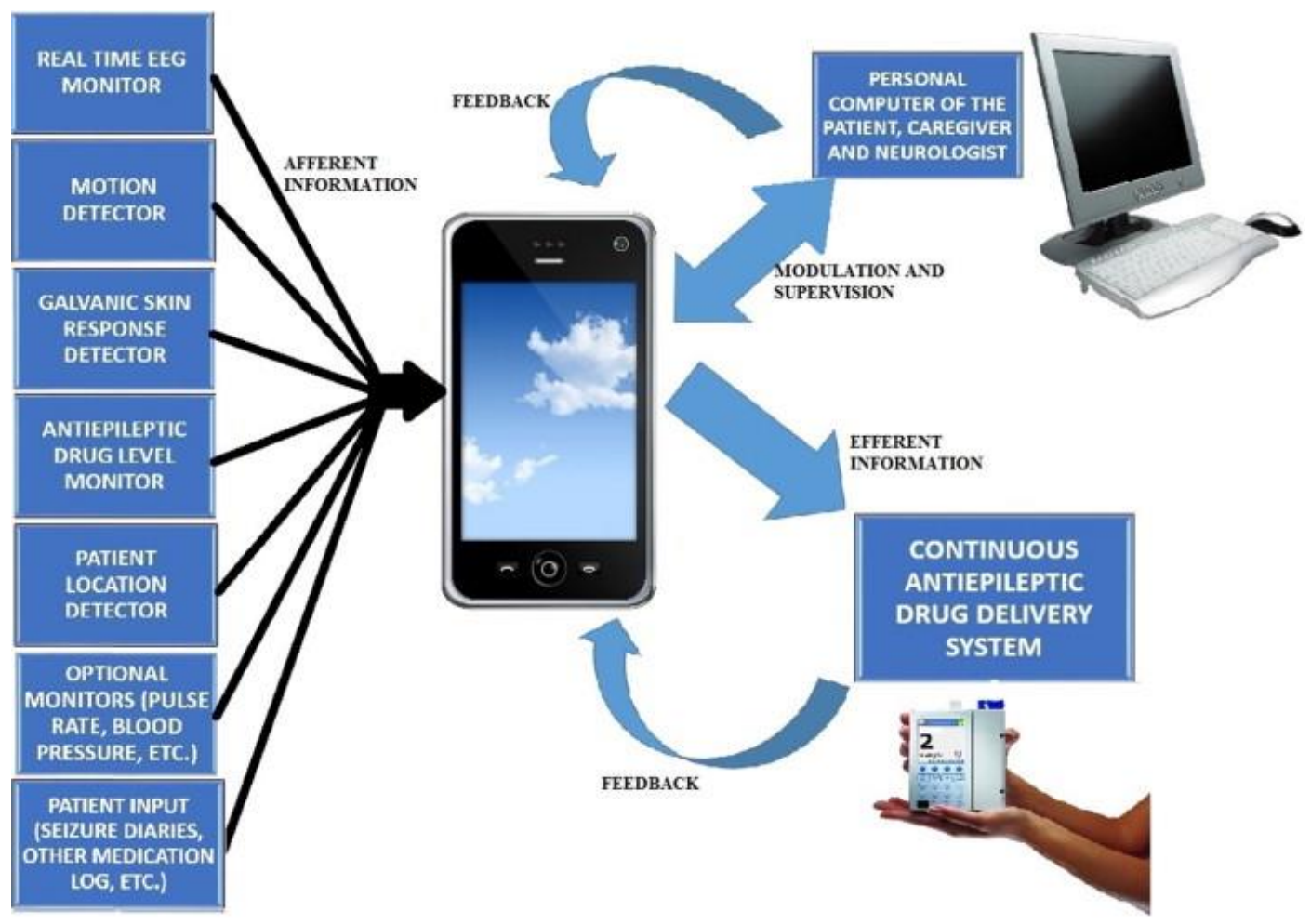

Figure 2.1 Holy Grail of Epileptology

This system above encompasses a holistic approach: sensors are embedded on the patients either clothing or within the smart phone to capture all manner of data from galvanic skin responses, to location, blood pressure and even real-time EEG monitoring. The mass variety of information captured in the phone feeds back to the patient, career and neurologist alike and the drug delivery system is administered. This envisaged system goes some way to painting a picture of the individual epilepsy patient, but it does not distinguish between one epilepsy patient or from the other nor address the types of seizures or the frequency of seizures from the patient.

Table 2.2. Smart Approaches to Monitoring in Healthcare and Epilepsy

\begin{tabular}{|l|l|l|l|}
\hline Healthcare Monitoring & Epilepsy Monitoring & $\begin{array}{l}\text { Epilepsy } \\
\text { Apps }\end{array}$ & Epilepsy Sensors \\
\hline Wireless Health Sensors (WHS) & $\begin{array}{l}\text { Epilepsy Knowledge } \\
\text { Management System }\end{array}$ & Epdetec & Gyroscopes \\
\cline { 1 - 2 } $\begin{array}{l}\text { Smart Environment for children } \\
\text { with Autism }\end{array}$ & $\begin{array}{l}\text { Transition model for } \\
\text { symptoms }\end{array}$ & Myepipal & Pulse rate \\
\hline
\end{tabular}




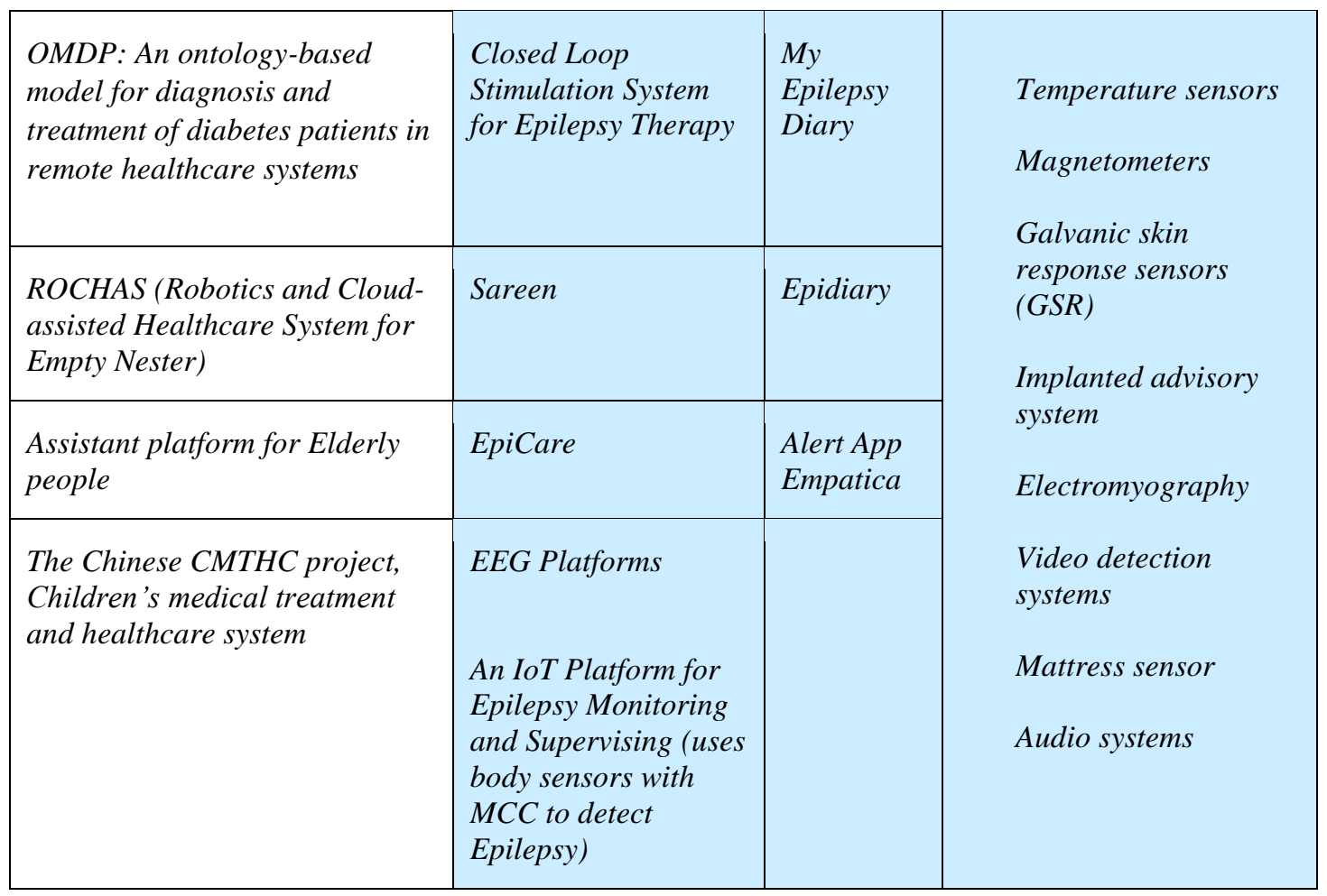

\subsection{Personalised Healthcare and IoT (Internet of Things)}

Instead of being handed a leaflet from your clinician which explains your condition in general what if you could be presented with an individualised personalised plan? This plan could be made up specifically for your specific strand of the condition, tailor made based on your characteristics, patients could walk out of the surgery with a personalised assessment of their health and have their own personalised disease management plan [43]. Researchers are recognising there are changes coming in healthcare, which can endorse the meaningful use of electronic health care data, and patient-centered outcomes, even in 2013 the need for personalisation was becoming recognised noting that the timing has come about for this kind of research direction [43].

Recently personalised healthcare is known to be facilitated by Internet of things (IoT). As endorsed by one of the major mobile providers in the UK identified is that IoT devices could provide patients suffering with long-term illnesses with access to more personalised treatment [59]. Hence these wearable devices which allow physiological signals to be continuously monitored at home can help to resolve the problem of occasional visits to the surgery that can only offer a transitory 'window' into the physiological status of the patient [60].

Personalised healthcare and IoT is becoming popular emphasised again with the recent advancements of wearable devices and smartphone, this is since the fast explosion of wearable devices, smartphone and IoT enabled technology. This technology is evolving healthcare from old fashioned hub based systems to more personalised healthcare systems (PHS) Personalised Healthcare Systems [61]. Therefore, IoT can be deemed as game changer 
by endorsing personalised healthcare, because with these connected devices we can also achieve self-tracking whereby make way for a future where individuals will be more involved in the management of their own health and data will be generated that can benefit clinical decision making and research [62]. As key players in the move toward personalised healthcare IoT devices are thus attracting keenness from medical and public health professionals [63].

The idea that health conditions should be treated by recognising the 'multiple factor' elements, or the strands of the condition is beginning to come to light. For example in recent years it is becoming more apparent to treat organ monitoring systems by providing care by organ specialty. A healthcare delivery model that is personalised to achieve individual health outcomes is needed since chronic disease involves multiple health factors with complex interactions between them over a prolonged period of time [63]. Another technique in line with personalised monitoring deemed immensely useful for the management of important chronic health conditions, such as diabetes, obesity, depression, ageing and mental health is to determine the 'parameters' of the individual, this is achieved by the personalised monitoring of health by the determination of weight, activity, sleep, heart-rate, blood glucose diet and other parameters [64].

A further aspect of personalised healthcare identified is systems which provide tailored recommendation capabilities. Work done Ivanović $\mathrm{M}$ et al uses agent technologies to support healthcare cases and found this revealed ways to discover new applications such as personalised and socialised healthcare platforms and interestingly systems with tailored recommendation capabilities [65]. In order to discover the patient individually these new technologies enable opportunities to discover the patient's preferences, traits, and states. This highlights the trend toward the focus of the patient as an individual, and requests healthcare plans which are specially tailored for these preferences, traits and states.

\subsection{Personalised Healthcare for Epilepsy}

Certainly the very complex nature of Epilepsy can lend itself to a personalised approach; because there are over 40 different types of seizure [66], in addition there is an enormous 'variety' of behaviours that may occur in different types of seizures and furthermore seizures are usually; 'Stereotypic' meaning symptoms are similar whenever they occur [66]. An individual 'seizure type profile' or an individual 'patient dataset' can be realised and hence a personalised approach to treating epilepsy is assumed viable.

Accordingly, the concept of providing personalised tailored recommendation capabilities could be applied to epilepsy. If the precise IoT smart device is matched to the precise 'particulars' (based on their seizure indication and type) of the patient profile and continuously monitored then not only are the chances of capturing seizure data greater, but a 
tailored healthcare plan could be provided to the patient which recommends that device. Ultimately, such a personalised environment could help those who might occasionally miss an epileptic event and make enhancements for carers performing their overall monitoring scheme [67].

As noted previously in this chapter personalised healthcare also embraces determining the 'parameters' of the individual [68]. Therefore, it is appropriate to focus upon the parameters that make epilepsy patients distinct from each other in this thesis, this is addressed in the ontology development in Chapter 4. One example is 'seizure types'. The two most common types of seizure are generally described in two major groups generalised seizures and focal seizures (or partial seizures), the difference between the two is in how and where they begin in the brain [69]. Another parameter particular to an individual epilepsy patient is whether there is any movement during a seizure, when no motor symptoms happen, it is known as a non-motor seizure [70]. These parameters are just a few amongst many that can be used to identify the individual, the sheer diversity of epilepsy is commonly known and it is recognised that it is not one singular condition and that seizures occur frequently with a an essentially wide range of seizure types, frequency and severity [68].

Similarly, during a survey on current seizure detection and classification technologies, Bidwell et al also found a need for better distinguishing between patients exhibiting generalised and partial seizure types and in turn achieving more accurate seizure counts [71]. Another study recognised that there was little available information on which combination of bio-signal or bio-signals is the best for individual seizure types and for each individual patient [72]. Here it is evident that 'seizure types' are addressed; these observations provide further evidence and endorse the fact that epilepsy patients are so different from each other and how there is a need to focus upon each individual and their 'seizure type' and to actually individualise the seizures.

In addition to academic research, some non-academic sources suggest personalised monitoring of epilepsy [73]. For example, Niesm, an Australian company reveal a personalised epilepsy monitoring system. As with other gurus, they recognise that the current way of managing epilepsy is as in UK with video monitoring in hospital settings and with the gold standard EEG. They propose a solution using their devices whereby patients wear a watch device and seizures are captured continuously and data is recorded at their NIESM portal.

Niesm highlight the current trend towards personalisation in their proposal where they deliver a personalised epilepsy monitoring system combines the latest advances in wearable technology with quantitative data analysis [73]. The personalisation aspect characterizes patient seizure activities over extended times, and their holistic approach uses machine learning to create personal patient profiles. This is a good method to achieve personalised healthcare using machine learning. These perceptions which find specific solutions for 
seizure types [73] and recognise that other products do not embrace this, see Figure 2.2 which provides a good opportunity for researchers to find other methods to achieve this.

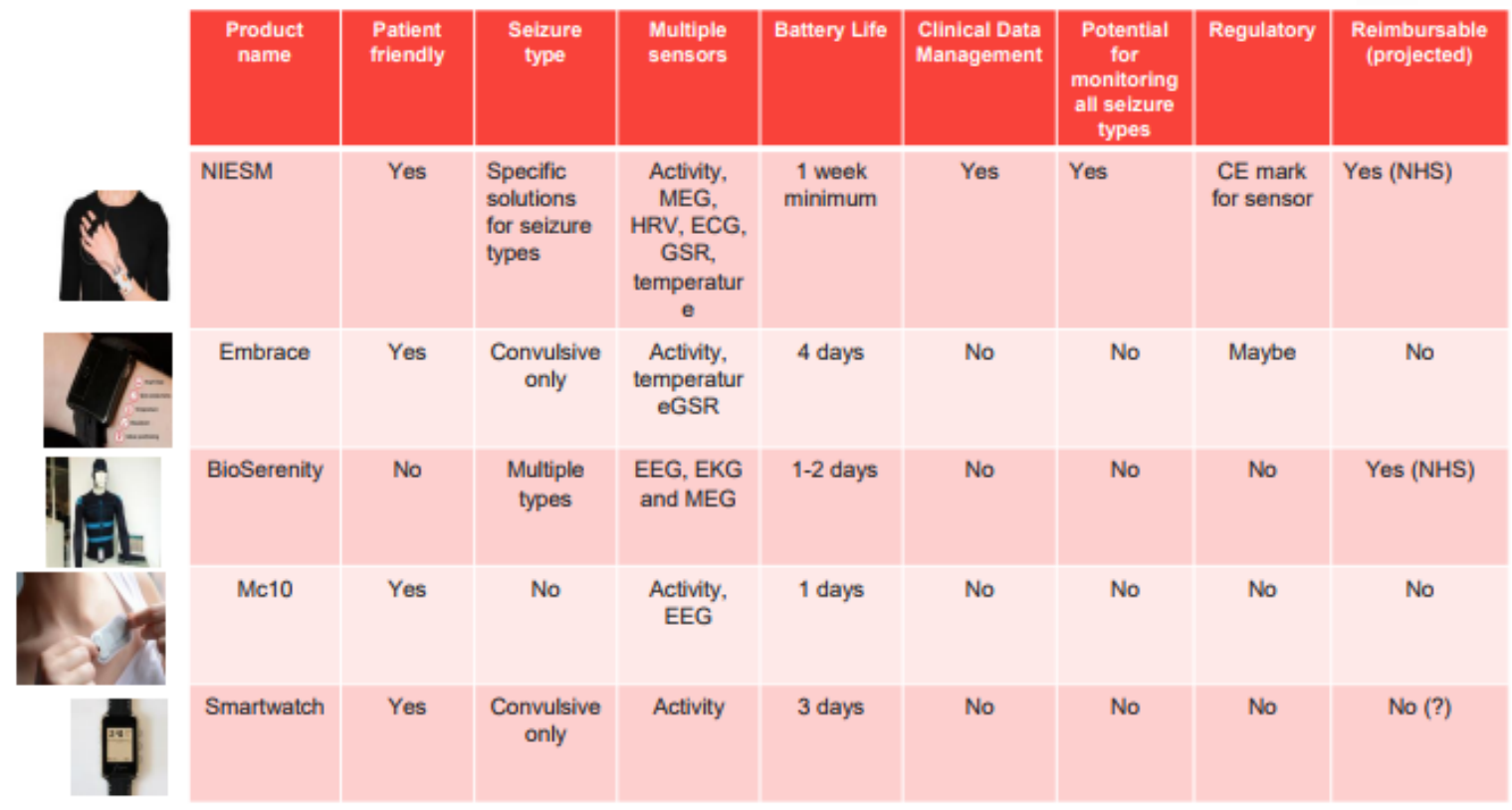

Figure 2.2. Competitive Sensor Landscape

\subsection{Addressing Current Gaps}

Studies discussed in this chapter have revealed many IoT approaches for the monitoring and management of epilepsy many of which encompass a network of connected smart devices which are equipped with sensors either embedded in clothing or smart phones, to either detect, predict or manage epilepsy. Discoveries disclose how IoT is utilised to support the ever-growing trend of personalised healthcare.

However, the monitoring approaches and systems uncovered in this review do not focus on the embracing of a truly personalised approach for epilepsy. Even though the 'diversity' of epilepsy is acknowledged and has been identified in other studies, i.e. by highlighting the importance of distinguishing each 'seizure type', there is still a gap to address such parameters. The 'seizure type' is just one of many parameters that can distinguish one seizure patient from another. Therefore, these very individual characteristics can be further identified to address the challenge to achieve a truly personalised approach to managing epilepsy.

More so recently researchers are recognising, that devices should specially take into account the user's seizure types and personal preferences, [74] focus should be shifting not only on the desires of the users but seizure detection devices should be able to 'adapt' to the patient's characteristics and seizures [74]. Regrettably, there are few specific sensor detection options for each specific seizure type, this is an imminent requirement for patients and their carers. Ideally when choosing a seizure detection device, the patient-specific seizure semiology's should be considered [3]. Thus, highlighting the need for a type of monitoring that 
distinguishes one patient from another and depicting the need for devices to pinpoint the patient-specific signs and symptoms.

Primed from the literature analysis this thesis will begin with a focus upon the parameters that make epilepsy patients distinct from each other. In addressing the challenge to achieve this 'personalised' approach to remote monitoring system for epilepsy, other parameters must be found, so that the individual patient profile can be realised. Furthermore, it is important that specific sensor-based devices are identified to detect the individual parameters.

To achieve this objective firstly the domain in question which is the 'epilepsy patient' must be understood, this will be achieved by developing an ontology.

To begin the ontology development a suitable methodology should be selected which should begin with a 'knowledge acquisition stage' [75]. Part of this initial literature review aims to survey and discover the best methodology to use for the development of the ontology language. Section 2.6.1 shows selection process for a suitable method.

Another aid toward personalisation is the concept of clustering. Recent discoveries in literature unveil focus upon clustering patient profiles. For example, in 2019, Boeva et al focus on, the selection of a personalised treatment plan for a patient by using a split-merge evolutionary clustering algorithm, that can be used for maintaining patient profiles in healthcare [76]. In using clustering techniques, it was discovered that in associating patientspecific disease characteristics the person's increased risk for cardiovascular disease can be identified [77].

Similarly, a cluster-based approach is highlighted recently for patients with insomnia and considered a possibility to enhance patient care by guiding tailored patient-centered management of patients presenting with insomnia [78].

A mental health clustering tool released 2019 is used as a needs assessment tool designed to rate the care needs of a patient,[79] it uses rating scales, yet clinical human judgment is still needed, as observed in the NHS improvement publication when addressing HCP's; they recommend that the final clustering decision is still the clinicians based on their assessment results in applying this guidance (derived from clustering) [80], thus demonstrating user intervention is still needed.

The prevalence of clustering patient profiles in different domains in healthcare is becoming apparent particularly in recent times: another 2019 study is noticed for use in detecting drug use patterns and Klaid et al observe that in their knowledge this is the first study proposing cluster analysis for detecting drug utilisation patterns from electronic healthcare records [81]. Another clustering analysis study is discovered on acute decompensated heart failure (ADHF) where authors found that their results may be superior to bedside classifications [82]. This indicates the recent success of clustering. 
Yet the only real value and purpose for clustering patient profiles is so that their treatment can be personalised, since every patient is capable of showing a different response to standard treatment procedures even for a well-defined clinical condition [83]. Likewise, as witnessed by Payne-Murphy and Beacham in 2014 when profiling chronic pain patients, the research pathway of tailoring personalised interventions based on the cluster profiles is highlighted when they identify clusters with significant differences among them [84] thus indicating the relevance of clustering in personalised healthcare.

Yet there is limited evidence on clustering for epilepsy patient profiles and hence the discovery of profiles for epilepsy patients is an open question that needs addressing. Consequently, this thesis will address this gap drawing on knowledge from the ontology development in Chapter 4. Deeper analysis of the concept of clustering analysis is undertaken in Chapter 5 of this thesis.

\subsubsection{Ontology Development Methods}

In finding the ideal method to use for ontology development, this investigation/exploration of the literature demonstrates that there is no gold standard method for developing an ontology as witnessed by Noy \& McGuinness in the claim that there is no single correct ontology-design methodology [75].

In the publication "Guide to Creating your First Ontology" [75] the focus is upon a simple knowledge engineering approach and uses 'a good rule of thumb' to provide a guide. It is discovered that the best solution almost always depends on the application that you have in mind and the anticipated extensions [85] this makes good sense due to the fact that each domain and scope of project is so different.

Most methodologies achieve the same goals with a first stage usually named 'data gathering' whereby key terms or keywords are identified. Noy \& McGuinness call this the 'Textual Analysis Approach' and such initial decisions should be made which consider the key terms to be talked about and their associated properties. Whereas another study phrase this stage as 'Information Extraction' [86] and claim this stage is not a mere keyword filtering method; they use more precise steps and bring in linguistic analysis derived from Hobbs et al. [86]:i.e. text pre-processing, whose level ranges from mere text segmentation into sentences and sentences into tokens to a full linguistic analysis.

Some researchers focus on Knowledge Acquisition Techniques used for ontology development [87]. Data gathering here involves interacting with domain experts to extract all of the required knowledge; gaining the vocabulary required for assisting in ontology model development [88]. Also defining a Glossary of Terms (GT), a group of tables to encompass types, classes, associations, attributes, and instances. 
Boicu et al aptly describe ontology development as building a knowledge base and they assert that it is always a difficult task to start from scratch. Consequently, it would make more sense to try to reuse knowledge than to recreate knowledge [89]. Moreover, this reuse should also facilitate the communication between the systems because of their shared knowledge.

\subsubsection{Text Mining Approach}

Continuing the focus on the data gathering phase and the extraction of key terms a study by Tsoi et al looks at a text mining approach to ontology development in Bio-medical Science. It is identified that it still poses significant challenges developing such high-quality ontologies, as a wide range of domain experts need to be involved [90]. To aid ontology development they survey numerous methods that have been developed to extract terms from literature automatically. Tsoi et al find that these automatic extraction methods are hardly ever used despite these efforts, and that it is still widely recognized that 'manual curation' is the most reliable method for ontology development, therefore the chosen methodology for this research will adopt this approach as observed later in Chapter 3, Methodology.

Tsoi et al acknowledge (as do researchers identified above) that it is a substantial challenge to determine which terms should be used as the basic building blocks to develop the ontology; interestingly they acknowledge that some objective method and measure is used without either a preliminary or confirmatory test conveying that the term selection process really relies on the expertise of individual curators, and they endorse that a quantitative approach to evaluate whether terms are suitable to develop an ontology for a particular domain would provide this objective method and measure, improve the usefulness of the resulting ontology, and lessen the amount of work imposed on curators [90].

A similar approach to text mining is text clustering which is adopted by Rajput 2017 [91] although it does enable text-mining and some text extraction techniques. Rajput present a clustering ontology based text mining for grouping paper or proposals and assigning that grouped proposal to reviewers systematically. Again the challenging nature it is advocated; in the issue to find accurate knowledge, information (or features) in text documents to help users to find what they want and what data they want. Rajput find that most existing text mining methods adopted term-based approaches suffer from the problems of polysemy and synonymy and that generally traditional text mining has a problem with polysemy and synonymy. Yet the popularity of text mining is growing due the fact it can automatically discover the knowledge assets buried in unstructured text [91].

\subsubsection{Sequential Ontologies}

Indeed, there is a significant challenge in constructing a well-developed and sharable ontology to address the research questions. 
Since investigating many authors, it is apparent that there is range in the way ontologies are designed. Some of these methodologies and approaches have been reported in the literature regarding ontology building methodologies. However, there is ongoing argument within the ontology community about the best method to build them [92].

A study in 2015 [93] adapted methodologies to suit the purpose of coding historical documents, in this study the ontology building process was divided into the following stages:

- $\quad$ Identifying the purpose, scope, and users

- Domain analysis and knowledge acquisition

- $\quad$ Building a conceptual (informal) ontology model

- $\quad$ Formalization

- $\quad$ Evaluation In our method, we focus on an evolving prototype of the ontology

This sequential (step by step) process will be utilised and adapted for this research, to ensure robustness it is observed that at every stage of the development process there is specific criteria that must be adhered to such as clarity, coherence, extensibility, minimal encoding bia and Minimal ontological commitment [94].

Another sequential methodology used by Sampalli in 2011 [95] uses a generic framework for the development of an ontology and is divided into three important phases: a specification phase, a conceptualization phase, and an implementation phase. The generic steps and the variations that are involved in the development of an ontology in a heterogeneous domain such as a controlled vocabulary and involvement of domain experts is key to ontology development.

Further sequential methodologies exist again demonstrating the stages of ontology development, this time comprising of with 4 phases: data gathering, data analysis, ontology development and testing [96]. Similarly, stages are similar to other methodologies used such as and stages can be adapted to suit its purposes and built from scratch if necessary [97].

\subsection{Summary}

Evidence provided in this chapter showed that personalised healthcare is facilitated by IoT within industry yet there are very few experiments and limited evidence of how IoT sensorbased devices are used in hospital settings. Consequently, could IoT be a game-changer in endorsing a personalised approach to epilepsy healthcare in hospital settings? Could the individual characteristics found in each unique patient profile be matched to a specific IoT sensor-based device and used remotely? This is the over-arching aim of this thesis. 


\section{Chapter 3 Methodology}

\subsection{Introduction}

This chapter explains in detail the research design and methods selected in order to achieve the aim. Methods and techniques adopted to extract the data, create the ontology, perform Kmeans Clustering are discussed. Figure 3.1 shows a framework of the research design used for this thesis. The objectives (OBJ1-OBJ6) are also indicated on the 'Research Design Framework'.

For each stage of this study the various methods tools and techniques are discussed in detail in this chapter. The first stages: I \& II comprise the theoretical analysis of the principles associated with the development of ontology languages. The I. Preliminary Stage' focuses on obtaining initial knowledge of the domain by looking at current ontologies in use, consulting experts and contemplating parts of the domain by drawing initial sketches. The II Ontology Stage begins by defining the hierarchy and extracting classes, properties and relationships then implements and evaluates the ontology. The 'III. Clustering Stage' was the next logical step as data has formerly been prepared: here clustering techniques are analysed, and the chosen technique is used to perform the Cluster Analysis and evaluate the results. The last stage is the 'IV. PMP Framework and Testing Stage': here the most appropriate devices are surveyed for defined criteria required and testing on the patients takes place. The framework is also developed at this stage and finally put to test in simulated epilepsy scenarios. There is a considerable ethical scrutiny process adhered to for the testing to take place, this is undertaken at various phases throughout the study.

\subsection{Research Design}

In selecting appropriate research methods several factors were considered: the research question, ethics, and time are all major considerations. This sequential approach to research for this study involves the use of mixed research methods to achieve the aims and objectives. Mixed methods research is found to be one of the three major research paradigms including quantitative and qualitative research [98].

This study uses a sequential strategy which incorporates the mixed methods mentioned above. This is represented in the 'research design framework' seen in Figure 3.1. The research approach model was influenced by earlier findings in the literature during analysis of methodologies for ontology development whereby the generic steps and variations that are involved in the development of an ontology in a heterogeneous domain such as controlled vocabulary is key to ontology development. 


\subsubsection{Research Approach for Ontology}

This research approach was selected to address the research question by taking into account the following findings from the literature (Chapter 2):

- Use a Sequential Framework. i.e. Sampalli in 2011 [95] uses a generic framework for the development of an ontology and is divided into three important phases: a specification phase, a conceptualization phase, and an implementation phase.

- Adapt Stages of a Methodology. i.e. The methodology can be modified and tailored to its purpose and even built from scratch if necessary [96].

- Use a Manual Curation Approach. i.e. Findings indicate that when extracting terminology for an ontology: it is still commonly documented that 'manual curation' is the most reliable method for ontology development. Furthermore, as the information to be captured in this research depended critically on a combination of the domain and specific focus on capturing key terms for the domain, it was therefore crucial to select an approach which was all encompassing in order to exploit this knowledge. As observed such a sequential all-encompassing methodology can be modified to suit its purposes [96].

Understanding the parameters of a given individual 'patient profile' was imperative and the chosen framework served to depict this knowledge base. Once the ontology was created then data from the ontology can be at any time extracted from the place where it will be stored in the system in RDF or OWL and subsequently published on the Web. In this way, the ontology can be used by other users and systems based on the Semantic Web. This chosen design approach for the ontology development can be visualised implemented within a broad "Research Design Framework" see Figure 3.1 


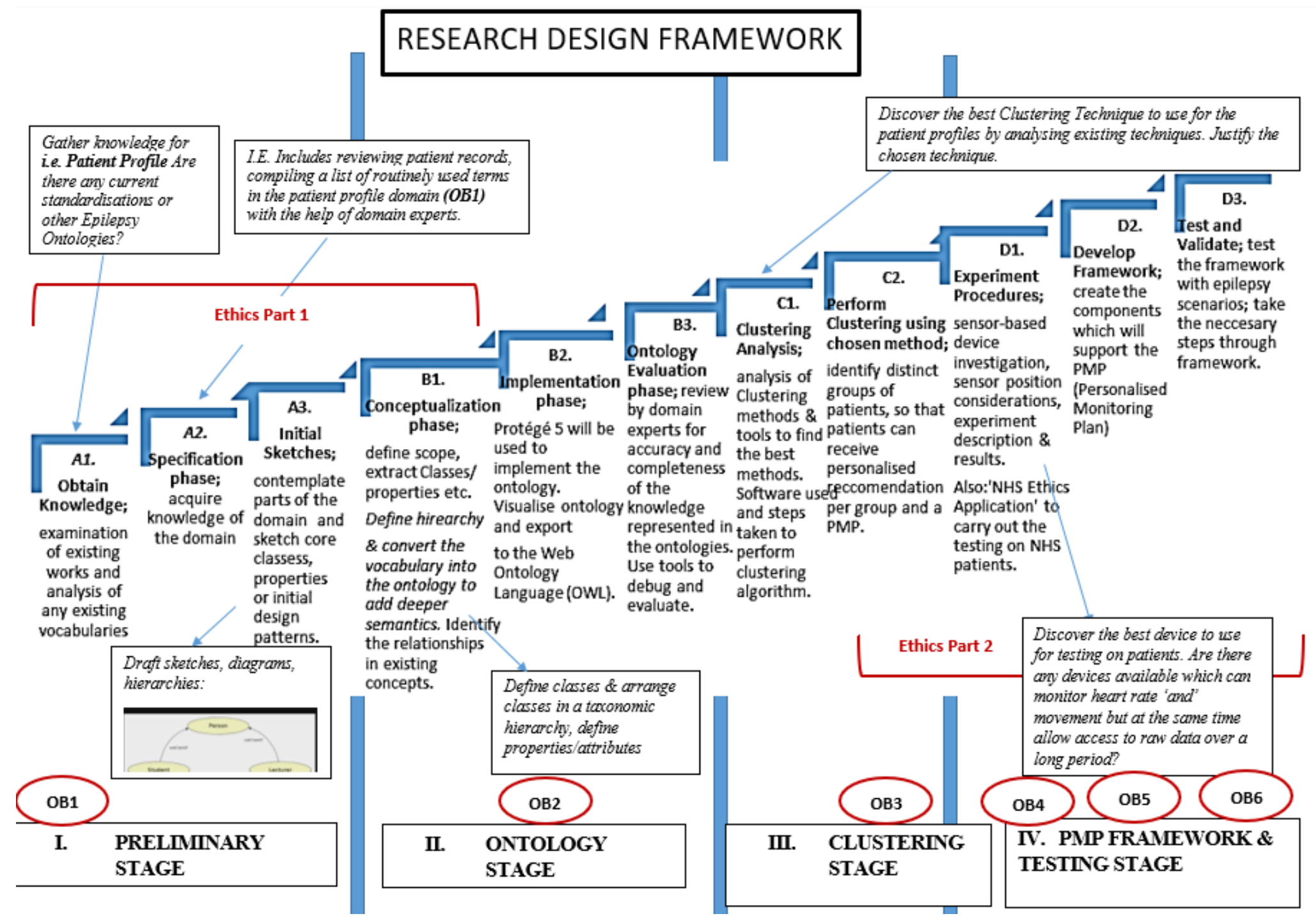

Figure 3.1. Research Design Framework 


\section{Preliminary Stage}

The methods selected for the Preliminary stage (A1:A3) are discussed below:

\section{A1. Obtain Knowledge}

As part of the knowledge discovery process 3 knowledge pools are selected:

\section{A1.1 Existing Epilepsy Ontologies}

A1.2 The ILAE (International League Against Epilepsy)

A1.3 Knowledge from clinicians.

\section{A1.1 Existing Epilepsy Ontologies}

It was significant at this stage to see if there were any other existing ontologies, and to find if these ontologies were still in use or out-dated. Even if the existing ontologies were large scale it was the intention to see if any segments of them could be useful for the creation of ESO (Epilepsy Seizure Ontology) in this study. The ontologies were studied to see if they contained any epilepsy syndromes, seizure types, classes or data elements associated with them, which could be re-used for the ESO. Another element crucial to obtaining knowledge of the domain was to understand how experts or in this instance clinicians understood seizures. Classifications and categories that could be used to inform the ESO were investigated.

\section{A1.2 The ILAE (International League Against Epilepsy)}

The initial stage of this research work investigated any current taxonomies or ontologies for seizure types/classifications. In 2017, the ILAE released a new classification of seizure types, largely based upon the existing classification formulated in 1981. To depict routinely used terms in the patient profile domain and discover if any current standardisation for epilepsy ontologies or taxonomies existed, the most recent classifications and terminologies were investigated and updated to adhere to the ILAE (International League Against Epilepsy) [99].

The ILAE strives to provide definitions for key concepts and classification schemes that will help the world epilepsy community in developing a common language to communicate effectively regarding the many facets of epilepsy. The clear terminology, based on available evidence, was used to inform the development of a language named (for the purpose of this thesis): 'PPDL' (Patient Profile Description Language). At this stage the correct medical 
terminologies were collected so that they could be embedded into the new ontology language: PPDL.

\section{A1.3 Knowledge from clinicians.}

To understand how experts or clinicians understand seizures, it was significant to gather and discover as much knowledge of the domain as conceivable.

\section{A2. Specification Phase}

In the specification phase knowledge of the domain is obtained. The two methods used in this phase are:

A2.1 Compile a list of routinely used terms

A2.2 Collect and analyse patient medical records

\section{A2.1 Categorise routinely used terms}

During this stage the intention is to further discover if there are any additional terminologies or classifications to be adhered to, this is to ensure that the ontology is reflected in the latest terminology.

\section{A2.2 Collect and analyse patient medical records}

In this specification phase knowledge of the domains in question, which were the actual 'patient profiles' were acquired, this was done by accumulating and reviewing patient medical records and most significantly collecting the anonymous patient profiles.

\section{A3. Initial Sketch and Hierarchy}

At this stage initial sketches were drawn so that initial requirements could be looked at. Parts of the domain scope were considered, and core classes, properties or initial design patterns were contemplated. These initial sketches were not intended to cover all the requirements but instead the purpose here was to look at a minimum core set of concepts. The next step was to get feedback on both the structure and the naming and associated definitions. 


\section{Ontology Stage}

The methods used for the Ontology stage (B1:B3) are discussed below:

\section{B1. Conceptualisation Phase}

This stage focuses on the collected vocabulary and uses the procedures defined below (B1.1B1.7) to convert it into the ontology to add deeper semantics.

$>\quad$ B1.1 Defining the Scope

$>\quad$ B1.2 Extract Classes \& Attributes/Properties

B1.3 Define the classes and the class hierarchy

B1.4 Define the 'object properties', 'data properties' an 'individuals'

B1.5 Domains and Ranges

B1.6 Define Data Types and Data Values

B1.7 Disjoints

\section{B1.1 Defining the Scope}

The definition of ontology goal and scope is considered the first step in the ontology creation. The scope limits the ontology, specifying what must be included and what must not [100].

It is an important step for curtailing the amount of data and concepts to be analysed [100] especially for the extent and complexity of the ESO.

In defining this common vocabulary for this specific domain or 'sector' of epilepsy information can be shared. The set of data is defined at this stage, and its structure will be available for other programs to use. Problem-solving methods, domain-independent applications, and software agents use ontologies and knowledge bases built from ontologies as data [101]. Making very clear domain assumptions [102] underlying an implementation makes it possible to change these assumptions easily if our knowledge about the domain changes. Hard-coding assumptions about the world in programming-language code makes these assumptions not only hard to find and understand but also hard to change, in particular for someone without programming expertise. In addition, explicit specifications of domain knowledge are useful for new users who must learn what terms in the domain mean [103]. Here the hierarchy is defined to depict a structured set of terms to describe the 'patient profile domain': it is used as a skeletal foundation for a knowledge base. 


\section{B1.2 Extract Classes \& Attributes/Properties}

The collected vocabulary at this stage is converted into the ontology to add deeper semantics. Prior to extracting the classes and attributes for the ESO important terms in the ontology were enumerated, similar to writing pseudocode before writing a computer program, useful lists of terms were recorded of which can be used either to make statements about or to convey information to the user of the ontology [102] i.e. What are the terms we would like to talk about? What properties do those terms have? What would we like to say about those terms? Much of this initial thinking is mined from the Preliminary Stage.

To develop the class hierarchy and define the properties of concepts the process begins by creating a few definitions of the concepts, and an initial list of key terms in the hierarchy and then continued by describing properties of these concepts and so on.

\section{B1.3 Define the classes and the class hierarchy}

A 'top-down' approach is selected to develop the class hierarchy. Several other approaches are considered [104] such as a 'bottom-up' approach and a 'combination development' method. The 'top-down' approach is used since it started with the definition of the most general concepts in the domain and subsequent specialisation of the concepts. The 'bottomup' approach is more suited to a larger ontology since it focuses on more detailed levels of grouping and used more sub-classes. The 'combination' development process which is a combination of the top-down and bottom-up approaches is again suited to larger ontologies and leans more to those using several middle level concepts. None of these three methods is inherently better than any of the others. According to Rosch the approach to take depends strongly on the personal view of the domain [105] therefore demonstrating that the curator's opinion is key to this decision.

\section{B1.4 Define the 'object properties', 'data properties' and 'individuals'}

Next the internal structure of concepts is defined. As the classes are already selected, are most of the collected remaining terms to be properties of these classes? These terms included, for example, a seizures type, symptom, frequency, patient ID etc. It becomes clear that the next 3 concepts to be identified are: 'object properties', 'data properties' and 'individuals.' Initially it is confusing as to what should a property, what should be an individual and which are object properties?

A property has a domain, which can be interpreted as the class of individuals to which this property can be applied whereas a range is the class of individuals that a property can have as its value [106]. As in OWL; object properties, are relationships between individuals and datatype properties, that is, relationships between individuals and basic types, e.g. numerical 
or string [107]. Therefore, relationships are defined: "has a": a patient "has" a seizure type a patient "has a" seizure type.

Data properties specify relationships between individuals (or instances) and data values [108]. Therefore, for the class 'seizure type' has a certain name or code, or 'patient' has “profile ID”. Whereas 'Individuals' are instances of classes, and properties may be used to relate one individual to another. For example, an individual may be described as an instance of the class Person and the property 'hasemployer' may be used to express this relationship of the individual [109].

The next step is to identify the individuals. Individuals represent the instances from the classes.

Individuals (instances) are the basic, ground level components of an ontology. Individuals are specific instances of the concepts or objects [110]. The individuals help to bring the ontology to life and visually the developer can use different individuals to 'try out' the ontology. The ESO used 30 individuals.

\section{B1.5 Domains and Ranges}

At this stage domains and ranges are defined. Properties may have a domain and a range specified. Properties link individuals from the domain to individuals from the range[111]. Simplistically, the domain and range properties are used to provide an insight into the way that the property links a domain to an object.

It is important to observe the domains and ranges as they are used as 'axioms' in reasoning, as witnessed in this example from Horrage;

"if the property hastopping has the domain set as Pizza and we then applied the hastopping property to icecream (individuals that are members of the class icecream), this would generally not result in an error. It would be used to infer that the class icecream must be a subclass of Pizza!" [111]

\section{B1.6 Define Data Types and Data Values}

According to Leipzig an ontology usually includes some kinds of entities that are classes, relations, instances, data types and data values [112]. Therefore, the next stage specifies the data types and data values of the ESO. Datatypes are important in the Semantic Web ontologies and applications, because most of which need to represent, in some way, various real world properties such as true/false, left/right or yes/no, therefore whether a string, Boolean, integer and so on. These aforementioned datatypes and data values are created and depicted in this section. Various Web ontology languages, such as RDF(S), OIL, 
DAML+OIL1 and OWL2, have witnessed the importance of datatypes in the Semantic Web. All of them support datatypes [113].

\title{
B1.7 Make classes disjoint of each other to avoid multiple inheritance
}

In preparation for the 'Implementation Phase' (using Protégé) it is important to make classes disjoint of each other to avoid multiple inheritance, so that there will only be an instance of one class.

Hierarchies in which all types have only a single parent are referred to as single inheritance hierarchies, whereas hierarchies in which types can have more than one parent are referred to as multiple inheritance hierarchies. The problem with using multiple modes of classification and with allowing multiple inheritance is that the meaning of the 'is_a' relation becomes uncertain, resulting in errors on the part of both maintainers and users of an ontology [114] and the inability to use the hierarchy for automated reasoning. While in practice it can be difficult to avoid multiple inheritance, even within a single mode of classification, multiple modes of classification (and therefore multiple meanings for is_a) should be avoided by using the corresponding specific relations (e.g. Has_location). The benefits are not only an ontology that has fewer errors, is easier to maintain, and can be used for automated reasoning, but also a reduced loss of information by using the more specific representation [115].

\section{B2. Implementation Phase}

The next stage in the process is to build the ontology with Protégé [116]. The methods selected at this phase are divided and discussed in the following sections:

\author{
B2.1 Specify IRI \\ B2.2 Implement the Ontology \\ B2.3 Publish the Ontology \\ B2.4 Visualisation Tools \\ B2.5 Exporting to Web Ontology Language (OWL)
}

\section{B2.1 Specify IRI}

An IRI is specified to begin the ontology set-up. 


\section{B2.2 Implement the Ontology}

Protégé 5 is used to implement the ontology (ESO). Protégé is an ontology development tool that is used to build and edit the ontology. The Stanford-created, Java-based program Protégé is chosen as the implementation mechanism for ESO. The Protégé software provides a development environment for the creation of an ontology through GUIs. Protégé has become a widely accepted program used for implementing ontologies into a computational environment and was chosen as a free, open-source software. Apart from Protégé there are more leading ontology editors including Ontoedit, Oiled, Chimaera. Yet Protégé has greater community support and deploys visual tools which lend itself appropriately to academia and for the purposes of ESO.

\section{B2.3 Publish the Ontology}

The ontology is published online so that it can be accessible for Evaluation Tool use later.

\section{B2.4 Visualisation Tools}

Protégé provides excellent extensions for ontology visualisation. In order to comprehend the structure of the ESO, graph visualisation was used. Ontograph provided useful support for interactively navigating the relationships of the ESO ontology; there are various layouts that Ontograph supports which automatically organise the structure of the ontology. It also supports different relationships and each subclass, individual, domain/range object properties, and equivalence. The developer can filter relationships and node types which aid to create desirable views [117]. Findings also identify that there is a high demand for further assessment and analysis of the ontology and these tools which graphically visualise ontologies are being used to aid this [118] therefore the OWLviz plugin is used in addition, to allow the asserted and inferred classification hierarchies to be visualised.

\section{B2.5 Exporting to Web Ontology Language (OWL)}

The next step is to export the ontology into the Web Ontology Language (OWL) and to transform it to a semantic ontology. The Web Ontology Language OWL extends RDF and RDFS. Its primary aim is to bring the expressive and reasoning power of description logic to the semantic web [109]. The semantic web is a set of standards and best practices for sharing data over the web for use by applications not for human beings. RDF is the standard for semantic web (not a language but a data model). OWL builds on RDF; the reason OWL is used is it lets us store bits of meaning so we can get more out of our data. 
Protégé fully supports the latest OWL 2 Web Ontology Language and RDF specifications from the World Wide Web Consortium [119]. The OWL Plugin is a Semantic Web extension of the Protégé ontology development platform. The OWL Plugin can be used to edit ontologies in the Web Ontology Language (OWL), to access description logic reasoners, and to acquire instances for semantic mark-up.

Embracing the semantic web is an important consideration for the future of PPDL, whereby web languages used by the semantic web can become IoT enabled and better enable objects, devices and people to work in co-operation [87]. The initiative by Tim Berners Lees' semantic web can link knowledge-based systems such as PPDL.

\title{
B3. Ontology Evaluation Phase
}

In this phase the methods selected to debug the ontology and remove errors are demonstrated. Finally, to evaluate the ontology a review by domain experts for accuracy and completeness of the knowledge represented in the ontologies is undertaken.

The steps are divided and discussed in the following sections:

\author{
B3.1 Reasoners in Protégé \\ B3.2 Protégé Debugger \\ B3.3 Evaluation Tools \\ B3.4 Review by Experts
}

\section{B3.1 Reasoners in Protégé}

To ensure the quality of the ESO ontology, and to deal with any inconsistency and uncertainty for use in other applications reasoners in Protégé are used. The quality and correctness of ontologies play a vital role in semantic representation and knowledge sharing [120]. DL reasoners verify whether there are any logical contradictions in the ontology axioms [121].

An inconsistent ontology means that an error or a conflict exist in an ontology, as a result some concepts in the ontology cannot be interpreted correctly. The inconsistency will result in false semantic understanding and knowledge representation. An uncertain ontology means that the correctness of the ontology is probabilistic. Ontology reasoning reduces the redundancy of information in knowledge base and finds the conflicts in knowledge content [121].

Reasoner is a software that is used to derive new facts from the existing ontologies. Some of the popular reasoners developed in the last few years are: Pellet, RACER, FACT++, 
Snorocket, Hermit, and others. A reasoner is a program that infers logical consequences from a set of explicitly asserted facts or axioms and typically provides automated support for reasoning tasks such as classification, debugging and querying [122].

Protégé 5 has two reasoner options, FACT++, and Hermit 1.3.8. FACT ++. FACT ++ is the reasoner chosen for the ESO, even though hermit is a reasoner based on Description Logic (DL) it is more suited to larger and more complex ontologies [123].

An updated version of fact is FACT ++. This reasoner uses the same algorithm as in fact, but with a different internal structure. It is implemented in $\mathrm{C}++$. The first version of the FACT ++ was only supporting the reasoning in SHOIQ, OWLDL. However, the latest version of FACT ++ supports OWL and is based on the description logic SROIQ. FACT ++ implements a tableau-based decision procedure for general tboxes and incomplete support for aboxes [124]. The quality and correctness of ontologies plays vital role in semantic representation and knowledge sharing [120].

After an ontology is developed, it is used, reused, and related to other ontologies, and also needs to be maintained. These tasks may be easier when an ontology is designed with these tasks in mind. For example, building ontology on a shared upper ontology and using a modular design usually means easier use and maintenance.

\section{B3.2 Protégé Debugger}

The debugger in Protégé is also used to check if ESO is coherent and consistent.

\section{B3.3 Evaluation Tools}

There are numerous theoretical models and metrics for evaluating an ontology, but still there is a problem for evaluating ontologies as some anomalies can appear in developed ontologies since ontologists should handle diverse difficulties [125]. Therefore, in any ontology development project it is vital to perform the ontology evaluation activity since this activity checks the technical quality of an ontology against a frame of reference [126].

The vision of the Semantic Web originally proposed by Berners-Lee et al. [126] has promoted the continued growth of dataset published as well as the publication of ontologies used to model and enrich semantically these data. In this scenario, as important of the quality of the data published should be the quality and validation of the ontologies bringing semantic to such data. These ontologies should be published according to LD principles, but they also must be evaluated at different stages, both during their development and their publication. Thus, the evaluation of those ontologies has become a significant challenge. Ontologies developed following a particular methodology (e.g., NeOn Methodology [127] or 
Ontoknowledge [128] have normally higher quality than those built without using methodological guidelines. However, to apply methodologies for building ontologies do not guarantee to have final products completely free of errors. In order to produce a list of evaluation results, the Ontology Pitfall Scanner [129] takes as its input the ontology to be analysed. The system is accessible to humans by means of a web user interface and to machines throughout a web restful service that will be detailed in Section 3 and Section 4 respectively. The input ontology could be entered by its URI or the OWL code 2, which describes the ontology to be analysed.

Once the ontology is parsed using the Jena API3, the Pitfall Scanner module inspects the declared ontology looking for pitfalls among those available in the catalogue. During this scanning phase, the ontology elements prone to potential errors are detected, whereas some modelling suggestions are generated by the Suggestion Scanner module. Then, the evaluation results are provided including the list of pitfalls detected, if any, and the ontology elements affected, as well as explanations describing the findings. The system allows not only analysing all the automated pitfalls, but also choosing specific pitfalls or predefined groups according to the pitfall classification. Finally, a conformance badge is provided to the user together with the HTML code for including it within the ontology documentation website [129].

\section{B3.4 Review by Experts}

Review by domain experts for accuracy and completeness of the knowledge represented in the ontologies. The experts will be asked to complete an evaluation form and answer evaluation questions to clarify any ambiguities throughout the development of the ontology.

\section{Clustering Stage}

The methods used for the Clustering stage (C1:C2) are discussed below:

\section{C1. Clustering Analysis}

Prior to performing clustering, this stage carries out an analysis on clustering tools. Discussed in section $\mathrm{C} 1.1$.

\section{C1.1 Clustering Analysis Overview}

This section investigates the concept of clustering in general. Beginning by looking at its theory and background it further discusses its use in society and how clustering essentially models data. It was important to see what role clustering plays in other applications and if it 
has achieved any success. Finally, it was imperative to see how it will be applicable/beneficial to this research.

By looking at the concept of clustering, it was valuable to see what it tries to achieve, this was done and discussed by looking at examples. The techniques and algorithms available for clustering were analysed and discussed with the goal of determining the best approach to take for this study.

Accuracy is important in the clustering environment and factors which affect this are flexibility and scalability. This is considered when deciding upon a partitioning or hierarchical type of clustering, this is discussed in this section. Euclidean Distance Calculation (EDC) used to achieve efficiency was also discussed in this section.

After analysing and discussing clustering analysis and techniques finally the chosen clustering method was selected which will be described in detail in the 'Clustering' Chapter 5.

\section{C2. Perform Clustering}

The next stage outlines the methods used to performing Clustering with the chosen clustering method. Dataset preparation and pre-processing methods are used and discussed at this stage. Further Analysis on the dataset used in the 'Ontology Stage' is performed to ensure it is ready for OBJ.3

Then follows 'Data Cleaning' whereby categorical attributes are converted to numeric values to be used for K-means. This stage then uses the Pre-Processing tool in WEKA to [130] remove any noise or redundant data.

There are many processes addressed and carried out to perform K-means Clustering on the pre-processed dataset. When performing the K-means algorithm in WEKA properties need to be measured and moulded to fit the needs of the outcome. These properties are, 'Percentage Split', 'Choosing the Seed' and 'Choosing the Optimal Number of Clusters'. The aforementioned methods are carried out and discussed in Chapter 5 'Clustering'. Once the clustering is performed the K-means Results are evaluated and findings are discoursed, together with a discussion about how 'Sum of Squared Errors' is used to validate the cluster results. Finally, tools in WEKA are used for visualising the results, which provide a further analysis of the results.

Sections are divided accordingly into the following sections below and discussed in detail in Chapter 5.

C2.1 Dataset Preparation \& Pre-processing

C2.2 Data Cleaning 
C2.3 Clustering Considerations

C2.4 Percentage Split

C2.5 Choosing the Seed

C2.6 Choosing the optimal Number of Clusters

C2.7 Evaluating K-means Results

C2.8 Sum of Squared Errors

C2.9 Visualising Results

\title{
IV. PMP Framework \& Testing stage
}

The methods used for the PMP \& Testing stage (D1:D3) are discussed below:

\section{D1. Experiment Procedures}

This section discussed the experiment procedures and carried out methods such as an analysis of possible IoT sensor-based devices and positions, experiment description and objectives, together with pilot study methods and finally the experimental results.

Central to the study was the investigation to discover possible IoT sensor-based devices, in order to identify and obtain the IoT sensor-based device information methods are proposed here for collection and analysis.

Additionally, the retrieval of this data from the devices also requires the researcher to understand and utilise techniques to analyse and visualise information collected from the device. This characteristic is also sought, and all are discussed in Chapter 6.

The methods selected are outlined below. This mixed methods approach is selected because often a combination of techniques that gathers both quantitative and qualitative information will yield the most comprehensive results [131]. Furthermore, using a combination of methods, (see list below) whilst time consuming, has the advantage of gaining a comprehensive insight ensuring a full range and depth [131]:

\author{
Literature Review \\ Forum Discussions \\ Data Extraction Tests \\ Experiments with Apps \\ Observations and Surveys
}




\section{Information Gathering:}

○ Discussions with Commercial companies

- Contacting Researchers at other Universities

- $\quad$ Exploring Data

- $\quad$ Exploring all capable and available sensors

- Discovering techniques to visualise extracted data

Correct sensors had to be discovered in order to analyse and discover if there is any change in a patients behaviour through assessing an IoT sensor-based device performance during a seizure: hence to see if the patients known time of seizure matched the sensor behavior on the device.

This section also analysed 'sensor positions' i.e. Can an individual patients profile indicate which part of the body the sensor should be positioned?

This experiment focused on the theory (study hypothesis) of the 'personalised approach' to see if it worked in practice, hence: could the individual epilepsy patient profile make a difference in which device to wear? The experiment objectives, eligibility criteria and data collection and analysis are discussed here, (see Chapter 6).

These experiments will be carried out by consenting NHS patients wearing the IoT sensorbased device containing a custom-made APP which will ascertain the time of seizure from the accelerometer and heart-rate sensor.

To carry out testing upon hospital patients, a vigorous NHS application process was undertaken using 'The Integrated Research Application System' (IRAS). The IRAS is a single system for applying for the permissions and approvals for health and social care / community care research in the UK. The methodology for this study and the protocol is discussed later in Chapter 6.

Once the IoT Sensor-based device was chosen a pilot study was undertaken. Preliminary Experiments with the selected sensor-based device are performed, and simulated data from the sensors is collected.

Results and findings from experiments on epilepsy patients and volunteers are discussed and analysed in this section.

\section{D2. Develop PMP Framework}

This section discusses the development of the PMP framework. The framework was designed with components which will support the PMP (Personalised Monitoring Plan), this is 
presented in Chapter 7 . The components are discussed at this stage, the two main features are 'Personalisation' and 'Monitoring and Management'. At this stage only the 'Personalisation' components are implemented. It is not intention of this thesis to implement a fully operational system but instead a sequential framework which represents components of the system. To produce the PMP framework this research has chosen a sequential method and offers a conceptual framework for understanding and approaching the decisions and obstacles that arise in monitoring epilepsy. As observed by Mcgaghie Et Al: when presenting the particular research question the conceptual framework sets the scene that drives the investigation being reported based on the problem described [132].

\section{D3. Testing and Validation}

This section discussed the testing and validation of the PMP framework. Here the PMP frameworks' personalised approach capabilities are tested so that the research hypothesis can be validated.

In Chapter 7 the PMP framework is tested in two epilepsy scenarios through a Use Case Evaluation ( see 7.6 Use Case Evaluation) and the results determine how effective the developed framework can be used as a tool for recommending the IoT device to an epilepsy individual patient. Therefore, significant evidence will be presented from the epilepsy scenarios and ensure that it is a dependable and useful tool.

The testing will be conducted by healthcare professionals in simulated epilepsy scenarios whereby random anonymous individual patient profiles will be input to the PMP framework.

\subsection{Summary}

This chapter explained in detail the research design and methods selected to carry out the research this thesis. The sequential 'Research Design Framework' shown in Figure 3.1. Research Design Framework) demonstrated a novel way to approach the research development in this thesis. 


\section{Chapter 4 Ontology for PPDL}

\subsection{Introduction}

This chapter presents the steps taken to gather the knowledge to inform the outcome which is the PPDL (Patient Profile Description Language). In gathering this knowledge, the ESO 'Epilepsy Seizure Ontology' is developed.

To begin there is the knowledge discovery process with a clear focus on the dependable attributes for a 'patient profile' showing how they are discovered and fed into the ESO to develop the PPDL. This chapter discusses the implementation of the ontology and presents the authentication of the ESO in line with the present vocabularies used in current hospital settings demonstrating how they will be useful to create a PMP (Personalised Monitoring Plan).

Focusing upon 'I. Preliminary Stage' and 'II. 'Ontology Stage' of the Research Design Methodology, (see Figure 4.1) presented in Chapter 3, stages A1:B3 are discussed. Beginning with where the knowledge is obtained, right through to the Ontology Evaluation this chapter will demonstrate (using the stages of Research Design) the outcomes at each stage and explain their respective rationales.

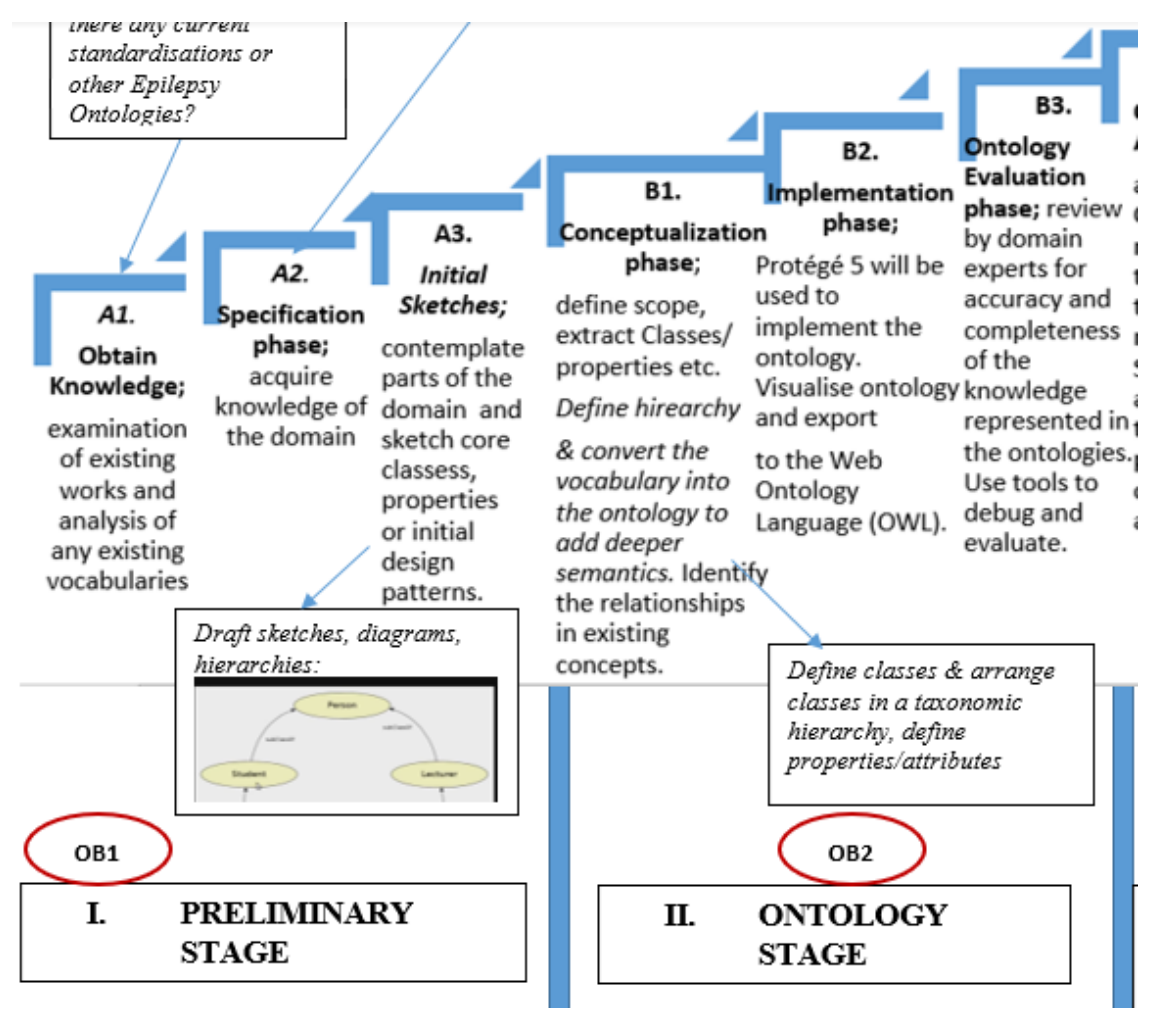

Figure 4.1. Preliminary and Ontology Stage 


\subsection{Preliminary Stage (I)}

The Preliminary stage consists of three activities (A1-A3)

\section{A1. Obtain Knowledge}

As part of the knowledge discovery process 3 knowledge pools are selected:

A1.1 Existing Epilepsy Ontologies

A1.2 The ILAE (International League Against Epilepsy)

A1.3 Knowledge from clinicians.

\section{A1.1 Existing Epilepsy Ontologies}

At present, there are more than 300 biomedical ontologies listed at the NIH-funded National Center for Biomedical Ontologies (NCBO) [133], at the time of writing this thesis only 3 epilepsy ontologies are found in this database, see Figure 4.2. Epilepsy Ontologies [133]. These 3 existing epilepsy ontologies are large scale. One widespread and prevalent ontology is EPSO (The Epilepsy and Seizure Ontology) this being the largest with 1357 classes. EPSO is the most all-encompassing ontology, whereas another epilepsy ontology; 'EPILONT', although based on the diagnosis proposed by the International League Against Epilepsy (ILAE) is focused specifically upon Eletroencefalogia which is apparatus for epilepsy. ESSO (2015) a more recent ontology contains epilepsy syndromes, seizure types, and data elements associated with them, this is useful, and classes can be re-used for the ESO but it is not updated to ILAE 2017. As identified by Berg et al; since epilepsy is known to have inherent complexity and a diverse stakeholder community this in turn affects the task of the classification of epilepsy, making it a complex and controversial undertaking" [134].

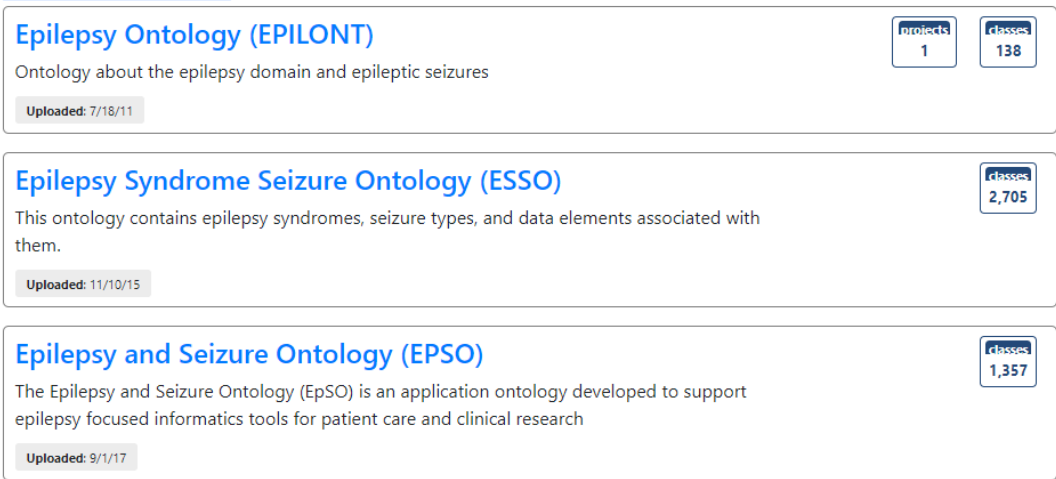

Figure 4.2. Epilepsy Ontologies 


\section{A1.2 The ILAE (International League Against Epilepsy)}

The most recent classifications and terminologies are followed from the ILAE . The clear terminology, based on available evidence, is used to inform the development of the PPDL (Patient Profile Description Language). The ILAE classifications are large scale but nevertheless the correct medical terminologies are adhered to by updating the old terms to the new terms and noting and abbreviations, for each seizure type, symptom and sign. These are embedded into the ontology. A snapshot of this process is shown below Table 4.1. ILAE Classification's.

Table 4.1. ILAE Classification's

\begin{tabular}{|c|c|c|}
\hline \multicolumn{3}{|c|}{ SEIZURE TYPES } \\
\hline Old Term & New Term & $\begin{array}{l}\text { Available } \\
\text { Abbreviation }\end{array}$ \\
\hline $\begin{array}{l}\text { Generalised } \\
\text { Tonic Clonic }\end{array}$ & $\begin{array}{l}\text { Generalised } \\
\text { Tonic Clonic }\end{array}$ & GTCS \\
\hline $\begin{array}{l}\text { Focal (lots of } \\
\text { shaking) }\end{array}$ & $\begin{array}{l}\text { Focal Motor } \\
\text { Seizure }\end{array}$ & FMS \\
\hline Secondary & $\begin{array}{l}\text { Focal to } \\
\text { bilateral tonic- } \\
\text { clonic seizure }\end{array}$ & FBTCS \\
\hline $\begin{array}{l}\text { Focal Motor } \\
\text { Seizure }\end{array}$ & $\begin{array}{l}\text { Focal Motor } \\
\text { Seizure }\end{array}$ & FMS \\
\hline Drop seizure & $\begin{array}{l}\text { Generalized } \\
\text { Atonic }\end{array}$ & GA \\
\hline Absence & $\begin{array}{l}\text { Non-Motor } \\
\text { Absence }\end{array}$ & NMA \\
\hline Visual Auras & $\begin{array}{l}\text { Focal sensory } \\
\text { seizure }\end{array}$ & FSS \\
\hline
\end{tabular}




\section{A1.3 Knowledge from clinicians.}

Another element crucial to obtaining knowledge of the domain is to understand how experts or in this instance clinicians understand seizures. It is discovered within literature [99] that clinicians generally classify seizures by "Symptoms", "Signs" and "Seizure Type". These 3 classifications are used to inform the PPDL

It is also found that the current practices (within the hospital settings) collect seizure type information upon patient appointment and during monitoring. Each patient has their own 'medical record' which is completed and updated during these times. Discussions with clinicians revealed:

- There is no current catalogue of any patients attributes i.e. A database which lists symptoms such as, 'metallic taste' or 'déjà vu' or 'finger rubbing'. Likewise, there is no specific list for 'Seizure Types'. Instead seizure type information is presented sporadically within the patient records i.e. Within descriptive paragraphs. Indeed all 3 classifications are mentioned, together with their associated attributes but these classifications are not easy to extract.

- In addition to the 3 classifications other information that is revealed by clinicians during discussion is:

- The way in which a symptom and sign is interpreted during practice: a symptom is something the patient reports. A sign is what the doctor observes. For example, an aura (the feeling the patient has before a seizure) could be a symptom of a seizure (as the patient is reporting it). However, if the patient is seen stiff and shaking that would be a sign. The combination of symptoms and signs results in the diagnosis of epilepsy.

- Patients are identified in 2 categories: 'Observed Patients' and those that are 'Appointment Patients' and self-monitored (seen every 3/6 months). The 'Observed Patients' are only the patients who are having frequent episodes considered more than 2-3 per week. The observed patients are monitored by video telemetry \& EEG and are in hospital for 5 days.

- When analysing the observed data from the video telemetry \& EEG there are no techniques used to learn from the data, the lab technicians basically look and can identify with the naked eye when a seizure occurs.

In summary of these findings the overall observations reveal that there is indeed a gap highlighting a need for patient seizure type information to be organized and categorised. The 
3 classifications found in literature are strictly used within hospital settings, and the interpretation of 'signs' and 'symptoms' has also been understood. Interestingly patients with frequent episodes are considered 2-3 per week and need to be observed by the hospital. Lastly the datasets from each patient when being monitored are used 'only' to detect the seizure in real-time and the data does not appear to be used for any analysis after the seizure event, illustrating an opportunity for utilising this data in the future.

\section{A2. Specification Phase}

In the 'specification phase' knowledge of the domain is obtained. The 2 goals in this phase are:

A2.1 Compile a list of routinely used terms

A2.2 Collect and analyse patient medical records

\section{A2.1 Compile a list of routinely used terms}

During this stage the intention is to further discover if there are any additional terminologies or classifications to be adhered to, this is to ensure that the ontology is reflected in the latest terminology. It is discovered in 2017 that in the study by Fisher et al [99] a way of categorising the main routinely used terms related to seizures, is identified see Figure 4.3. Categorising Routinely used Terms.

These additional categorisations aid to individualise the patient profile and are embedded in the ontology. 


\begin{tabular}{|c|}
\hline $\begin{array}{l}\text { Cognitive } \\
\text { acalculia } \\
\text { aphasia } \\
\text { attention impairment } \\
\text { déjà vu or jamais vu } \\
\text { dissociation } \\
\text { dysphasia } \\
\text { hallucinations } \\
\text { illusions } \\
\text { memory impairment } \\
\text { neglect } \\
\text { forced thinking } \\
\text { responsiveness impairment }\end{array}$ \\
\hline $\begin{array}{l}\text { Emotional or affective } \\
\text { agitation } \\
\text { anger } \\
\text { anxiety } \\
\text { crying (dacrystic) } \\
\text { fear } \\
\text { laughing (gelastic) } \\
\text { paranoia } \\
\text { pleasure }\end{array}$ \\
\hline $\begin{array}{l}\text { Autonomic } \\
\text { asystole } \\
\text { bradycardia } \\
\text { erection } \\
\text { flushing } \\
\text { gastrointestinal } \\
\text { hyper/hypoventilation } \\
\text { nausea or vomiting } \\
\text { pallor } \\
\text { palpitations } \\
\text { piloerection } \\
\text { respiratory changes } \\
\text { tachycardia }\end{array}$ \\
\hline
\end{tabular}

\begin{tabular}{|c|}
\hline $\begin{array}{l}\text { Automatisms } \\
\text { aggression } \\
\text { eye-blinking } \\
\text { head-nodding } \\
\text { manual } \\
\text { oral-facial } \\
\text { pedaling } \\
\text { pelvic thrusting } \\
\text { perseveration } \\
\text { running (cursive) } \\
\text { sexual } \\
\text { undressing } \\
\text { vocalization/speech } \\
\text { walking }\end{array}$ \\
\hline $\begin{array}{l}\text { Motor } \\
\text { dysarthria } \\
\text { dystonic } \\
\text { fencer's posture (figure-4) } \\
\text { incoordination } \\
\text { Jacksonian } \\
\text { paralysis } \\
\text { paresis } \\
\text { versive }\end{array}$ \\
\hline $\begin{array}{l}\text { Sensory } \\
\text { auditory } \\
\text { gustatory } \\
\text { hot-cold sensations } \\
\text { olfactory } \\
\text { somatosensory } \\
\text { vestibular } \\
\text { visual }\end{array}$ \\
\hline $\begin{array}{l}\text { Laterality } \\
\text { left } \\
\text { right } \\
\text { bilateral }\end{array}$ \\
\hline
\end{tabular}

Figure 4.3. Categorising Routinely used Terms

\section{A2.2 Collect and analyse patient medical records}

100 anonymous patient medical records are collected. Once the data is gathered it is analysed to discover if values for each of the attributes are different for each patient, and to address the research question derived from RQ $1 \mathrm{~b}$. "How can an understanding of typical seizure patient profiles be achieved?" To do this the key considerations during this process, are listed below and then a discussion follows:

- $\quad$ 'Are there any further categories to note and embed?'

- $\quad$ 'Are there any commonalities found between seizure type patients?'

- $\quad$ 'What are the main symptoms discovered?' and 'are the seizures diurnal or nocturnal?

Upon analysing the 100 patient medical records further categories are observed and embedded to support individualising the patient profile, i.e. 'diurnal (daytime) or nocturnal (nigh time)' and 'frequency' (to ascertain how often the seizures occur).

The data collected demonstrates that values for each of the attributes are different for each patient, a snapshot of this data is shown in Table.4.2, 'Personalisation Measures'. This table presents a diverse selection of patient profiles to demonstrate personalisation measures. The 
patient medical dataset displayed in this table was originally derived from unstructured data found in patient medical records which had no pre-defined organisation. Therefore, to make it easier to analyse potential personalisation measures the data was organised into categories under the headings: profile id, seizure type, alert for patient, typical actions during seizure, occurs from sleep and frequency. These initial categories discovered from the patient records were considered one of the crucial steps in determining the individual patient. The colouring scheme used in Table 4.2 is referred to in the next paragraphs and further emphasised in a key provided at the end of the table.

Not one patient is the same as the other; every patient's values are unique. Based upon the first 50 records, there are 13/50 patient profiles whose seizures are one or more daily, this is 'high frequency', indicated in red in Table 4.2. Personalisation Measure's.

Commonalities found between these seizure type patients are that the patients mainly have the 'seizure type' known as GTC (General Tonic Clonic Seizures), indicated in purple, but the seizure occurrence is either diurnal (daytime) or nocturnal (night time), yet often 'both' (in yellow) and the 'signs' are anything from stress, metallic taste, tingling hands, confused, dizzy or none at all.

One of the main symptoms found for "Symptoms" during the seizure is shaking (or other times described as jerking or whole body shaking), known as 'clonus', shown in green. Interestingly only patient out of 100 had a 'Gelastic' seizure, (in blue). 


\begin{tabular}{|c|c|c|c|c|c|}
\hline Profile & Seizure Type & Alert for patient & Typical Actions During seizure & Occurs from Sleep & Frequency \\
\hline $\begin{array}{l}\text { Profile } 1 \\
\text { (384362) }\end{array}$ & $\begin{array}{l}\text { - Focal lots of } \\
\text { generalised } \\
\text { shaking events }\end{array}$ & $\begin{array}{l}\text { Panic attack } \\
\text { Symptoms } \\
\text { i.e. heart-rate } \\
\text { palpitations, unable to } \\
\text { catch breadth }\end{array}$ & $\begin{array}{l}\text { Full jerking of body or } \\
\text { Can just be arms or legs (no tongue } \\
\text { biting) } \\
\text { Hip and leg twitching }\end{array}$ & $\begin{array}{l}\text { Yes (both: day \& } \\
\text { night) }\end{array}$ & $\begin{array}{l}\text { Up to } 6 \text { weeks without but then } \\
\text { can cluster }\end{array}$ \\
\hline $\begin{array}{l}\text { Profile } 2 \\
\text { (132266) }\end{array}$ & $\begin{array}{ll}\text { - } & C P S \\
\text { - } & \text { Secondary } \\
\text { generalised } \\
\text { seizures }\end{array}$ & $\begin{array}{l}\text { Occasional warning: } \\
\text { odd feeling in head } \\
\text { then appears vacant }\end{array}$ & $\begin{array}{l}\text { Possible lip smacking occasionally } \\
\text { LOC, convulsions. (during recording: } \\
3 \text { times the patient raised the right } \\
\text { arm, \& shaking movements were } \\
\text { observed with some repetitive rubbing } \\
\text { of fingers) }\end{array}$ & $\begin{array}{l}\text { Yes (both: day \& } \\
\text { night) }\end{array}$ & 1-2 per month \\
\hline $\begin{array}{l}\text { Profile } 3 \\
\text { (164396) }\end{array}$ & $\begin{array}{l}\text { - Partial seizure } \\
\text { - } \text { GTC }\end{array}$ & $\begin{array}{l}\text { Suddenly switches off: } \\
\text { does not seem real }\end{array}$ & Partial seizure then GTC & & $\begin{array}{l}1 \text { every } 2 \text { days } \\
\text { (can be up to } 5 \text { per day) }\end{array}$ \\
\hline
\end{tabular}




\begin{tabular}{|c|c|c|c|c|c|}
\hline $\begin{array}{l}\text { Profile } 4 \\
\text { (38966) }\end{array}$ & $\begin{array}{l}\text { - Blank Spells } \\
\text { (Absences) } \\
\text { - GTC }\end{array}$ & $\begin{array}{l}\text { Metallic Taste for } \\
\text { absences } \\
\text { No warning for GTC }\end{array}$ & $\begin{array}{l}\text { Body Stiffening \& shakes, foaming at } \\
\text { mouth, tongue biting, }\end{array}$ & $\begin{array}{l}\text { Yes (both: day \& } \\
\text { night) }\end{array}$ & $\begin{array}{l}1 \text { every } 2 \text { days } \\
\text { (can be up to } 5 \text { per day) }\end{array}$ \\
\hline $\begin{array}{l}\text { Profile } 5 \\
\text { (118291) }\end{array}$ & $\begin{array}{l}\text { - } \quad \text { GTC } \\
\text { - } \quad \text { Visual Auras }\end{array}$ & $\begin{array}{l}\text { Visual Disturbance: } \\
\text { flickering coloured } \\
\text { lights in right eye that } \\
\text { spread to left then loss } \\
\text { of vision }\end{array}$ & $\begin{array}{l}\text { GTC, tongue biting, occ. Urinary } \\
\text { incontinence }\end{array}$ & $\begin{array}{l}\text { Yes (both: day \& } \\
\text { night) }\end{array}$ & $\begin{array}{l}\text { Weekly Aura } \\
\text { GTC controlled by drugs }\end{array}$ \\
\hline Profile 20 & 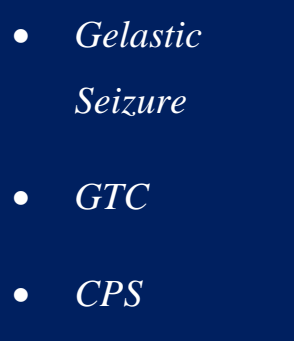 & $\begin{array}{l}\text { Aura of dizziness, } \\
\text { fuzzy head, raising } \\
\text { sensation }\end{array}$ & $\begin{array}{l}\text { Bursts of Seizure, LOC, Confused } \\
\text { behaviour, tongue Biting }\end{array}$ & Both Day and night & $\begin{array}{l}\text { Daily } \\
\text { Gelastic (10 a day) } \\
\text { GTC: } 3 \text { per month } \\
\text { CPS: } 1 \text { per week }\end{array}$ \\
\hline Profile 21 & - $\quad G T C$ & $\begin{array}{l}\text { When stressed, more } \\
\text { frequent }\end{array}$ & $\begin{array}{l}\text { Jerking of limbs, occasional } \\
\text { incontinence }\end{array}$ & Night & 1 or 2 a week \\
\hline Profile 22 & - $\quad G T C$ & Déjà vu, head rush & $\begin{array}{l}\text { Shaking, tongue biting, incontinence, } \\
\text { confused }\end{array}$ & Daytime & $\begin{array}{l}\text { Every } 4 \text { to } 5 \text { days. Up to } 10 \text { per } \\
\text { day end of Menstrual Cycle }\end{array}$ \\
\hline
\end{tabular}




\begin{tabular}{|c|c|c|c|c|c|}
\hline Profile 23 & $\begin{array}{l}\text { - } \quad \text { GTC } \\
\text { - Complex } \\
\text { Partial Seizure }\end{array}$ & No warning & $\begin{array}{l}\text { Appears vacant, picking at cloths, lip } \\
\text { smacking }\end{array}$ & Night-time & 2-3 per month \\
\hline Profile 24 & $\begin{array}{l}\text { - Absences } \\
\text { - } G T C\end{array}$ & No warning & $\begin{array}{l}\text { LOC, shaking, } \\
\text { Tongue biting }\end{array}$ & Daytime & $\begin{array}{l}\text { Absences: } 1 \text { per day } \\
\text { GTC: Every 2-3 months }\end{array}$ \\
\hline Profile 25 & - Drop Seizure & No warning & $\begin{array}{l}\text { LOC, falling asleep without warning, } \\
\text { twitching }\end{array}$ & Daytime & 3-4 times a week \\
\hline $\begin{array}{l}\text { Profile } 6 \\
\text { (55794) }\end{array}$ & - $\quad G T C$ & $\begin{array}{l}\text { No warning then } \\
\text { Collapse, LOC }\end{array}$ & $\begin{array}{l}\text { Collapse, LOC, } \\
\text { During GTCS: Intermittent shaking of } \\
\text { right arm (lasting over an hour) } \\
\text { Many similar events of right hand/arm } \\
\text { shaking }\end{array}$ & $\begin{array}{l}\text { Yes (both: day \& } \\
\text { night) }\end{array}$ & 2-3 times per week \\
\hline & $K E Y$ & High Frequency (daily) & Both Day \& Night & Gelastic Seizure & Clonus \\
\hline
\end{tabular}




\section{A3. Initial Sketch and Hierarchy}

The initial sketches for the ontology facilitated initial requirements and at this juncture parts of domain are contemplated. When developing an ontology next step is to identify the core classes of the domain, this initial step addresses organisation and introduces structure to the terms[135]. The core classes identified are the 'Patient' and 'Seizure', additionally the associated entities considered significant are 'Seizure Frequency' and 'Seizure Indicator'. The 'type' of seizure is also important i.e. 'Focal', 'GTA', 'Partial', it is envisaged that these are the 'properties' of the core classes. Sketching some initial diagrams helps to begin the thought process in terms of 'hierarchy' and the driving questions are:

- How can each individual patient profile (1-100) be represented?

- Should the seizure indicator be a sub-class of seizure or patient?

- How can the frequency of the seizure be categorised?

It is apparent during this stage how the ontology development is going to be an iterative process; nouns and verbs from the knowledge source are used to sketch an concept of the overall structure and it is envisaged that later stages will go through multiple iterations to refine the structure, [136] see Figure 4.4. Initial Sketch \& Hierarchy.

(Where the figure demonstrates the rough idea that a seizure 'may' occur from 'sleep/not sleep' simply demonstrates 'either/or' and for clarification purposes there is no foreseen plan within the scope of this thesis at this stage to capture stochastic information.)

These initial sketches are not intended to cover all the requirements but instead the purpose here is to look at a minimum core set of concepts. At this stage only a minimum core set of concepts are considered [137]. 


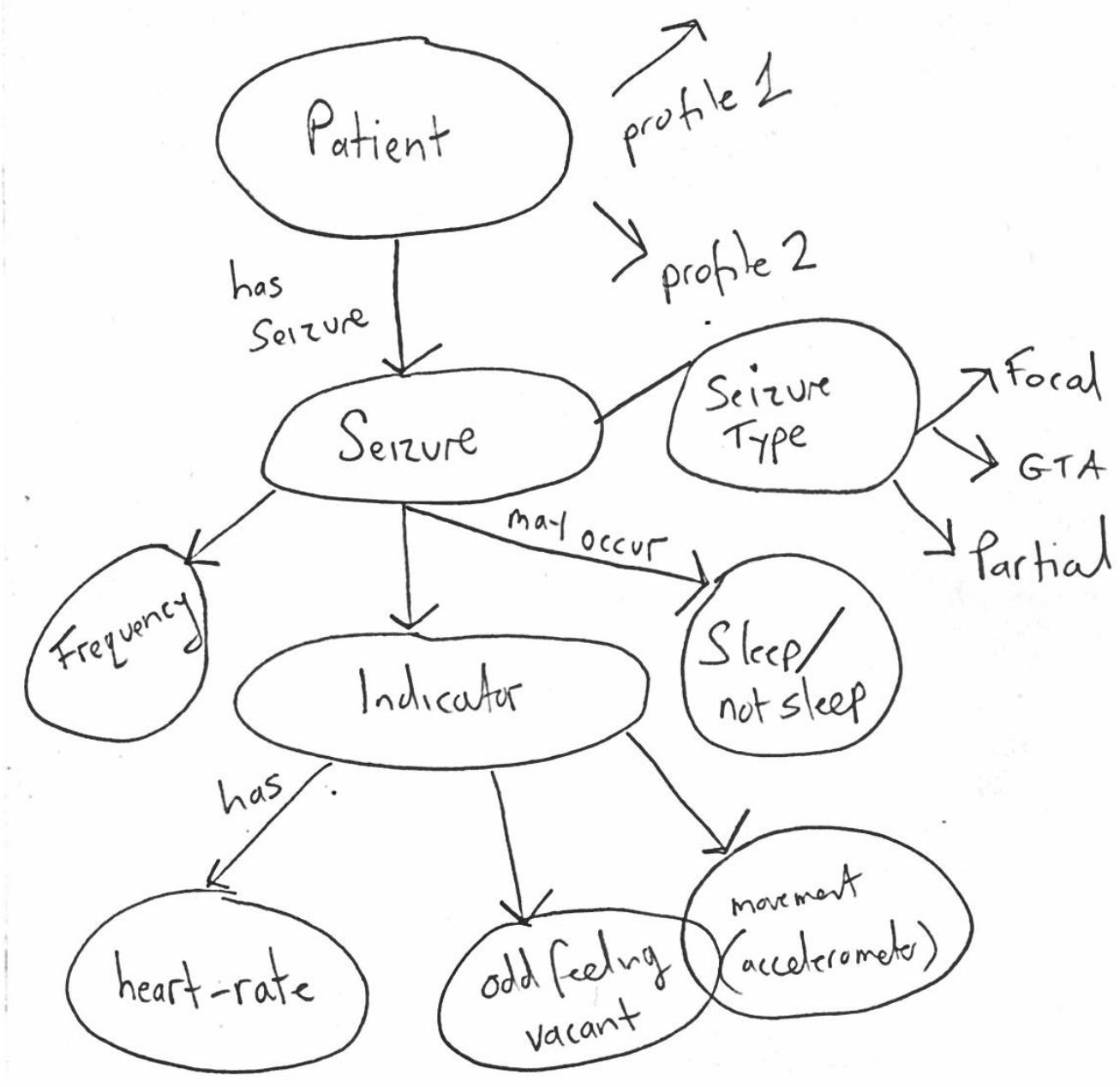

Figure 4.4. Initial Sketch \& Hierarchy

\subsection{Ontology Stage}

The Ontology stage (B1:B3) is discussed below:

\section{B1. Conceptualisation Phase}

The collected vocabulary is at this stage converted into the ontology to add deeper semantics. Any relationships in existing concepts are identified. This is discussed below; first the scope is defined then the classes, properties and attributes are extracted and defined.

B1.1 Defining the Scope

B1.2 Extract Classes \& Attributes/Properties

B1.3 Define the classes and the class hierarchy

B1.4 Define the 'object properties', 'data properties' and 'individuals' 
B1.5. Domains and Ranges

B1.6. Define Data Types and Data Values

B1.7. Make classes disjoint of each other to avoid multiple inheritance

\section{B1.1 Defining the Scope}

The definition of the ontology goal and scope is now considered, this is the first step in the ontology creation. The scope limits the ontology, specifying what must be included and what must not. It is an important step for minimizing the amount of data and concepts to be analysed, especially for the extent and complexity of the ESO. This ontology only considers the needs for creating an 'individual' patient profile with concepts related to the patient's 'seizure' and characteristics specific to their seizure. It does not consider the concepts related to other areas such as the patient's personal details, i.e. weight, height or any other area such as EEG results or other treatments. It only includes concepts related to the actual seizure either before or during with the aim of demonstrating that it is the individual seizure type patient that makes a difference. This is because there is evidence amongst other studies discovered in the literature review (Chapter 2) that whilst recognising the importance of achieving more accurate seizure counts there is also a strong necessity to better distinguish seizure types i.e. between patients exhibiting generalised and partial seizure types [71], also identified is that there is minimal information on which bio-signal or bio-signals combination is best for the individual seizure types and for each individual patient [72].

To demonstrate how the above data is defined Table 4.3. Defining the Scope of the ESO Ontology shows how only the relevant data is selected, the decisions on 'what to include' and what 'not to include' are informed based upon the aforementioned philosophy discussed i.e. concepts relating to the patient's 'seizure'. 
Table 4.3. Defining the Scope of the ESO Ontology

\begin{tabular}{|l|l|l|l|}
\hline $\begin{array}{l}\text { What must be } \\
\text { included? }\end{array}$ & Notes & $\begin{array}{l}\text { What must not be } \\
\text { included? }\end{array}$ & Notes \\
\hline Patient & $\begin{array}{l}\text { The patient and the patient } \\
\text { profile ID must be represented } \\
\text { as the 'individual' }\end{array}$ & $\begin{array}{l}\text { Patient weight, height, } \\
\text { medication etc. }\end{array}$ & $\begin{array}{l}\text { The ontology will not define any } \\
\text { personal characteristics only those } \\
\text { specific to the patient's 'seizure'. }\end{array}$ \\
\hline Seizure_Type & $\begin{array}{l}\text { The type of seizure is } \\
\text { imperative to the individual. } \\
\text { Sometimes the patient has } \\
\text { more than one seizure type. }\end{array}$ & Seizure History & $\begin{array}{l}\text { The history of the patient's seizure } \\
\text { is irrelevant since only its current } \\
\text { occurrence is needed. }\end{array}$ \\
\hline Seizure_Sign & $\begin{array}{l}\text { The seizure sign depicts the } \\
\text { 'start' of the seizure and is } \\
\text { significant as an alert to the } \\
\text { sensor. }\end{array}$ & Seizure Location & $\begin{array}{l}\text { The location of the seizure is useful } \\
\text { to alert carers but the ESO does not } \\
\text { need this to individualise. }\end{array}$ \\
\hline
\end{tabular}




\begin{tabular}{|c|c|c|c|}
\hline Seizure_Symptoms & $\begin{array}{l}\text { The seizure symptoms are } \\
\text { what occurs during the seizure } \\
\text { and will often also alert the } \\
\text { sensor when there is no sign or } \\
\text { alert: i.e. 'right arm shaking'. }\end{array}$ & Seizure Reason & $\begin{array}{l}\text { Sometimes patients begin having } \\
\text { seizures due to illness or accidents, } \\
\text { again this is not useful to the ESO } \\
\text { as it does not depict the individual } \\
\text { seizure type. }\end{array}$ \\
\hline Seizure_Occurances & $\begin{array}{l}\text { How often the seizures occur is } \\
\text { significant to the individual. } \\
\text { This characteristic is specific } \\
\text { to the individual. }\end{array}$ & Seizure EEG results. & $\begin{array}{l}\text { Many seizure EEG datasets exist } \\
\text { yet the emphasis of this study is to } \\
\text { look at remote monitoring as an } \\
\text { 'alternative' to the gold standard } \\
\text { EEG. }\end{array}$ \\
\hline Dominant_Side & $\begin{array}{l}\text { The dominant side, either left } \\
\text { or right will determine which } \\
\text { side the sensor will be worn. }\end{array}$ & $\begin{array}{l}\text { Sleep related disorder } \\
\text { data }\end{array}$ & $\begin{array}{l}\text { Sometime sleep related disorders } \\
\text { can produce symptoms of epileptic } \\
\text { seizures so it was important to } \\
\text { discount this data from patient } \\
\text { records. }\end{array}$ \\
\hline Diurnal_Nocturnal & $\begin{array}{l}\text { Diurnal or nocturnal is whether } \\
\text { the seizures occur at day or } \\
\text { night. This is another } \\
\text { characteristic specific to the } \\
\text { individual. Sometimes this can } \\
\text { be both. }\end{array}$ & Seizure 'duration' & $\begin{array}{l}\text { The duration of the seizure was } \\
\text { considered, yet its length is } \\
\text { insignificant to the creation of the } \\
\text { ESO as it does not specifically } \\
\text { individualise the patient since the } \\
\text { duration is so 'random.' }\end{array}$ \\
\hline
\end{tabular}


In defining this common vocabulary for this specific domain or 'sector' of epilepsy information can be shared. This initial set of data was defined as above, as discussed in the methodology chapter; the clear specifications of domain knowledge are beneficial for new users who must learn the meaning of the terms in the domain [75].

During part of an established step-by-step methodology (Domain Knowledge Acquisition Process: DKAP) [138] when developing an ontology, it is identified that the hierarchy should be defined to represent a structured set of terms; hence in this case to describe the patient seizure type domain which is used as the foundation for a knowledge base [138]. The knowledge in this domain will still evolve among domain experts, therefore 'Epilepsy Specialists' (doctors and nurses) are consulted and several discussions took place together with constant reference to the ILAE guidelines.

The classes, attributes and relations are next developed and guided by the knowledge from the anonymous Patient Profiles (1-100) collected, the ILAE and by adhering to any existing relevant classes and attributes found in other ontologies. This process begins by extracting classes \& attributes to inform the ontology. Following which the relations between these concepts are established, see 'Object Properties' in Table 4.4. Elements of the ESO Ontology. Further details of how this conceptualisation phase is carried out is described below.

\section{B1.2 Extract Classes \& Attributes/Properties}

Prior to extracting the classes and attributes for the ESO important terms in the ontology are enumerated, similar to writing pseudocode before writing a computer program, useful lists of terms are recorded of which can be used either to make statements about or to convey information to the user of the ontology [75] i.e. What are the terms we would like to talk about? What properties do those terms have? What would we like to say about those terms? Much of this initial thinking is mined from the Preliminary Stage. See, Figure 4.5. Initial list of Key Terms in the Hierarchy based upon this aforementioned thinking. 


\begin{tabular}{|c|c|c|}
\hline Classes & Possible Sub-Classes & Relationships \\
\hline Patient & Patient Profile & $\begin{array}{l}\text { Patient 'has a' Patient_Profile } \\
\text { Patient_Profile 'has a' Profile_ID }\end{array}$ \\
\hline $\begin{array}{l}\text { Seizure Sign or } \\
\text { Indicator }\end{array}$ & Nausea & $\begin{array}{l}\text { Patient 'Exhibits' a 'Seizure } \\
\text { Sign' }\end{array}$ \\
\hline $\begin{array}{l}\text { Seizure } \\
\text { Symptoms }\end{array}$ & Awareness & $\begin{array}{l}\text { Patient 'Reveals' 'Seizure } \\
\text { Symptom' }\end{array}$ \\
\hline Seizure Type & FAS & Patient 'Will have' Seizure Type' \\
\hline $\begin{array}{l}\text { Seizure } \\
\text { Occurrences }\end{array}$ & Once a Day & \\
\hline Dominant Side & $\begin{array}{l}\text { Dominant_Left } \\
\text { Dominant_Right }\end{array}$ & Patient 'displays' Dominant Side \\
\hline Diurnal Nocturnal & no subclass, use as instance & $\begin{array}{l}\text { Patient 'seizure time' } \\
\text { Diurnal/Nocturnal }\end{array}$ \\
\hline
\end{tabular}

Figure 4.5. Initial list of Key Terms in the Hierarchy

\section{B1.3 Define the classes and the class hierarchy}

As justified in the Methodology Chapter, a 'top-down' approach is used to develop the class hierarchy [104]. The process starts with creating classes for the general concepts of the patient and seizure. Then it works down the hierarchy and focuses upon the 'seizure class' and considers any subclasses, if the subclass adds no new information it can be removed, and consequently the slot (class) already indirectly contains it [75]. Therefore the 'Patient-Profile' seen as a subclass of Patient in Figure 4.5. Initial list of Key Terms in the Hierarchy' can be removed since Patient represents 'Patient-Profile'. Yet a different thinking can apply with the subclass 'S_Nausea' of 'Seizure_Sign' since:

"If a list of classes defining a range or a domain of a slot contains all subclasses of a class $A$, but not the class $A$ itself, the range should contain only the class $A$ and not the subclasses" [75]

Moreover, 'S_Nausea', should be treated as an 'Individual' (also known as an instance) not a sub-class, as:

"Deciding where classes end, and individual instances begin starts with deciding what is the lowest level of granularity in the representation [75].

Subsequently it is evident that there are no sub-classes in the ontology since it is not concerned with the extension or specific details of i.e. S_Nausea or other signs and symptoms and these elements are the lowest level of granularity therefore they are 'individuals.' These individuals can be seen embedded in Table 4.4. Elements of the ESO Ontology. From Figure 
4.5 created in the previous step terms are selected that describe objects having independent existence rather than terms that describe these objects.

\title{
B1.4 Define the 'object properties', 'data properties' and 'individuals'
}

Next the internal structure of concepts is defined. The classes have already been selected. Then the 'object properties', 'data properties' and 'individuals' are chosen as in Table 4.4. Elements of the ESO Ontology. The full list of individuals for 'Seizure Type and 'Occurrences are shown in Table 4.5. Full List of Individual. Again, this theory is explained in the Methodology Chapter 3. The chosen object properties (also known as relationships or axioms) are defined here (and embedded in Table 4.4):

\author{
$\underline{\text { Relationships (axioms) }}$ \\ Patient 'has a' Patient_Profile \\ Patient_Profile 'has a' Profile_ID \\ Patient 'exhibits' a 'Seizure Sign' \\ Patient 'will have' Seizure Type' \\ Patient 'reveals' 'Seizure Symptom' \\ Patient 'seizure time' Diurnal/Nocturnal
}

\section{B1.5 Domains and Ranges}

Properties may have a domain and a range specified. Properties link individuals from the domain to individuals from the range [111]. Simplistically, the domain and range properties are there to provide an insight into the way that the property links a domain to an object.

To demonstrate this in the ESO ontology, the property 'Has_Sign' links the individual 'Seizure_Sign' belonging to the class Patient. In this case the domain of the 'Has_Sign' property is Patient and the range is 'Siezure_Sign'. This is depicted in Figure 4.6. Domains and Ranges for ESO which shows the Domains and Ranges for the entire ontology together with the Classes, Object Properties and Individuals.

Domains and ranges should not be understood as actual constraints, but as axioms to be used by reasoners [139]. In general, domain for a property is the range for its inverse and the range for a property is the domain of its inverse. As observed to help ensure the coherency of an ontology the use of domains and ranges are vital [140] confirming coherency testing for the basic structure and relationships in the ontology. 


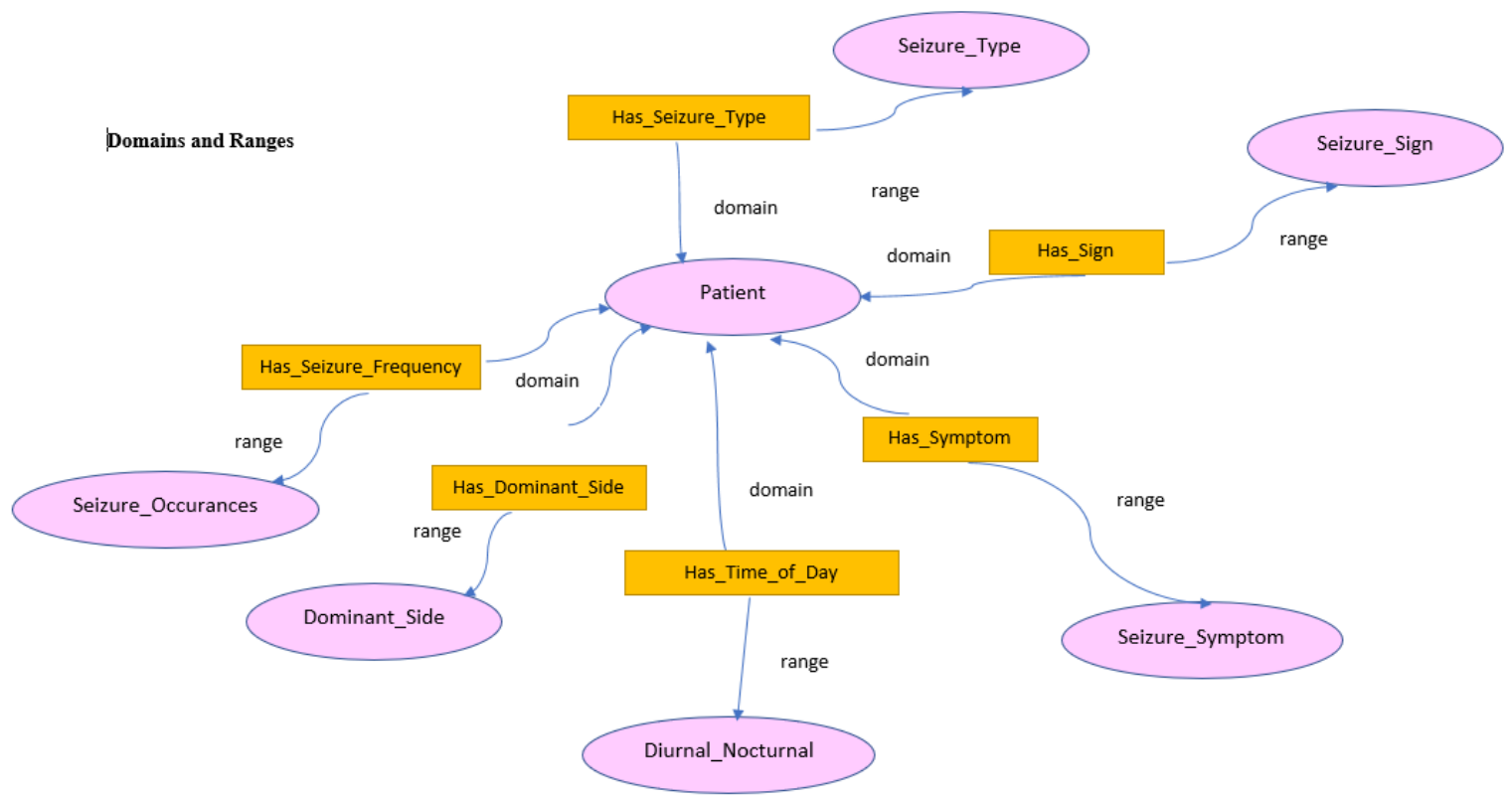

Figure 4.6. Domains and Ranges for ESO

\section{B1.6 Define Data Types and Data Values}

The next stage specifies the data types and data values of the ESO. The datatypes and data values are created and can be depicted in Table 4.4. Elements of the ESO Ontology. 


\begin{tabular}{|c|c|c|c|c|c|c|c|}
\hline Classes & $\begin{array}{l}\text { Sub- } \\
\text { Class }\end{array}$ & $\begin{array}{l}\text { Object Properties } \\
\text { (Relationships) }\end{array}$ & Data Properties & Datatype & $\begin{array}{l}\text { Individuals } \\
\text { (examples) }\end{array}$ & $\begin{array}{l}\text { More than one } \\
\text { instance? } \\
\text { (Individual?) }\end{array}$ & $\begin{array}{l}\text { Data Value } \\
\text { (example) }\end{array}$ \\
\hline Patient & N/A & & Profile_ID & Integer & $1,2,3$ & No & 1 \\
\hline Seizure_Sign & N/A & $\begin{array}{l}\text { Patient 'Has_Sign' a 'Seizure } \\
\text { Sign' }\end{array}$ & Seizure_Sign & String & $\begin{array}{l}\text { S_Nausea } \\
\text { (Etc.) }\end{array}$ & Yes & True/False \\
\hline Seizure_Symptom & N/A & $\begin{array}{l}\text { Patient 'Has_Symptom' } \\
\text { 'Seizure Symptom' }\end{array}$ & Seizure_Symptom & String & $\begin{array}{l}\text { Sy_Awareness } \\
\text { (Etc.) }\end{array}$ & Yes & True/False \\
\hline Seizure_Type & N/A & $\begin{array}{l}\text { Patient 'Has_Seizure_Type' } \\
\text { Seizure Type' }\end{array}$ & Seizure_Type & String & $\begin{array}{l}1 \text { = FMS } \\
\text { (full list in } \\
\text { Table.4.5) }\end{array}$ & Yes & True/False \\
\hline Seizure_Occurances & N/A & $\begin{array}{l}\text { Patient } \\
\text { 'Has_Seizure_Frequency' of } \\
\text { Seizure Occurrences }\end{array}$ & Frequency & String & $\begin{array}{l}\text { Once } \\
\text { daily/nightly }\end{array}$ & No & True \\
\hline
\end{tabular}




\begin{tabular}{|l|l|l|l|l|l|l|l|}
\hline & & & & $\begin{array}{l}\text { (full list in } \\
\text { Table.4.5) }\end{array}$ & \\
\hline Dominant_Side & N/A & $\begin{array}{l}\text { Patient } \\
\text { 'Has_Dominant_Side' } \\
\text { Dominant Side }\end{array}$ & Left_Or_Right & String & Dominant_Left & No & Yominant_Right \\
\hline Diurnal_Nocturnal & N/A & $\begin{array}{l}\text { Patient 'Has_Time_of_Day' } \\
\text { Diurnal/Nocturnal }\end{array}$ & $\begin{array}{l}\text { Diurnal_Or_Noct } \\
\text { urnal }\end{array}$ & String & Diurnal & No & Yes/No \\
\hline
\end{tabular}


Table 4.5. Full List of Individuals

\begin{tabular}{|c|c|}
\hline Seizure Type Individuals & Occurrences Individuals \\
\hline $1=$ FMS & $1=$ once daily/nightly \\
\hline $2=$ FBTCS & $2=$ multiple daily $($ up to 5$)$ \\
\hline $3=\mathrm{GTCS}$ & $3=$ multiple daily $($ over 5$)$ \\
\hline $4=\mathrm{MTC}$ & $4=$ once weekly \\
\hline $5=$ FSIA & $5=$ multiple weekly (up to 5) \\
\hline $6=\mathrm{NMA}$ & $6=$ multiple weekly (over 5) \\
\hline $7=$ unclassified & $7=$ once monthly \\
\hline $8=$ Gelastic & $8=$ multiple monthly (up to 5$)$ \\
\hline $9=\mathrm{GA}$ & $9=$ multiple monthly (over 5) \\
\hline $10=$ FAS & $10=$ once yearly \\
\hline $11=$ Multiple: GTCS/NMA & $11=$ multiple yearly (up to 5) \\
\hline $12=$ Multiple: GTCS/FBTCS & $12=$ multiple yearly $($ over 5$)$ \\
\hline $\begin{array}{l}13=\text { Multiple: GTCS/FSIA } \\
14=\text { Multiple: GTCS/FBTCS }\end{array}$ & $\begin{array}{l}13=\text { just } 1 / 2 \text { occurrences (or in- } \\
\text { frequent) }\end{array}$ \\
\hline $15=$ Multiple: GTCS/FAS & \\
\hline $16=$ Multiple: MTC/GA & \\
\hline $17=$ Multiple: Gelastic/GTCS/FSIA & \\
\hline $18=$ Multiple: GTCS/FBTCS & \\
\hline $19=$ Multiple: GTCS/NMA/FSIA & \\
\hline $20=$ Multiple: GTCS/NMA/GA & \\
\hline $21=$ Multiple: GTCS/NMA/FAS & \\
\hline $22=$ Multiple: GTCS/NMA/GELASTIC & \\
\hline 23 = Multiple: GTCS/EE & \\
\hline
\end{tabular}

\section{B1.7 Make classes disjoint of each other to avoid multiple inheritance}

In preparation for the 'Implementation Phase' the next step is to make classes disjoint of each other to avoid multiple inheritance, so that then there will only be an instance of 'one' class. As discussed in the Methodology Chapter the benefits of avoiding multiple inheritance 
deliver a reduced loss of information by using a more precise representation and provide an ontology that has less errors, that can be used for automated reasoning and is easier to maintain [115]. Consequently, the collected ontology concepts are inspected, and classes are made 'disjoint' of one another, see Table 4.6.

Table 4.6. Disjoints

\begin{tabular}{|c|c|}
\hline Class & Disjoints With \\
\hline Patient & $\begin{array}{l}\sqrt{ } \text { Seizure_Sign, Seizure_Symptoms, } \\
\text { Seizure_Type, Seizure_Occurances, } \\
\text { Dominant_Side, Diurnal_Nocturnal }\end{array}$ \\
\hline Seizure_Sign & $\begin{array}{l}\sqrt{ } \text { Patient, Seizure_Symptoms, } \\
\text { Seizure_Type, Seizure_Occurances, } \\
\text { Dominant_Side, Diurnal_Nocturnal }\end{array}$ \\
\hline Seizure_Symptom & $\begin{array}{l}\sqrt{ } \text { Patient, Seizure_Sign, Seizure_Type, } \\
\text { Seizure_Occurances, Dominant_Side, } \\
\text { Diurnal_Nocturnal }\end{array}$ \\
\hline Seizure_Type & $\begin{array}{l}\sqrt{ } \text { Patient, Seizure_Sign, } \\
\text { Seizure_Symptoms, } \\
\text { Seizure_Occurances, Dominant_Side, } \\
\text { Diurnal_Nocturnal }\end{array}$ \\
\hline Seizure_Occurances & $\begin{array}{l}\sqrt{ } \text { Patient, Seizure_Sign, } \\
\text { Seizure_Symptoms, Seizure_Type, } \\
\text { Dominant_Side, Diurnal_Nocturnal }\end{array}$ \\
\hline Dominant_Side & $\begin{array}{l}\sqrt{ } \text { Patient, Seizure_Sign, } \\
\text { Seizure_Symptoms, Seizure_Type, } \\
\text { Seizure_Occurances, Diurnal_Nocturnal }\end{array}$ \\
\hline Diurnal_Nocturnal & $\begin{array}{l}\sqrt{ } \text { Patient, Seizure_Sign, } \\
\text { Seizure_Symptoms, Seizure_Type, } \\
\text { Seizure_Occurances, Dominant_Side }\end{array}$ \\
\hline
\end{tabular}




\section{B2. Implementation Phase}

Protégé 5 is used to implement the ontology. It includes the concepts and properties that characterize the data extracted from the analysis of the 100 anonymous patient records carried out in the previous stages. Protégé is an ontology development tool (discussed in Methodology Chapter) and is used to build and edit the ontology.

Now that the design of the ontology has been decided, the next stage in the process is to build the ontology with Protégé 5. This sequential process can be seen in Figure 4.7. Protégé Implementation Steps. Using Table 4.4. Elements of the ESO Ontology to guide the development the steps in Figure 4.7 are carried out. The steps are divided and discussed in the following sections:

B2.1 Specify IRI

B2.2 Implement the Ontology

B2.3 Visualisation Tools

B2.4 Exporting to Web Ontology Language (OWL) \& XML

B2.5 Publish The ESO

\section{Protégé Implementation Steps}

1. Specify Ontology IRI (put on webspace) to store ontology there

2. Setup classes and sub classes

3. Make classes disjoint of each other to avoid multiple inheritance (so will only be an instance of one class)

4. Create Object properties (describing relationships between 2 properties of individuals)

i.e. "has a ": a patient "has" a seizure type

a patient "has a" seizure type

5. Specify the domain and range for object property

6. Next is "Data Properties" which describes relationships between instance and data values for example seizure type has a certain name or code. Or Patient has "profile ID"

7. Run Reasoner, check everything is working and makes sense.

8. Next is 'Adding Individuals': individuals represent the instances from the classes.

Figure 4.7. Protégé Implementation Steps 


\section{B2.1 Specify IRI}

To begin the ontology set-up first the IRI is specified. Each named ontology has a specific IRI (Internationalized Resource Identifier) and the Ontology IRI should be an absolute IRI. For the present ontology, as recommended the Ontology IRI is in fact a URL (Uniform Resource Locator) (http://..........) This points to the web location from where the ontology can be downloaded.

Protégé allows the Ontology IRI to be viewed and edited. The Ontology IRI also has an Ontology Version IRI. The version IRI describes the version of the ontology and this version IRI is also a URL that points to the Web location where this version of the ontology will be published.

\section{B2.2 Implement the Ontology}

Guided by Table 4.4. Elements of the ESO Ontology, the next steps in the ontology development are carried out, from step 2 to 8 (Figure 4.7).

The resulting ontology can be seen in this overall view in Figure 4.8. Ontology Implementation Snapshot. 
Classes

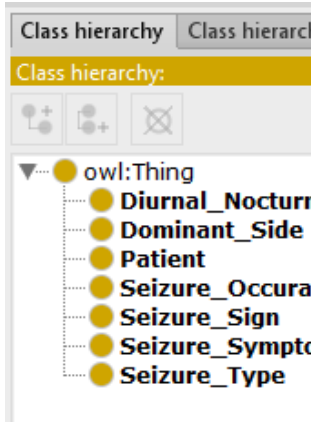

Object

Properties

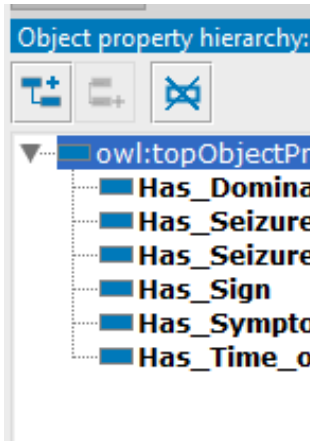

Disjoints

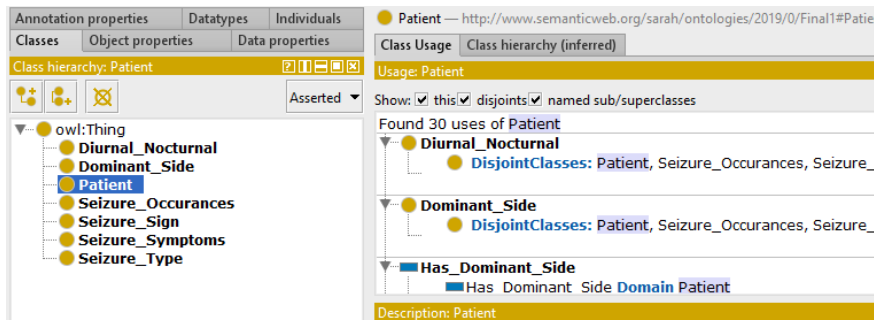

Data

Individuals

Properties

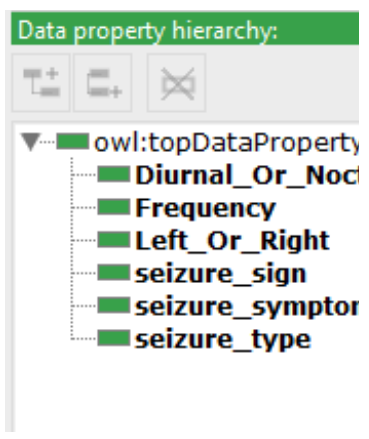

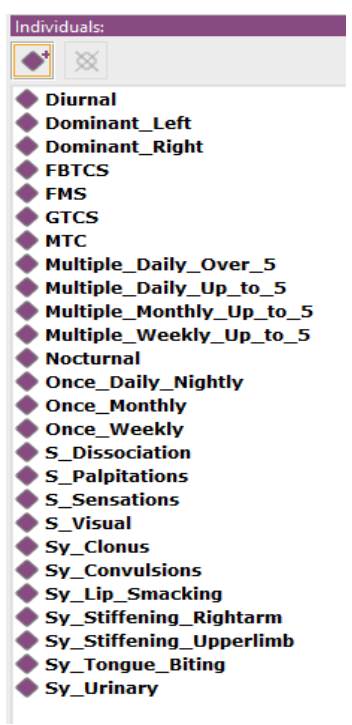

Figure 4.8. Ontology Implementation Snapshot

\section{B2.3 Visualisation Tools}

The structure of the ESO, can be visualised below, using Ontograph (see Figure 4.9. Ontograph Visualisation below).

The relationships are interactively navigated within the ontology. The key below the ontology (Arc Types) shows the different relationships; subclass, individual, domain/range object properties, and equivalence. In this case there are no equivalence found in that no two classes had the same individuals in any interpretation, due to naming convention i.e. S_Visual $=$ Sign_Visual and Sy_Visual=Symptom_Visual. The Ontograph aids in this analysis: for object and data properties, asserting that two properties are equivalent means that their domains and ranges apply to both properties, and that every assertion using one property can be rewritten as using the other [141]. With reference to Figure 4.9: the 'classes' are 
represented with the orange symbols together with their 'individuals' (purple diamonds). While the colour key in the pane beneath (Arc Types) shows the relationships between the 'classes' and 'individuals', namely the 'object properties'.

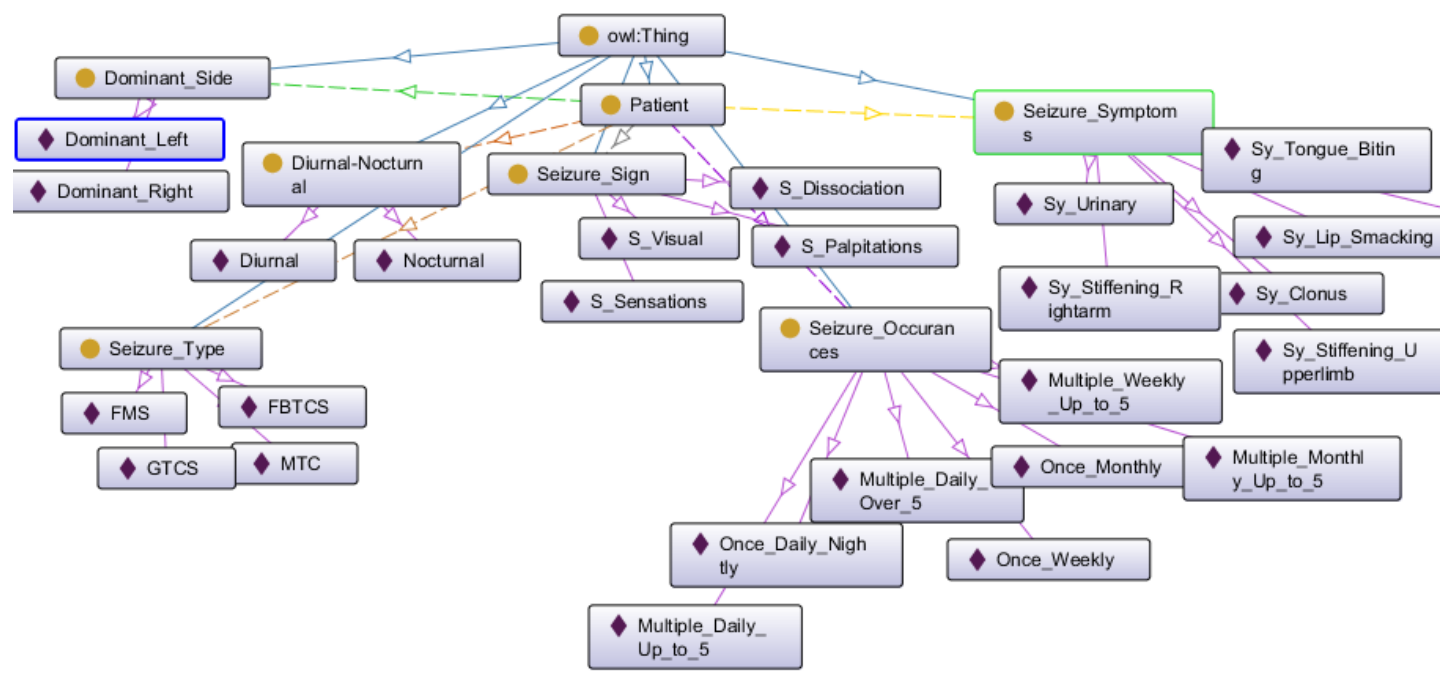

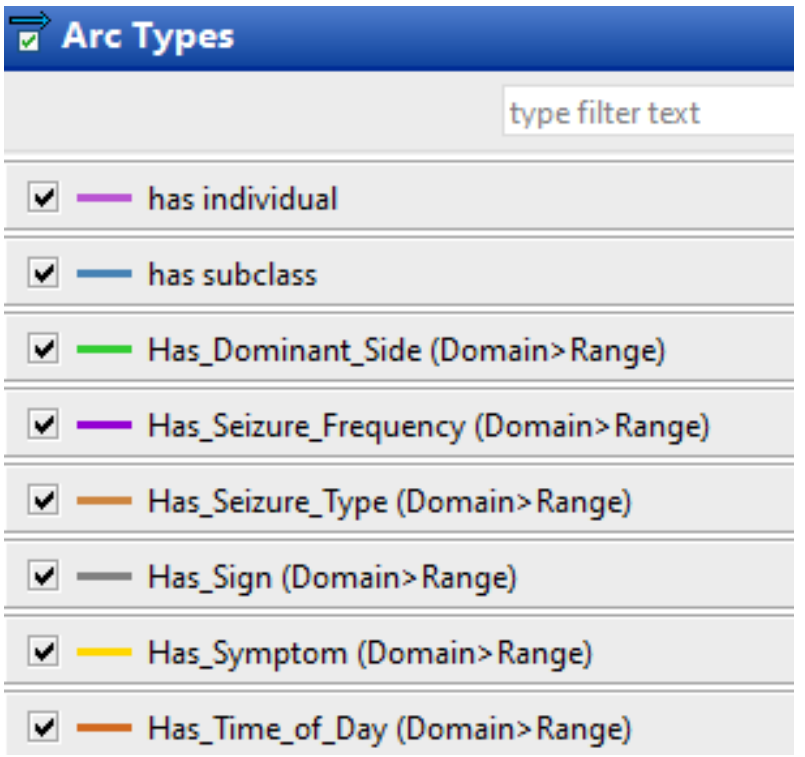

Figure 4.9. Ontograph Visualisation

The Protégé VOWL plugin is also used to provide a 'user-oriented' visualization of the ESO ontology, see Figure 4.10. VOWL Visualisation. It implements the 'Visual Notation for OWL Ontologies' (VOWL).

The Visual Notation for OWL Ontologies (VOWL) defines a visual language for the useroriented representation of ontologies. It provides graphical depictions for elements of the Web Ontology Language (OWL) that are combined to a force-directed graph layout visualizing the ontology [142].

This visualisation clearly focuses on the ontology schema (i.e. The classes, properties and datatypes). Connected by lines, the circles depict the classes and the arrows represent the property relations. Property labels and datatypes are shown in rectangles. Information on 
individuals and data values is either displayed in the visualization itself or in another part of the user interface.

The second ingredient of VOWL is a colour scheme complementing the graphical primitives. It defines colours for the visual elements to allow for an easy distinction of different types of classes and properties. The Protégé VOWL visualisation is used to present the ESO ontology in a user-friendly way and helps provide an overall 'Birdseye view' image.

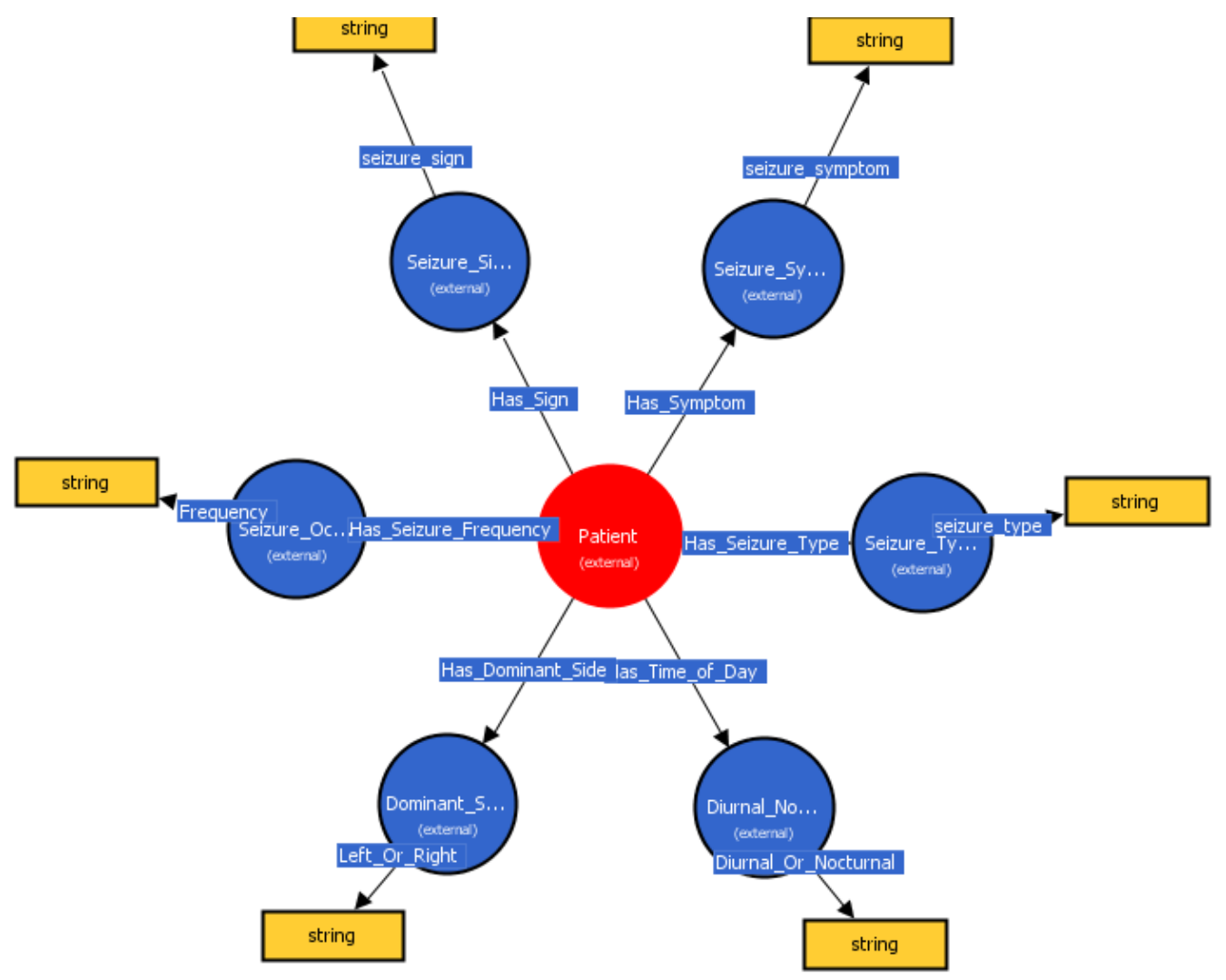

Figure 4.10. VOWL Visualisation

\section{B2.4 Exporting as ESO OWL and XML Serialization for PPDL}

As revealed in the Methodology Chapter 3, embracing the semantic web is an important consideration for the future of the ESO, as further observed recently that there has been an interest in presenting ontologies using ontology languages such as the Web Ontology Language (OWL) [143].

Also expressed recently is the observation that the success of the Semantic Web depends strongly on the growing amount of ontologies. Because of this reliability upon such formal ontologies there is also an impact on the structure underlying data for the purpose of comprehensive and transportable machine understanding [144] hence demonstrating the important role of ontologies on the Semantic Web. 
Today ontologies are part of the W3C standards stack for the Semantic Web, providing users with the necessary structure to link one piece of information to other pieces of information on the Web of Linked Data [122].

Subsequently the ESO ontology is saved as a Web Ontology Language (OWL) and is thus transformed to a 'Semantic Ontology.' The OWL format, which is synonymous with Protégé is in a "pure" RFD (Resource Description Framework) format, which was also discussed in the Methodology Chapter 3.

Before saving the ontology into an OWL file it is a necessary requirement to run the reasoner (see B3 later) first and fix any errors prior to this. There are different syntaxes for OWL in Protégée which first prompt the user to choose the version. The default is RDF/XML and is the best format to choose, since it is the most stable in Protégé [145]. To import the Ontology to a web ontology (OWL file) the process is straight forward: using File>Save or File>Save as.

Even though an ontology is used to describe knowledge in a certain domain, nonetheless the key is to keep in mind the three major uses of ontologies identified, to assist in communication between humans, to achieve interoperability, and to facilitate communication among software systems [146].

In order to achieve this for ESO and make it useable for HCPs (Health Care Professionals) it needs to be in a language that is understandable by humans and machines, [147] this can be accomplished by XML.

Moreover, it is important to 'retain' the metadata schema from the ontology, this can be done with RDF serialisation. In addition, the vocabulary of the concepts used in the annotation can be transformed into semantic annotations.

There are several ways Protégé achieves this serialisation. Firstly, the OWL ontology is mapped to a set of RDF triples [147] then the RDF can be serialised in different formats, so in essence the outcome is the RDF/XML serialisation of the RFD mapping of an OWL ontology (OWL file). These serialisations include, Turtle, N3, RDFa (RDF embedded in HTML) and RDF/XML.

Hence it is common to find that the OWL files which exist online are in fact RDF/XML serializations of RDF mappings of OWL ontologies [148].

Therefore, ESO is transformed (during the saving process mentioned earlier) into RDF/XML serialisation. This XML becomes an outcome: namely the PPDL (Patient Profile Description Language).

The ESO (Epilepsy Seizure Ontology) is used to inform the PPDL Language in XML. 


\section{B2.5 Publishing the ESO}

The latest version of the ESO ontology is published with its Ontology IRI. PPDL is freely available at http://www.epilepsypatient.co.uk/PPDL.owl a snapshot is shown in Figure 4.11. Snapshot of Raw XML PPDL. This ontology, once evaluated, will be been submitted to the Bioportal site of NCBO.

Protégé uses a hash (“\#”) by default to identify the classes and properties that are declared in the vocabulary/ontology. A slash ("/") for this purpose is used as well.

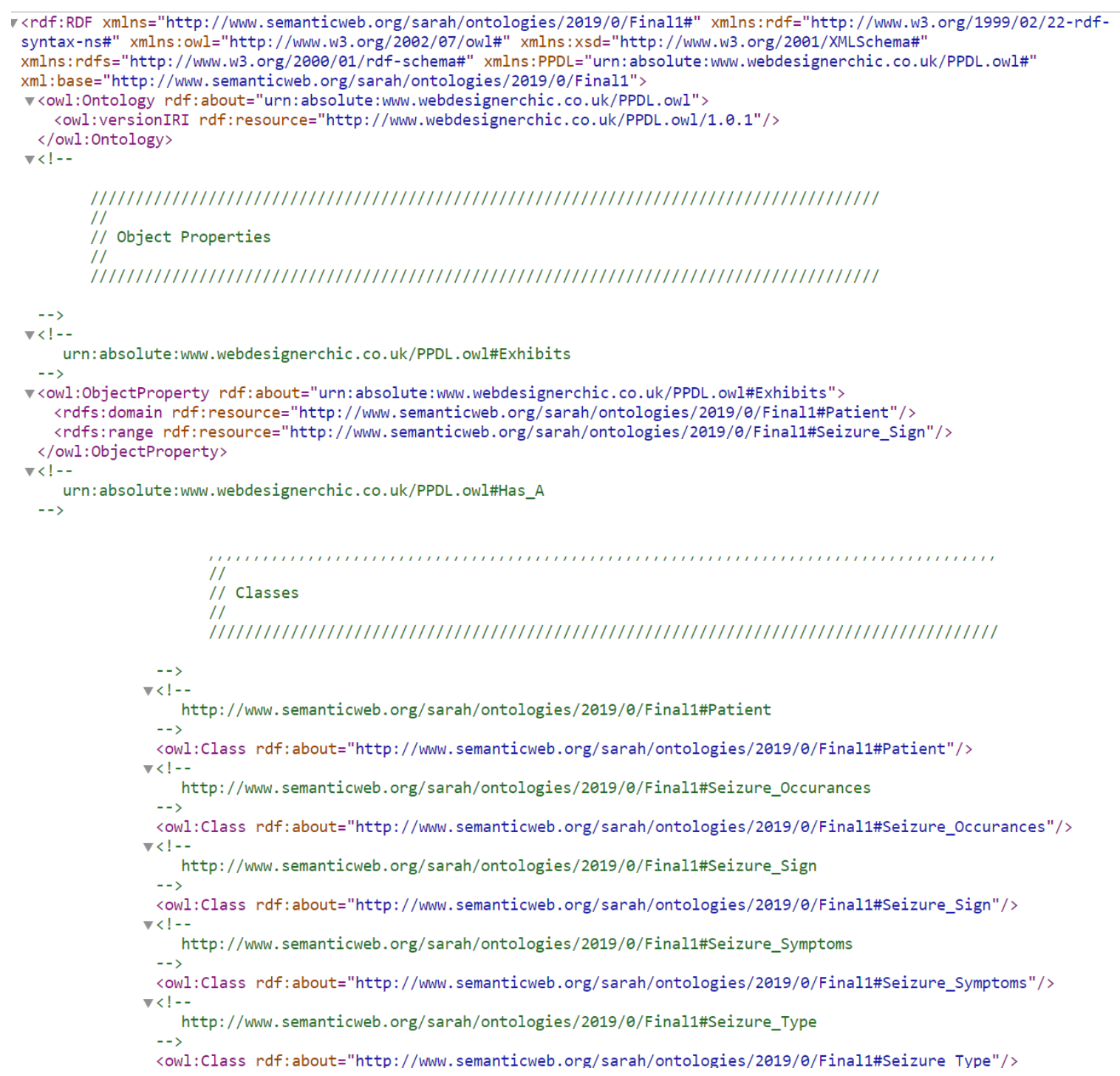

Figure 4.11. Snapshot of Raw XML PPDL

\section{B3. Ontology Evaluation Phase}

In this section tools used to debug the ontology and remove errors are demonstrated. Finally, to evaluate the ontology a review by domain experts for accuracy and completeness of the knowledge represented in the ontologies is undertaken.

The steps are divided and discussed in the following sections: 
B3.1 Reasoners in Protégé

B3.2 Protégé Debugger

B3.3 Evaluation Tools

B3.4 Review by Experts

\section{B3.1 Reasoners in Protégé}

The importance of quality and correctness of ontologies in semantic representation and knowledge sharing was discusses in the Methodology Chapter 3.

To determine the effect of axioms in more expressive logics, i.e., OWL 2 DL, the fact++ reasoner was used to attempt to classify the ontology. This fact++ reasoner can reason about Web Ontology Language (OWL) and Resource Description Framework (RDF) constructors, deducing new facts in ontologies.

The results of the reasoner did not reveal any additional unsatisfactory classes or new facts, see Figure 4.12. Reasoner Log below.

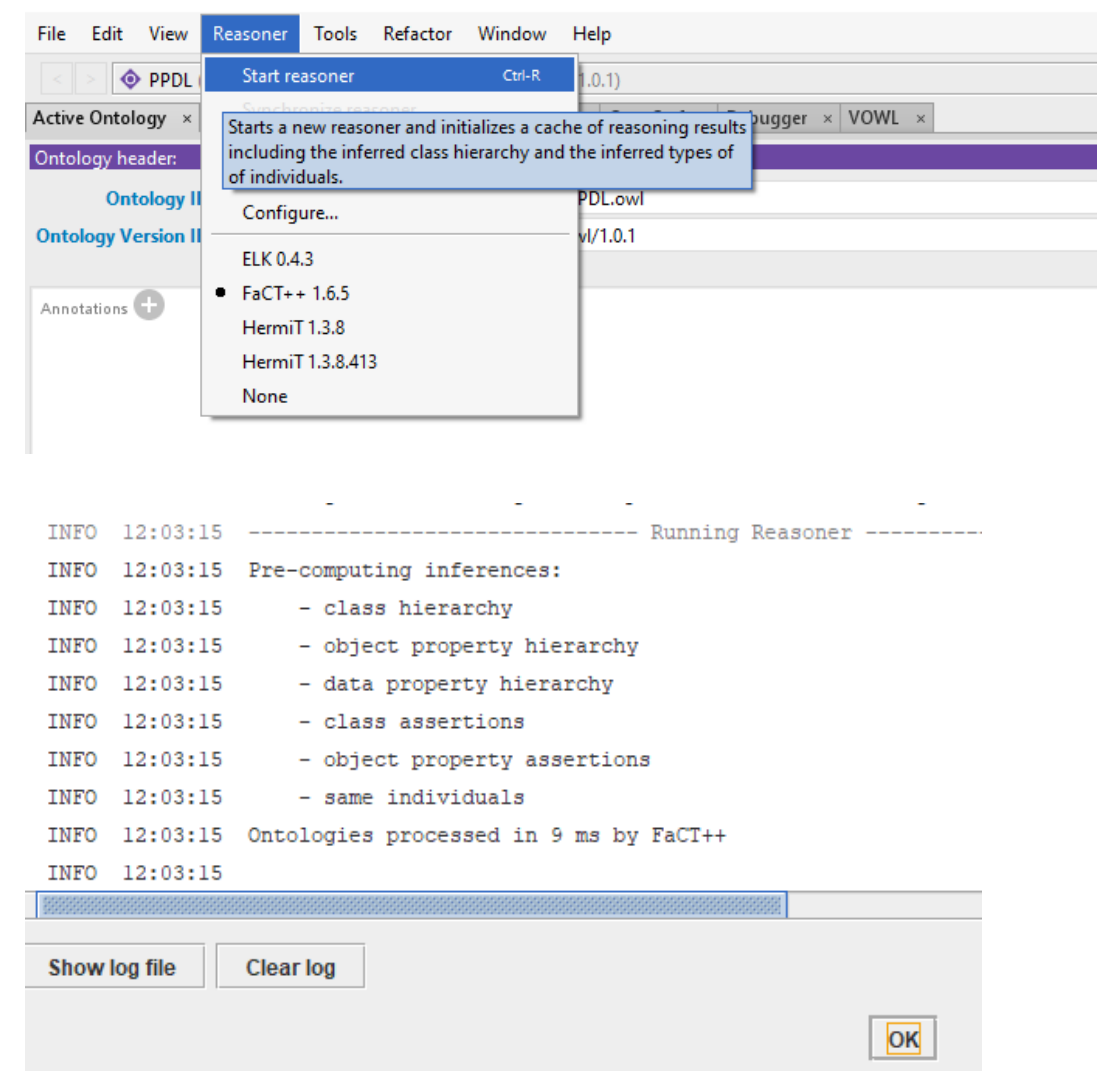

Figure 4.12. Reasoner Log 


\section{B3.2 Protégé Debugger}

The debugger in Protégé is also used to check if the ontology is coherent and consistent, as evidenced in Figure 4.13. Coherent Ontology.

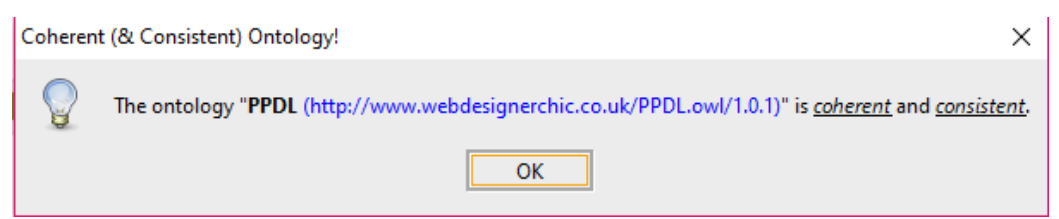

Figure 4.13. Coherent Ontology

\section{B3.3 Evaluation Tools}

As observed in the Methodology Chapter 3 when evaluating ontologies some anomalies can appear. Therefore, in any ontology development project it is vital to perform the ontology evaluation activity since this activity checks the technical quality of an ontology against a frame of reference [129].

Evaluation tools were also previously analysed and the Ontology Pitfall Scanner (OOPS!) was chosen, as observed it depends on the purpose of the evaluation which approach is the most suitable [149]. For example, to check the consistency of the ontology, the reasoner has already been used, in addition the coherence of its representation according to domain knowledge, will be checked working closely with domain experts. For the next step: correctness and completeness OOPS is a useful and easy to use tool for supporting ontology evaluation [150].

Also as observed compared to OOPS most other evaluation methods for an ontology don't show any tangible ontology diagnosis output and tend only to deal with taxonomical knowledge, address a narrow range of ontology evaluation aspects or provide a set of measurements [151]. Henceforth with OOPS there is an output to work with and correct directly.

\section{B.3.3.1 Ontology Pitfall Scanner (OOPS!)}

\section{co:5/ OntOlogy Pitfall Scanner!}

Figure 4.14. Pitfall Scanner

In order to produce a list of evaluation results, the Ontology Pitfall Scanner [152], see Figure 4.14 takes as input the ontology to be analysed. The system is accessed by a web user interface and to machines throughout a web restful service. The input ontology can be entered by its URI or the OWL code 2, which describes the ontology to be analysed. Once the ontology is 
parsed using the Jena API3, the Pitfall Scanner module inspects the declared ontology looking for pitfalls among those available in the catalogue [129].

\section{B.3.3.2 Scanner Results and Evaluation}

At the time of writing this thesis all 41 of the 41 pitfalls defined in the catalogue are evaluated within the system, see Figure 4.15. Pitfalls Defined. During this scanning phase, the ontology elements prone to potential errors are detected.

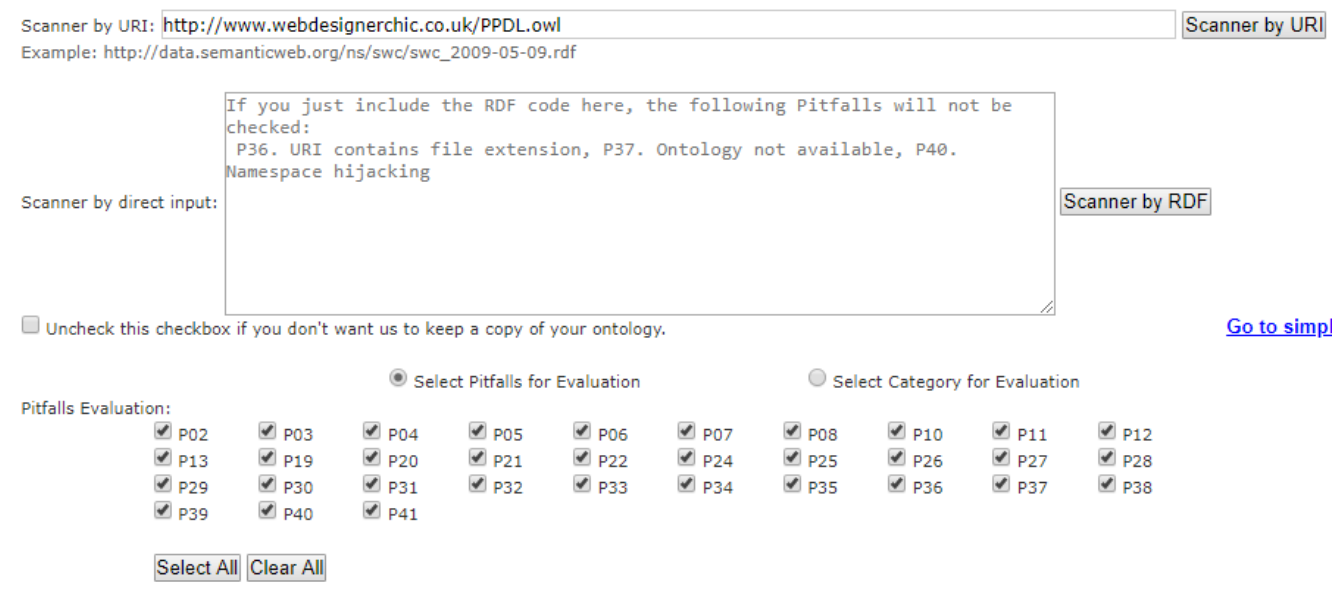

Figure 4.15. Pitfalls Defined

The evaluation results from the Pitfall Scanner are provided from OOPs and include a list of pitfalls detected, and the ontology elements affected, as well as explanations describing the findings. This first set of results was obtained using the original IRI shown in the screenshots, this IRI has since been updated to: http://www.epilepsypatient.co.uk/PPDL.owl Within the first set of results 1 critical case was found, (P19) according to the pitfall scanner it is crucial to correct the pitfall otherwise it could affect the ontology consistency, reasoning and applicability. The critical case found was that more than one domain or range statements was defined by an Object Property. In this Critical Case the object property identified: 'Yes_No' had been shared between several of the classes, 'Seizure_Sign', 'Diurnal_Nocturnal'). This Object property 'Yes_No' was removed, it was later discovered it was essentially redundant and new Object Properties where created to uniquely describe the relationships between classes i.e. 'Has_Sign', 'Has_Time_of_Day' etc. Hence evaluation results below depicted in the Pitfall Scanner, see Figure 4.16. Critical Case.

Results for P19: Defining multiple domains or ranges in properties. 1 case $\mid$

Critical 으

The domain or range (or both) of a property (relationships and attributes) is

defined by stating more than one rdfs:domain or rdfs:range statements. In OWL

multiple rdfs:domain or $r d f$ s:range axioms are allowed, but they are interpreted as

Figure 4.16. Critical Case 
Upon fixing the critical case the ontology was put through the scanner again and the second results revealed the critical case fixed with only 2 important and several minor errors see Figure 4.17. Results 2.

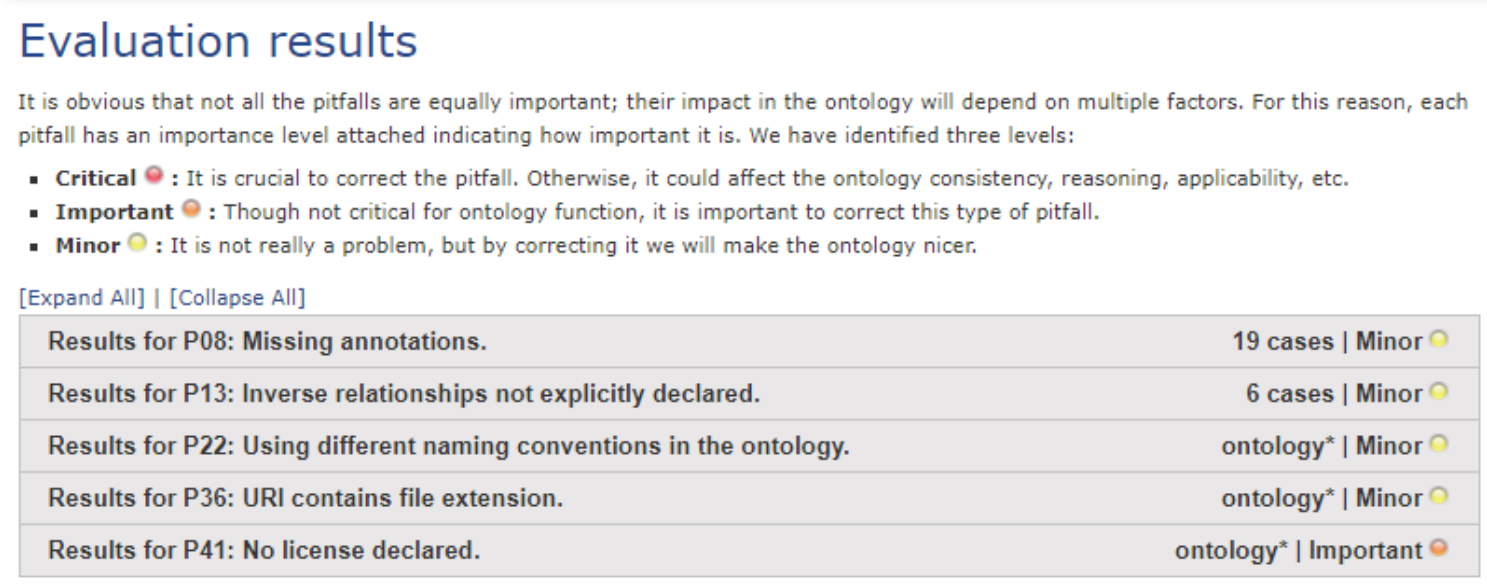

Figure 4.17. Results 2

The 19 cases (Minor) revealed that annotations where missing; this was fixed see Figure 4.18. Annotations Fixed. Giving human-understandable documentation to OWL entities is important and non-trivial [152].

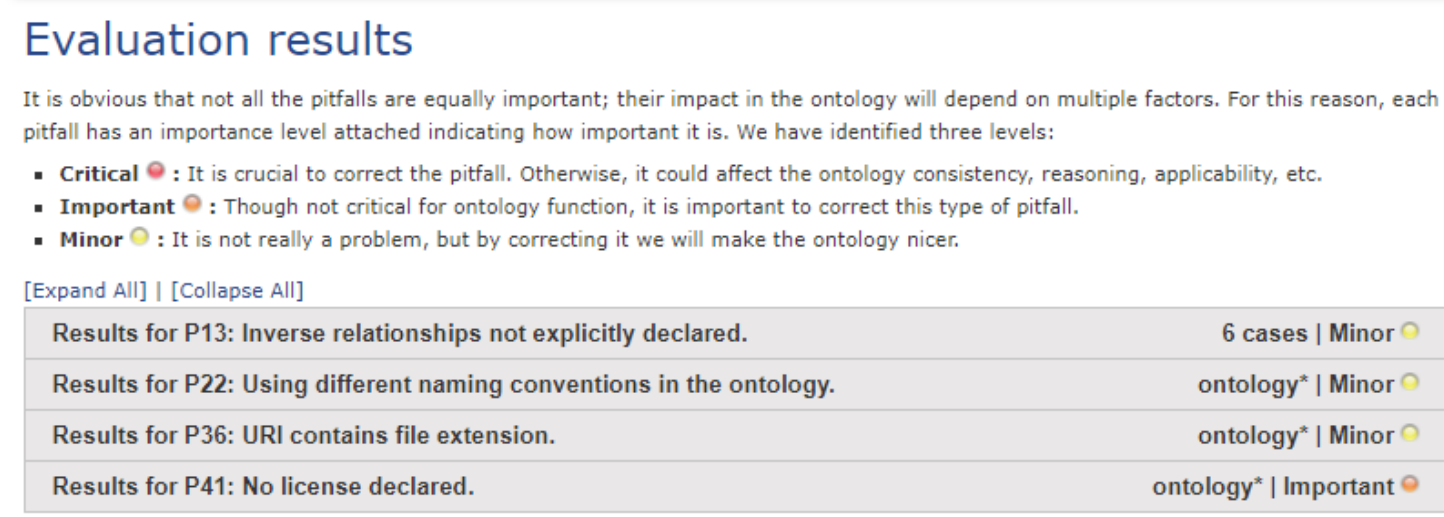

Figure 4.18. Annotations Fixed

The next Minor Cases: P13 : "Inverse relationships not explicitly declared". In the 6 Object properties declared within the Ontology there exists no inverse relationships. All current relationships are independent of each other, as below Figure 4.19. No Inverse Relationships. 


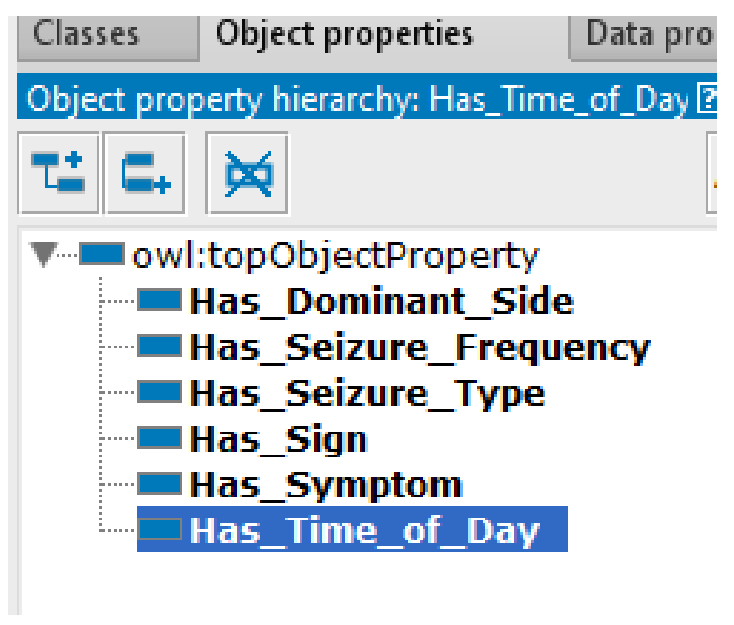

Figure 4.19. No Inverse Relationships

One of the other Minor Cases: 'Using different naming conventions in the Ontology' was related to use of delimitators i.e. As "-" or "_", in Figure 4.20. Use of Deliminators below.

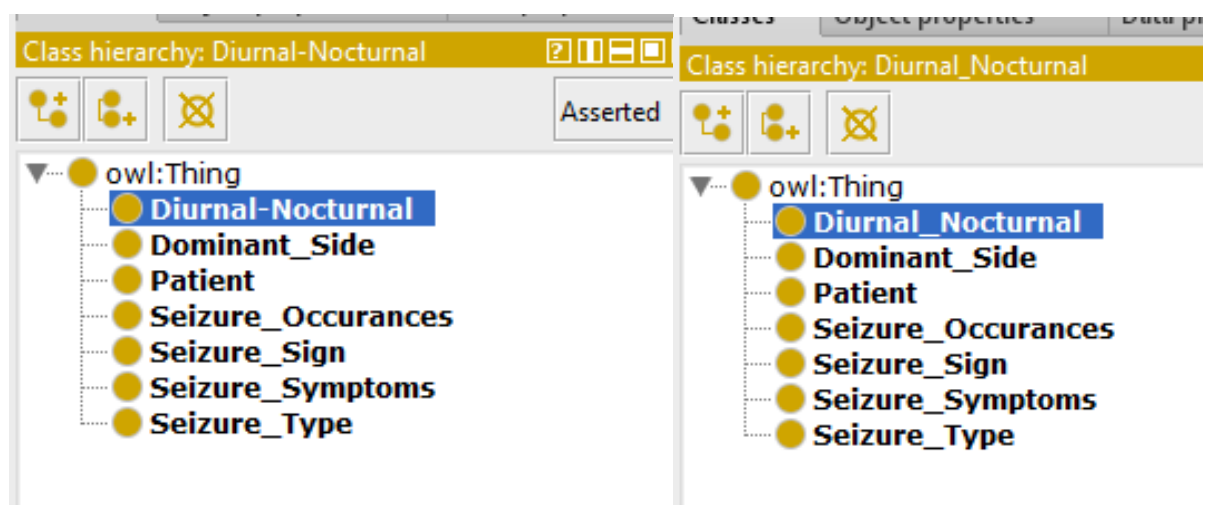

Figure 4.20. Use of Deliminators

Apparent is that the entity 'Diurnal-Nocturnal' needed to be updated, as indicated by fix. The final minor case: 'URI contains files Extension', this pitfall occurs if file extensions such as ".owl", ".rdf", ".ttl", ".n3" and ".rdfxml" are included in an ontology URI. Best practices indicated reveal that a URI should be persistent, Archer et al claim in their:'10 rules for persistent URIS' that it is necessary to remove the extensions as;

- Uris are dumb strings, i.e. They carry no meaning except to identify a resource. For clarity, a URI such as http://example.com/document.pdf does not convey that there is a PDF available at that location. It would be perfectly conformant, contrary to what one would expect, for this URI to return a CSS stylesheet.

- Servers are smart and flexible - they can be configured to do a great deal more than return a static file and such configuration means that a single URI might de-reference to different resources in future. [153]

Archer et al conclude that since few things change as rapidly as technology these facets of HTTP mean that it can be said that persistent URI's should not include file extensions because 
often (although not necessarily) they reveal the technology used to create the resource. Yet the Protégé tool used in this thesis does not allow the facility to remove a file extension, furthermore without the extension the OOPS scanner cannot read the ontology. Since this is deemed as a 'minor case' the extension will remain.

Finally, the 'Important Case': 'No Licence declared', the ontology metadata omits information about the license that applies to the ontology. As an ontology is after all 'content', a 'Creative Commons License' would be appropriate. The Gene Ontology, for example, uses a 'Creative Commons Attribution 4.0 Unported License [154]. It is the intention to acquire a licence for this ontology once the study is finalised. Consequently, to conclude the evaluation using the pitfall scanner, the conformance badge provided reads: "according to the highest importance level of pitfall found in your ontology the conformance badge suggested is "Important pitfalls": see Figure 4.21. Conformance Badge.

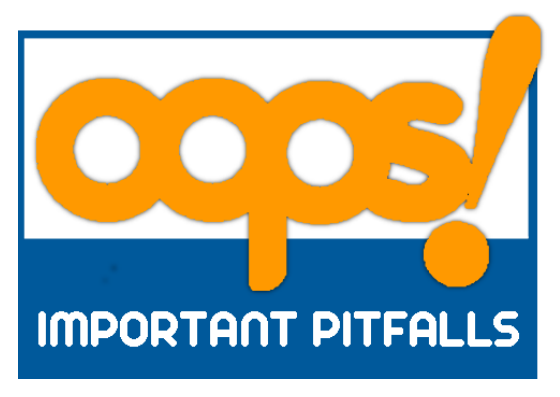

Figure 4.21. Conformance Badge

\section{B3.4 Review by Experts}

Four experts in the field of Epilepsy are consulted to review the final ontology. Working closely with 'The Walton Centre Neurosurgery Department' provided valuable assurance and helped to clarify any ambiguities throughout the development of the ontology. This close collaboration helped to build a data model fit for real-world adoption inside hospital settings.

The ontology reviewed by the above four domain experts, can be seen in Table 4.7. Epilepsy Expert Review together with the ontology questions.

The answers to the 4 sets of evaluation questions were 100\% with yes: positive, indicating that indeed ESO is a sound and useable ontology. Conversations occurred regarding the ESO prior to the clinicians completing the form, these are also recorded in Table 4.7. Epilepsy Expert Review under 'Verbal Feedback'. In general, the initial reactions from clinicians were of pleasant surprise and genuine appreciation in the fact that the terminology was familiar and useful to them. The clinicians also expressed how the ESO could be added to in the future and how some of the updated ILAE abbreviations were important as the epilepsy terminology evolves.

Overall the evaluation forms together with the verbal feedback indicated the ontology model is a true representation of the everyday real-world properties of an individual epilepsy patient. 
Table 4.7. Epilepsy Expert Review

\begin{tabular}{|c|c|}
\hline Evaluation Questions & $\begin{array}{l}\text { Reviewed by Domain } \\
\text { Experts }\end{array}$ \\
\hline $\begin{array}{l}\text { Q1. Can the knowledge } \\
\text { depicted in the ontology } \\
\text { determine if an individual } \\
\text { patient profile is } \\
\text { represented? }\end{array}$ & $\begin{array}{l}\text { Yes. } \\
\text { Verbal feedback } \\
\text { The attributes represent the } \\
\text { terms we are familiar with. } \\
\text { They are recognisable from } \\
\text { the patient medical records: } \\
\text { information we read every } \\
\text { day. }\end{array}$ \\
\hline $\begin{array}{l}\text { Q2. Can the knowledge } \\
\text { depicted in the ontology } \\
\text { determine the feasibility } \\
\text { of the ontology? }\end{array}$ & $\begin{array}{l}\text { Yes } \\
\text { Verbal feedback } \\
\text { The way that the terms have } \\
\text { been categorised is a good } \\
\text { way to view the data. }\end{array}$ \\
\hline $\begin{array}{l}\text { Q3. Can the concepts } \\
\text { presented in the Ontology } \\
\text { easily evolve in the } \\
\text { future? (be added to?) }\end{array}$ & $\begin{array}{l}\text { Yes } \\
\text { Verbal feedback } \\
\text { It is possible to add further } \\
\text { categories if required. }\end{array}$ \\
\hline $\begin{array}{l}\text { Q4. Do the classes } \\
\text { represent a sound and } \\
\text { accurate depiction of a } \\
\text { 'Patient Profile'? }\end{array}$ & $\begin{array}{l}\text { Yes } \\
\text { There is nothing extra that } \\
\text { should be included. }\end{array}$ \\
\hline
\end{tabular}


Q5. Do the updated

abbreviations and

terminology conform to

ILAE and are they clearly

understood?
Yes

Some of the abbreviations are

being used but not all.

\subsection{Summary}

This chapter discussed the implementation of the ESO (Epilepsy Seizure Ontology) and demonstrated its use to inform the PPDL Language in XML. From the initial 'obtaining knowledge' stage through to its evaluation the PPDL has presented a strong component to be incorporated in the PMP framework developed later in the thesis. 


\section{Chapter 5 Clustering}

\subsection{Introduction}

This chapter begins by discussing the reasoning behind clustering the patient profiles to fulfil Objective 3: 'Perform a Clustering technique to discover distinct groups of patients that share similar characteristics.' The rest of this chapter discusses the methods outlined in the Methodology Chapter for the Clustering Stage: (C1-C2) by discussing a selection of clustering methods and algorithms (C1) then it continues to the next step: (C2) whereby Clustering is performed.

\subsection{Clustering Stage (III)}

The Clustering stage consists of 2 activities (C1-C2)

\section{C1. Clustering Analysis}

This stage carries out an analysis on the concept of clustering, together with tools, techniques and algorithms used.

\section{C1.1 Clustering Analysis overview}

Clustering methods have been used in many similar scenarios whereby a diversity of patent characteristics have endeavoured to be understood: i.e. Irritable bowel syndrome [155] diabetes [156] and asthma [157] but so far little is known about any associations for epilepsy patient profiles that share multiple characteristics in sub-groups to achieve a more personalised care.

This section identifies systematic patterns of perceptions using cluster analysis, and investigates associations among epilepsy patient profile clusters, for example patients who have the seizure type ' $G A$ ' will frequently fall into the category of having seizures that occur daily.

The ESO (Epilepsy Seizure Ontology) dataset developed in Chapter 4 uncovered a variety of patient profiles showing similarities. However, clustering techniques provide a replicable methodology for grouping the patient's profiles. Using clustering techniques, grouping becomes feasible and replicable, shifting from a "one size fits all" [158] to a more individual, patient-tailored approach and thus improve health outcomes [158].

\section{C1.2 Clustering Techniques}

Prior to performing Clustering, next is an analysis of relevant clustering techniques. In 1967 P.E Green alleged 'Clustering Analysis' is a set of techniques used to identify "similar" 
entities from characteristics (categorical or measured) possessed by the entities [159]. Today clustering analysis is still very much in use and is 'one of the most common exploratory data analysis technique used to get an intuition about the structure of the data' [160] and can be defined as the task of identifying subgroups in the data such that data points in the same subgroup (cluster) are very similar while data points in different clusters are very different.

It is important for a HCP to diagnose the symptoms of a patient correctly so that they are handled specifically, therefore the patient should be placed within the correct group. For example, patients sharing the same symptoms from a particular disease can be put in a labelled group and those without these symptoms can be placed in another group, this way a degree of similarity can be identified.

To draw inferences the rational grouping of objects is required for various purposes not just in healthcare but in other areas for example marketing, biology, image segmentation and engineering, some of these application areas are discussed later.

Approaches to clustering vastly vary, a multitude of techniques have been proposed over the years and one of the reasons for this is that there is no such precise definition to the notion of "cluster" [161]

Clustering approaches can be divided into two different groups: hierarchical and partitioning techniques. Yet Han and Kamber [162]suggest further categories for applying clustering techniques: density-based methods, model-based methods and grid-based methods. Another categorisation based on the induction principle of different clustering approaches is presented in Castro et al [163]. More recently, according to Afrida [164], these conventional clustering approaches are not very effective in dealing with clusters which have 'overlapping regions' and propose three-way clustering (3WC) as being an effective and promising approach. Alternatively, many researchers of late take a 'combined methods' approach such as in image segmentation for example where a hybrid image segmentation method is used, this is based on a clustering algorithm and black hole algorithm [165]. Other approaches such as 'Multiobjective clustering' have emerged where multiple objective functions are simultaneously optimised and deemed as a robust alternative in such situations [166].

One of the biggest problems in the clustering community is deciding the optimal number of clusters, (discussed later in this Chapter) this means that prior to the clustering process the number of clusters into which available dataset is to be divided must be decided upon. This is carefully decided by the users, and approached differently by using methods such as heuristic, trial and error or evolutionary. High accuracy is the aim judged by intra-cluster distance, if of course the user decides an appropriate number. A good overview of the most common clustering techniques[167] is shown Figure 5.1 Clustering Techniques. 


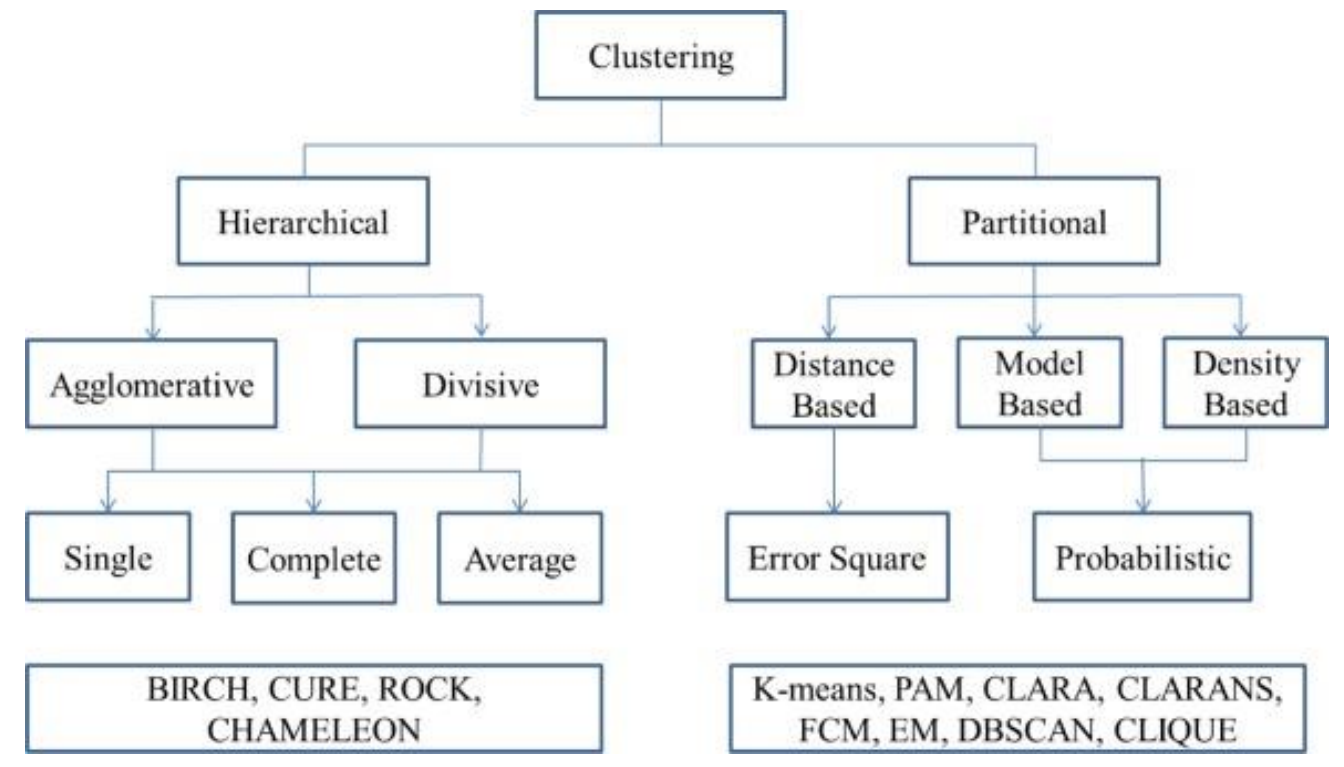

Figure 5.1 Clustering Techniques

Clustering analysis uses 'similarity measures' such as Euclidean-based distance or Correlation-based distance. These measures try to find homogeneous subgroups within the data such that data points in each cluster are as similar as possible. The decision on which 'similarity measure' to use is usually 'application-specific' [160].

Clustering is a division of data into groups of similar objects. Representing the data by fewer clusters necessarily loses certain fine details but achieves simplification. It models data by its clusters. Data modelling puts clustering in a historical perspective rooted in mathematics, statistics, and numerical analysis. From a machine learning perspective clusters correspond to hidden patterns, the search for clusters is unsupervised learning, and the resulting system represents a data concept. From a practical perspective clustering plays an outstanding role in data mining applications such as scientific data exploration, information retrieval and text mining, spatial database applications, Web analysis, CRM, marketing, medical diagnostics, computational biology, and many others [168].

As mentioned earlier traditionally clustering techniques are generally divided in hierarchical and partitioning [160]. Hierarchical clustering is further subdivided into agglomerative and divisive. The basics of hierarchical clustering include Lancewilliams formula, idea of conceptual clustering, now classic algorithms SLINK, COBWEB, as well as newer algorithms CURE and CHAMELEON [169].

While hierarchical algorithms build clusters gradually (as clusters are grown), partitioning algorithms learn clusters directly [170]. One such partitioning algorithm is K-means Clustering, which tries to discover clusters by iteratively relocating points between subsets or tries to identify clusters as areas highly populated with data. K-means clustering is also an unsupervised learning method which is a type of machine learning algorithm used to draw inferences from datasets consisting of input data without labelled responses [171]. 
In 2015 Gulati et al [172] did a large study surveying different clustering algorithms, snapshot is in Table 5.1. Analysis on Clustering [172] they concluded that when the clusters are of convex shape having similar size and the number of clusters can be identified prior Partitional clustering algorithms are the most useful. Yet sometimes due to the incapacity in foreseeing the number of clusters in advance Hierarchical clustering algorithms should be used. Although since Hierarchical clustering algorithms divide the dataset into several levels of partitioning called dendrograms, the cost of development of dendrograms is very high for bigger datasets. Also, observed is that when mining large datasets Density based clustering techniques are very useful [172]. The table identifies the type of dataset considered suitable for each algorithm, notably K-means suitability for numerical data whereas PFCM (Possibilistic Fuzzy C-Means) is best for Fuzzy and homogeneous data. Advantages and disadvantages for each algorithm assess various factors such as privacy and security and some such as K-means Parallel are noted for lack of support for heterogeneous (or diverse) datasets or MDCA (Multi-Hop Data Communication Algorithm) recognised as being useful in a non uniform schema.

According to Shreyansh Gokhru [173] the one and the most basic difference regarding where to use K-means (Partitioning) and Hierarchical clustering is on the basis of 'scalability' and 'flexibility'. Hierarchical is flexible but cannot be used on large data. K-means is scalable but cannot use for flexible data.

This is further evidenced as weakness in the fact that; agglomerative clustering methods do not scale well [174], also unfortunately with hierarchical clustering once a step (merge or split) is done, it can never be undone [175].

Yet observed in favour of the 'Hierarchical' algorithm it allows less assumptions about the distribution of data and it will provide a hierarchy, this structure being more informative than the unstructured flat clusters provided by K-means [176].

On the other hand, accuracy indicators often lead to the conclusion that K-means is more accurate than Hierarchical, for example in one study on an iris dataset it was found that the accuracy of K-means having "real" attributes was higher than the hierarchical clustering [177]. 
Table 5.1. Analysis on Clustering [172]

\begin{tabular}{|c|c|c|c|c|c|}
\hline Authors & Algorithm & $\begin{array}{l}\text { Clustering } \\
\text { Category }\end{array}$ & Dataset & Advantages & Disadvantages \\
\hline \begin{tabular}{|l} 
Dalal, \\
Harale \\
$(2011)$
\end{tabular} & $\begin{array}{l}\text { k- Means } \\
\text { Clustering }\end{array}$ & $\begin{array}{l}\text { Partitioning } \\
\text { Method }\end{array}$ & $\begin{array}{l}\text { Numerical data } \\
\text { (crisp data set) }\end{array}$ & $\begin{array}{l}\text { Large datasets } \\
\text { are processed } \\
\text { easily, Simple to } \\
\text { implement and } \\
\text { results are easy } \\
\text { to interpret }\end{array}$ & \begin{tabular}{|l} 
Sensitive to \\
noise, \\
Depends on \\
initial value of \\
k, poor locally \\
optimal \\
solutions
\end{tabular} \\
\hline $\begin{array}{l}\text { Dhillon, } \\
\text { Modha } \\
\text { (2002) }\end{array}$ & $\begin{array}{l}\text { K- Means } \\
\text { Parallel } \\
\text { Implementation } \\
\text { Partitioning } \\
\text { Method }\end{array}$ & \begin{tabular}{|l|} 
K- Means \\
Parallel \\
Implementation \\
Partitioning \\
Method
\end{tabular} & $\begin{array}{l}\text { crisp and } \\
\text { homogeneous } \\
\text { datasets }\end{array}$ & $\begin{array}{l}\text { Computation } \\
\text { time is reduced } \\
\text { by a factor of } \\
\text { p(no. of } \\
\text { processors) }\end{array}$ & $\begin{array}{l}\text { Do not support } \\
\text { heterogeneous } \\
\text { datasets }\end{array}$ \\
\hline $\begin{array}{l}\text { Vidya, } \\
\text { Clifton } \\
\text { (2003) }\end{array}$ & $\begin{array}{l}\text { Privacy } \\
\text { preserving } \\
\text { Kmeans } \\
\text { clustering over } \\
\text { vertical } \\
\text { partitioning } \\
\text { data }\end{array}$ & $\begin{array}{l}\text { Partitioning } \\
\text { Method }\end{array}$ & Crisp data sets & $\begin{array}{l}\text { Privacy and } \\
\text { security }\end{array}$ & $\begin{array}{l}\text { Do not support } \\
\text { heterogeneous } \\
\text { datasets }\end{array}$ \\
\hline $\begin{array}{l}\text { Moore, Hall } \\
(2004)\end{array}$ & DCA & $\begin{array}{l}\text { Partitioning } \\
\text { Method }\end{array}$ & $\begin{array}{l}\text { Both crisp and } \\
\text { fuzzy dataset }\end{array}$ & $\begin{array}{l}\text { Random } \\
\text { division and } \\
\text { distribution of } \\
\text { data set to local } \\
\text { site }\end{array}$ & $\begin{array}{l}\text { Complex due } \\
\text { to the } \\
\text { collision in } \\
\text { centroid } \\
\text { mapping }\end{array}$ \\
\hline $\begin{array}{l}\text { Visalakshi, } \\
\text { Thangavel, } \\
\text { Parvathi } \\
(2008)\end{array}$ & MDCA & $\begin{array}{l}\text { Partitioning } \\
\text { Method }\end{array}$ & $\begin{array}{l}\text { Crisp and } \\
\text { heterogeneous } \\
\text { dataset }\end{array}$ & $\begin{array}{l}\text { Useful in non } \\
\text { uniform schema }\end{array}$ & \\
\hline $\begin{array}{l}\text { Coletta, } \\
\text { Vendramin, } \\
\text { Hruschka, } \\
\text { Pedrycz } \\
(2012)\end{array}$ & PFCM & Fuzzy Method & $\begin{array}{l}\text { Fuzzy and } \\
\text { homogeneous } \\
\text { dataset }\end{array}$ & $\begin{array}{l}\text { Overlapping } \\
\text { clusters are } \\
\text { formed due to } \\
\text { usage of } \\
\text { membership } \\
\text { function, } \\
\text { Number } \\
\text { of clusters are } \\
\text { not to be } \\
\text { defined prior }\end{array}$ & $\begin{array}{l}\text { Data is of } \\
\text { similar type } \\
\text { i.e. } \\
\text { homogeneous }\end{array}$ \\
\hline $\begin{array}{l}\text { Ghanem, } \\
\text { Kechadi, } \\
\text { Tari } \\
(2011)\end{array}$ & IFDFC & Fuzzy Method & $\begin{array}{l}\text { Fuzzy and } \\
\text { homogeneous } \\
\text { dataset }\end{array}$ & $\begin{array}{l}\text { Privacy and easy } \\
\text { to use and } \\
\text { implement }\end{array}$ & $\begin{array}{l}\text { Homogeneous } \\
\text { datasets }\end{array}$ \\
\hline $\begin{array}{l}\text { Xu, Jager, } \\
\text { Kriegel } \\
(1999)\end{array}$ & $\begin{array}{l}\text { Parallel } \\
\text { DBSCAN }\end{array}$ & $\begin{array}{l}\text { Density based } \\
\text { method }\end{array}$ & $\begin{array}{l}\text { Crisp and } \\
\text { heterogeneous } \\
\text { data sets }\end{array}$ & \begin{tabular}{|l|} 
Excellent \\
scalability and \\
noise \\
is differentiated \\
efficiently
\end{tabular} & \\
\hline $\begin{array}{l}\text { Januzaj, } \\
\text { Kriegel, } \\
\text { Pfeifle } \\
(2004)\end{array}$ & SDBDC & $\begin{array}{l}\text { Density based } \\
\text { method }\end{array}$ & $\begin{array}{l}\text { Crisp and } \\
\text { Heterogeneous } \\
\text { data sets }\end{array}$ & Privacy & $\begin{array}{l}\text { Threshold } \\
\text { identification } \\
\text { is } \\
\text { Difficult }\end{array}$ \\
\hline
\end{tabular}




\section{C1.3 Clustering Application Areas and Selecting Clustering Approach}

Clustering analysis has been successfully executed in various fields, its use has been well documented and the different clustering techniques available have been carefully selected to apply to each problem situation. One such field is 'urban development' whereby decisions on how to place stations for setting up of industrial towns and economic hubs along highways [178] where made, here K-means clustering based on Ant Clustering Method was known to play a decisive role. The GFDBSCAN Clustering algorithm [179] was used to aid geographic positions for setting up amenities such as banks and schools. K-means was also implemented to identify patterns in online cable-discharge monitoring [180].

Clustering techniques have been performed extensively in the areas of computer security. It has been evidenced that using data from clustering can restrict cyber-criminals and thus enhance privacy protection. By partitioning data into clusters and providing a just a single relevant cluster to any information seeker responsible data sharing can be attained. This can be done using k-means algorithm [181] and distributed clustering [182].

In studying various aspects of human anatomy with the aim to understand the effects caused by a certain ailment, medical imaging has played a major role in medical science to treat patients. For example 'mean shift clustering' has been used in blood oxygen level dependent functional MRI activation detection [183]. Brain image segmentation has been done using semisupervised clustering [184] inhomogeneous medical image segmentation has been executed successfully using a hybrid method based on fuzzy clustering [185].

Additional fields where clustering has been utilised can be seen in a study by Ghosal, Nandy et al [186] in Table 5.2 Areas of Application and Algorithm used, this comprehensive table also identifies the clustering algorithm used in each sector and demonstrates the sheer diversity of techniques and how different domains have optimised a technique based on its helpfulness. Certain parameters are used to help evaluate and decide which technique to use. Some users require only an unsupervised grouping of objects into a number of clusters, where they can manually define the $\mathrm{K}$, so for this proper alignment then only choosing the value of $\mathrm{k}$ matters. This decision can be made based upon how accurate the intra-cluster objects (or patterns) by virtue of distance is expected from the user [187] and choosing $\mathrm{k}$ can be heuristic or stochastic and evolutionary computing like genetic algorithms (GA) can be applied to find $\mathrm{k}$. 
Table 5.2 Areas of Application and Algorithm used [186]

\begin{tabular}{|c|c|}
\hline Area of Application & Algorithm Used \\
\hline \multicolumn{2}{|l|}{ 1. In Banking: } \\
\hline $\begin{array}{l}\text { - Anti Money Laundering } \\
\text { Regulatory System }\end{array}$ & DBSCAN \\
\hline $\begin{array}{l}\text { - Broadcasting warning messages } \\
\text { against bank frauds }\end{array}$ & K-means, K-means++ \\
\hline $\begin{array}{l}\text { - Installing ATMs and e-corners at } \\
\text { strategic locations }\end{array}$ & DBSCAN \\
\hline $\begin{array}{l}\text { - Long-term bank failure } \\
\text { prediction }\end{array}$ & $\begin{array}{l}\text { Fuzzy Refinement domain } \\
\text { adaptation }\end{array}$ \\
\hline $\begin{array}{l}\text { - Profit maximization and } \\
\text { clientele expansion, customer } \\
\text { churn management }\end{array}$ & $\begin{array}{l}\text { Subtractive Clustering } \\
\text { Method(SCM) Fuzzy c- } \\
\text { means(FCM) }\end{array}$ \\
\hline \multicolumn{2}{|c|}{$\begin{array}{l}\text { 2. In segmentation engines and recommendation } \\
\text { engines: }\end{array}$} \\
\hline - Market analysis & K-means \\
\hline - Tourist market segmentation & Fuzzy C-medoids \\
\hline - Education & $\begin{array}{l}\text { K-means and Hierarchical } \\
\text { clustering }\end{array}$ \\
\hline - Crime domain documentation & K-means, k-means++ \\
\hline $\begin{array}{l}\text { - Drawing patterns in social media } \\
\text { usage handling all the side- } \\
\text { information and metadata within } \\
\text { any document working of web } \\
\text { search engines }\end{array}$ & $\begin{array}{l}\text { k-means, fuzzy C-means and } \\
\text { hierarchical clustering algorithms }\end{array}$ \\
\hline \multicolumn{2}{|l|}{ 3. In Health Care: } \\
\hline $\begin{array}{l}\text { - Detection of } \\
\text { neurovascularization in retinal } \\
\text { images }\end{array}$ & $\begin{array}{l}\text { Multivariate m-Medoids based } \\
\text { classifier }\end{array}$ \\
\hline - Detection of tumors & K-means \\
\hline $\begin{array}{l}\text { - Blood oxygen level dependent } \\
\text { functional MRI activation } \\
\text { detection }\end{array}$ & Mean shift algorithm \\
\hline - Brain image segmentation & Spectral clustering \\
\hline $\begin{array}{l}\text { - Inhomogeneous medical image } \\
\text { segmentation }\end{array}$ & Fuzzy clustering \\
\hline - Medical image analysis & Spectral clustering \\
\hline \multicolumn{2}{|l|}{ 4. In Urban Development: } \\
\hline $\begin{array}{l}\text { - Positioning of towns, building } \\
\text { efficient power supplies }\end{array}$ & $\begin{array}{l}\text { K-means clustering based on Ant } \\
\text { Clustering Method }\end{array}$ \\
\hline $\begin{array}{l}\text { - Setting up amenities such as } \\
\text { banks, schools etc }\end{array}$ & DBSCAN \\
\hline - Transformer fault diagnosis & k-medoids \\
\hline \multicolumn{2}{|l|}{$\begin{array}{l}\text { - Identifying patterns in online } \\
\text { cable-discharge }\end{array}$} \\
\hline - Transformer fault diagnosis & k-medoids \\
\hline $\begin{array}{l}\text { - Identifying patterns in online } \\
\text { cable-discharge Monitoring }\end{array}$ & k-means \\
\hline $\begin{array}{l}\text { - Population estimation with } \\
\text { satellite imagery }\end{array}$ & Expectation Maximization \\
\hline
\end{tabular}

Despite the 'choosing k' challenge k-means clustering dominates and is still the most popular clustering method. In addition to Table 5.1. Analysis on Clustering [172] which previously identified useful factors to help a user choose an algorithm, a few other researchers have performed studies undertaking the task of deciding a clustering method, for example Fraley [167] describe a clustering methodology based on multivariate normal mixture models and showed it can give much better performance than existing methods, but Saxena et al. [187] 
found many limitations with this such that computational methods for hierarchical clustering cannot be directly applied to large data sets since they have storage and time requirements that grow at a faster than linear rate relative to the size of the initial partition. Bensmail et al. [188] showed that exact Bayesian inference via Gibbs sampling, with calculations of Bayes factors using the Laplace-Metropolis estimator, works well in several real and simulated examples [167].

Further parameters used to evaluate a clustering techniques merit or usefulness depending on a user's purpose are presented in Table 5.3 Parameters to Evaluate Clustering Techniques. For example, for large data sets, CURE method is advisable unlike ROCK but with less time complexity although scalability is high to that obtained by CURE. This table is a useful source measuring each technique by parameters such as time complexity, scalability, large scale data suitability, high dimensional data suitability and noise sensitivity.

Table 5.3 Parameters to Evaluate Clustering Techniques

\begin{tabular}{|c|c|c|c|c|c|c|}
\hline $\begin{array}{l}\text { Category of } \\
\text { Clustering }\end{array}$ & $\begin{array}{l}\text { Algorithm } \\
\text { Name }\end{array}$ & Time complexity & Scalability & $\begin{array}{l}\text { Suitable for } \\
\text { large scale data }\end{array}$ & $\begin{array}{l}\text { Suitable for } \\
\text { high } \\
\text { dimensional } \\
\text { data }\end{array}$ & $\begin{array}{c}\text { Sensitive } \\
\text { of noise/ } \\
\text { outlier }\end{array}$ \\
\hline \multirow[t]{4}{*}{ Partition } & k-means & Low O(knt) & Middle & Yes & No & High \\
\hline & PAM & High $\left.\mathrm{O}\left(\mathrm{k}(\mathrm{n}-\mathrm{k})^{\wedge} 2\right)\right)$ & Low & No & No & little \\
\hline & CLARA & Middle $\mathrm{O}(\mathrm{ks} \wedge 2+\mathrm{k}(\mathrm{n}-\mathrm{k}))$ & High & Yes & No & Little \\
\hline & CLARANS & High $\mathrm{O}\left(\mathrm{n}^{\wedge} 2\right)$ & Middle & Yes & No & Little \\
\hline \multirow[t]{4}{*}{ Hierarchy } & $\mathrm{BIRCH}$ & Low $\mathrm{O}(\mathrm{n})$ & High & Yes & No & Little \\
\hline & CURE & Low O(s ^2*logs) & High & Yes & Yes & Little \\
\hline & ROCK & High $\mathrm{O}\left(\mathrm{n}^{\wedge} 2 * \log n\right)$ & Middle & No & Yes & Little \\
\hline & Chameleon & High $\mathrm{O}\left(\mathrm{n}^{\wedge} 2\right)$ & High & No & No & Little \\
\hline Fuzzy based & FCM & Low $\mathrm{O}(\mathrm{n})$ & Middle & No & No & High \\
\hline Density based & DBSCAN & Middle $O(n * \log n)$ & Middle & Yes & No & Little \\
\hline Graph theory & CLICK & Low $\mathrm{O}(\mathrm{k} * \mathrm{f}(\mathrm{v}, \mathrm{e}))$ & High & Yes & No & High \\
\hline Grid based & CLIQUE & Low $\mathrm{O}\left(\mathrm{n}+\mathrm{k}^{\wedge} 2\right)$ & High & No & Yes & Moderate \\
\hline
\end{tabular}

The application of clustering is wide, the strengths and limitations of each technique are available within the vast amount of literature. Deciding which technique to use can be determined by evaluating parameters and keeping in mind the user's overall purpose. Objectively this section has presented a rationale to demonstrate it is not feasible to agree on one recommended clustering technique. A user's objective and strategy will be different, and each should be worked through experimentally. 


\section{C1.4 Selected Technique K-means}

Since finding the maximum similarity among epilepsy patient profile groups is the goal a clustering technique principally to measure similarity of objects within a cluster, would be the most appropriate technique to use.

Due to the aim of accuracy in results and the fact that the size of the ESO dataset is expected to grow to large, the K-means method is chosen for this study. Also, since K-means clustering has commonly known use in medical fields and is one of the simplest clustering technique. [189]. Some of the other available techniques such as PFCM (Possibilistic Fuzzy C-Means) although good for complex data have shown overlapping clusters due to usage of membership function and since the number of clusters are not to be defined prior [172] limits control. Saleability for K-means technique is judged as 'middle', whereas the CLARA technique is 'high' (according to Table 5.3 Parameters to Evaluate Clustering Techniques) this is apt at this stage since it will be a fair applicability to large datasets and performance is expected to decrease linearly with data size increase.

K-means uses unsupervised learning method to solve known clustering issues. Another reason that K-means is chosen is that it works well with heterogeneous datasets, such as the dataset used for ESO in this study, since it contains many attributes, this will be useful as observed by Trevino, in identifying unknown groups in complex data sets [190].

Moreover, since there is no prior knowledge of groups with the ESO patient profile dataset it is decided to use this 'Unsupervised method' for this study, as presently the dataset only contains features and instances so K-means will be used to group them. The basic step of Kmeans clustering is simple. In the beginning, the number of cluster $\mathrm{K}$ is determined and the centroid or center of these clusters is assumed, see Figure 5.2. K-means Clustering Process [142].

Any of the random objects as the initial centroids is taken or the first K objects can also serve as the initial centroids. Then the K-means algorithm will do the three steps below until convergence. Iterate until stable (= no object move group):

1. Determine the centroid coordinate

2. Determine the distance of each object to the centroids

3. Group the object based on minimum distance (find the closest centroid). 


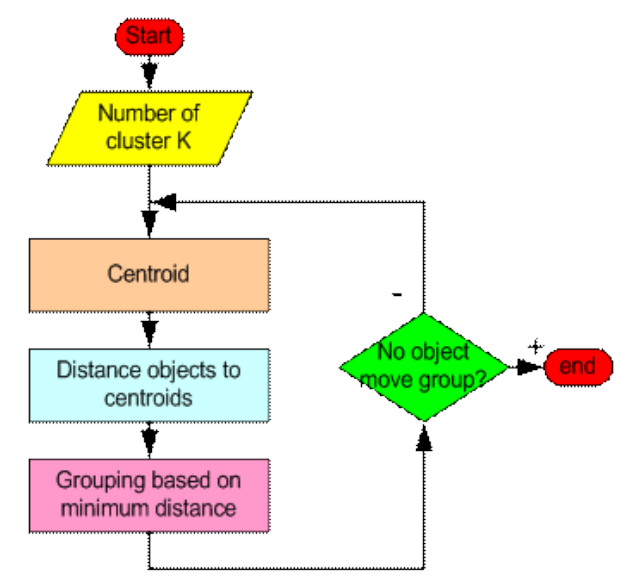

Figure 5.2. K-means Clustering Process

The K-means method uses Euclidean Distance, which is the most common distance measurement used in clustering. Furthermore this is deemed as one of the most apparent ways to represent distance between two points [191].

Euclidean distance or simply 'distance' examines the root of square differences between coordinates of a pair of objects [192] In basic terms this distance measure calculates the distance between two points [193]. The formula below shows the square root of the sum of the distance $(d)$ of the points from each other, squared. It can also be described as a distance measure between a pair of points $x$ and $y$ in an n-dimensional feature space (or variables) with case $i$. Therefore given $2 n$-dimensional variable's $x$ and $y$ :

$$
\begin{aligned}
& -x=\left(x_{1}, x_{2}, \ldots x_{n}\right) \\
& -y=\left(y_{1}, y_{2}, \ldots y_{n}\right)
\end{aligned}
$$

Euclidean distance $d(x, y)$ is defined as the formula [194] in Equation 1. ED Formula below:

$$
d(x, y)=\sqrt{\sum_{i=1}^{n}\left(x_{i}-y_{i}\right)^{2}}
$$

Equation 1. ED Formula

For example: Point $x$ has coordinate $(3,0,1)$ and point $y$ has coordinate $(5,2,0)$.

I.e. $\quad \boldsymbol{X}=(3,0,1) \quad \boldsymbol{y}=(5,2,0)$

The Euclidean Distance between point $x$ and $y$ is: (see Equation 2. ED '[194] below) equating to the root of square differences between coordinates of a pair of objects. 


$$
\text { - } \mathbf{D}(\mathbf{x}, \mathbf{y})=\sqrt{(3-5)^{2}+(0-2)^{2}}+(1-0)^{2}=3
$$

Equation 2. ED Answer

To demonstrate simply Euclidean distance formula can be used to calculate the distance between two data points in a plane, see Figure 5.3. Euclidean Distance [194]

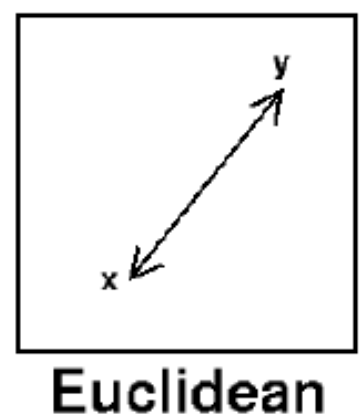

Figure 5.3. Euclidean Distance

Euclidean Distance is one of many similarity distance measures, Manhattan Distance being another popular measure. Since this study is looking for attributes that are similar (so being in the same space or neighbourhood) then Euclidean distance is the most suitable. Other similarity measures exist such as Correlation-based distance, which is widely used for gene expression data analyses [195] but these focus on things that go up and down together, regardless of their range. From a good distance measure, it is expected that there is a high degree of accuracy and robustness. Euclidean Distance has its criticisms but is widely endorsed and commonly used by several other authors in similar studies whereby its function can produce significant robustness [196] and a study in transportation services measuring the accuracy of EDC confirmed that Euclidean distance helped them to achieve an excellent level of accuracy [197].

\section{C2. Perform Clustering}

The next stage outlines the methods used to performing Clustering using the K-means algorithm. The following sections includes data preparation and pre-processing and data cleaning.

\section{C2.1 Dataset Preparation \& Pre-processing}

One of the important steps of data mining process is data pre-processing. Data pre-processing is used in identifying the missing values, noisy data and irrelevant and redundant information from dataset [198]. At this stage the data set is prepared. 
The dataset was prepared in the Ontology Stage. Yet some re-arranging and further analysis/editing is needed in to ensure it is correct and worthy for the intended OBJ 3: 'Perform a Clustering technique to discover distinct groups of patients that share similar characteristics.'

It is discovered that the original dataset is wide-ranging and has 60 signs and symptoms collected from the patient medical records, a snapshot is shown below in Table 5.4:

Table 5.4 Signs \& Symptoms Snapshot

\begin{tabular}{|c|c|}
\hline Palpitations & Dizziness \\
\hline Clonus & Twitching \\
\hline Stiffening & Bilateral_shaking \\
\hline Stiffening_rightarm & Hyperkinetic \\
\hline Stiffening_leftarm & Sensations \\
\hline Stiffening_rightleg & Tongue biting \\
\hline Stiffening_leftleg & Visual \\
\hline Stiffening_upperlimbs & Urinary \\
\hline Stiffening_lowerlimbs & Déjà_Vu \\
\hline Vacant & Lip_smacking \\
\hline Cyanosis & Fingers \\
\hline Convulsions & Dissociation \\
\hline Loss_of_consciousness & Emotional \\
\hline Nausea & Incontinence \\
\hline Clonus_right & Vocalisation \\
\hline Clonus_left & Eyelid_myoclonia \\
\hline
\end{tabular}




\begin{tabular}{|l|l|}
\hline Clonus_upperlimb & Menstrual_cycle \\
\hline Clonus_lowerlimb & Walking \\
\hline Aphasia & Fall \\
\hline Stress & Awareness \\
\hline Sleep_dep & Rightarm_sensation \\
\hline Vomit & Head deviation \\
\hline
\end{tabular}

According to Robert Fisher [150] the Key Signs and Symptoms for seizures can be put into the following categories listed, together with their updated 'Medical Term' in Table 5.5:

Table 5.5. Key Signs and Symptoms

Symptoms

Automatic behaviors

Emotions or appearance of emotions

Extension or flexion postures

Flushing/sweating/piloerection

Jerking arrhythmically

Jerking rhythmically

Language or thinking

problems, Deja vu

Lid jerks

$\operatorname{Limp}$
Medical

Term

Emotions

Tonic

Autonomic

Myoclonus

Clonus

Cognitive

Eyelid myoclonia

Atonic 
Numb/tingling, sounds, smells,

tastes visions, vertigo

Pausing, freezing, activity

arrest

Thrashing/pedaling

Trunk flexion
Sensations

Behavior arrest

Hyperkinetic

Spasm

In addition to Table 5.5 it is identified in the Ontology Chapter in "Categorising Routinely Used Terms" that other signs and symptoms can be put under the following categories shown in Table 5.6.

Table 5.6. Categorising Routinely used Terms

\begin{tabular}{|l|l|}
\hline Cognitive & $\underline{\text { Automatisms }}$ \\
Acalculia & Aggression \\
Aphasia & Eye-blinking \\
Attention impairment & Head-nodding \\
Déjà vu or jamais vu & Manual \\
Dissociation & Oral-facial \\
Illusions & Pedalling \\
Memory impairment & Pelvic thrusting \\
Arying (dacrystic) & Perseveration \\
Forced thinking & Running (cursive) \\
Responsiveness impairment & Sexual \\
\hline
\end{tabular}




\begin{tabular}{|c|c|}
\hline Fear & Fencers posture \\
\hline Laughing & Incoordination \\
\hline Paranoia & Jacksonian \\
\hline \multirow[t]{2}{*}{ Pleasure } & Paralysis \\
\hline & Paresis \\
\hline Autonomic & Versive \\
\hline Asystole & \\
\hline Bradycardia & $\underline{\text { Sensory }}$ \\
\hline Erection & Auditory \\
\hline Flushing & Gustatory \\
\hline Gastrointestinal & Hot-cold sensations \\
\hline Hyper/hypoventilation & Olfactory \\
\hline Nausea or vomiting & Somatosensory \\
\hline Pallor & Visual \\
\hline Palpitations & \\
\hline Piloerection & Laterality \\
\hline Respiratory changes & Left \\
\hline Tachycardia & Right \\
\hline & Bilateral \\
\hline
\end{tabular}

Adjudicating from the occurrences of signs and symptoms in the patient medical records it is apparent that those in Table 5.6 are indeed less common than those identified in Table 5.5. This is a good way to organise and re-arrange the dataset in preparation for clustering, by narrowing and refining the data, whereby signs and symptoms are mapped to new categories, a snapshot is shown in Figure 5.4. Curating Epilepsy Terms, (the colouring scheme shown in Figure 5.4 is described below).

The new categories are named 'Key Signs and Symptoms' are shown in green and 'Common Signs and Symptoms' in pink. During further analysis 'New Categories' (shown in blue) are discovered and added to 'Key Signs and Symptoms.' These other prominent Key Signs and Symptoms that frequently occur throughout the patient medical records are:

- $\quad$ LOC (Loss of Consciousness)

- Bilateral_Tonic (Both Left \& Right side) 
- Bilateral_Clonus (Both Left \& Right side)

- Bilateral_Shaking (Both Left \& Right side)

- Urinary/Incontinence

Some of the categories from Key Signs and Symptoms found in Table 5.5. Key Signs and Symptoms were also found in Table 5.6. Categorising Routinely used Terms. Therefore, these categories were deemed as redundant so where either removed or moved (see in grey in

Figure 5.4).

From the 100 patient medical records it is also observed that often the patient would have a trigger such as 'Menstrual Cycle' just before the seizure occurred, this way the patient would know that a seizure was imminent. Other common triggers where observed from the records including: 'Sleep deprivation', 'Stress' and 'Eating'. Therefore, these categories were added to the new dataset in preparation for Clustering. See in yellow in Figure 5.4

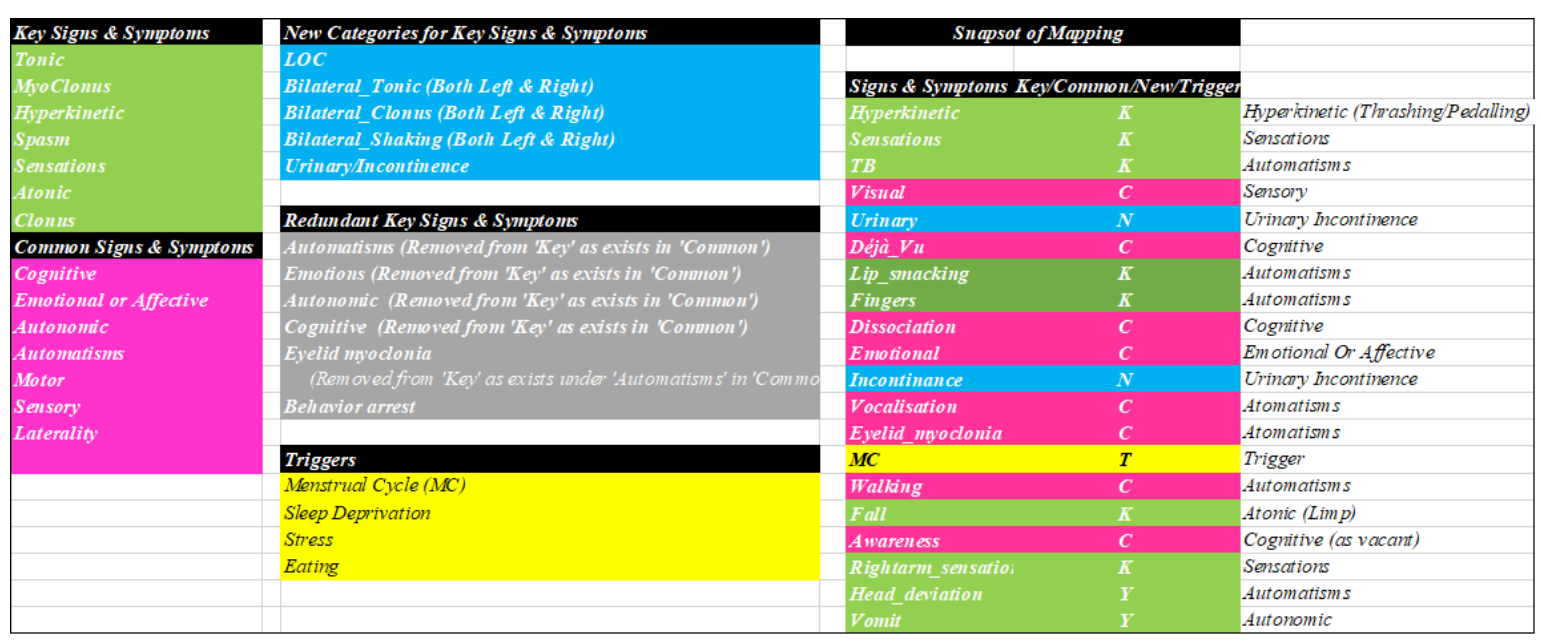

Figure 5.4. Curating Epilepsy Terms

Clinicians can advise the position on the body of the monitoring sensor, this can be determined by where the seizure begins on the patient (left arm/leg, right arm/leg) or which side the seizure mainly occurs. It is evident from the patient medical records that notes were recorded from observations about the 'dominant side' and 'limb factors' as witnessed in some profiles in Table 5.7. Seizure Initiation Factors. Therefore, the categories 'Arm' and 'Leg' are added together with 'Dominant Left' and 'Dominant Right' to the new dataset in preparation for Clustering. 
Table 5.7. Seizure Initiation Factors

\begin{tabular}{|c|c|c|c|}
\hline & & & Dominant Side \\
\hline $\begin{array}{l}\text { Profile } \\
95\end{array}$ & GTC & Stress, sleep dep, & $\begin{array}{l}\text { Right arm extends \& } \\
\text { clenches \& right leg } \\
\text { extend, drop to floor } \\
\text { (collapse) vocalise, } \\
\text { incontinence }\end{array}$ \\
\hline $\begin{array}{l}\text { Profile } \\
96\end{array}$ & $N M A$ & Feels Hot & $\begin{array}{l}\text { LOC, shaking, eyes open } \\
\text { (up to } 30 \text { secs) }\end{array}$ \\
\hline $\begin{array}{l}\text { Profile } \\
97\end{array}$ & GTC & $\begin{array}{l}\text { Illness, constipation, } \\
\text { asking for bed }\end{array}$ & $\begin{array}{l}\text { Vocalisation, left arm } \\
\text { extends, (stiffens), } \\
\text { shaking, tongue bite, lips } \\
\text { turning blue }\end{array}$ \\
\hline $\begin{array}{l}\text { Profile } \\
98\end{array}$ & $N M A$ & $\begin{array}{l}\text { Distorted perception } \\
\text { of environment } \\
\text { Feeling like on a ship } \\
\text { Blurred Vision (left } \\
\text { eye) }\end{array}$ & Dancing Feet \\
\hline $\begin{array}{l}\text { Profile } \\
99\end{array}$ & $\begin{array}{l}\text { GTC, } \\
\text { NMA } \\
\text { Gelastic }\end{array}$ & $\begin{array}{l}\text { Change in mood, } \\
\text { sleepy, excitement, } \\
\text { constipation, ill health }\end{array}$ & $\begin{array}{l}\text { Left arm \& left leg } \\
\text { extension \&shaking, } \\
\text { grunting, grabbing at } \\
\text { people \& objects, talking } \\
\text { then LOC ( } 3 \text { mins })\end{array}$ \\
\hline
\end{tabular}

\section{C2.2 Data Cleaning}

Most implementations of K-means only allow numerical values for attributes. To create meaningful clustering, all the non-numerical attributes are converted to the numeric attributes (without affecting the meaning of those attributes while deciding the cluster), as discovered $\mathrm{K}$-means cannot handle categorical variable directly and uses distance computation at core of its algorithm [150]. 
Therefore, a Boolean value '0,1' or 'True or False' is assigned as in Fig.45. Data Cleaning Sample. To begin the raw data is analysed and converted to binary: ' 0.1 ' then it is again converted to Boolean: 'True/False'. This way all categorical attributes are converted to binary values, and then K-means is performed as if these were numeric values.

As witnessed by Mobasher [199] it was found that it may also be required to normalise the values of attributes that are measured on significantly different scales (e.g., "age" and "income"). Therefore, upon analysing the dataset used in this study it is observed that some categories are on different scales compared to others; 'Seizure Types' and 'Seizure Occurrences'. Therefore coding/labelling systems are used to aid the process (see Figure 5.5. Data Cleaning Sample).

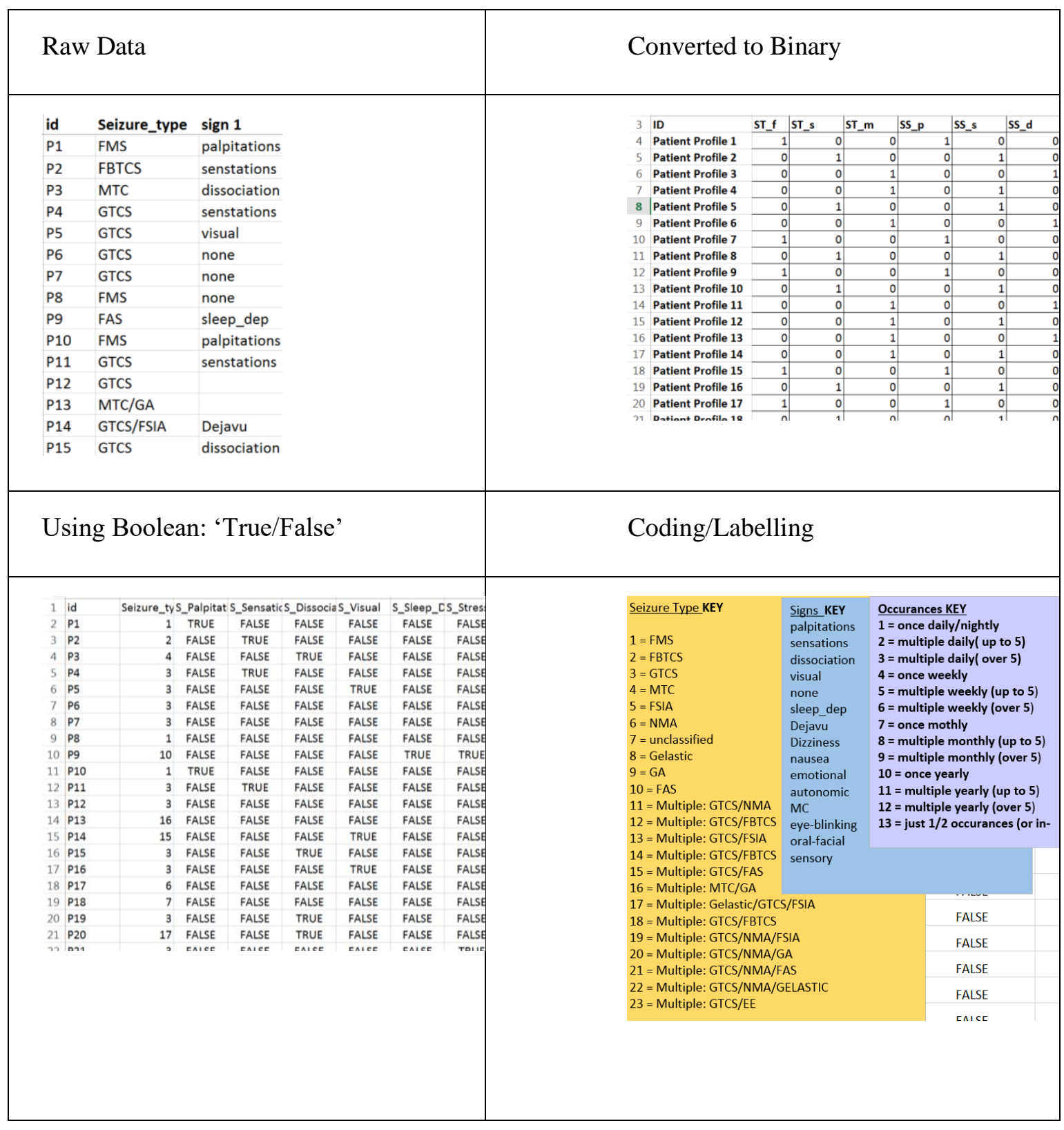

Figure 5.5. Data Cleaning Sample

Next the pre-processing is performed. The figure below demonstrates that some filtering was needed because the class attribute 'id' is providing an invalid signal that is overlapping the valid data creating redundant data as can be seen in 'Figure 5.6. With 'id' Attribute', whereby 
1 count (attribute) for each of the 100 patient profile is visible, which does not reveal anything. Therefore, the attribute for 'id' (Patient Profile) is removed as it is redundant.

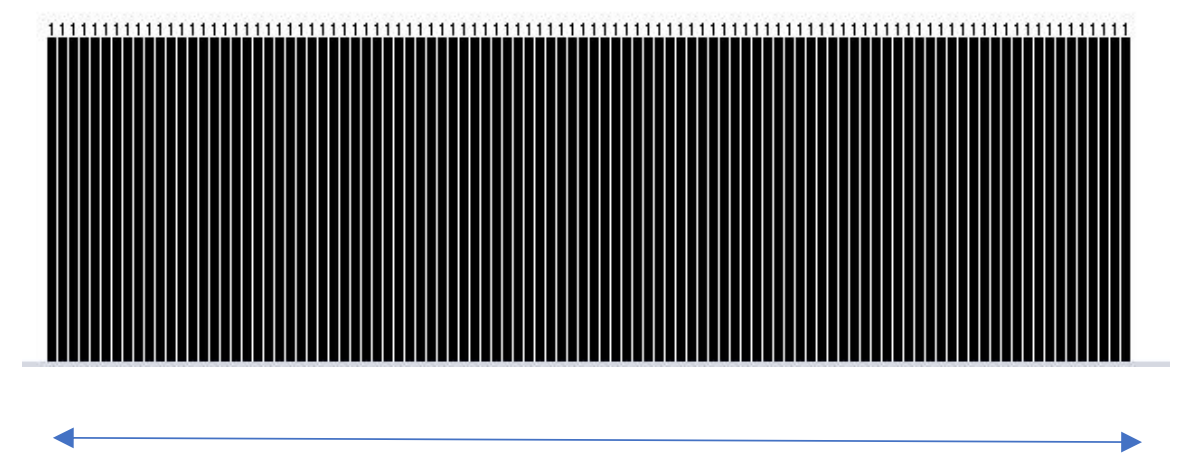

Patient Profiles 1-100

Figure 5.6. With 'id' Attribute

The correct types and values associated with the attributes are automatically determined as observed during further pre-processing, here 29 attributes and 100 instances are established from the dataset. When (for example) the 'DOMINANT LEFT' (Figure 5.7. Dominant Left) is selected some information about this attribute can be visualised. For example, 82/100 have the seizure occurring on the 'left' side and 18/100 do not. These two distinct values (true and false) are derived from the values in the dataset.

Whereas when 'Seizure Type' is selected (Figure 5.8. Seizure Type) there is a slightly different view as the values for this attribute are numeric, $7 \%$ are unique which means they cannot match with each other. This demonstrates, (from the 23 seizure types) how seizure types 1-4 are the most common with 41 . These Seizure types are FMS, FBTCS, GTCS and MTC.

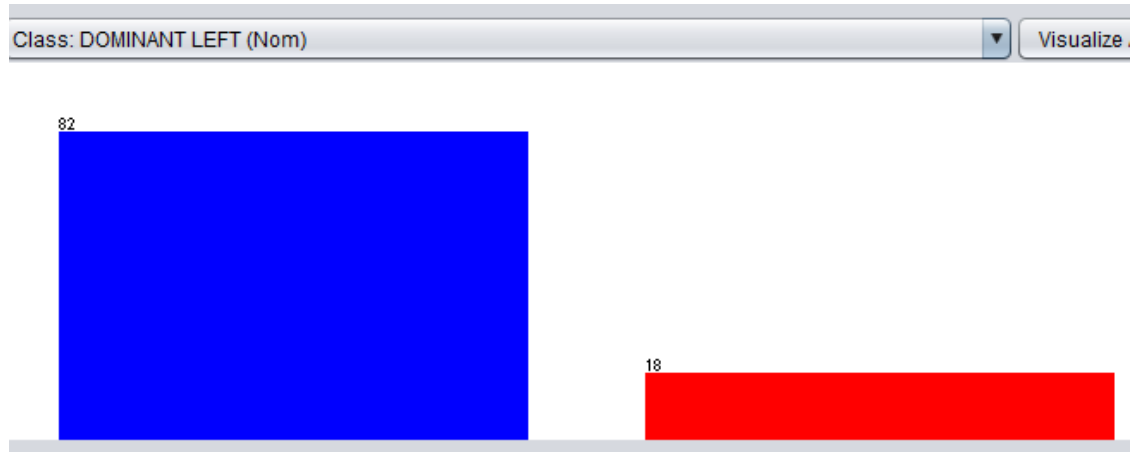

Figure 5.7. Dominant Left 


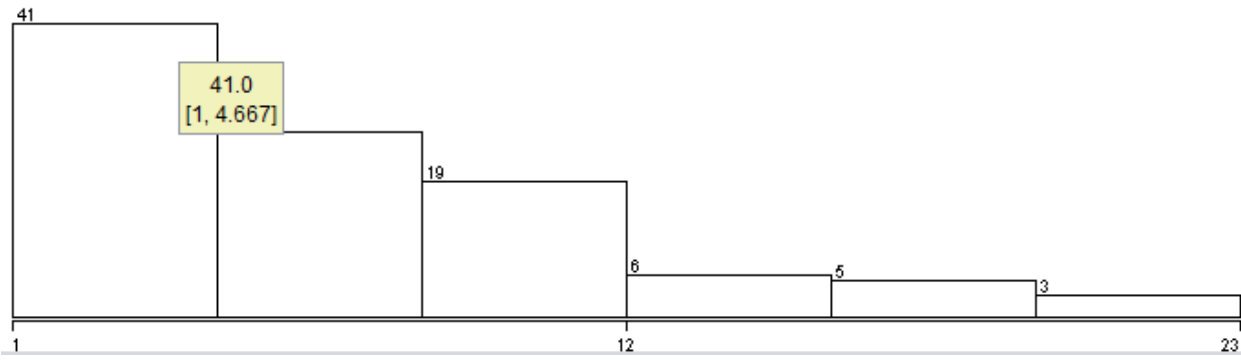

Seizure Types (1-23)

Figure 5.8. Seizure Type

\section{C2.3 Clustering Considerations}

The K-means is chosen as the clustering method from a list of clusters. The chosen numclusters is 6. This chosen number of clusters is discussed later in 5.5.3.

The seed number is next chosen (discussed below). Then the various cluster modes are considered i.e.: Use training set, Supplied test set, Percentage split or Classes to cluster evaluation. Percentage spilt is chosen as $66 \%$ then the clustering process begins.

\section{C2.4 Percentage Split}

The 'Percentage split 'option will train the classifier on the indicated percentage of the data and test it on the rest. As observed Browmlee [200] that a common split value is $66 \%$ to $34 \%$ for train and test sets respectively, but this should not to be used to make decisions, unless working with a very large dataset and it has been tested [200]. Yet, do the splits sufficiently describe the problem? A good reason why the percentage split should be used is witnessed by Cohen [201] whereby he ascertains that a percentage split should be used to avoid a problem called "over-fitting", whereby trends are learnt in training data that might not exist in the real world.

The percentage split (66\%) for training and $34 \%$ for testing is used for the evaluation mode in this study as it would not be good to learn a very specific set that occurs by chance in the training data, because it would not really reveal anything about the pattern, since the pattern might not be observed in other data. It is best to test on data that hasn't been seen before so that it's possible to simulate having real-world data to test the pattern on, rather than testing on the same data trained with. 


\section{C2.5 Choosing the Seed}

The data is shuffled randomly based on the random number seed. The seed can be altered from 1-10. This is done using the 'Fisher-Yates' shuffle in WEKA [130].

When the seed is altered different results are produced. K-means always uses random numbers, if the same seed always remains the same, the same random numbers will always be returned. Which means, if K-means is run over the same data twice, and if both times the same seed is used, the result will be the same. Whereas if same seed is not used, results will vary every time K-means is run [202].

Seed numbers are numbers only due to the way random number generators are implemented. Also observed is that different seeds produce slightly different error rates, the seed number used in this study is number 10, since this produced a low number of SSE (Sum of squared Errors), but further justification for this seed number is discussed in section 5.5.5.

\section{C2.6 Choosing the Optimal Number of Clusters}

As identified in the earlier sections of this chapter determining the initial number of clusters when performing unsupervised clustering is a complicated problem and can be heuristic or stochastic. There are various methods available for deciding the optimal value for " $\mathrm{k}$ " in $\mathrm{K}$ means algorithm including thumb-Rule, elbow method and silhouette method etc. Yet the ideal number of clusters is subjective [203]. As observed by Vincent Granville [204] upon questioning what the ideal number of clusters is, he conveyed that no one can tell with certainty, not AI, not a human being, not an algorithm. Even today people are still doing research. Consequently a heuristic approach is taken and the number of optimal clusters chosen for this study is 6 since this number of groups demonstrates a good sample/range to showcase what is achieved with clustering seizure-type patient profiles and henceforth there is no concrete agreement that would justify the search of the ideal number of clusters [205].

\section{C2.7 Evaluating K-means Results}

The Cluster results show the centroid of each cluster, thus, centroids can be used to characterize the clusters, the results show the statistics on the number and percentage of instances assigned to different clusters, each dimension value in the centroid represents the mean value for that dimension in the cluster. For example, the centroid for cluster group 2 shows that this segment of patients represents seizure type 5 seizure Type $10=$ FAS (Focal Aware Seizure, previously termed 'Auras') also the Key Signs \& Symptoms 'Sensation' and 'Automatisms' are 'TRUE', this group also only has seizures during 'Diurnal' (day-time), 
and seizures occur both on the 'Dominant Right' and 'Dominant Left'. Furthermore, this group has Seizure Occurrences ' 6 ' which is 'multiple weekly (over 5)', and no triggers recorded for their seizures.

Extracted from the Cluster Output the cluster groups 0-5 are displayed in Table 5.8. Cluster Groups. The 6 cluster groups reveal six completely different categories of patients each with their distinct seizure related information. 'Cluster Group 1' although reveals an 'unclassified' seizure type, this is just as relevant as those with identified seizure types since they reflect a 'real world' model of what one of the individual seizure type patients is like. 'Cluster Group 0 ' has seizures which are dominant on the right side of the body, occur over 5 times a week and either during daytime or night-time, they will have a loss of consciousness, together with urinary/incontinence and some automatisms (i.e. Tongue biting, finger rubbing, lip smacking etc.), this group specifies the neither arm or leg meaning that seizures could begin or effect any part of the body. 


\begin{tabular}{|c|c|c|c|c|c|c|}
\hline Attribute & Cluster & & & & & \\
\hline & Cluster 0 & Cluster 1 & Cluster 2 & Cluster 3 & Cluster 4 & Cluster 5 \\
\hline Seizure Type & $N M A$ & Unclassified & $F A S$ & Gelastic & GTCS & MTC \\
\hline \multirow[t]{2}{*}{ Key Sign \& Symptoms } & $\begin{array}{l}\text { LOC (Loss of } \\
\text { Consciousness) }\end{array}$ & $\angle O C$ & Sensations & None & $\begin{array}{l}\text { Myoclonus \& } \\
\text { Bilateral Clonus }\end{array}$ & Bilateral Clonus \\
\hline & $\begin{array}{l}\text { Urinary/ } \\
\text { Incontinence }\end{array}$ & None & & & $L O C$ & $L O C$ \\
\hline $\begin{array}{l}\text { Common Sign \& } \\
\text { Symptoms }\end{array}$ & Automatisms & $\begin{array}{l}\text { Cognitive } \\
\text { Automatisms }\end{array}$ & Automatisms & Automatisms & $\begin{array}{l}\text { Sensory } \\
\text { Cognitive }\end{array}$ & Automatisms \\
\hline Arm/Leg & Either & Leg & Either & Either & Leg & Either \\
\hline Nocturnal/Diurnal & $\begin{array}{l}\text { Nocturnal and } \\
\text { Diurnal }\end{array}$ & $\begin{array}{l}\text { Nocturnal and } \\
\text { Diurnal }\end{array}$ & Diurnal & Diurnal & $\begin{array}{l}\text { Nocturnal and } \\
\text { Diurnal }\end{array}$ & Diurnal \\
\hline
\end{tabular}




\begin{tabular}{|l|l|l|l|l|l|l|}
\hline Seizure Occurrences & $\begin{array}{l}\text { Multiple weekly (over } \\
\text { 5) }\end{array}$ & Once Monthly & $\begin{array}{l}\text { Multiple weekly } \\
\text { (over to 5) }\end{array}$ & $\begin{array}{l}\text { Multiple weekly } \\
\text { (over to 5) }\end{array}$ & $\begin{array}{l}\text { Multiple monthly } \\
\text { (up to 5) }\end{array}$ & $\begin{array}{l}\text { Multiple weekly } \\
\text { (up to 5) }\end{array}$ \\
\hline Dominant Side & Right & Left and Right & Left and Right & Left and Right & Right & Seft and Right \\
\hline Trigger & None & None & None & None & Sleep Dep \\
\hline
\end{tabular}




\section{C2.8 Sum of Squared Errors}

The SSE (Sum of squared errors) SSE is the "sum of the squared differences between each observation and its group's mean. It can be used as a measure of variation within a cluster. If all cases within a cluster are identical the SSE would then be equal to 0" [206]. There is much discussion about returning the Sum of Squared Errors in the cluster output and the important question here is: "does the lower amount of squared errors achieve a more accurate result?" It is easy to just pick a result with a specific seed value that has the lowest SSE, but this does not really hold any validation. Yet the goal of K-means is to reduce the sum of the squared error over all k clusters [207] [208]. One method to achieve this is if you increase the number of clusters you tend to get a lower SSE [209]. Again, this is easy, but a good clustering with smaller K can have a lower SSE than a poor clustering with higher K [160]. Therefore, this argument can link to 'how to choose the initial number of ' $\mathrm{K}$ '? (' $\mathrm{K}$ ' known as clusters); discussed in C.2.2.4. As with many algorithms the limitations are many, and as witnessed perhaps the answer appears to lie in some or all of the hidden patterns to ascertain whether the cluster output is actually meaningful [160]. Founded on this discussion the seed value 10 is chosen in this study as it produced the $2^{\text {nd }}$ lowest SSE: ' 188.07751 ', (out of 10) and represented a good variation of seizure types. Seed 6 produced a slightly lower rate at: '182.90444' yet the cluster group patterns for seed 6 did not include the most common seizure type being GTCS.

\section{C2.9 Visualising Results}

Another way of understanding the characteristics of each cluster is through visualization. The data just provides the centroid but with the visualisation tool deeper analysis is enabled.

This is shown in Figure 5.9. Visualising Cluster Assignments which shows the patients in clusters (coloured for each cluster) on the X-Axis. The Y-Axis shows the seizure type and it is evident that the clusters are sharing common seizure type 3 GTCS General Tonic Clonic Seizure which is true to data count in the data set. Based on 60 random patient profiles the 6 cluster assignments for the 23 different seizure types can be visualised. 


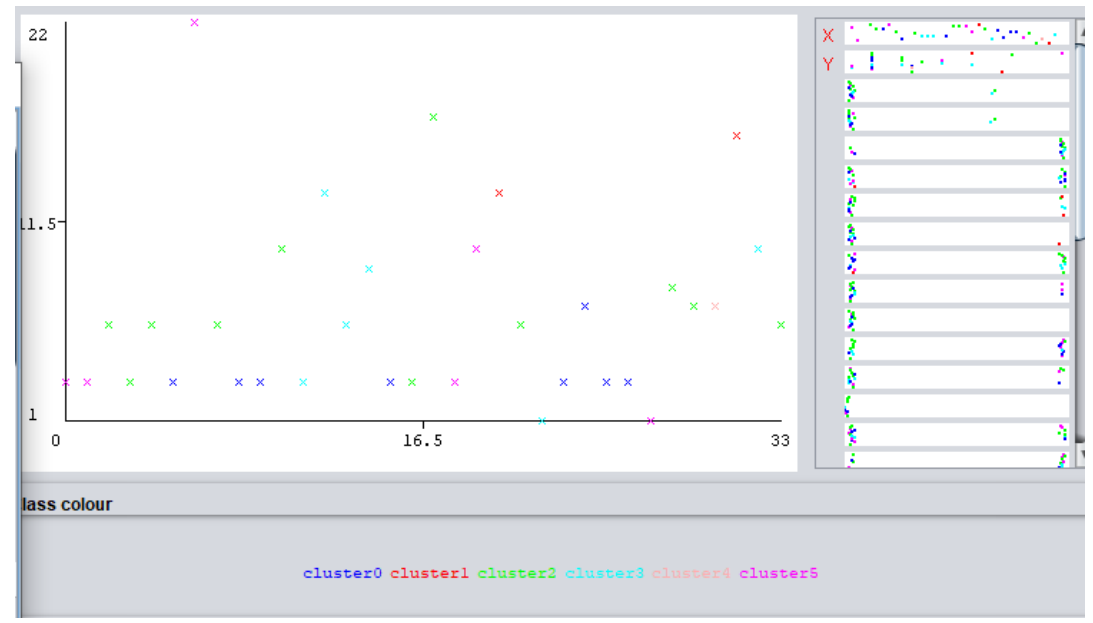

Figure 5.9. Visualising Cluster Assignments

Another visualisation seen in Figure 5.10 shows those patients with all clusters of a seizure type and all occurrences are showing True (red) or False (blue) for Sensory. In this example it shows the selected cluster (in red circle) whereby the seizure type is 'Multiple' with 'Sensory' for 'Key Signs \& Symptoms' and 'Multiple Weekly (up to 5)' as indicated on X axis.

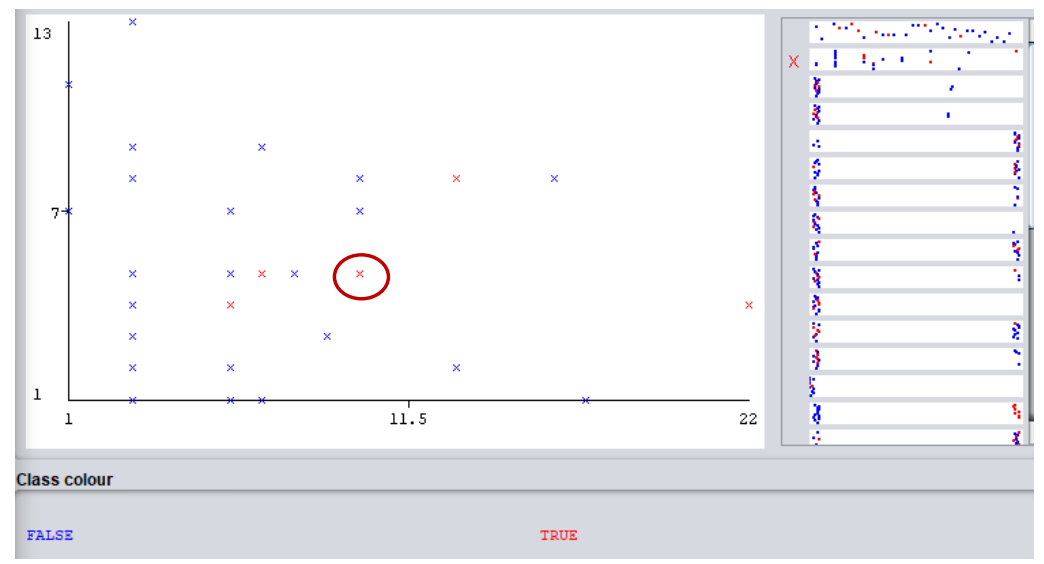

Figure 5.10. Seizure Type Clusters

\subsection{Summary}

In summary, this chapter has described the process followed to derive clusters groups of patients based on shared characteristics in their seizure symptoms and signs. The clustering algorithm used is the K-means algorithm in WEKA and the resulting 6 clusters show strong similarities in each cluster group using 'seed 10' and producing a mean sum of squared error of '188.07751'. As the clusters are now constructed, a list of typical seizure type patients can be produced and which cluster they belong to. Then a specific cluster can be taken, and the patient characteristics can be studied along these known dimensions. 


\section{Chapter 6 Experiment, Observations and Findings}

\subsection{Introduction}

This chapter discusses the experiments that are performed to capture seizure data, obtained from sensors, which are positioned on different parts of the patient's body. This is done to test the assumption that it is 'the individual profile' that makes the difference in which device to choose. This chapter corresponds to the methodology stage: 'I.V. PMP Framework \& Testing Stage' shown in Figure 3.1 Research Design Framework and addresses 'D1.Experimental Procedures'. The results from this experiment will be used to inform a typical model or a PMP (Personalised Monitoring Plan) discussed in the next chapter. This chapter will show a concise analysis of results from the test carried out.

\subsubsection{Experiment Procedures}

It is already becoming known that wearing sensors on the body is starting to be popular, as observed recently in a 2018 study where a great interest was highlighted in the use of wearable technology for epilepsy carers, this being independent of demographic and clinical factors and remarkably outpacing data security and technology usability concerns thus demonstrating the vital factor of comfortability [210]. Yet as discovered during a review to select the best sensor for each individual patient there was limited data on which was the best sensor for each seizure type, this was unfortunate despite an internationally active research effort, signifying the gap in knowledge, again, for understanding the individual epilepsy patient [211].

The actual 'sensor', and their 'position' (worn by the patient) are significant for epilepsy and the focus in this experiment is on how patients exhibit behaviour, rather than any actual testing of devices. It was therefore important to choose the most accurate sensors for monitoring epilepsy; these are found to be the accelerometer and heart-rate sensors, although latest studies suggest making use of other sensors too such as peripheral temperature, photo plethysmography (blood circulation), respiratory sensors [212], and galvanic (changes in sweat gland activity) among others [213].

Numerous studies have been previously been conducted with sensors and use for epilepsy [214] [215]. Since the 'gold standard' for epilepsy monitoring is video-EEG monitoring (which takes place within hospitals) [216] the driving questions addressed here were: 
$>$ Can the patient be just as accurately monitored at home with an inexpensive, easily obtainable accelerometer and heart-rate sensor-based device?

$>$ Can the individual requirements of the patient be pinpointed? If so, is it possible that these sensors can be worn at home (a personalised approach) and be just as effective as using EEG monitoring in the hospital setting?

From the analysis of the patient data it is clear that a patient profile based on particular characteristics can indicate which position the sensor is best placed on the patient's body.

Sample patient profiles where selected based upon criteria informed from discussions with clinicians. For example, Patient Profile 1 seizures begins with the right arm suddenly raising, therefore can the sensor be placed upon the right shoulder? Patient Profile 4 has a lot of shaking during their Focal Onset Seizures with shaking starting on the left arm so therefore can the sensor be useful attached to the left wrist? Whereas Patient Profile 5 begins their seizures with severe tremors on the right leg, can the sensors detect movement and heart-rate changes with sensor in this position? This analysis is further discussed in Chapter 7 in the 'Sensor Advisory'(section 7.4.1).

During the investigation practicable devices to use in the experiment to monitor epilepsy were analysed.

Table 6.1. Analysis of Practicable Devices for Experiment shows a list of possible devices, useful platforms or apps with their data export option and other sensors (that may be useful for future use) and notes (to assist decision).

In summary the 'Fitbit Ionic' is chosen as the best option since both the heart-rate and accelerometer can be extracted. The commercial activity device has been used in other studies, most notably recently whereby it used data from more than 47,000 Fitbit users in five U.S. states and data revealed that with Fitbit use the state-wide predictions of flu outbreaks were enhanced and accelerated [217]. This use demonstrates the viability and potential suitability of Fitbit as a healthcare device. 
Table 6.1. Analysis of Practicable Devices for Experiment

\begin{tabular}{|c|c|c|c|c|}
\hline Device/Platform/App & $\begin{array}{l}\text { Heart-Rate } \\
\text { Data Export } \\
\text { Option }\end{array}$ & $\begin{array}{l}\text { Accelerometer } \\
\text { Data Export } \\
\text { Option }\end{array}$ & $\begin{array}{l}\text { Other } \\
\text { Sensor }\end{array}$ & Notes \\
\hline \multicolumn{5}{|c|}{$[k e y] \sqrt{ }$ yes $X$ no } \\
\hline Mi Band & $X$ & $X$ & No & \\
\hline $\begin{array}{l}\text { Fitabase (data } \\
\text { management } \\
\text { platform) }\end{array}$ & $\sqrt{ }$ & $X$ & No & $\begin{array}{l}\text { Works with Fitbit device } \\
\text { only. Excellent solutions for } \\
\text { researchers to extract HR } \\
\text { data }\end{array}$ \\
\hline Acti-Graph & $\sqrt{ }$ & $\sqrt{ }$ & No & $\begin{array}{l}\text { Wear Location: wrist, } \\
\text { waist, ankle, thigh. } \\
\text { Important: these sensors } \\
\text { are not on one device. }\end{array}$ \\
\hline $\begin{array}{l}\text { Activ Insights (Geni- } \\
\text { Active }\end{array}$ & $X$ & $\sqrt{ }$ & No & \\
\hline \begin{tabular}{|l} 
Empatica E4 \\
Wristband: \\
Or Embrace Wrist \\
Watch
\end{tabular} & $\sqrt{ }$ & $\sqrt{ }$ & Yes & \\
\hline Fitbit Ionic & $\sqrt{ }$ & $\sqrt{ }$ & No & \\
\hline Fitbit Squash App & $\sqrt{ }$ & $X$ & No & $\begin{array}{l}\text { An easy to use App to use } \\
\text { with Fitbit }\end{array}$ \\
\hline $\begin{array}{l}\text { Medio Tek (mobile } \\
\text { health technology) }\end{array}$ & $\sqrt{ }$ & $X$ & $\begin{array}{l}\text { Oxygen } \\
\text { saturation, } \\
\text { respiratory } \\
\text { rate and } \\
\text { skin } \\
\text { temperature }\end{array}$ & \\
\hline Microsoft Band 2 & $\sqrt{ }$ & $\sqrt{ }$ & Skin & $\begin{array}{l}\text { Used with Free IoT Tools } \\
\text { App }\end{array}$ \\
\hline
\end{tabular}

\subsection{Experiment Description and Results}

Experiments were designed to gather data from sensors. The results of these tests will be used to inform the development of the PMP framework in Chapter 7.

This experiment is a, non-randomised, non-interventional study and is not intended as a device trial as it is based on existing technology and sensors. It is not intended to assess the device 
performance rather the sensor compatibility with the patient so that difference in behavior can be analysed.

Participants with confirmed epilepsy are recruited. The study targeted 10 epilepsy patients but only 6 were recruited. The data from the sensors will be collected after during a 5-day observation period.

The non-invasive wrist, leg, knee or arm-worn sensors are used to acquire heart-rate activity and movements.

\subsubsection{Objectives}

The objectives of this experiment are to assess the movement from the accelerometer sensor and the pulse from the heart-rate sensor in the detection of epileptic seizures

This study evaluates:

(1) The movement from the accelerometer sensor and the pulse from the heartrate sensor in the detection of an epileptic seizure. Over a period of 5 days they will be asked to wear the device and continue recording seizures in their seizure diary.

(2) Any differences in result due to the 'position' of the sensor on the body together with the patients' acceptability \& comfort

Secondary objectives

(3) To investigate whether this study can pin-point a patient's individual requirements to improve their daily management of epilepsy

(4) To investigate if the sensors worn are just as effective as detecting seizure data in the patient's seizure diary

\subsubsection{Eligibility Criteria}

The patients' medical records are used to determine their eligibility for the study.

Additionally, pre-determining eligibility reduces the burden on patient's time and help ensure only those who meet the strict inclusion criteria for the study are included. The information obtained, other than participant grouping, from the participants medical records is not shared within the research team. 


\section{Inclusion criteria for patients:}

(i) Patients who have a confirmed diagnosis that epileptic seizures occur

(ii) Patients who are having frequent episodes of epileptic seizures; more than 2-3 per week.

(iii) Patients that are willing to record seizures in a seizure diary.

(iv) Patients that are confident using technology.

(v) Between the ages of $18-80$

\section{Exclusion criteria for patients:}

(i) Patients that have non-epileptic seizures.

(ii) Patients that have less frequent seizures; so less than 2 per week

(iii) Patients that are not confident using technology.

(iv) Patients that are unable or not willing to record seizures in a seizure diary

(v) Inability to comply with the trial procedure, such as cognitive or behavioural problems

(vi) Inability to give informed consent

\subsubsection{Wearable Sensors}

This experiment intends to monitor bio-signals using the non-invasive wrist, leg, knee or armworn Fitbit Ionic containing sensors. Two sensors are used during the study: accelerometer and heart-rate sensor. There is one Fitbit Ionic used containing two sensors: accelerometer and heartrate monitor. These sensors are described below:

- Accelerometer Sensor: sensor captured motion-based activity and can be used for measuring the acceleration of a moving or vibrating body

- Heart-Rate Sensor: uses green LED lights paired with light-sensitive photodiodes to detect the amount of blood flowing through the wrist (or other body part) at any given moment. When the heart beats, the blood flow in the wrist and the green light absorption is greater [212]. 
The patient is also issued with instructions. These instructions explain how to begin the monitoring using the device and also how to wear it in the recommended position, see example below: Figure 6.1 and Figure 6.2.

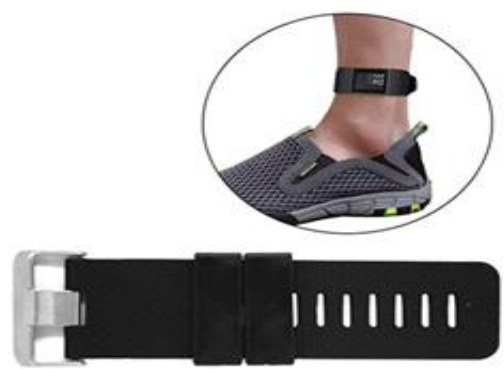

Figure 6.1. Position on ankle

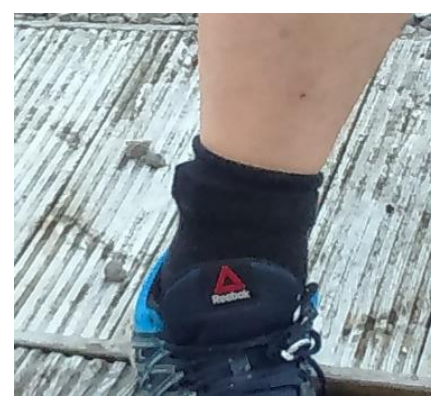

Figure 6.2. Inside

Sock

Participants are asked to wear the Fitbit Ionic with the sensors for 1 week. They are asked to remove it for charging and for bathing. The device will require charging once every 5 days, the participants receive information and accessories to charge their sensor-based device.

The instructions contain daily forms for the patient to complete, hence, keeping a diary of the times of seizure, if they do not use this method an EEG recording will be obtained from hospital staff. This way the actual time stamp of the patients recorded seizure can be checked against the server time stamp observations of the seizure, so for example if the patient records their seizure at $10.20 \mathrm{am}$ and the server readings reveal heart-rate peaks and rapid movement from the accelerometer also at 10.20am, then this confirms the server readings match the patients (or EEG) known seizure occurrence (see Figure 6.3. Seizure Time Stamps).

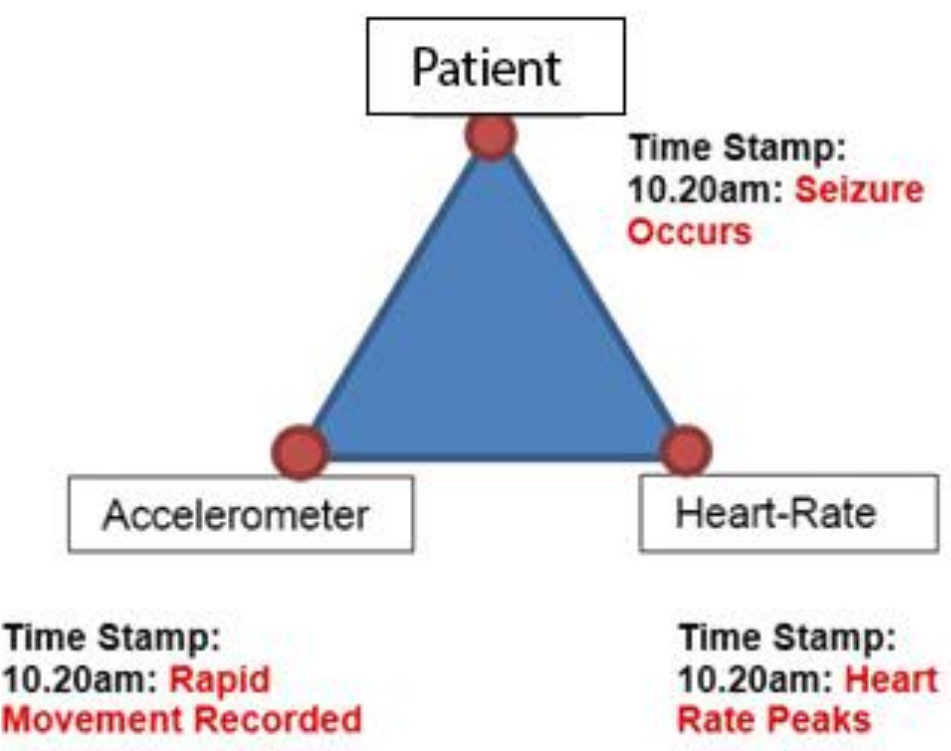

Figure 6.3. Seizure Time Stamps 


\subsubsection{Data Collection \& Analysis}

The artefacts collected at this stage are:

- Number of seizures recorded in patient's seizure diary or from EEG

- Data collected from the wearable sensors. This data will be synced daily and uploaded to the server.

The captured epileptic seizures will be measured. This will be measured against the patient's seizure diary or EEG confirmed recordings from the hospital. The data produced from the sensors for each individual patient will be time-stamped with the seizure diary (or EEG) and data analysis will determine what was happening before, during and after a seizure occurs. The back-end monitoring process can be seen in APPENDIX A 'Backend Monitoring'.

\subsubsection{Pilot Study}

A pilot study took place to calibrate the experiment design. Preliminary experiments with the Fitbit Ionic device were performed, and simulated data from the accelerometer and heart-rate monitor sensors were collected.

There were certain aspects to consider and modify due to the experience during the pilot study such as identified in Table 6.2. Pilot Study Aspects \& Modifications.

Table 6.2. Pilot Study Aspects \& Modifications

\begin{tabular}{|l|l|}
\hline Aspects & Modifications \\
\hline $\begin{array}{l}\text { The battery power ceased after 5 } \\
\text { days. }\end{array}$ & $\begin{array}{l}\text { The participants need to } \\
\text { receive clear instructions } \\
\text { on how to charge the } \\
\text { device. }\end{array}$ \\
$\begin{array}{l}\text { result, although data captured up to } \\
\text { that point is secure. }\end{array}$ & $\begin{array}{l}\text { To test this, a "force-run- } \\
\text { down" on the battery was } \\
\text { performed by running an } \\
\text { There was no indicator that once } \\
\text { immediately starts capturing data } \\
\text { and the APP is re-started. }\end{array}$ \\
$\begin{array}{l}\text { infice } \\
\text { device. }\end{array}$ \\
\hline
\end{tabular}




\begin{tabular}{|c|c|}
\hline & $\begin{array}{l}\text { The assurance is that the } \\
\text { device begins capturing } \\
\text { immediately. }\end{array}$ \\
\hline $\begin{array}{l}\text { Slow reading of data prompted } \\
\text { concerns about the limited memory } \\
\text { space in the device and data } \\
\text { handling to store further data. }\end{array}$ & $\begin{array}{l}\text { It was discovered in the } \\
\text { original specifications that } \\
\text { Ionic has } 2.5 \text { G.B storage } \\
\text { space. Yet during data } \\
\text { collection storage exceeded } \\
\text { this limit and did not pose a } \\
\text { problem. } \\
\text { Therefore, is was possible } \\
\text { to record and measure data } \\
\text { every } 30 \text { seconds rather } \\
\text { than every minute. }\end{array}$ \\
\hline $\begin{array}{l}\text { It was found that file size } \\
\text { restrictions existed within the } \\
\text { device. }\end{array}$ & $\begin{array}{l}\text { New files after a file size } \\
\text { threshold were created to } \\
\text { deal with this. }\end{array}$ \\
\hline
\end{tabular}

\subsection{Experiment Results}

Six hospital patients were enrolled, but one patient excluded due to technical difficulty obtaining both the heartrate and accelerometer data. Two volunteers where enrolled and performed seizures in a simulated environment. Table 6.3 provides known patient profile characteristics and reported seizure types.

Hospital patients wore the sensor-based device for 5 days during video and EEG monitoring within hospital settings, all observations are in APPENDIX B 'Hospital Patient Observations'. A total of 24 seizures were recorded with video and EEG: 9 GTCS, 14 non-GTCS and 1 noneepileptic event. Two volunteer patients simulated total of 8 seizures. In total 32 seizure events were captured by the sensor-based device. 2 events recorded by video and EEG were missed (for HP4) as the patient was either not physically wearing the watch at the time of a GTCS or the data was skipped due to technical difficulty. The sensor-based device consequently detected seizures effectively which were recorded in seven participants. 


\begin{tabular}{|c|c|c|c|c|c|c|c|c|}
\hline $\begin{array}{l}\text { Patient } \\
\text { Profile }\end{array}$ & $\begin{array}{l}\text { Amount } \\
\text { of } \\
\text { Seizures }\end{array}$ & Seizure Type & $\begin{array}{l}\text { Dominant } \\
\text { Side }\end{array}$ & $\begin{array}{l}\text { Key Signs \& } \\
\text { Symptoms }\end{array}$ & $\begin{array}{l}\text { Common Signs \& } \\
\text { Symptoms }\end{array}$ & Time of Day & Notes \& Considerations & $\begin{array}{l}\text { Clustering } \\
\text { Group }\end{array}$ \\
\hline $\begin{array}{l}\text { Excluded } \\
\text { Patient }\end{array}$ & 2 & $\begin{array}{l}\text { GTCS (Generalised } \\
\text { Tonic Clonic Seizure) }\end{array}$ & Right & Clonus & $\begin{array}{l}\text { Sensory } \\
\text { Cognitive }\end{array}$ & Diurnal & $\begin{array}{l}\text { Heart-rate data collected } \\
\text { but accelerometer did not } \\
\text { work. } \\
\text { The patient used the } \\
\text { paper-based diary to } \\
\text { record seizures. }\end{array}$ & Cluster Group 4 \\
\hline $\begin{array}{l}\text { Patient } 1 \\
\text { (HP1) }\end{array}$ & 14 & $\begin{array}{l}\text { FAS (Focal Aware } \\
\text { Seizure) also known } \\
\text { as CPS } \\
\text { FSIA (Focal seizures } \\
\text { with impaired or loss } \\
\text { of awareness.) }\end{array}$ & $\begin{array}{l}\text { Seizures } \\
\text { begin on the } \\
\text { Right. }\end{array}$ & LOC & Automatisms & Diurnal & $\begin{array}{l}\text { Continues to have seizures } \\
\text { despite surgery. } \\
\text { Seizures usually lasting } \\
10-20 \text { seconds. }\end{array}$ & Cluster Group 2 \\
\hline $\begin{array}{l}\text { Patient } 2 \\
\text { (HP2) }\end{array}$ & $\begin{array}{l}\text { No } \\
\text { seizures } \\
\text { occurred }\end{array}$ & $\begin{array}{l}\text { FSIA (Focal seizures } \\
\text { with impaired or loss } \\
\text { of awareness.) }\end{array}$ & $\begin{array}{l}\text { Both Left } \\
\text { and Right }\end{array}$ & $\begin{array}{l}\text { LOC } \\
\text { Clonus }\end{array}$ & Automatisms & Diurnal & $\begin{array}{l}\text { Also, but (rare) secondary } \\
\text { GTCS. R temporal } \\
\text { cavernoma (small vascular } \\
\text { malformation) but } \\
\text { surprisingly the EEG }\end{array}$ & Cluster Group 2 \\
\hline
\end{tabular}




\begin{tabular}{|c|c|c|c|c|c|c|c|c|}
\hline & & $\begin{array}{l}\text { GTCS (Generalised } \\
\text { Tonic Clonic Seizure) }\end{array}$ & & & & & $\begin{array}{l}\text { changes during seizures } \\
\text { (ictal onset zone) was over } \\
\text { the Left temporal lobe. }\end{array}$ & \\
\hline $\begin{array}{l}\text { Patient } 3 \\
\text { (HP3) }\end{array}$ & $\begin{array}{l}\text { No } \\
\text { seizures } \\
\text { occurred }\end{array}$ & $\begin{array}{l}\text { GTCS (Generalised } \\
\text { Tonic Clonic Seizure) } \\
\text { FSIA (Focal seizures } \\
\text { with impaired or loss } \\
\text { of awareness.) }\end{array}$ & $\begin{array}{l}\text { Both Left } \\
\text { and Right }\end{array}$ & $\begin{array}{l}\text { LOC } \\
\text { Clonus }\end{array}$ & Automatisms & $\begin{array}{l}\text { Nocturnal } \\
\text { (GTCS) } \\
\text { Diurnal } \\
\text { (Focal } \\
\text { Seizures) }\end{array}$ & $\begin{array}{l}\text { No seizures captured } \\
\text { despite patient reporting } \\
\text { frequent seizures at home. }\end{array}$ & Cluster Group 4 \\
\hline $\begin{array}{l}\text { Patient } 4 \\
\text { (HP4) }\end{array}$ & 9 & $\begin{array}{l}\text { GTCS (Generalised } \\
\text { Tonic Clonic Seizure) } \\
\text { FSIA (Focal seizures } \\
\text { with impaired or loss } \\
\text { of awareness.) }\end{array}$ & $\begin{array}{l}\text { Seizures } \\
\text { begin on the } \\
\text { Right. }\end{array}$ & $\begin{array}{l}\text { LOC } \\
\text { Clonus }\end{array}$ & $\begin{array}{l}\text { Automatisms } \\
\text { Emotions } \\
\text { (behavioural } \\
\text { change) }\end{array}$ & Nocturnal & $\begin{array}{l}\text { Video EEG captured } \\
\text { secondary generalised. All } \\
\text { seizures from sleep. }\end{array}$ & Cluster Group 4 \\
\hline $\begin{array}{l}\text { Patient } 5 \\
\text { (HP5) }\end{array}$ & 1 & $\begin{array}{l}\text { Non-epileptic } \\
\text { (Unclassified) }\end{array}$ & Left & Bilateral Clonus & Cognitive & Diurnal & $\begin{array}{l}\text { No epileptic seizures but } \\
\text { one non epileptic shaking } \\
\text { event recorded. }\end{array}$ & Cluster Group 1 \\
\hline
\end{tabular}




\begin{tabular}{|c|c|c|c|c|c|c|c|c|}
\hline \multicolumn{9}{|c|}{ Simulation } \\
\hline $\begin{array}{l}\text { Patient } \\
\text { Profile }\end{array}$ & $\begin{array}{l}\text { Amount } \\
\text { of } \\
\text { Seizures }\end{array}$ & Seizure Type & $\begin{array}{l}\text { Dominant } \\
\text { Side }\end{array}$ & $\begin{array}{l}\text { Key Signs \& } \\
\text { Symptoms }\end{array}$ & $\begin{array}{l}\text { Common Signs \& } \\
\text { Symptoms }\end{array}$ & Time of Day & Notes \& Considerations & $\begin{array}{l}\text { Clustering } \\
\text { Group }\end{array}$ \\
\hline $\begin{array}{l}\text { Volunteer } \\
1 \\
\text { (V1) }\end{array}$ & 4 & $\begin{array}{l}\text { GTCS (Generalised } \\
\text { Tonic Clonic Seizure) }\end{array}$ & Right & Bilateral Clonus & & $\begin{array}{l}\text { Diurnal \& } \\
\text { Nocturnal }\end{array}$ & & Cluster Group 4 \\
\hline $\begin{array}{l}\text { Volunteer } \\
2 \\
\text { (V2) }\end{array}$ & 4 & GTCS & Left & Bilateral Clonus & & Diurnal & & Cluster Group 4 \\
\hline
\end{tabular}

Table 6.3. Known Patient Characteristics 
All seizure events detected by the sensor-based device recorded the correct date and time of the seizure, matching the time-stamp of the EEG video recording and not the 'patient diary'. Six out of 32 seizures occurred during sleep while, 26/32 seizures occurred during the day or night-time while the participants were awake. The durations of the events detected by the sensor-based device were visually captured in charts showing periods of 2-4 minutes (with the exact time of the event indicated): this time-scale varied from 2-4 minutes for each participant due to available data queried at the time from the server. The average duration for the seizure captured by the sensor-based device was 1 minute. Seizure event data was uploaded to the server and to the online database in $32 / 34(94.18 \%)$ of the seizures.

Non-seizure durations were also charted for each participant, in some cases false positives events occurred this due to any non-epileptic activity from tossing and turning in bed, cleaning teeth, stirring a drink or general daily activity.

None of the seizure events, except for the excluded patient, were recorded on the paper diary provided during the study.

\subsubsection{Findings and Discussion}

The heart-rate and accelerometer sensors used to detect characteristics of seizure events can successfully record seizure data, without need for participant cooperation beyond wearing the sensor-based device, even recharging the battery (battery life is 5 days when fully charged) was not required by the participants. Both the sensors detected the 'shaking' seizures correctly as seen from 2 observations below from HP4 in 'Figure 6.4' and 'Figure 6.5'.

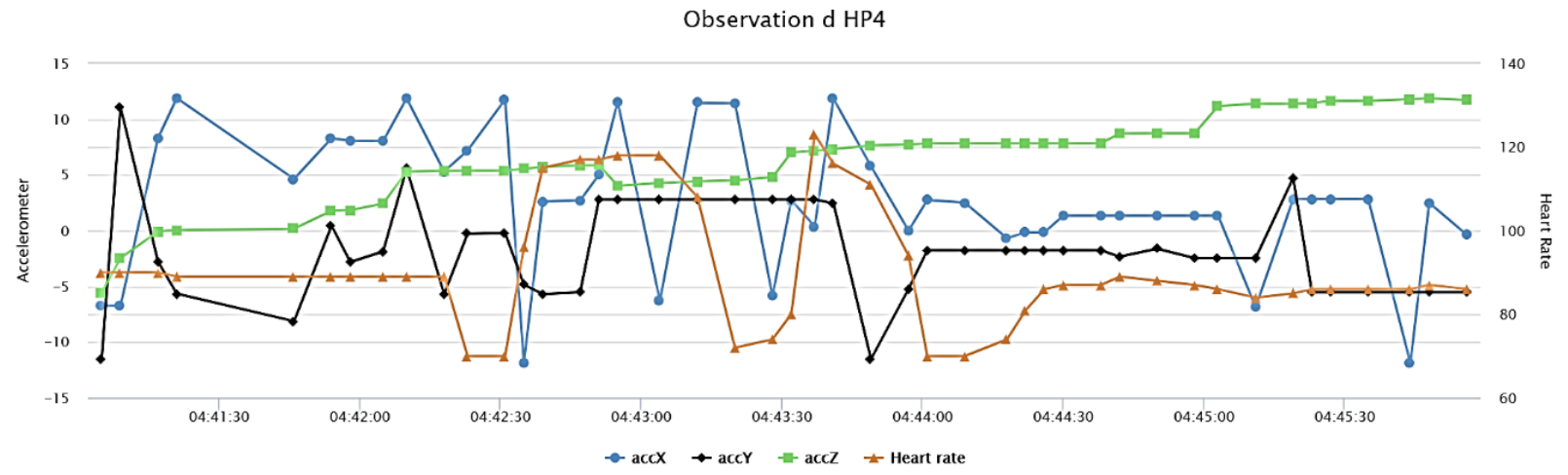

Figure 6.4. Observation d HP4 


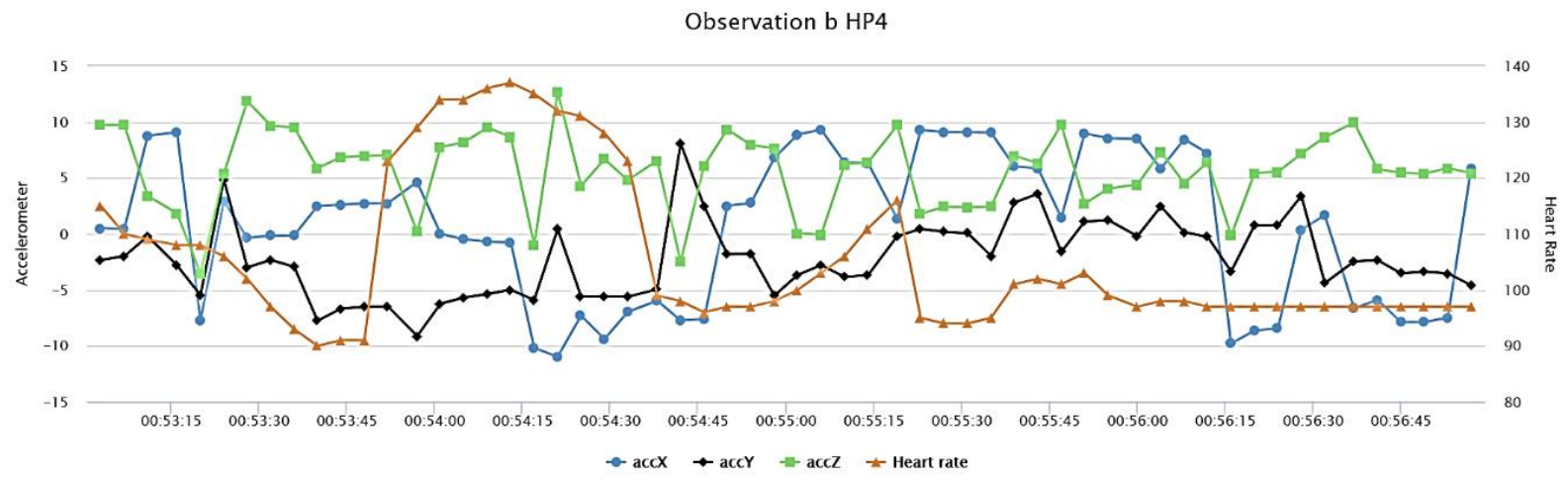

Figure 6.5. Observation b HP4

The effectiveness was also verified by "Non-seizure times" which are more easily recognised in sleep due to inactivity demonstrating that the sensors worked properly: see below random time periods whereby seizures did not occur for HP4 in Figure 6.6 and Figure 6.7.

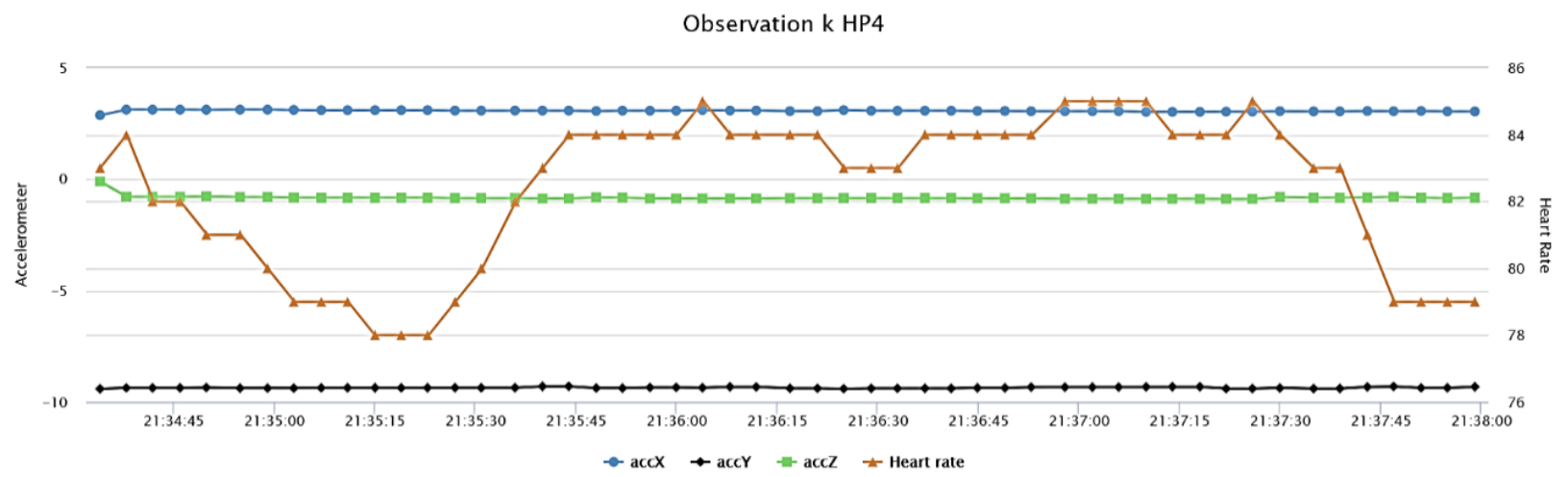

Figure 6.6. Observation k HP4

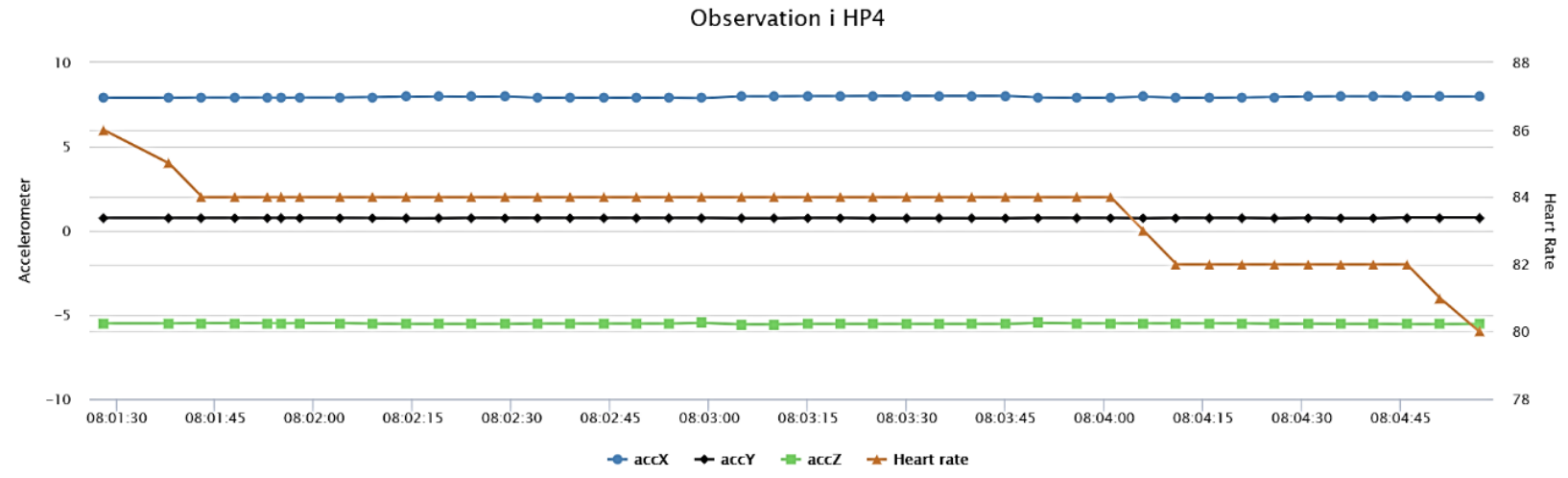

Figure 6.7. Observation i HP4

Both accelerometer and heart-rate sensors have been used to detect seizures in numerous previous studies [212] but in this study it was found that when used together in one device they did not always work in sync "together". This is because when the sensors are worn on the non-dominant side and seizure occurs only heart-rate change indicated: accelerometer shows no change. Yet when in correct position on the body they work in union as an excellent detection method. 
Therefore, demonstrating that body placement or position is paramount. For example, HP4s' dominant side is the right arm. This means seizures are known to occur on the right. The results from "Observation c HP4" (Figure 6.8), can be seen below. During shaking from the right wrist at the recorded time: 00.23am during a GTCS seizure all 3 measurements on axis $\mathrm{X}, \mathrm{Y}$ and Z showed sudden movements and the heart-rate increased to its highest peak at 128 . Before the seizure the heart-rate was much lower at 80 , then rising rapidly to 90 and up to 128 . This suggests both the sensors detected the seizure correctly.

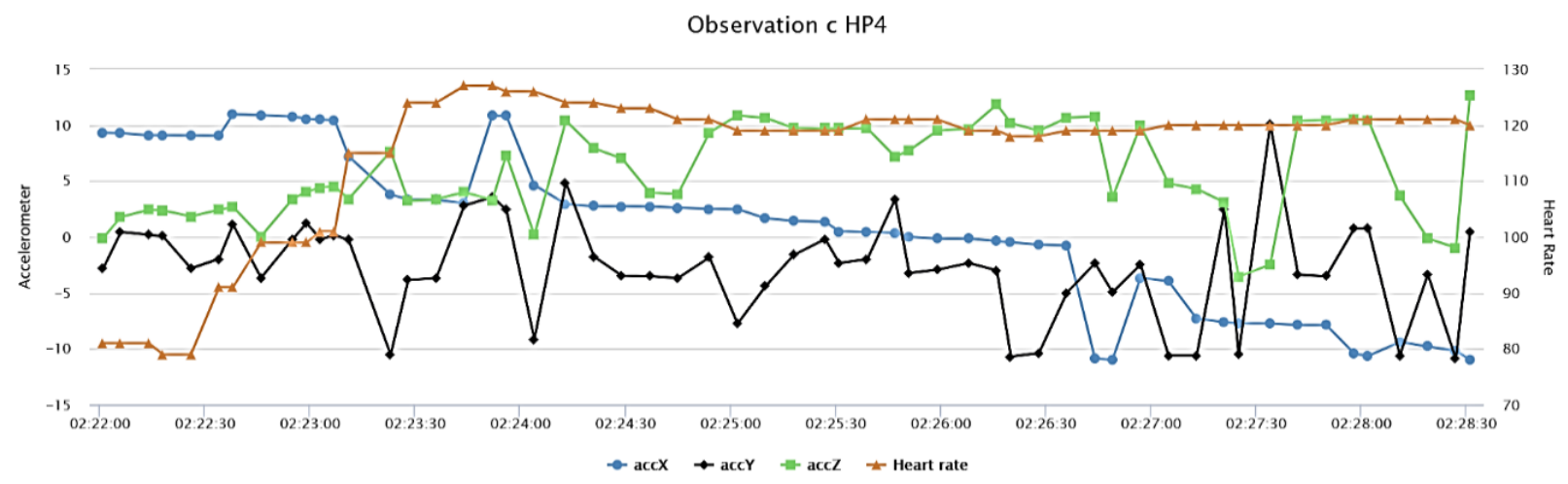

Figure 6.8. Observation c HP4

Yet the results from "Observation a HP4", seen below (Figure 6.9) indicate that during a GTCS at the recorded time ' $12.44 \mathrm{pm}$ ' the 3 measurements on axis $\mathrm{X}, \mathrm{Y}$ and $\mathrm{Z}$ did not show any sudden movement, in fact barely any movement at all, yet the heart-rate increased to its highest peak at 124, in keeping with typical heart-rate increase measurement during a GTCS for HP4. Since the accelerometer was positioned on the left wrist this reveals the sensor did not detect movement therefore demonstrating the sensor was positioned in the wrong position.

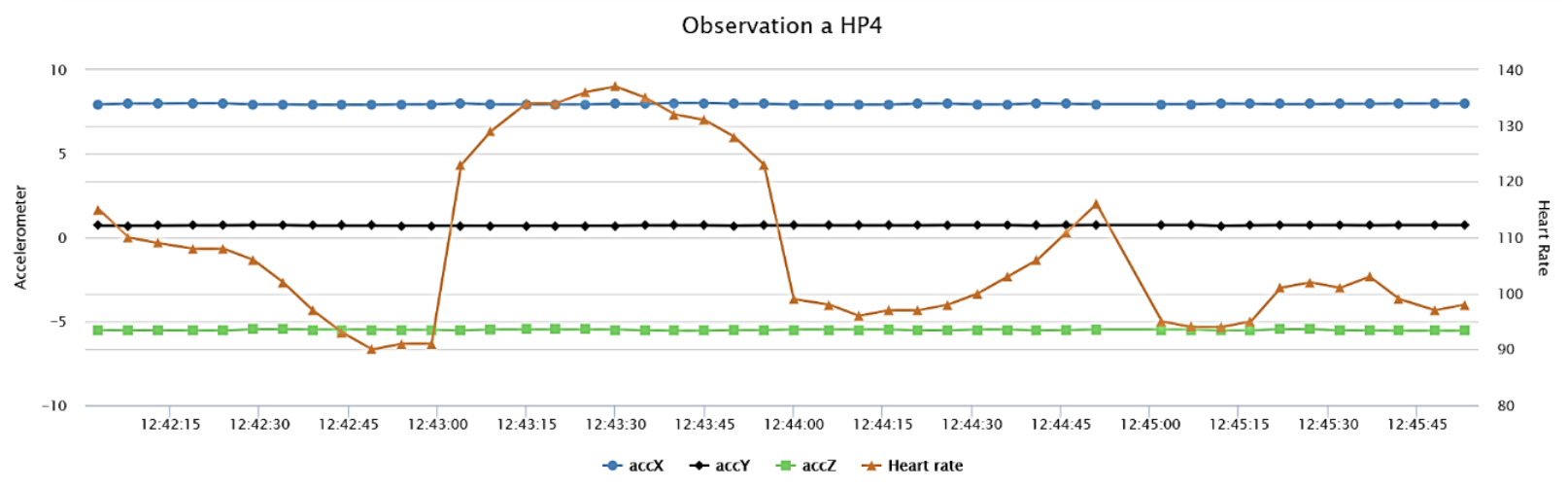

Figure 6.9. Observation a HP4

Knowing the individual characteristics of the patient profile prior to sensor-based device recommendation is key, for example the HP1 with FAS (Focal Aware Seizures) and FSIA (Focal Seizures with Impaired Awareness): the question here was "did the 2 sensors work in union to detect the Focal seizures?" Some heart-rate increase was detected but the accelerometer was 
primarily redundant, for example in 'Observation 10' (Figure 6.10) a seizure occurs with sensor positioned on right wrist at the observed time: 09.44am, in this observation the heart-rate sensor detects some change over a 2 minute period i.e. The heart-rate begins at 87 increases to 90 then back to 87 then declines to 86 then steadily back to 90 . At 09.45 am the heart-rate does show increase to 95 and goes back down to 85 . Heart-rate range is $87-95$, with some sudden movement from accelerometer at time of increased heart-rate.

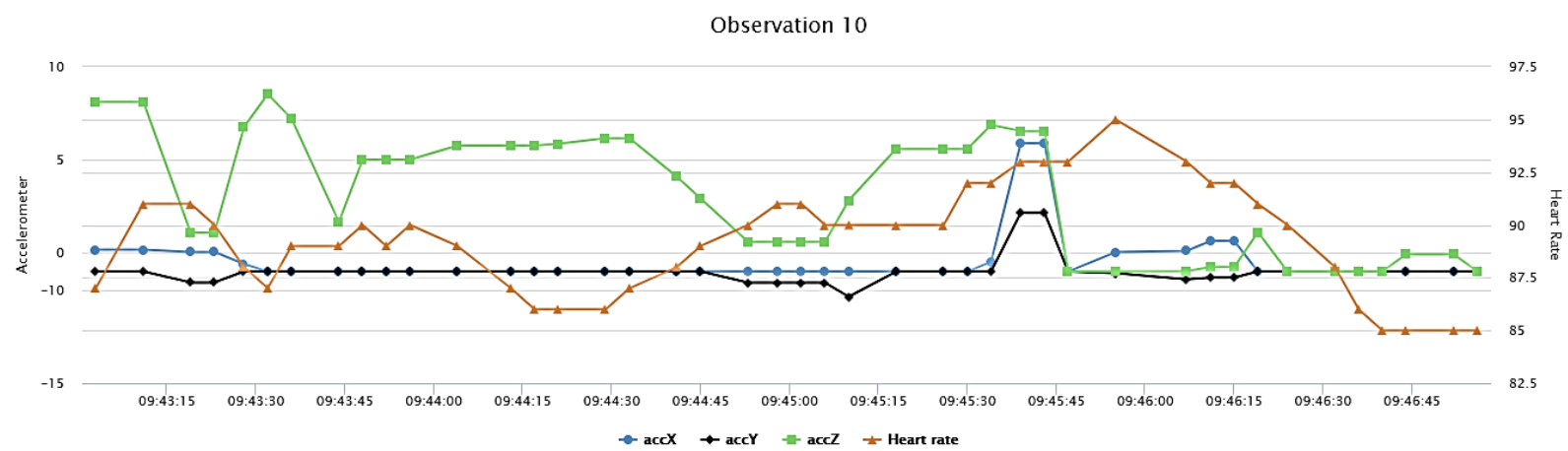

Figure 6.10. Observation 10

Yet, during 'Observation 11', seen below in Figure 6.11 for HP1 with the seizure observed at $20.36 \mathrm{pm}$ the heart-rate range is $81-84$ with little sudden movement. Likewise, in 'Observation $12^{\prime}$ (Figure 6.12) : the seizure occurrence at 21.06pm demonstrates the heart-rate range: $81-86$ and little sudden movement.

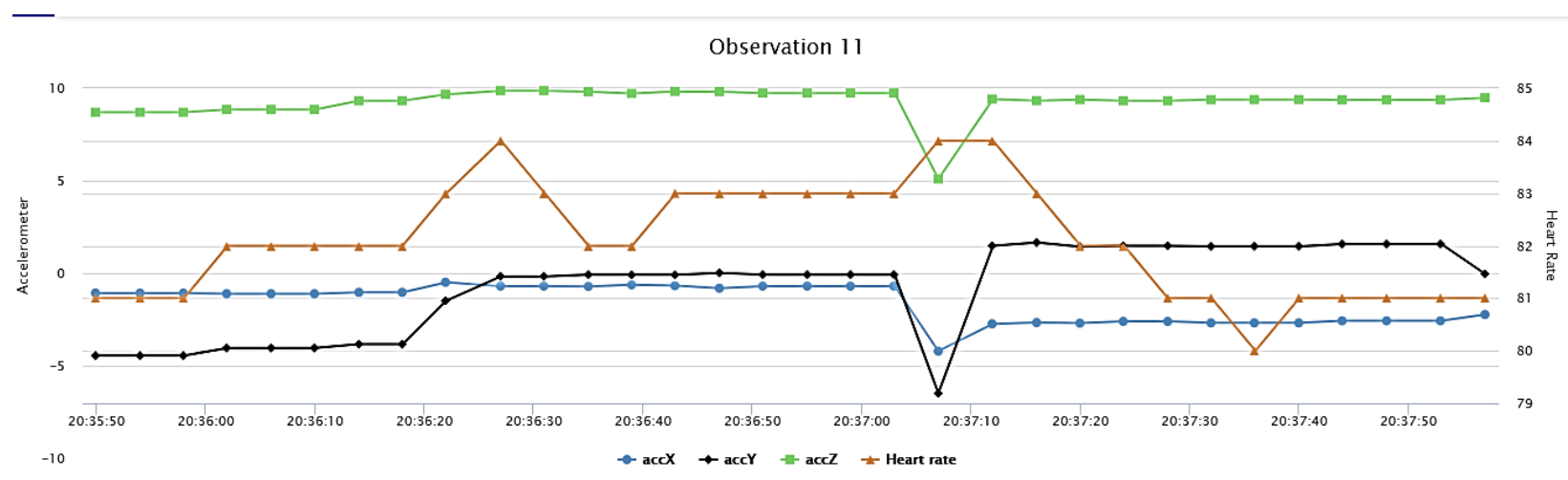

Figure 6.11. Observation 11 


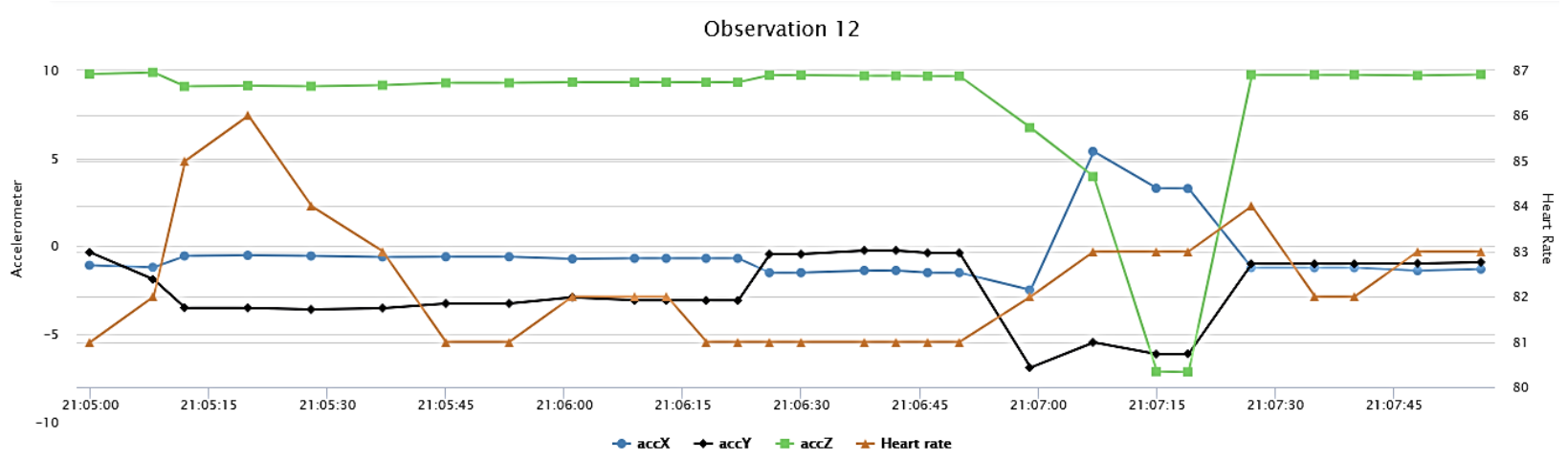

Figure 6.12. Observation 12

A pattern emerges for HP1 in other observations whereby the heart-rate decreases, for example in 'Observation a HP1' (in Figure 6.13 below) at the time of the seizure '09.55am' the heart-rate decreases from 90 to 84, and likewise in 'Observation f HP1' (Figure 6.14) with heart-rate decrease from 104 to 79 during the observed time of seizure at 11.01am and in 'Observation $\mathrm{g}$ HP1' (Figure 6.15) with heart-rate decrease from 100 to 84 during the observed time of seizure at $20.44 \mathrm{pm}$.

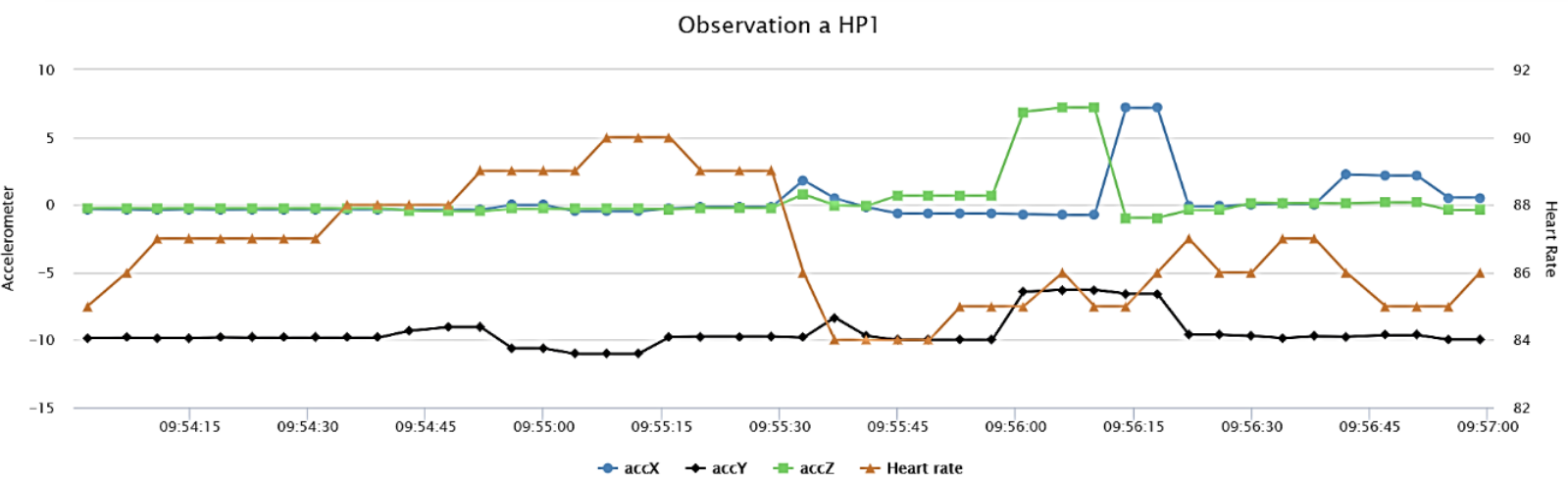

Figure 6.13. Observation a HP1

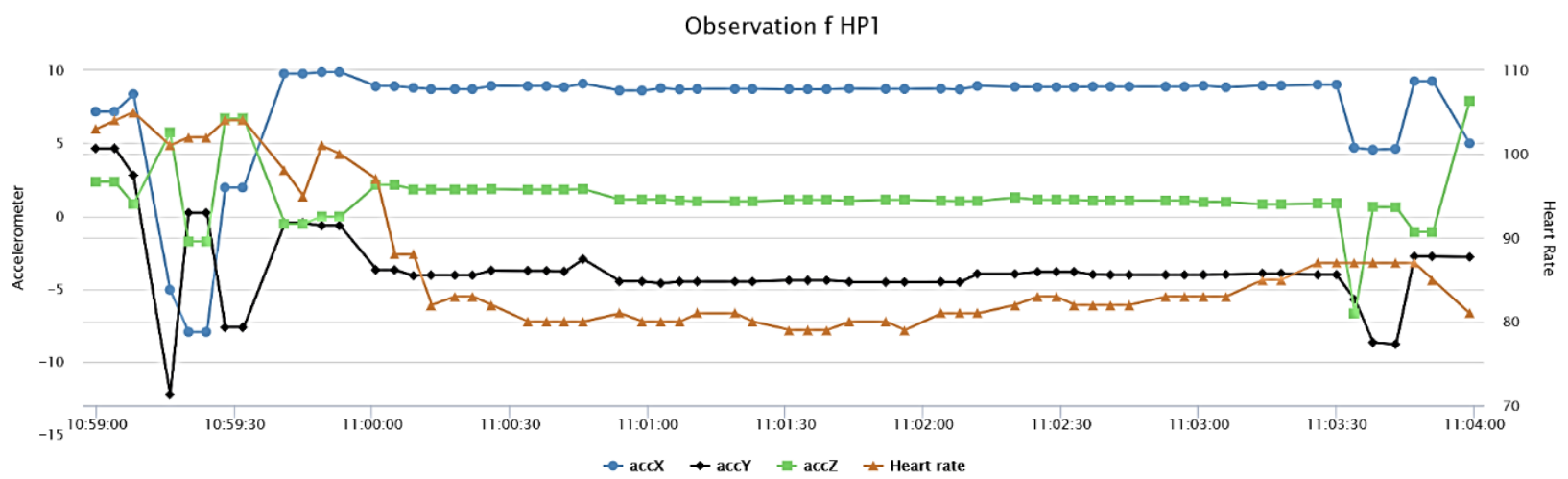

Figure 6.14. Observation f HP1 


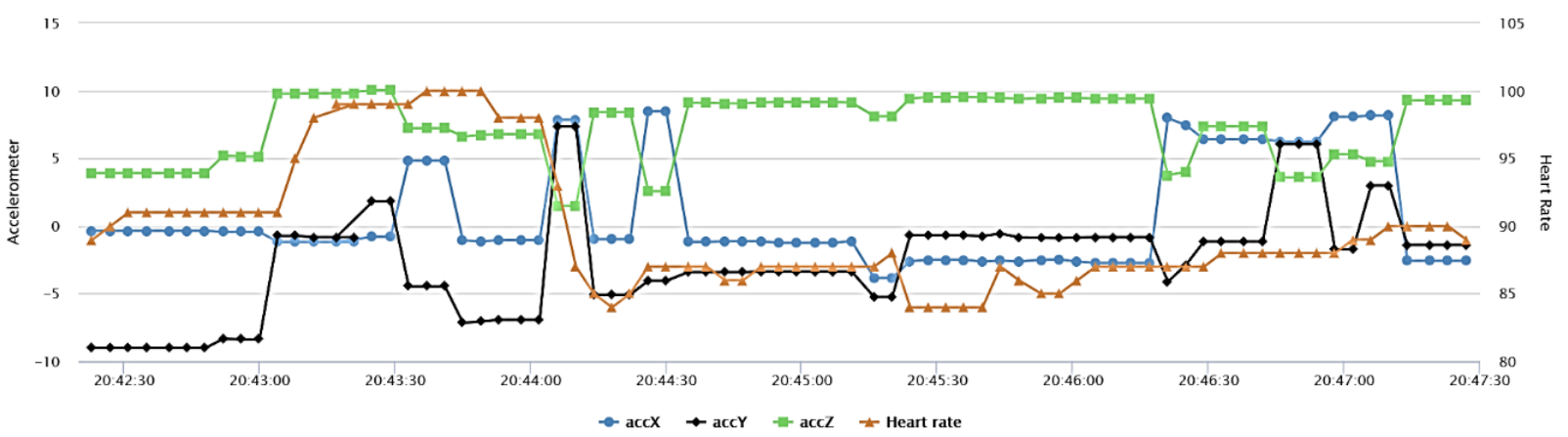

Figure 6.15. Observation g HP1

In 'Observation $\mathrm{g} \mathrm{HP1}$ ' the accelerometer indicates movement from all $3 \mathrm{X}, \mathrm{Y}$ and $\mathrm{Z}$ axis on the accelerometer at the time of the seizure. This is further observed in 'Observation e HP1' (in Figure 6.16) whereby there is sudden change in the accelerometer, but this is ' $21.01 \mathrm{pm}$ ' 'after' the time of seizure at ' 21.00 ' where in fact again the heart-rate shows decrease.

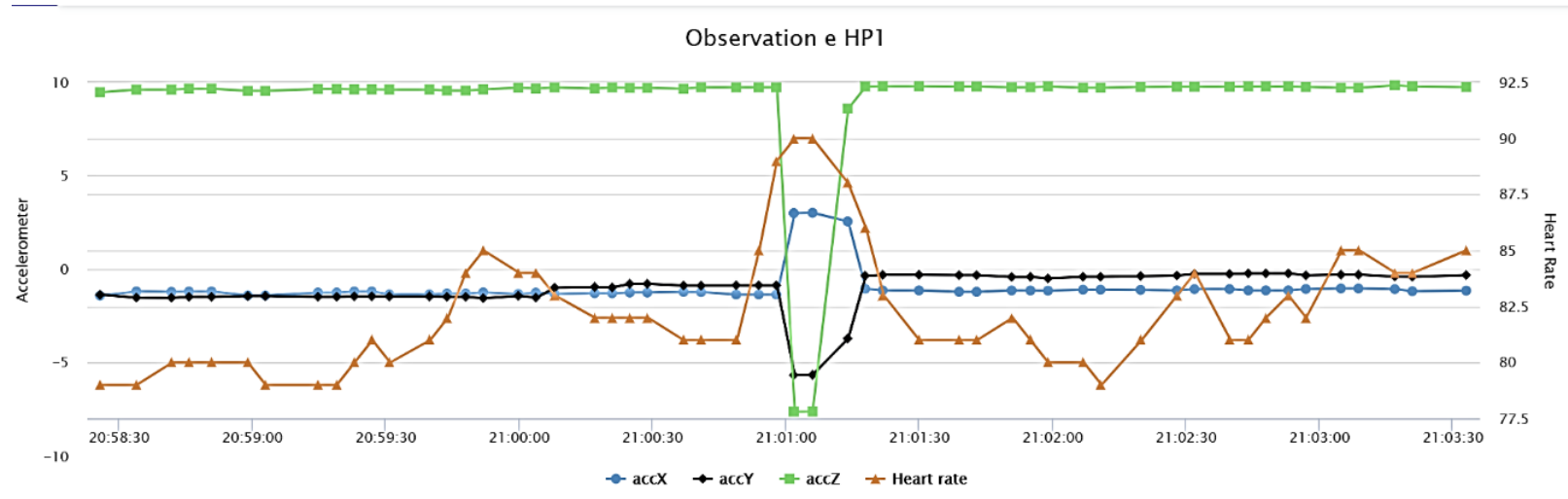

Figure 6.16. Observation e HP1

Only 1/14 seizures observed for HP1 demonstrate significant movement for the accelerometer during seizures. Therefore, the accelerometer sensor is not useful for detecting these non-shaking seizures, although some patients' profiles (found earlier in the collection of the 'anonymous patient profiles') reveal absence seizures can evolve to convulsive generalized tonic clonic movements. Therefore, it is endorsed that Focal seizures alone, go undetected without HR changes [3].

Predominantly during non-seizure time periods for HP1 there is no significant variance in accelerometer measurements when compared to 'seizure occurrence' time periods. For example, "Observation 16" (in Figure 6.17) shows a 4-minute snap-shot when HP1 has no seizure: the 3 axis X, Y and Z move similarly in "Observation 12" (in Figure 6.18). This is further evidenced in APPENDIX B where more non-seizure periods are demonstrated. 


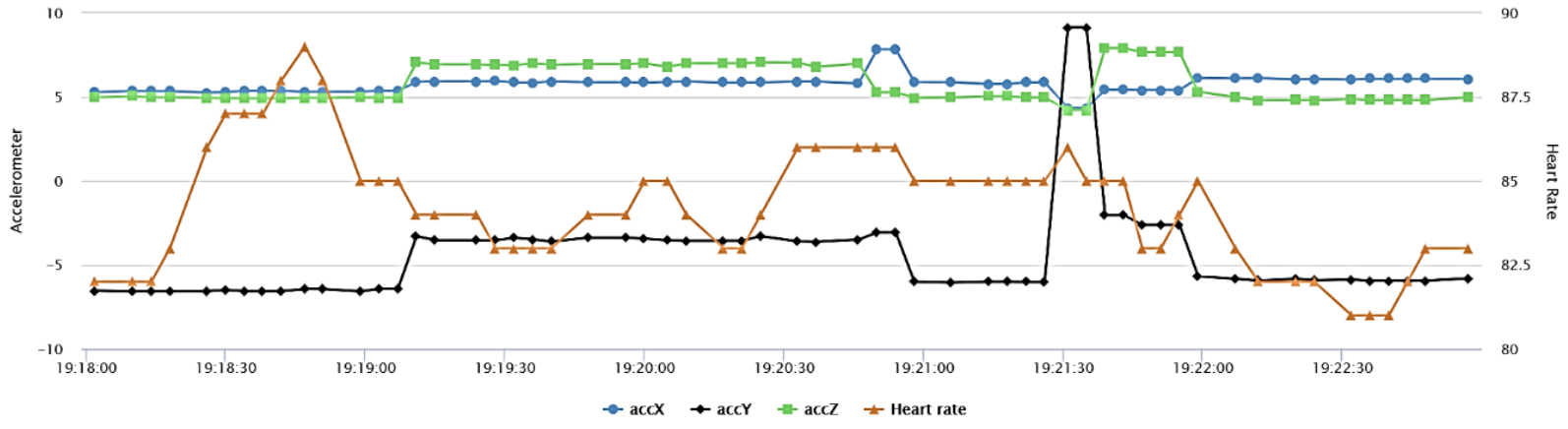

Figure 6.17. Observation 16

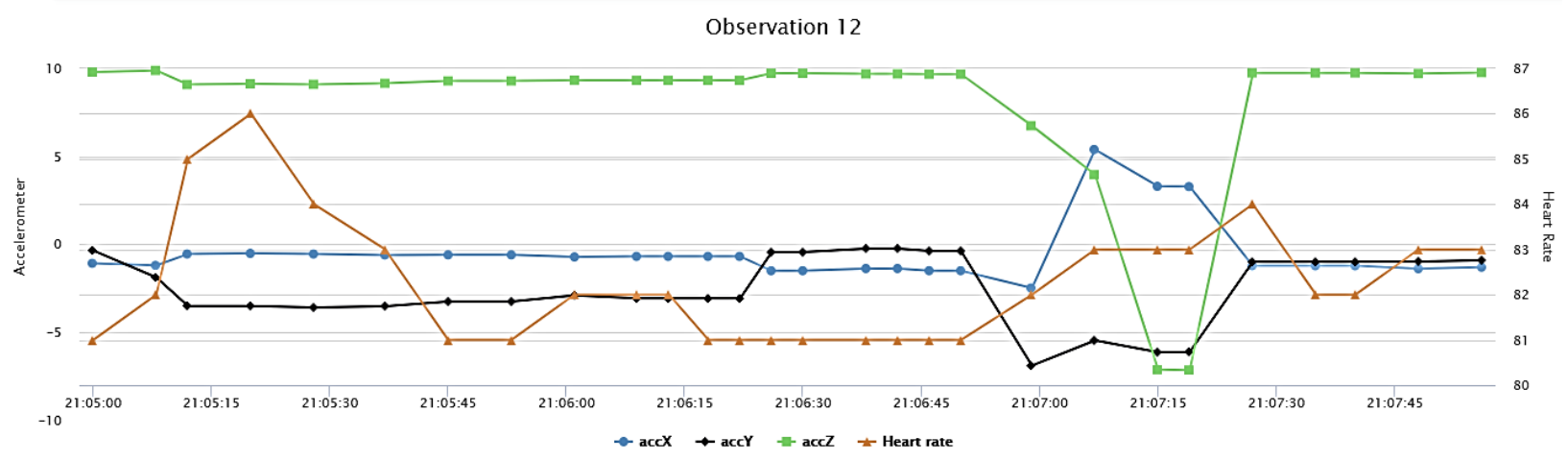

Figure 6.18. Observation 12

Ultimately there is some evidence demonstrated here that heart-rate 'change' occurs during the seizures for HP1 (increase and decrease). Yet since the seizures for HP1 occur for such a short time (20 seconds) heart-rate fluctuations can be missed or miss-interpreted as 'false alarms' perhaps due to agitation before or after the seizure.

The key and common signs and symptoms are 'LOC' (Loss of Consciousness) and 'Automatisms' for a patient such as HP1. Automatisms reveal themselves in a multitude of forms including repetitive movements, such as, lip smacking, chewing or swallowing, picking at clothes or skin or even staring [218] and these are difficult to detect with any sensor.

Hence, these repetitive movements deemed as other behavioural components of seizures which include non-motor components and post-ictal phenomena cannot be detected by the accelerometer [219] in line with the theory that seizures that are typical to the dominant body area not wearing the sensor-based device will not be detected.

Some patients exhibit automatisms such as sudden sweating events [220], and since sweating is associated with Focal Seizures a more appropriate sensor for a patient with FAS and FSIA seizure types would be Galvanic Skin Response Sensor (GSR), which refers to changes in sweat gland 
activity [57] as evidenced in other studies performed to detect seizures this galvanic skin response (GSR) sensor has been used in multi-modal platforms [220].

Empaticas' 'Embrace Plus' smart watch [57] can be useful for Focal seizures as it has other sensors in addition to Galvanic Skin Response (GSR), for example EDA sensor and peripheral temperature, are just one of many sensors available in this device for researchers [221]. The Electro Dermal Activity (EDA) represents the electrical changes on the surface of the skin (not just for sweat). Although witnessed in some studies finding that EDA increases during GTCS were greater than during CPS (Complex Partial Seizure, now: focal seizure with loss of consciousness) nonetheless it is a useful sensor for this type of seizure [221].

The peripheral temperature sensor also has evidence for use in detection in non-convulsive seizure's (CPS) (focal) [221].

Although the Fitbit Ionic used in this study is not a conventional device for monitoring epilepsy, it can be adapted to detect seizures as demonstrated. It is a less expensive everyday 'patient friendly' option as opposed to EEG monitoring whereby the patient wears electrodes that are not comfortable: this is because the EEG-electrodes must be attached to the scalp which hampers the patient's movement making long-term home monitoring not feasible.

In this sense this less expensive, comfortable alternative to EEG monitoring can be especially useful for patients with non-epileptic events. During early analysis in this thesis many patient profiles (found in the collected 'anonymous patient profiles') where identified as having nonepileptic events and were categorised under "Non-Classified". Although no confirmed 'epilepsy' these patients are still suffering with seizure signs and symptoms: as observed below in 'HP5. Observation 1'(Figure 6.19). This patient has a non-epileptic shaking event at 23:03pm, the observation indicates heart-rate increase from 80 up to 109, the accelerometer also indicates rapid activity at the time of the shaking event. 
HP5. Observation 1

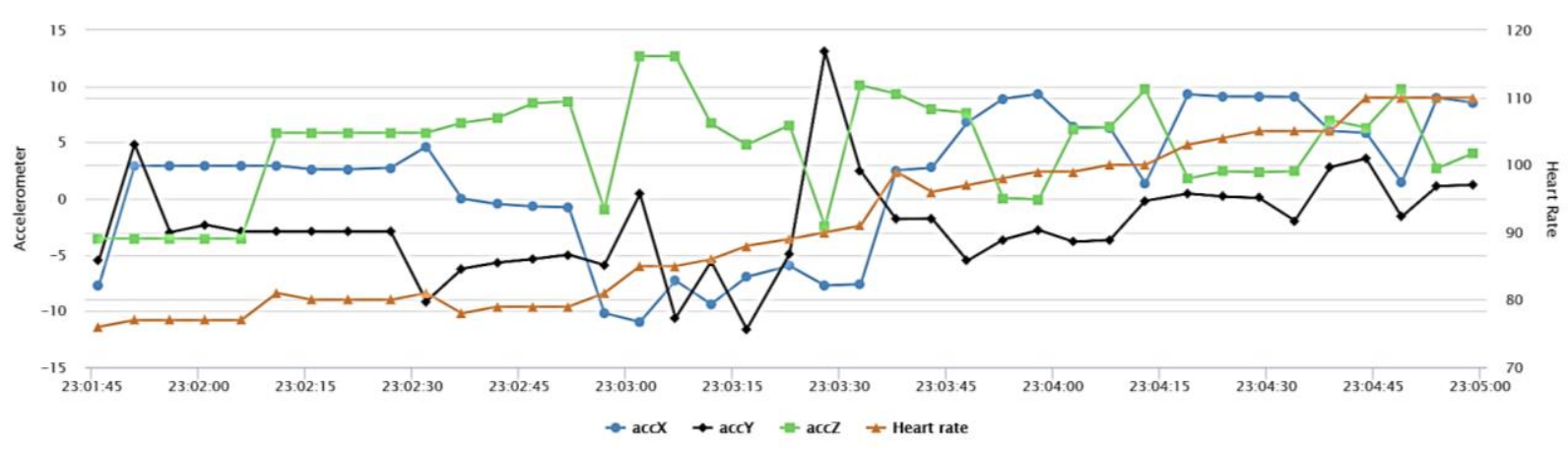

Figure 6.19. HP5. Observation 1

The non-epileptic patient HP5 can be compared with HP2 and HP3 whom have confirmed epilepsy yet HP2 and HP3 had no seizures during the observation time in this study, as seen below in 'Observation c HP2'(Figure 6.20) and 'Observation a HP3' (Figure 6.21).

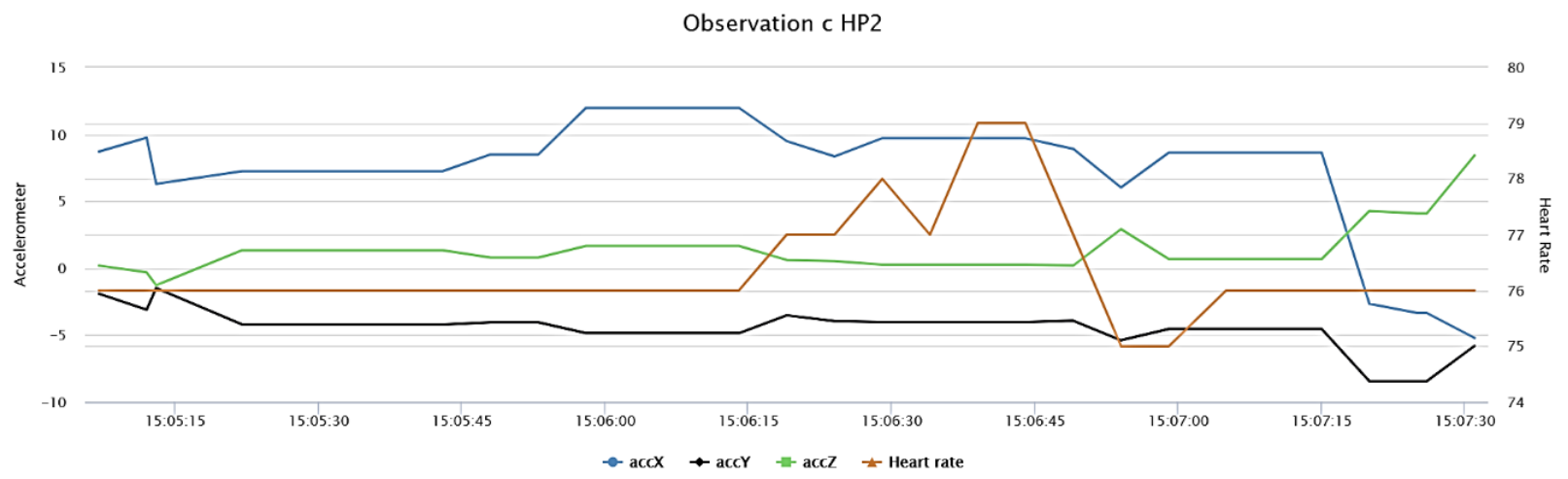

Figure 6.20. Observation c HP2

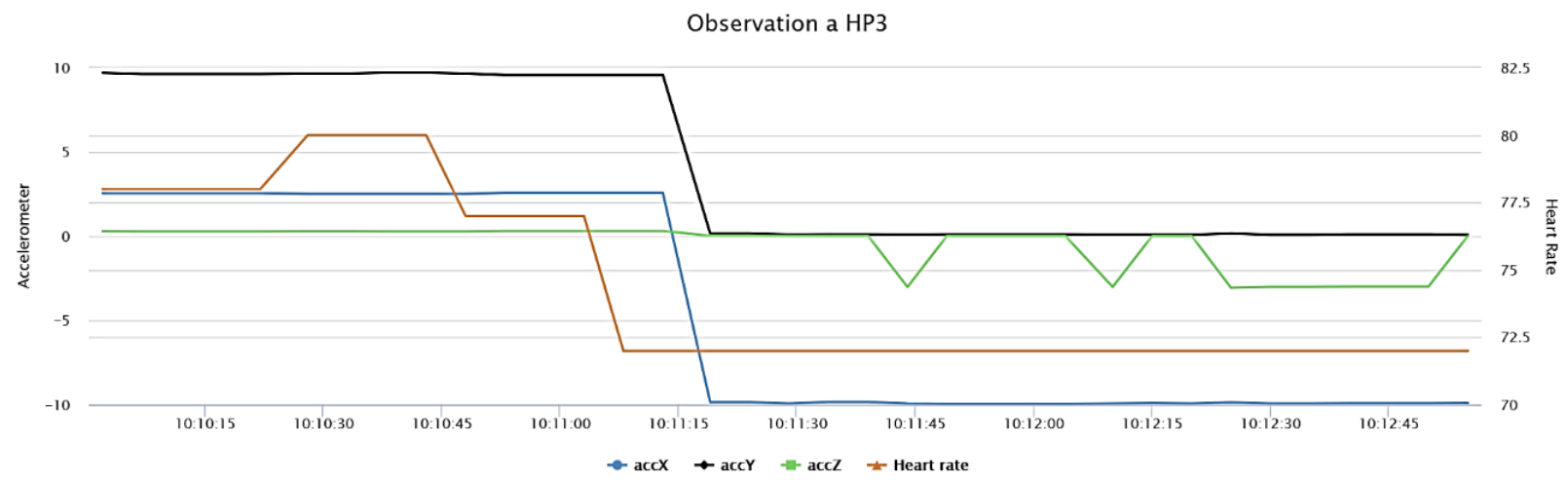

Figure 6.21. Observation a HP3

It is revealing how the accelerometer sensor behaves when there is no seizure (or rapid activity) compared to the movements witnessed in 'HP5. Observation 1' in Figure 6.19 above, hence the $\mathrm{X}, \mathrm{Y}$ and $\mathrm{X}$ axis are much smoother. This smoothness is further evidenced when HP2 is sleeping as in 'Observation a HP2' seen in Figure 6.22 below. 


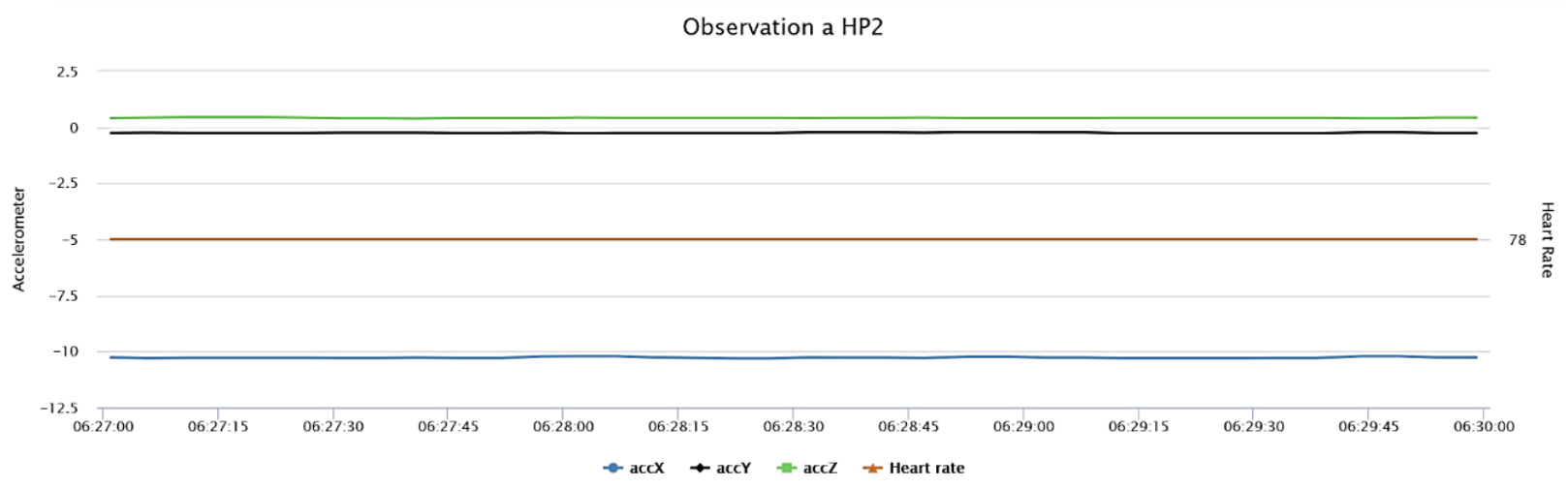

Figure 6.22. Observation a HP2

\subsection{Personalising Patient Profiles}

In principle different types of seizures are suitable for different sensor-based devices, for example S. Tiwari et al disclose that the beginning step towards choosing an appropriate seizure detection sensor device should be to find the main legitimate component character of the seizure [222].

Such parameters identified in this thesis are defined under the classes discovered in the Epilepsy Seizure Ontology (ESO): these components are used to evaluate an individual patients' profile. Once identified the Patients profile characteristics can be implanted into a "Cluster Group".

The 'Cluster Group' most corresponding to HP4 is 'Cluster Group 4' as identified in 'Table 5.8. Cluster Groups' Chapter 5 of this thesis. The predominant seizure type for HP4 is GTCS this is associated with 'shaking' (clonus) and in the case of HP4 other signs and symptoms are LOC, and automatisms associated with the other seizure type (FAS) accompanying HP4. Since the patient has LOC this lends itself to the use of an 'automatic detection' device as patient is unable to record their own seizure. The heart-rate and accelerometer sensor-based device is an appropriate recommendation for HP4 but also for GTCS seizure types, this is because there can be interruption to breathing indicating that cerebral oxygen saturation sensors could be useful monitoring tools and adopted as automatic prediction devices [3].

The 'Cluster Group' most corresponding to HP1 is 'Cluster Group 2' as identified in 'Table 5.8. Cluster Groups' in Chapter 5 of this thesis. This is because HP1 has FAS seizures, during the day-time only (diurnal), and seizures that occur 'multiple weekly: over 5'. This patient also has automatisms and sensations associated with FAS seizures and the dominant side is 'right'. The recommended sensor would be heart-rate as well as GSR to detect sweating during automatisms. As HP1 has a known history of 'Diurnal' seizures, dominantly on the right the sensors need only be worn during the day-time only and positioned upon the right of the body. 
The patients HP2 and HP3 both have the same seizure types, which are GTCS and FSIA. Both these seizure types correspond to Cluster Groups 2 and 4. It was common during earlier analysis in this study to find patients having more than one seizure type, and due to this the patient will reveal more complex characteristics, both patients have the key signs and symptoms: LOC (Loss of consciousness) and clonus (shaking) and the common signs and symptoms: automatisms. Both the patients have seizures which occur multiple weekly (over 5) and occur on both the left and right side of the body. The only specific difference in the characteristics of HP2 and HP3 is that HP2 has seizures that are 'Nocturnal' only (occur from sleep) whereas HP3 has both 'Nocturnal' and 'Diurnal' seizures. Based on this latter distinction the patients can be treated differently and hence 'personalisation' is realised.

The final patient: HP5 corresponds to 'Cluster Group 1' with 'Unclassified' as seizure type. Distinct in their diagnosis compared to other confirmed epilepsy patients HP5 can be however recommended a sensor-based device since they are having multiple weekly (over 5) events. A key sign and symptom is 'clonus' (shaking) from the 'left' therefore the accelerometer sensorbased device positioned on the left of the body could be helpful to detect these events. Furthermore, since these patients' only other symptoms are 'cognitive' (i.e. Memory impairment, déjà vu) other sensors would not be recommended.

\subsection{Volunteer Observations}

Prior to this experiment upon hospital patients, this study was conducted with 2 non-epileptic volunteers who were asked to undergo the testing and perform 'simulated seizures' in a controlled environment with the sensor-based device positioned on different parts of the body at different times of the day. This was to calibrate the main hospital patient experiments. The findings are discussed below. The full list of Volunteer Observations is in APPENDIX C, together with the simulated individual profile characteristics and criteria used for observations.

As can be seen from 'V1.Observation 1' in Figure 6.23 below, the first volunteer, with seizures occurring on the dominant right side of the body simulated a GTCS shaking from the right arm at the recorded time: $20.38 \mathrm{pm}$. The sensor-based device was worn on the right wrist. The 3 measurements on axis $\mathrm{X}, \mathrm{Y}$ and $\mathrm{Z}$ showed sudden movement and the heart-rate increased to its highest peak at 100. Before the seizure the heart-rate was steadier at 78-81, then after the seizure the heart-rate decreased to 80 . This suggested the sensors detected the simulated seizure correctly. 


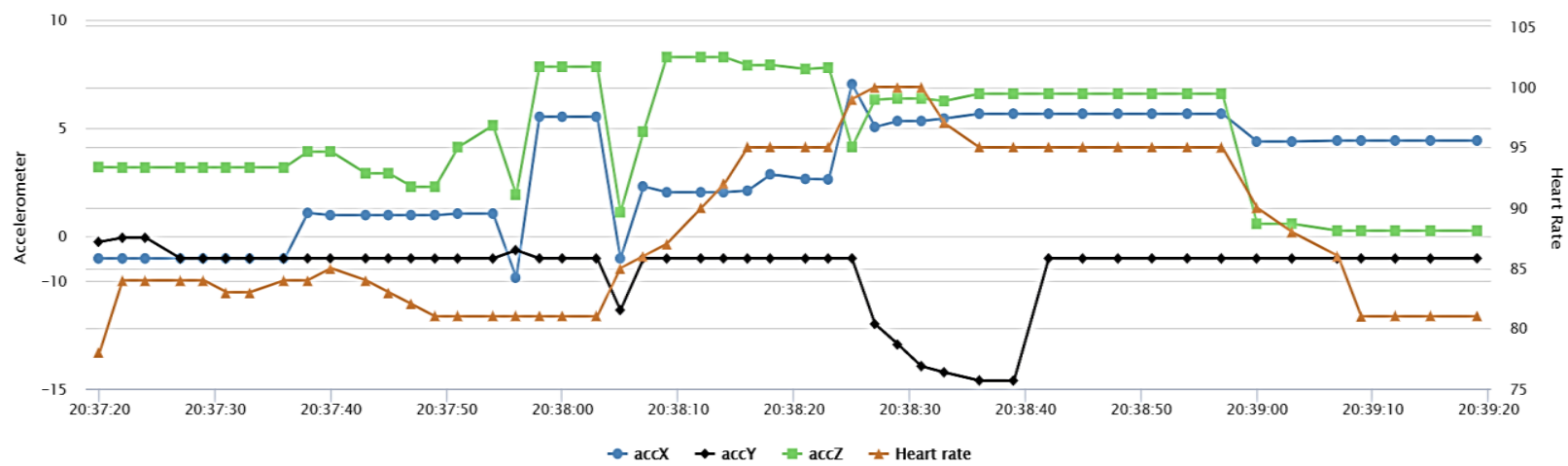

Figure 6.23. V1.Observation 1

When V1 simulated a seizure again from the right arm they placed the sensor-based device on the left wrist at the event time: 10.10am. As identified in 'V1. Observation 3' (Figure 6.24) the heart-rate shows an increase (78 to 123) yet the accelerometer axis is smooth. Similarly, in 'V1. Observation 4' (shown below in Figure 6.25) the volunteer placed the device on the 'nondominant' left leg and again the heart-rate increased dramatically but the acceleration generally smooth, although some movement on all 3 axis at the time: 09.47am of the event. Since 'some movement' was detected here a further test was performed with the device on the dominant right leg and here the difference is apparent, seen in V1. Observation 5 in Figure 6.26. This confirms the theory identified in earlier hospital patient observations that the sensor-based device position is paramount.

V1. Observation 3

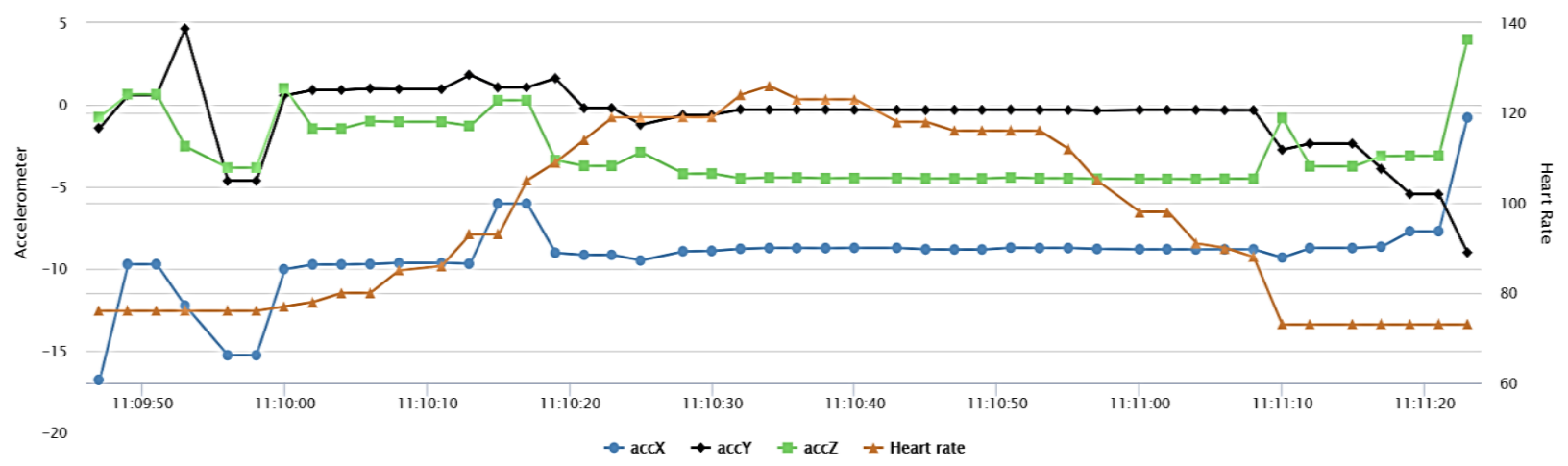

Figure 6.24. V1. Observation 3 


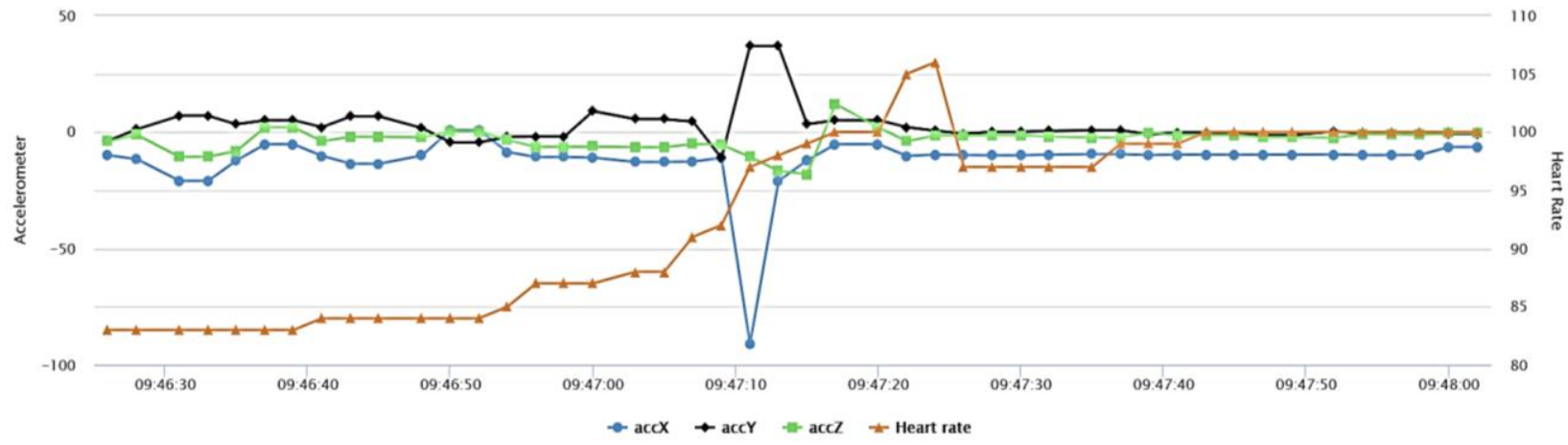

Figure 6.25. V1. Observation 4

V1. Observation 5

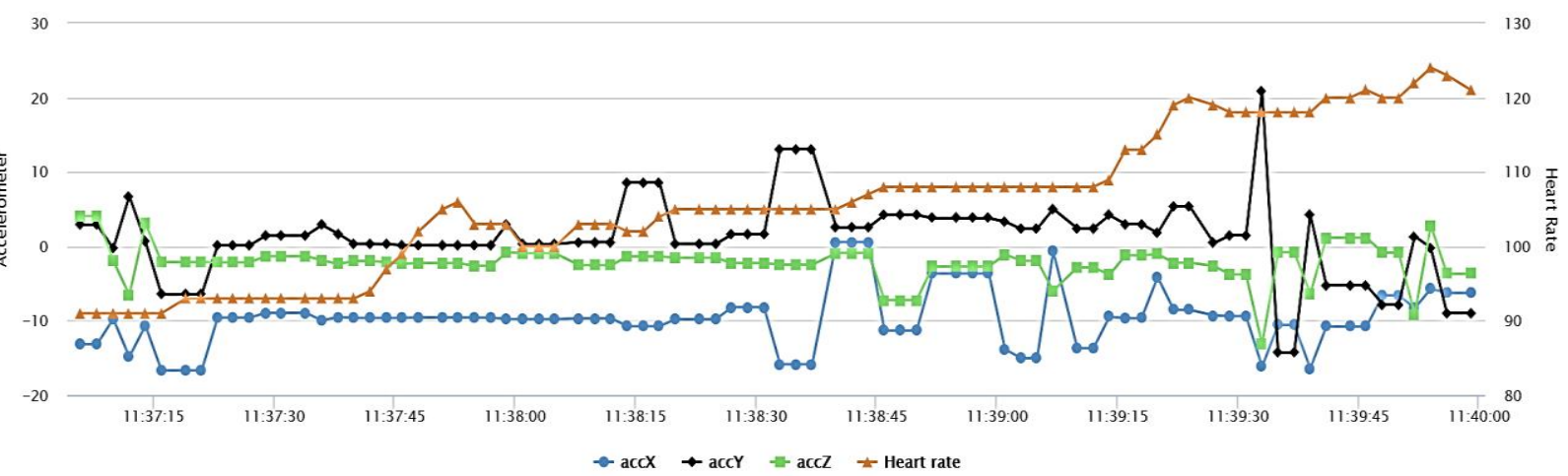

Figure 6.26. V1. Observation 5

For V2 the dominant side is left. It is evident when V2 placed the sensor-based device on the left leg during a simulated seizure at $22.14 \mathrm{pm}$ both the heart-rate and accelerometer sensors are reacting vigorously, seen in 'V2.Observation 1'(Figure 6.27). 


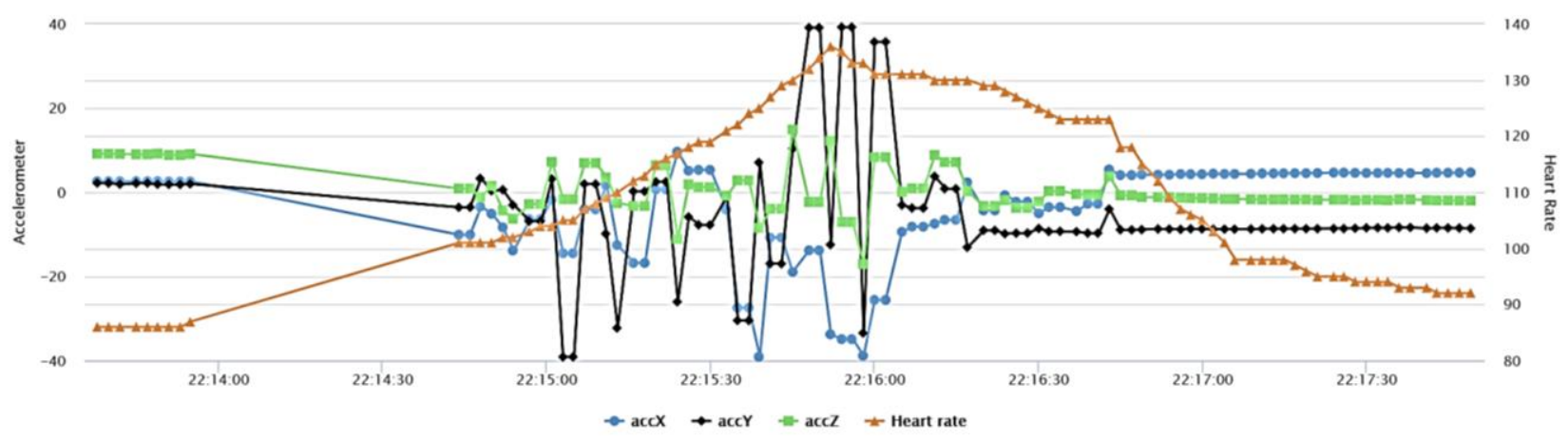

Figure 6.27. V2.Observation 1

Similar to V1 when V2 places the sensor-based device on the 'non-dominant' side (right leg) during a simulated seizure only the heart-rate sensor reacts, seen below in 'V2.Observation 1a'(Figure 6.28) again fuelling the theory that 'position' of the sensor-based device is paramount.

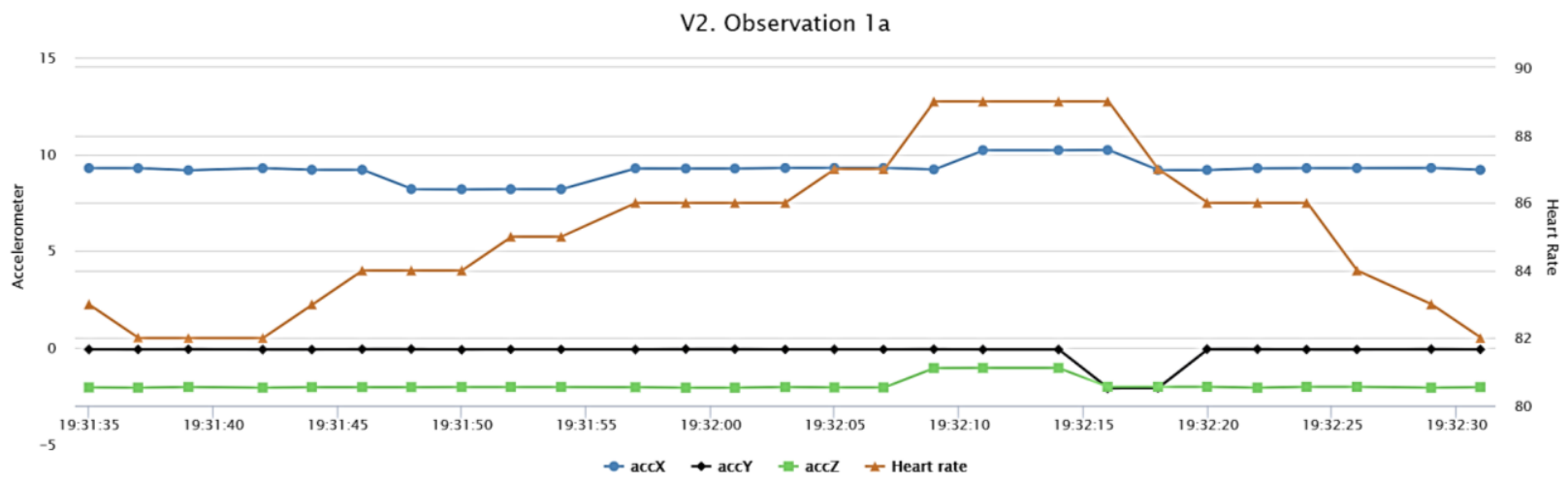

Figure 6.28. V2.Observation 1a

\subsection{Summary}

Detection of seizures using an everyday sensor-based device and data transfer to online database was successful. This presented evidence that remote monitoring of specific epilepsy patients' profiles with known characteristics can be improved. The comfortable sensor-based device with heart-rate and accelerometer provided accurate data and is a more dependable method than a patient's paper diary.

Difference was observed due to 'position' on the body of the sensor-based device, demonstrating that because of the known patient specific characteristics a personalised approach is achieved. Furthermore, it was discovered that the 'type' of sensor used is principal in its correspondence 
with a patients' particular 'seizure type' together with the particular associated signs or symptoms.

The sensors and techniques used in this experiment enable some assurance in long term remote monitoring. The use of such sensor-based device used in this experiment can reduce the frequency of visits to hospitals and improve daily management of epilepsy thus, these sensing techniques have shown that results can be achieved in the measurement of specific epileptic seizures based on observations.

As established through these experiments' timely detection along with known patient characteristics is one of the keys to monitoring epilepsy. 


\section{Chapter 7 Framework for Personalised Monitoring Plan}

\subsection{Introduction}

This chapter discusses the development of the Personalised Monitoring Plan framework (PMP). In the previous Chapter 6 experiments were performed to capture seizure data, obtained from sensors, which are positioned on different parts of the patient's body. This chapter discusses how results from this experiment are used to inform a PMP (Personalised Monitoring Plan) which recommends which sensor-based device to use based on those very individual, personal characteristics of a given patient. This chapter also corresponds to the methodology stage: 'I.V. PMP Framework \& Testing Stage' shown in Figure 3.1 Research Design Framework and addresses 'D2. Develop PMP Framework' and 'D3. Testing and Validation'.

The framework is made up of various components including all the data, resources, devices, tools (data analysis) and ontology used together. This proposed framework is a model for which doctors and healthcare professionals (HCP's) can use to assist in identifying which device they should recommend to the individual patient for remote monitoring.

This chapter also presents the testing of the PMP framework to ascertain its impact and effectiveness. It will focus on ensuring that the framework meets the requirements and fulfils the original study objectives. It will be evaluated using an epilepsy scenario and demonstrate that a 'personalised approach' is possible and ensure that it is a dependable and useful tool. A proposal for an IoT Epilepsy paradigm is also introduced in this chapter and as part of the solution further sensors are explored.

\subsection{Framework Philosophy and IoT}

In epilepsy, the most common and time-consuming patients to deal with correspond to individual strands of epilepsy, those patients that require long term monitoring assistance provided by doctors and caregivers. These highly varied kind of patient should be monitored specifically according to their symptoms, hence specific characteristics of each patient should be identified and medical treatment tailored accordingly [223] consequently paradigms are needed to personalise the information being described by both the condition of these patients each with 
their individual strand of epilepsy: (signs and symptoms etc.), and from the patient record held for these patients.

The PMP framework can employ aspects of the recent introduction of IoT technology which is available to aid epilepsy patients. An IoT-based model, namely the 'IoT Epilepsy Paradigm' is proposed and discussed in section 7.7.

Included in the PMP frameworks is the ESO ontology which was developed to organise the terms used to describe seizure type patients of which the output is the PPDL, (discussed in Chapter 4). This is used as a knowledge base for the personalisation of seizure type patients together with their signs and symptoms. The K-means Clustering Analysis result builds upon the ESO ontology and PPDL using its singular entities and in essence categorising them. This second personalisation process uses the personalised ontology of a patient and automatically transforms it into a seizure type category.

Consequently, the PMP framework integrates these two types of 'personalisation's':

$>$ The patient as the individual (the ESO ontology)

$>$ Patients in a category (K-means Clustering method)

The third tool of the PMP framework supports the decisions surrounding recommending the correct IoT sensor-based devices. The main purpose is to help HCP's decide which IoT Sensors to recommend for monitoring and which position on the patient's body.

The PMP framework ultimately allows users to provide a description of the 'seizure condition' of a single patient or a patient type, and to automatically obtain a PMP adjusted to the patient requirements. It can be difficult for a HCP to know how to use an ontology confidently since ontologies are rarely used beyond academic institutions [224] with this in mind the PMP framework was constructed to allow ease of use.

\subsection{PMP Framework}

The proposed framework consists of two features: the first being 'Personalisation' (based on this study) and the second is the anticipated 'Monitoring and Management', shown in pink and blue respectively in Figure 7.1. PMP Framework.

In this research, a patient record contains all the current medical information about the patient that may affect the immediate and short-term management of that patient. 
In order to provide a Personalised Monitoring Plan (PMP) described in this study only the 'seizure related' information of the patient is considered. This information is primarily composed of the patients' seizure types, signs and symptoms.

The 'seizure profiler' data and the 'cluster classifier' data plans are mutually related, and they define the knowledge base on which two knowledge personalisation procedures and one decision support process (Sensor Advisory) are implemented.

Therefore, given the condition of an epilepsy patient (or a seizure type patient), the PMP is used to personalise the medical knowledge available for that patient, discarding all the knowledge that is not related to that patient condition.

For example, in the PMP framework, the information from the patient record for how to deal with a patient profile is transformed and described instead with a single unified PMP (Personalised Monitoring Plan) whose information is more 'seizure' specific. The components in the framework are discussed in the next section and can be seen in Figure 7.1. PMP Framework 


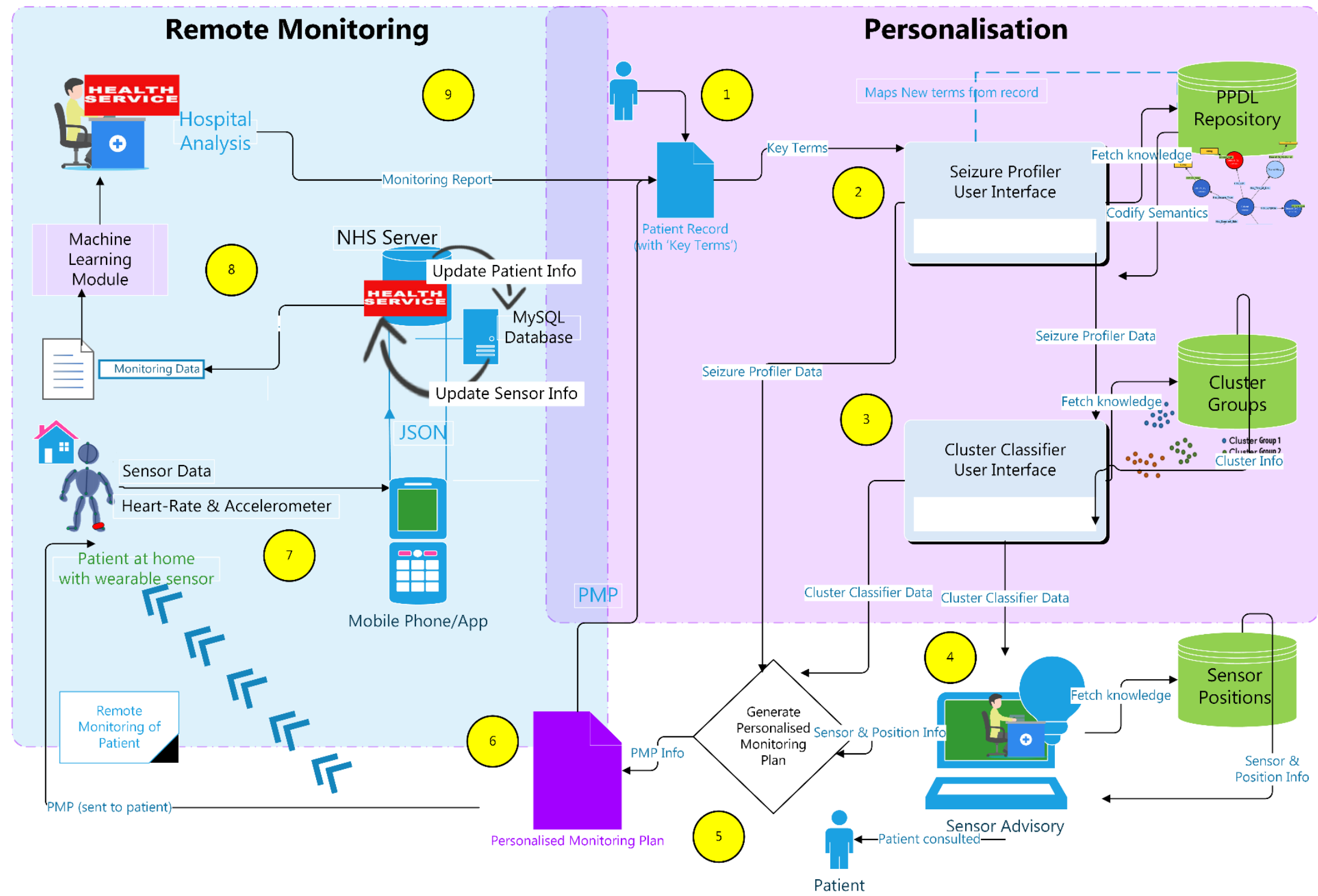

Figure 7.1. PMP Framework 


\subsection{Framework Components}

The framework is divided into 2 parts: 'Personalisation' and 'Remote Monitoring' both are discussed below together with the components contained in each.

\section{Personalisation}

Beginning with a patient record, this first step explores the PPDL repository to find possible feasible seizure types, signs and symptoms that have been observed by the HCP as 'key terms' in the patient record.

The knowledge about each single patient is personalised after a process of filtering upon entry to the Seizure Profiler component this is done to provide an evidence-based integrated description of the patient's particular seizure condition. To determine as much as possible the HCP's directly involved in the care of the patient, will incorporate the PPDL in within the record of the patient, and produce a new knowledge structure that can be incorporated later during in the 'Sensor Advisory' process in order to advise upon the patient's best sensorbased device and position.

The HCP will input these key terms to the 'Seizure Profiler' component.

This first personalisation process adapts the contents of the PPDL to the characteristics observed in the record of a given patient, automatically providing personalised seizure related data (through the Seizure Profiler component).

Knowledge from the PPDL repository is fetched founded on the user input i.e. a 'drop-down' list. Any new terms are mapped and added to the PPDL repository and updated.

The 'Seizure Profiler Data' is passed to the 'Cluster Classifier' component. The 'key terms' from the Seizure Related Profile Data are matched to the correct 'Cluster Group'.

The Cluster Classifier data is passed to the 'Sensor Advisory'.

\subsubsection{Sensor Advisory}

The clear purpose is to recommend a sensor-based device to an individual patient profile, to do this several factors had to be considered. Informed from discussions with clinicians and findings from experiments it was apparent that a complex set of profile combinations existed when addressing these essential device recommendation factors. 


\subsubsection{Clinician Advisory Factors}

As observed in "Clinician Advisory Factors" the number of seizure types considered to be the most common was ' 5 ', as demonstrated in Figure 7.2 below. These 5 random sample patient profiles that were developed during preliminary discussions with clinicians were chosen because they were patients which were considered to have 'the most common' type of seizure and it was agreed that 5 represented a good range.

The 3 other factors that were considered aspects when influencing the decision for the recommended sensor-based device per patient profile from the clinicians are listed below and shown in Figure 7.2

$\begin{array}{ll}\text { - } & \text { dominant side } \\ \text { - } & \text { limb indicator } \\ & \text { signs and symptoms }\end{array}$

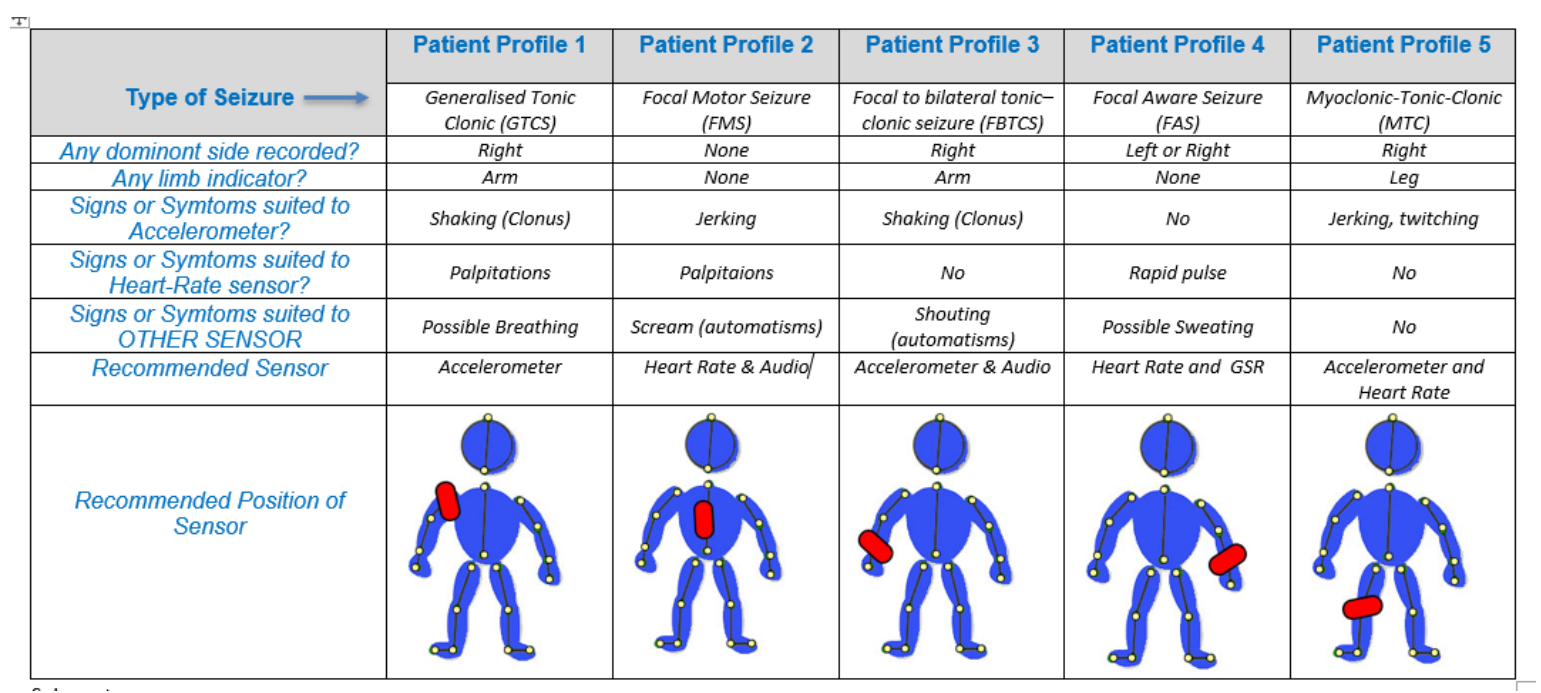

Figure 7.2. Clinician Advisory Factors

However, the ultimate factor proposed from the clinicians to make the decision was the "seizure type". Informed from discussions this proposal can be observed in Table 7.1. Matching Aspect whereby each of the main seizure types are matched with a useful sensorbased device, these matches were confirmed accurate and realistic as witnessed in the experiments conducted in the previous Chapter. Furthermore, influenced by discoveries highlighted during analysis of the conducted experiments seizure type ' $A$ ' (which is GTCS) has additional sensors assigned: 'heart-rate' \& 'oxygen' sensor.

Table 7.1. Matching Aspect

\begin{tabular}{|l|l|l|l|l|l|}
\hline $\begin{array}{l}\text { Seizure } \\
\text { Type }\end{array}$ & A: GTCS & B: FMS & C: FBTCS & D: FAS & E: MTC \\
\hline $\begin{array}{l}\text { Sensor- } \\
\text { based } \\
\text { Device }\end{array}$ & $\begin{array}{l}\text { 1. Accelerometer } \\
\text { and or/ Heart-rate } \\
\text { and oxygen }\end{array}$ & 2. Heart-Rate & $\begin{array}{l}\text { 3. Accelerometer } \\
\text { \& Audio }\end{array}$ & $\begin{array}{l}\text { 4. Heart- } \\
\text { Rate and } \\
\text { GSR }\end{array}$ & $\begin{array}{l}\text { 5.Accelerometer } \\
\text { and Heart-rate }\end{array}$ \\
\hline
\end{tabular}


Matching the seizure type to the device based on the 'Matching Aspect' table is an easy way to recommend a device to a patient, however all the other factors and variants must be considered.

Firstly the "dominant side" and "limb indicator" factors were considered. When applying these factors to patient profiles per 'seizure type' a wide variety of alternative combinations are revealed. These factor infused profiles are shown below in Table 7.2.

Table 7.2. Dominant Side and Limb Factors

\begin{tabular}{|l|l|l|l|l|}
\hline $\begin{array}{l}\text { Seizure Type A } \\
\text { with 6 variants }\end{array}$ & $\begin{array}{l}\text { Seizure Type B } \\
\text { with 6 variants }\end{array}$ & $\begin{array}{l}\text { Seizure Type C } \\
\text { with 6 variants }\end{array}$ & $\begin{array}{l}\text { Seizure Type D } \\
\text { with 6 variants }\end{array}$ & $\begin{array}{l}\text { Seizure Type E } \\
\text { with 6 variants }\end{array}$ \\
\hline A right arm & B right arm & C right arm & D right arm & E right arm \\
\hline A right leg & B right leg & C right leg & D right leg & E right leg \\
\hline A left arm & B left arm & C left arm & D left arm & E left arm \\
\hline A right leg & B left leg & C left leg & D left leg & E left leg \\
\hline A both sides & B both sides & C both sides & D both sides & E both sides \\
\hline A both limbs & B both limbs & C both limbs & D both limbs & E both limbs \\
\hline
\end{tabular}

As observed (in Table 7.2) 30 different combination profiles exist demonstrating the range and high number of variants when the dominant side and limb indicator are applied to the seizure type. If the 'signs and symptoms' factors are also added, it is implicit that the amount of patient profiles grows even further. Consequently, the driving questions here are:

"how can all these profile combinations be represented with their matching device and positions?"

"what mechanism can be put in place to assist HCPs in making the decision on which device and position to recommend to an individual patient profile.?"

These decisions are made by using the findings in this study and henceforth discussed. One approach is to form a data flow chart using a linear process yet this could not capture all the profile combinations with their multi-variants. Figure 7.3. Linear Process Limitations shows an example of decisions in a flow chart that can be made at the point of a recommendation assessment for a given patient profile that has a known 'seizure type' (demonstrated by rectangle process box). This linear process reveals limitations, one such being the fact that seizure 'symptoms' are not carefully considered and also lacks the injection of the new knowledge found in this research about cluster groups. Whilst straight forward decisions can be made about matching the correct device with a given seizure type (those represented by the diamond symbol) and likewise the dominant side and limb factor, the process is too shallow. The next sections address these limitations and seek to output such a result that would reveal truly all-embracing recommendations. 


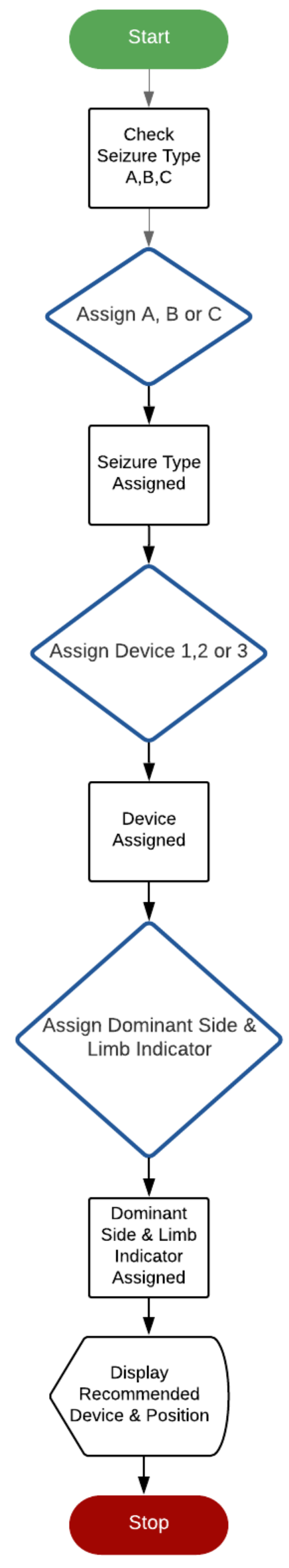

Figure 7.3. Linear Process Limitations 


\subsubsection{Matching the Correct Device to the Cluster Groups}

Whilst addressing the question of matching the correct device with each of the 6 cluster groups identified in Chapter 5 several issues came to light. The theory derived from the clinicians previously discussed was to simply match the seizure type to the device, as seen previously in Table 7.1. Matching Aspect.

Yet if a given patient profile has seizure type B (FMS) does this mean they should automatically be assigned 'Device 2' (heart-rate sensor)? If this is the case, then other factors (shown in green below) have no bearing and become irrelevant. A snapshot in Figure 7.4 (Cluster Group and Device Analysis 1) below demonstrates the problem thus providing an overview of the challenge to match the correct seizure type with the correct device and include 'dominant side' and 'limb factors' and 'signs and symptoms': Each of the 6 cluster groups are indicated by colour, i.e. Cluster 0: blue line, Cluster 1:orange line etc.

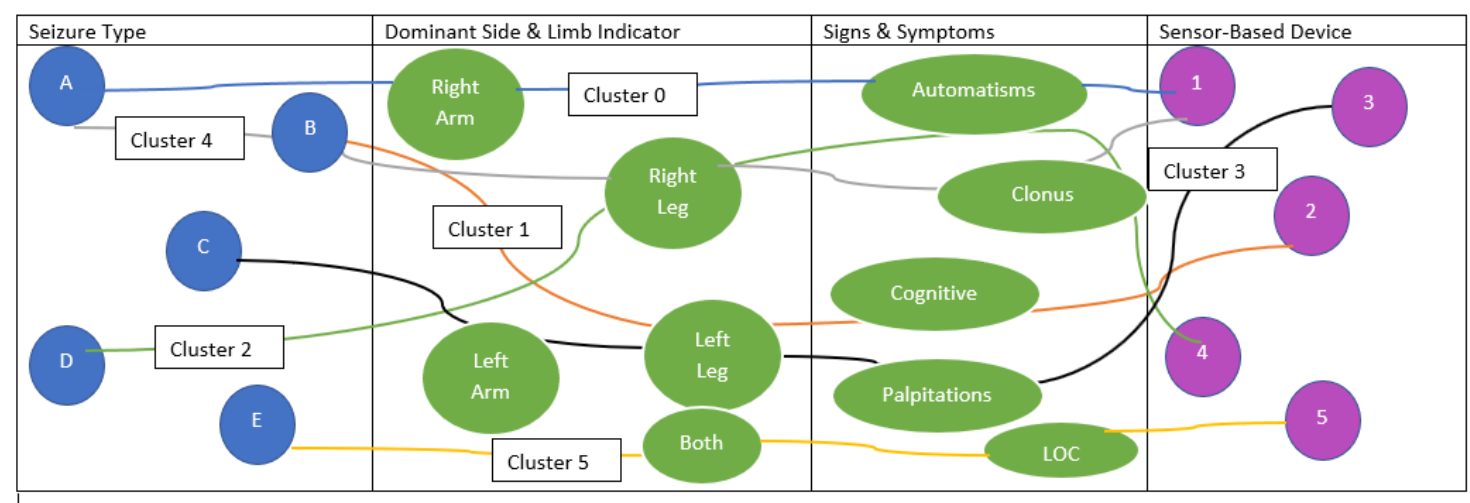

Figure 7.4. Cluster Group and Device Analysis 1

Yet as informed from the experiment results it was observed that it was the 'Signs \& Symptoms' that had the greatest influence upon the decision for example during the experiments in this study the patient, namely: 'HP1' with 'Focal' seizure type was found to have 'heart-rate changes' during their seizure, this symptom reveals more about the individual than the actual 'seizure type' did since this type of seizure was not originally considered for the heart-rate sensor as observed in Figure 7.2. Clinician Advisory Factors, 'Patient Profile 3' with a Focal type seizure. The 'dominant side' \& 'limb indicator' factors can have no bearing upon the type of device to be recommended only the 'position': yet as discovered during experiments: 'position is paramount' but nevertheless this is still not a factor influencing device decision. This factor instead should be treated as essential accompanying factor and thus as an "addon". With this in mind this addon is removed from the analysis as seen in Figure 7.5 below: 


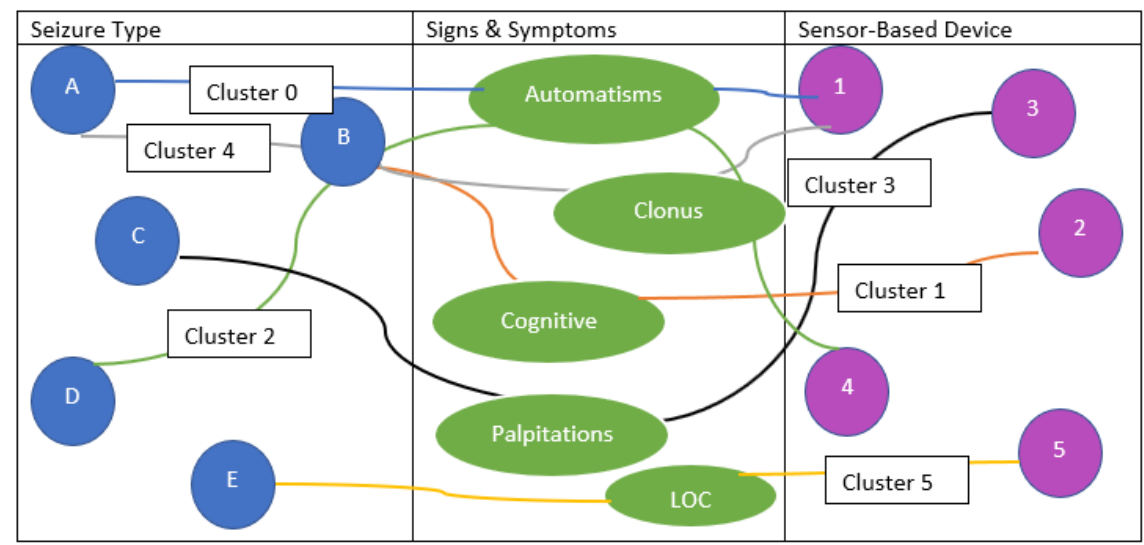

Figure 7.5. Cluster Group and Device Analysis 2

Now that this factor has been removed the next focus is on how the 'seizure type' has bearing upon the decision. Upon returning to the example i.e. "a patient profile with seizure type $B$ (FMS)", the assumption is applied that this profile should automatically be assigned 'Device 2' (heart-rate sensor). Yet, what if this particular patient profile has 'sweating' and 'shaking' too? So therefore, should they not also be assigned the 'Galvanic Skin Response' sensor?

The original assumption from the clinicians that a device can be recommended based upon seizure type is not sound because as demonstrated "signs and symptoms" play a more superior part in the decision. With this in mind the "matching aspect of seizure type with device" is removed (as seen in Figure 7.6. Cluster Group and Device Analysis 3) and just the "signs and symptoms" remain as the prime factors influencing the choice of device. This makes the "seizure type" factor irrelevant. Removing the "seizure type" from the decision is further affirmed due to observations derived from the experiments on the hospital patients whereby there existed patient profiles that often had more than one seizure type. As this holds true how could 'multi' seizure types correspond to a device? Similarly, a patients 'signs and symptoms' can vary, especially for an "unclassified" seizure type: as witnessed during experiments in Chapter 6 whereby HP5 revealed 'shaking' symptoms' yet another 'unclassified' seizure type patient may reveal 'palpitation' symptoms only. Therefore, it is only the 'signs and symptoms' that can influence the device choice, and the next step is to see how features of the sensor-based device correspond to particular signs and symptoms. Ultimately, the decision resides on what features the sensor-based device can offer, in essence: how the actual sensors can match the signs and symptoms. 


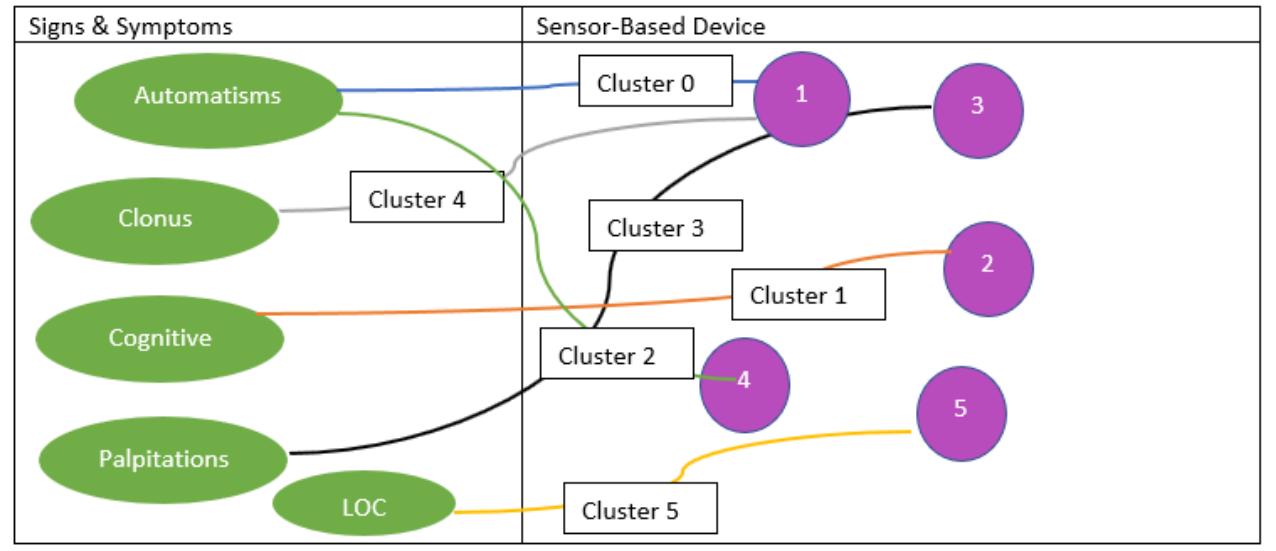

Figure 7.6. Cluster Group and Device Analysis 3

\subsubsection{Assigning the Sensors}

Informed from the findings a method has been put in place to recommend sensors to an individual patient profile. The foundation for this is based upon the following logic, shown in Table 7.3. Assigning the Sensors', here each of the 6 'Cluster Groups' is assigned a sensor or 'sensors', (shown in blue). Then according to how the individual 'signs and symptoms' for that cluster correspond to available sensors each sign and symptom is labelled: i.e. given a patient with 'myoclonus' (full body shaking) the sensor will be the 'accelerometer'(labelled 'A'), given a patient with 'Automatisms' the sensor will be 'heart-rate' (labelled 'B') and so on. Some symptoms do not correspond to a sensor since there are not always available sensors for symptoms i.e. LOC (Loss of consciousness), henceforth these symptoms are labelled 'NS' (no sensor). The table below Table 7.3. Assigning the Sensors' demonstrates this logic.

\begin{tabular}{|c|c|c|c|c|c|c|}
\hline Cluster & Cluster 0 & Cluster 1 & Cluster 2 & Cluster 3 & Cluster 4 & Cluster 5 \\
\hline \multirow[t]{2}{*}{$\begin{array}{l}\text { Key Sign } \\
\& \\
\text { Symptoms }\end{array}$} & $L O C \mathrm{NS}$ & $L O C \mathrm{NS}$ & Sensations C & None & $\begin{array}{l}\text { Myoclonus A } \\
\text { Bilateral Clonus A }\end{array}$ & Bilateral Clonus A \\
\hline & $\begin{array}{l}\text { Urinary } \mathbf{E} \\
\text { Incontinence } \mathbf{E}\end{array}$ & & & & $L O C \mathrm{NS}$ & $L O C \mathrm{NS}$ \\
\hline $\begin{array}{l}\text { Common } \\
\text { Sign \& } \\
\text { Symptoms }\end{array}$ & Automatisms B & $\begin{array}{l}\text { Cognitive NS } \\
\text { Automatisms B }\end{array}$ & Automatisms B & Automatisms B & $\begin{array}{l}\text { Sensory D } \\
\text { Cognitive NS }\end{array}$ & Automatisms B \\
\hline $\begin{array}{l}\text { Sensors } \\
\text { Assigned }\end{array}$ & $\begin{array}{l}\text { Heart-Rate \& } \\
\text { Bladder Sensor }\end{array}$ & $\begin{array}{l}\text { Heart-Rate } \\
\text { Sensor }\end{array}$ & $\begin{array}{l}\text { GSR \& Heart- } \\
\text { Rate Sensor }\end{array}$ & $\begin{array}{l}\text { Heart-Rate } \\
\text { Sensor }\end{array}$ & $\begin{array}{l}\text { Accelerometer \& } \\
\text { Audio Sensor }\end{array}$ & $\begin{array}{l}\text { Accelerometer \& } \\
\text { Heart-rate }\end{array}$ \\
\hline \multicolumn{7}{|c|}{$\begin{array}{l}\text { Key: } \\
\text { NS=No Sensor }\end{array}$} \\
\hline
\end{tabular}

Table 7.3. Assigning the Sensors

The next step demonstrates how the decisions are 'processed' in the form of a decision tree, shown in Figure 7.7. Sensor Decision Tree. (The complete decision tree is shown at the top 
and there is an 'expanded view' beneath of a random portion of the tree for added visual clarity, together with a key for each abbreviation used.)

The decision tree verifies the clusters to be correct since every combination of the 5 sensors (for each symptom) is presented in the 'Sensor Decision Tree', so if each cluster runs its pathway through the decision tree it confirms the most suitable sensors for that particular cluster, as indicated in Table 7.3 above. In addition to the 6 cluster groups, every other outcome (or eventuality) is offered based upon the identified signs and symptoms from the cluster groups. To illustrate the concept in the 'Sensor Decision Tree' the following are queried: i.e.

does the patient have the symptom clonus?

if 'yes' go to next question:

does the patient have the symptom automatisms?

if 'no' go to next question:

does the patient have the symptom sensations?

if 'yes' go to next question:

does the patient have the symptom sensory?

if 'yes' go to next question:

does the patient have the symptom urinary?

if ' $n o$ ' go to next question:

then assign sensors $A, C, D$ 


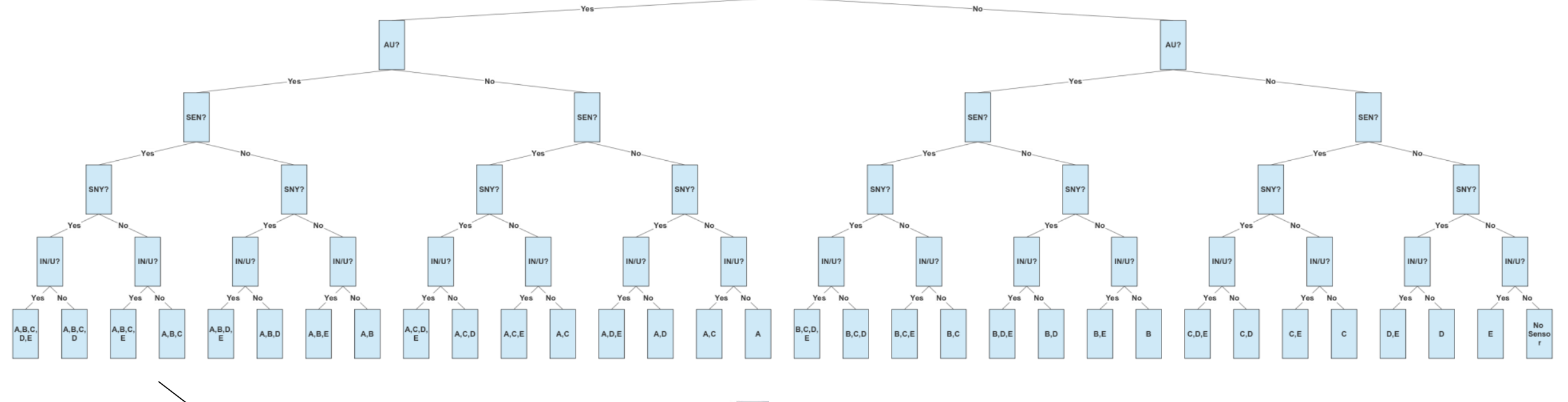

Expanded View
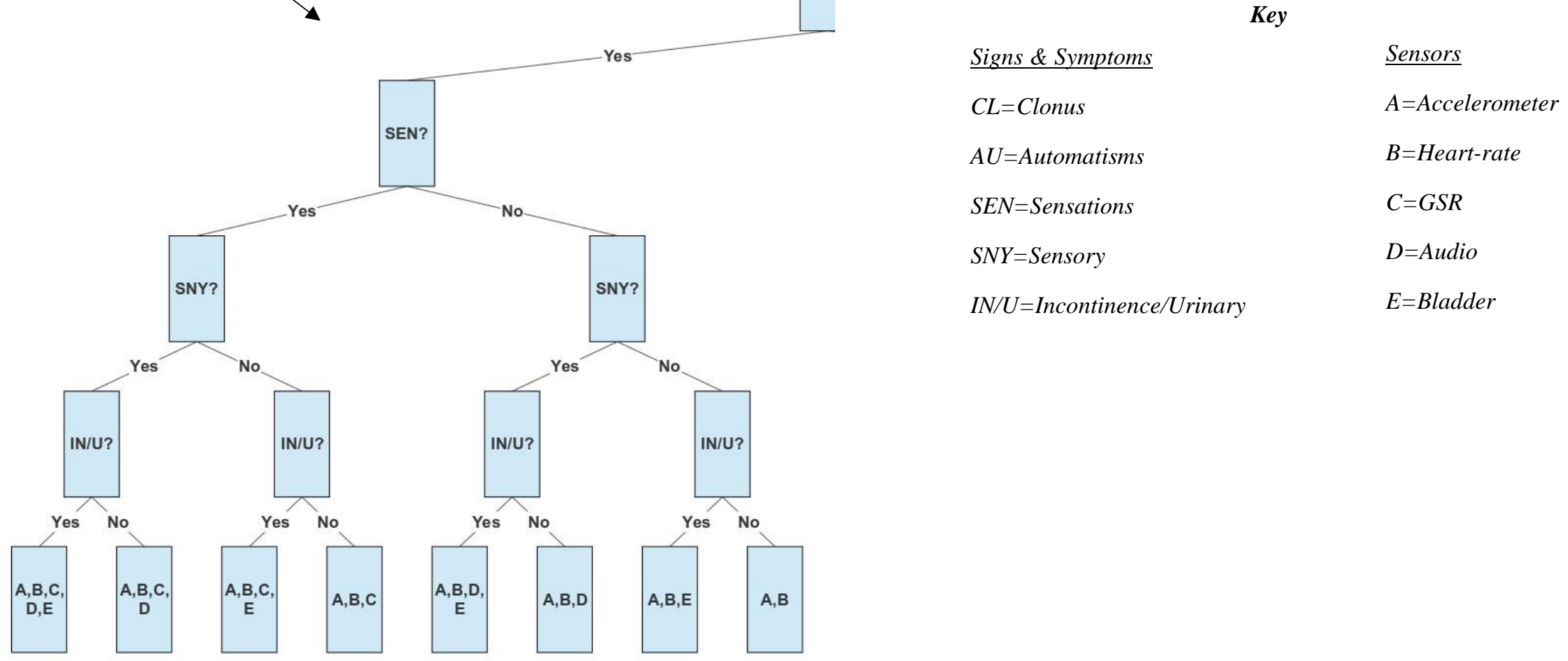
Based upon the evidence that particular signs and symptoms match with a sensor, shown in this approach, it can be projected that future epilepsy patients displaying epilepsy symptoms in a similar manner will require the same sensor. In this sense a future goal is to use the decision tree as a training model that can be used to predict the target variable, i.e. the sensor, by learning simple decision rules inferred from prior training data [225].

The decision tree presented here is easy to understand, users can relate to its theory, its graphical representation is intuitive. For future data exploration it is expected more variables will be added such as signs/symptoms, sensors and positions, and therefore the decision tree can help identify the most significant variable.

Decision trees can be used for classification and regression, and algorithms such as CART exist to grow the tree to predict the outcome in each leaf node the average outcome of the training data in this node is used [226]. Using the approach described in this thesis other health domain experts and researchers can take this to the next level.

\section{Generate the PMP}

The next component in the PMP framework is to generate the PMP. Now that the chosen sensor has been assigned together with the 'addon' position information, it is sent to the part of the framework which will generate the PMP (Personalised Monitoring Plan).

In summary the three datasets that are passed to the 'Generate PMP' component during the process in the framework are:

$>$ Seizure Profiler Data

$>$ Cluster Classifier Data

$>$ Sensor \& Position Information

These three datasets will form the PMP, a sample is shown in Figure 7.8. PMP Sample. 
Personalised Monitoring Plan

Patient Profile Number: 56

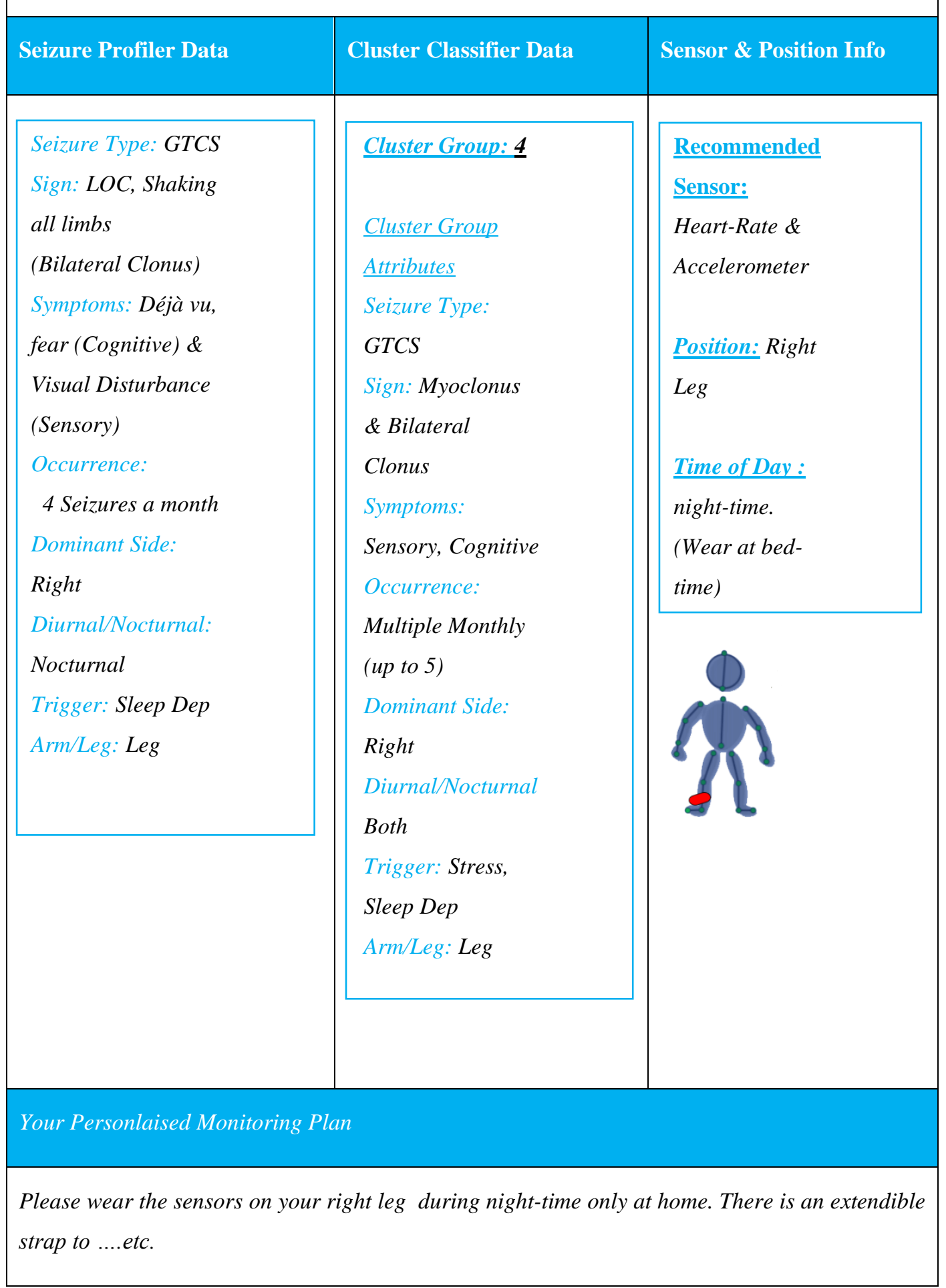

Figure 7.8. PMP Sample 


\section{$>$ Remote Monitoring and Management}

The new knowledge gained in the PMP framework will be updated in the patient record to help general practitioners in their daily tasks of visiting patients, follow-up, diagnosis, planning of treatments and foreseeing changes. The PMP is sent with the patient to their home. The patient then receives the PMP together with the monitoring IoT sensor-based device containing the two sensors heart-rate and accelerometer as described during the experiment investigation and design. The patient wears the device and the monitoring data is then sent from the device to the mobile phone from which it's paired, and from there to the Health Service server.

The proposed Health Service web server connects the data between the mobile phone, using JSON. The web server helps to save the data, to the MySQL database server also located in the hospital. The monitoring data can then be exported directly from the database.

The app sends the 'monitoring data' from patient to the Health Service server to process and then the monitoring data is displayed shown during the experiment design, see APPENDIX A 'Backend Monitoring'. JSON (JavaScript Object Notation) is used as the data format to exchange information between client and server. JSON is a lightweight data-interchange format, JSON uses the text format which is completely independent from different programming languages. Furthermore, it not only supports several programming languages but has a satisfactory transmission and parsing speed [227] [228].

Finally, an HCP will receive the 'monitoring data' from the Health Service server for the given patient, this data will then be analysed. To analyse the monitoring data there will be a built in 'machine learning module' to determine seizure detection or not. This learning module uses training algorithms and replaces the need for humans by using a machine learning approach. Due to limitations in this study this module has not been built but instead is proposed at this stage.

\subsection{PMP Framework Loop and Maintenance}

With this PMP framework, the PPDL can be directly maintained (and extended) by HCP's. The framework has the flexibility, (as the ontology grows with new seizure related concepts), to deal with the mounting diversity of seizure type patients. Therefore, the PMP framework is to some extent reliant on the integration of new knowledge in the PPDL. As the HCP of the PMP Framework confirms the recommendations, the information about the patient and the advice may change and this new information can cause the PMP Framework to continue providing new suggestions to the HCP.

This loop stops either when the framework is not able to provide new recommendation or when the HCP considers that the current condition of the patient is perfectly represented by 
the recommendation. At any point, the PMP for the patient is left to the HCP who is using the framework.

Consequently both the personalised 'seizure related data' and the 'cluster classifier data' of a patient may evolve as the patient condition changes (i.e., when the information about the patient changes in the patient record of that patient) or as a result of the application of the PMP Framework to find out new 'seizure type' knowledge about the patient in question. These evolved datasets can be continually stored as part of the record of that patient.

\subsection{Use Case Evaluation}

Here the evaluation of the PMP framework in epilepsy scenarios is undertaken. Two different scenarios each with different patient profiles will be tested by showing their respective inputs and outputs. The result will help determine how effective the developed framework can be used as a tool for recommending the IoT device to an epilepsy individual patient. The steps (1-9) taken in each scenario throughout the framework are discussed and can be seen in 'Figure 7.1. PMP Framework' numbered in yellow.

\subsubsection{Scenario 1}

\section{Use Case Name: Scenario 1}

Use Case Description: In this scenario an HCP takes a sample patient through each of the steps in the PMP framework

Actors: Patient Profile 56 (with an existing patient record)

Triggers: The patient indicates that he/she wants to have an IoT sensor-based device to remotely monitor their epilepsy.

Preconditions: The patient has known frequent seizures that have been recorded in the patient record.

\section{Post-conditions:}

i. The patient will receive a PMP (Personalised Monitoring Plan)

ii. The patient will receive a recommended IoT sensor-based device that they will place on position on their body.

iii. The patient's seizures will be monitored by the NHS.

\section{Normal Flow:}


1. The patient will indicate that he/she wants to have an IoT sensor-based device to remotely monitor their epilepsy and provides consent for the HCP to access their patient record.

2. The HCP using the PMP framework will explore the PPDL repository (see Step ' 2 ' in Figure 7.1. PMP Framework) to find possible feasible seizure types, signs and symptoms that have been observed by the HCP as 'key terms' in the patient record. See Figure 7.9. Patient Record Sample 1.

\begin{tabular}{|l|l|}
\hline Hospital Number:\#\#\# & $\begin{array}{l}\text { Referring } \\
\text { Clinician:\#\#\# }\end{array}$ \\
\hline Test Type: EEG TELEM & Relevant Medical \\
\hline $\begin{array}{l}\text { CLINICAL HISTORY: } \\
\text { event:(Generalised Tonic Clonic, } 4 \text { in a month, usually } \\
\text { occur when lack of sleep, and when asleep, can have a } \\
\text { warning during the night like increased heart-rate, } \\
\text { feeling strange (happened before), visual disturbance } \\
\text { unable to respond. Partner reports full body-jerking, } \\
\text { mainly begins on right leg. Patient feels tired and } \\
\text { confused next day. }\end{array}$ & Family Heview \\
\hline
\end{tabular}

Figure 7.9. Patient Record Sample 1

The patient record is personalised after a process of filtering upon entry to the 'Seizure Profiler' component (Figure 7.10. Seizure Profiler). 


\section{Seizure Profiler}
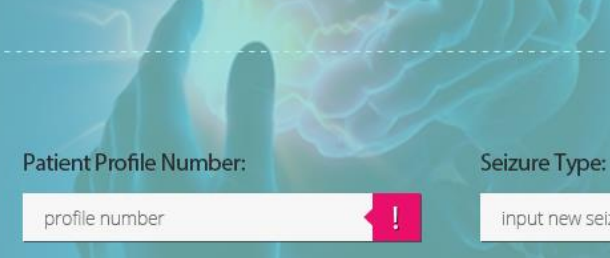

Seizure Occurances:

Once Daily

Multiple Daily $\checkmark$

Once Weekly

Dominant Side:

Left

Right

$$
\text { - }
$$

Dirunal/Nocturnal:

Dirumal

Nocturnal

Trigger:

Sleep Deprivation

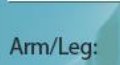

Arm/Leg:

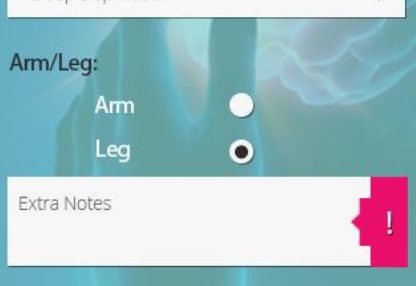

\section{Seizure Type:}

input new seizure type

select seizure type

FMS

FBTCS

GTCS

MTC

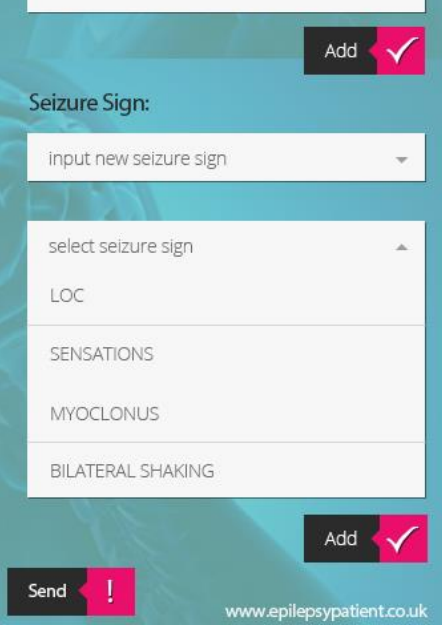

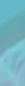

-
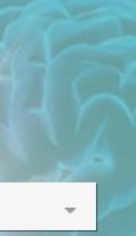

Figure 7.10. Seizure Profiler

Automatic personalised seizure related data from the Seizure Profiler is fetched. The output is the Seizure Profiler Data. The patient output for Patient profile 56 is revealed here: Table 7.4. Sample Seizure Profiler Data. Keywords from the patient record have been incorporated and terminology updated.

\begin{tabular}{|c|c|c|c|c|}
\hline Patient Profile & $\begin{array}{l}\text { Seizure } \\
\text { Type }\end{array}$ & Key Signs & Symptom & Occurrence \\
\hline 56 & GTCS & $\begin{array}{l}\text { LOC, } \\
\text { Bilateral Clonus } \\
\text { Palpitations }\end{array}$ & $\begin{array}{l}\text { Cognitive } \\
\text { Sensory }\end{array}$ & $\begin{array}{l}\text { Multiple seizures (up } \\
\text { to } 5 \text { per month }\end{array}$ \\
\hline Dominant Side & \multicolumn{2}{|c|}{ Diurnal/Nocturnal } & Trigger & Arm/Leg \\
\hline Right & \multicolumn{2}{|c|}{ Nocturnal } & Sleep Dep & Leg \\
\hline
\end{tabular}

Table 7.4. Sample Seizure Profiler Data 56 
3. The PMP framework passes the 'Seizure Profiler Data' to the 'Cluster Classifier' component. The PMP framework matches the 'key terms' from the 'Seizure Related Profile Data' to the correct 'Cluster Group' i.e. then, given the sample in Table 7.4. Sample Seizure Profiler Data 'Patient profile 56' is matched to 'Cluster Group 1', see Figure 7.11. Cluster Classifier.

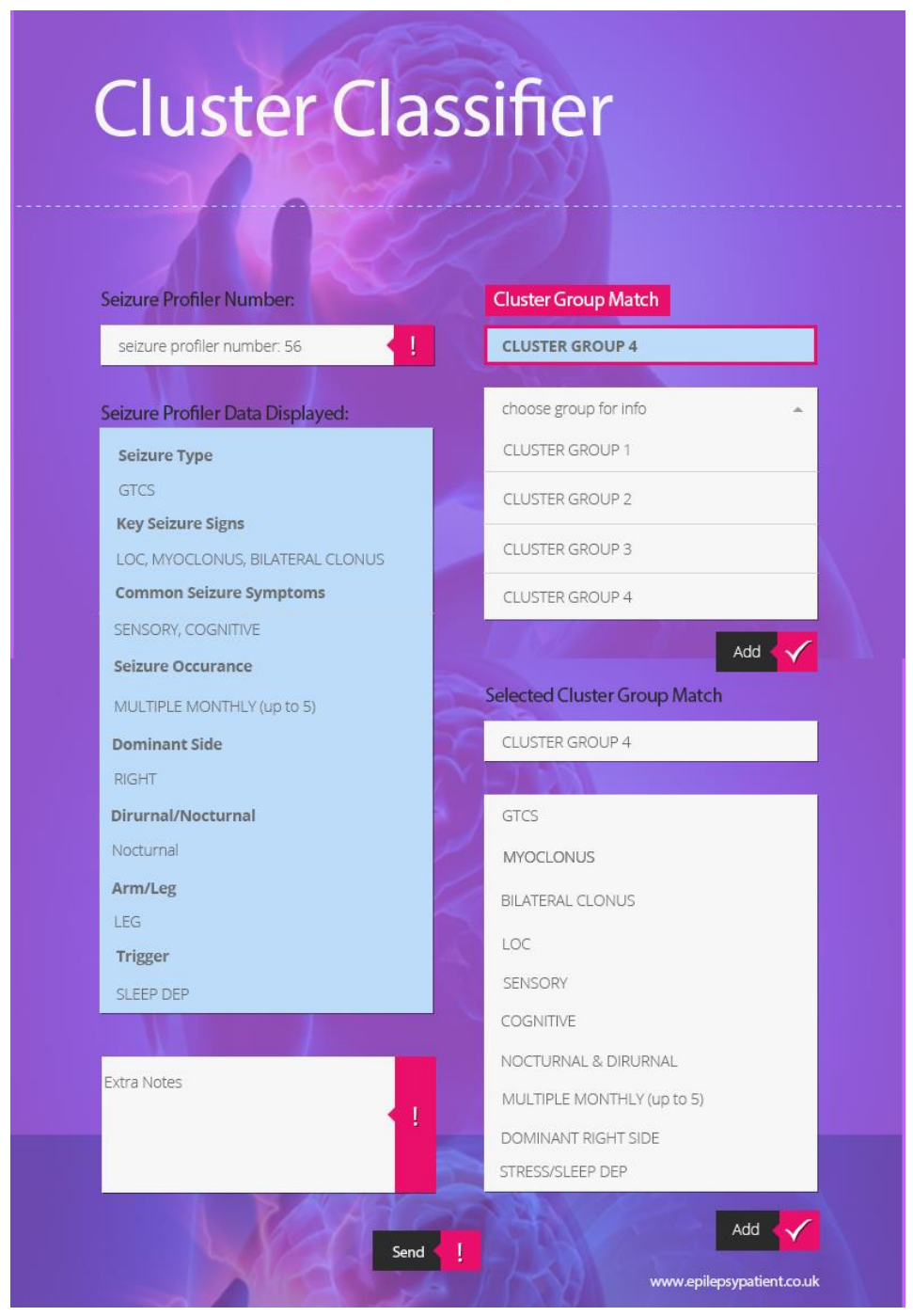

Figure 7.11. Cluster Classifier

4. The PMP framework passes the Cluster Classifier data to the 'Sensor Advisory' component. Here the HCP examines the Cluster Classifier data and checks that the recommended sensors and position are appropriate

The cluster group match for Patient Profile 56 (shown in Table.6.2) is 'Cluster Group 4'. For this Cluster Group the recommended sensor/sensors are the Accelerometer Sensor and Audio sensor to be positioned on the right leg. This is because this type of patient has a seizure symptoms which predominantly reveals itself on the right side of the body and begin in the leg. The patient is asked to wear the sensors at night-time (in bed) since they have nocturnal seizures. 
5. The PMP framework passes the three datasets to the 'Generate PMP' component: Seizure Profiler Data, Cluster Classifier Data and Sensor \& Position Information. These three datasets form the PMP for Patient Profile 56, see Figure. 7.12. PMP (Profile 56).

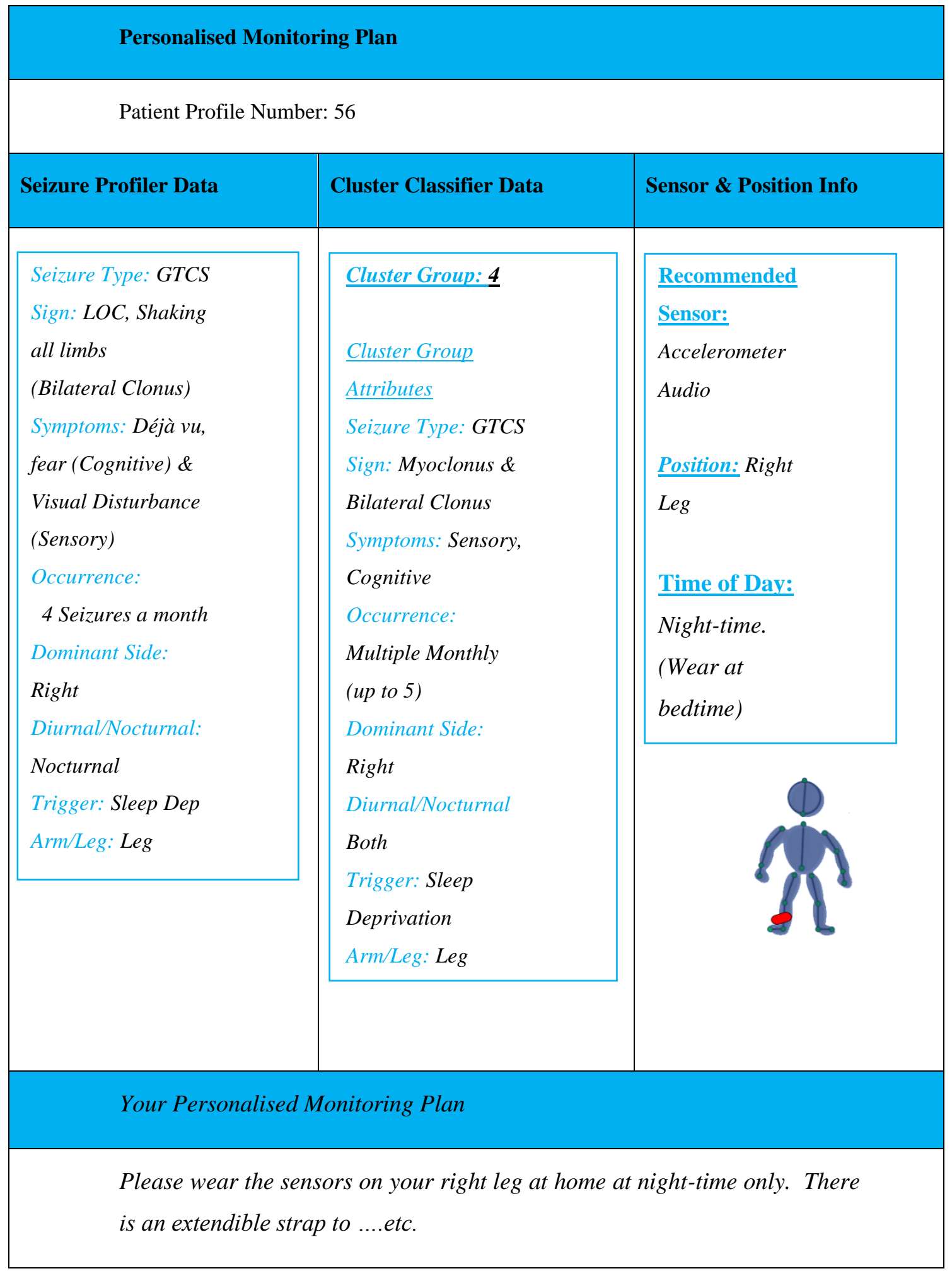

Figure. 7.12. PMP (Profile 56)

6. The patient receives their PMP 
7. The patient receives instructions and wears the IoT sensor-based device on the recommended body position. The patient completes a daily form and keeps a diary of the times of their known seizures.

8. The PMP frameworks sends the patients 'monitoring data' to the Health Service server.

9. The patient data is then analysed at the 'Hospital Analysis' stage of the PMP Framework using the 'Machine Learning Module' (as described in the 'Framework Components' section).

\subsubsection{Scenario 2}

Use Case Name: Scenario 2

Use Case Description: In this scenario an HCP takes a sample patient through each of the steps in the PMP framework

Actors: Patient Profile 57 (with an existing patient record)

Triggers: The patient indicates that he/she wants to have an IoT sensor-based device to remotely monitor their epilepsy.

Preconditions: The patient has known frequent seizures that have been recorded in the patient record.

\section{Post-conditions:}

i. The patient will receive a PMP (Personalised Monitoring Plan)

ii. The patient will receive a recommended IoT sensor-based device that they will place on position on their body.

iii. The patient's seizures will be monitored by the NHS.

\section{Normal Flow:}

1. The patient will indicate that he/she wants to have an IoT sensor-based device to remotely monitor their epilepsy and provides consent for the HCP to access their patient record.

2. The HCP using the PMP framework will explore the PPDL repository (see Step ' 2 ' in Figure 7.1. PMP Framework) to find possible feasible seizure types, signs and symptoms that have been observed by the HCP as 'key terms' in the patient record. See Figure 7.13. Patient Record Sample 57. 


\begin{tabular}{|l|l|}
\hline Hospital Number:\#\#\# & $\begin{array}{l}\text { Referring } \\
\text { Clinician:\#\# }\end{array}$ \\
\hline Test Type: EEG TELEM & Relevant Medical \\
\hline $\begin{array}{l}\text { CLINICAL HISTORY: } \\
\text { Age/date of onset- } 3 \text { years old. Description } \\
\text { of event: (Myoclonic tonic clonic), } 3 \text { in a } \\
\text { week, will occur when stressed after work, } \\
\text { during afternoons, can have full body- } \\
\text { jerking, on all sides of body but upper limbs } \\
\text { it begins, also tongue biting occurs. }\end{array}$ & Under review \\
\hline
\end{tabular}

Figure 7.13. Patient Record Sample 57

The patient record is personalised after a process of filtering upon entry to the 'Seizure Profiler' component (Figure 7.14. Seizure Profiler).

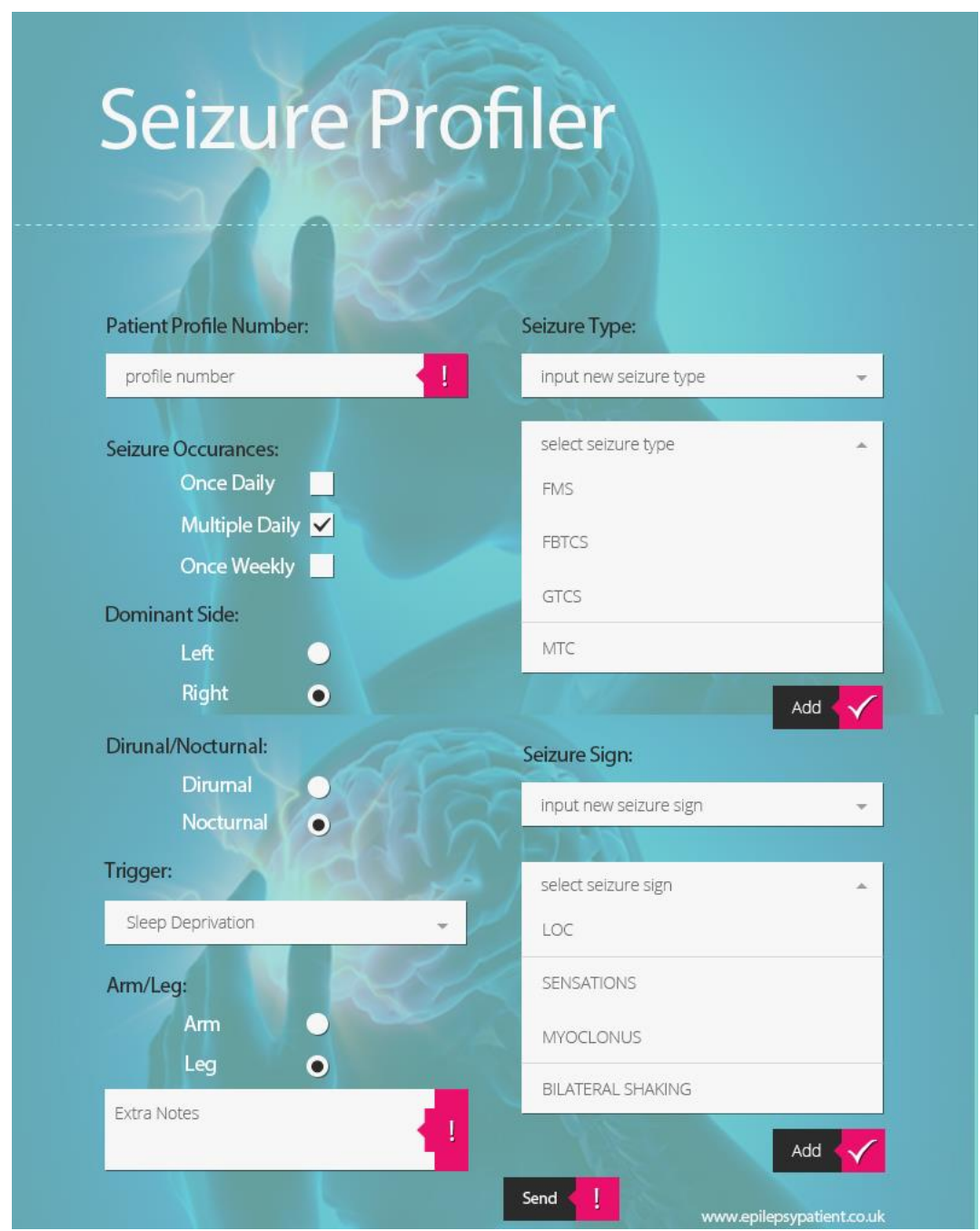

Figure 7.14. Seizure Profiler 
Automatic personalised seizure related data from the Seizure Profiler is fetched. The output is the Seizure Profiler Data. The patient output for Patient profile 57 is revealed here in Table 7.5. Keywords from the patient record have been incorporated and terminology updated.

Table 7.5. Seizure Profiler Data 57

\begin{tabular}{|l|l|l|l|l|}
\hline $\begin{array}{l}\text { Patient } \\
\text { Profile }\end{array}$ & $\begin{array}{l}\text { Seizure } \\
\text { Type }\end{array}$ & Key Signs & Symptom & Occurrence \\
\hline 57 & MTC & $\begin{array}{l}\text { Bilateral } \\
\text { Clonus }\end{array}$ & Automatisms & $\begin{array}{l}\text { Multiple } \\
\text { Weekly (up } \\
\text { to 5) }\end{array}$ \\
\hline $\begin{array}{l}\text { Dominant } \\
\text { Side }\end{array}$ & Diurnal/Nocturnal & Trigger & Arm/Leg \\
\hline Either & Diurnal & Stress & Arms \\
\hline
\end{tabular}

3. The PMP framework passes the 'Seizure Profiler Data' to the 'Cluster Classifier' component. The PMP framework matches the 'key terms' from the 'Seizure Related Profile Data' to the correct 'Cluster Group' i.e. the sample in 'Patient profile 57' is matched to 'Cluster Group 5', see Figure 7.15. Cluster Classifier Profile 57 


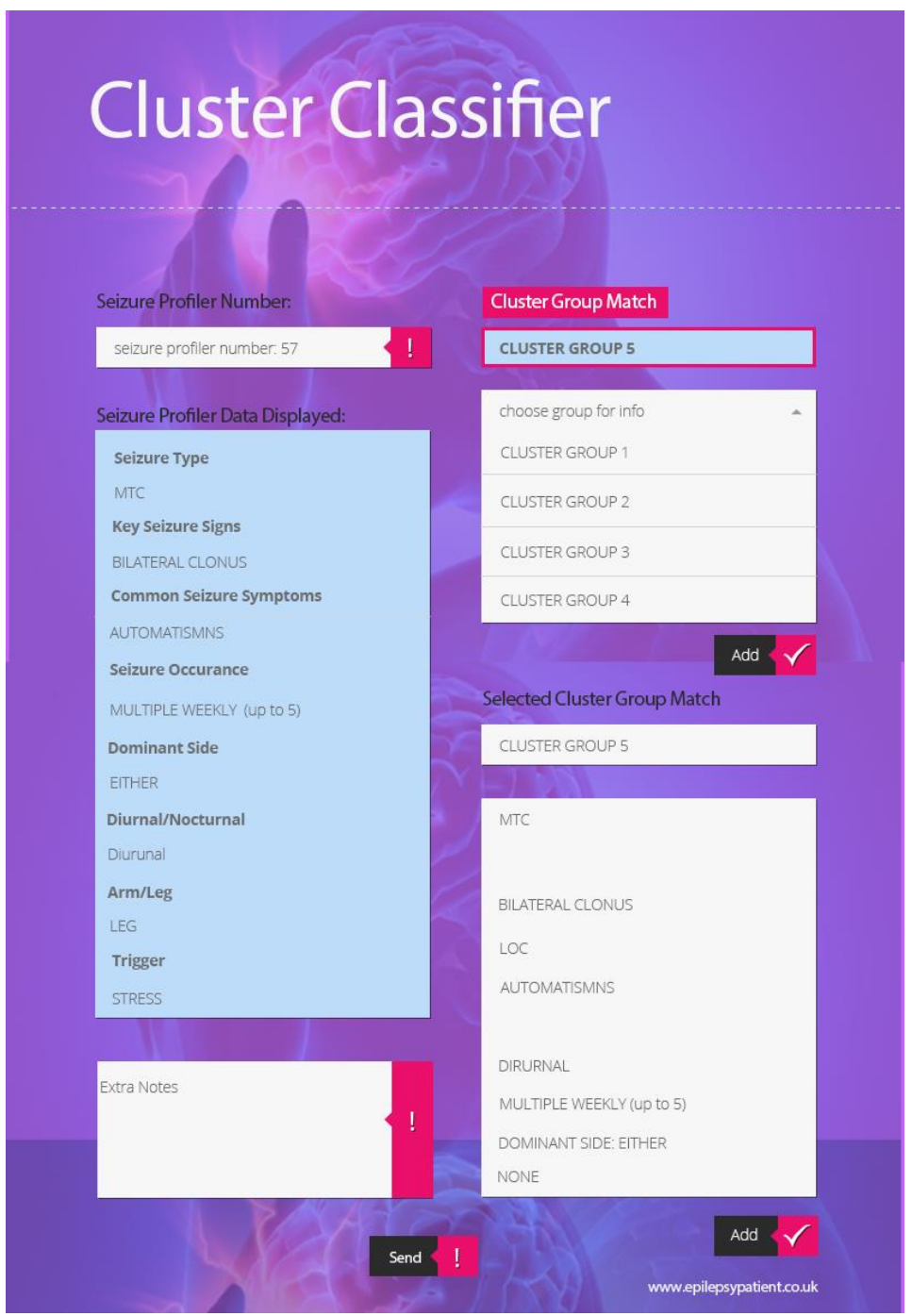

Figure 7.15. Cluster Classifier Profile 57

4. The PMP framework passes the Cluster Classifier data to the 'Sensor Advisory' component. The HCP examines the Cluster Classifier data and checks that the recommended sensors and position are appropriate.

The cluster group match for Patient Profile 57 shown in Table.6.2 is 'Cluster Group 5.' For this Cluster Group the recommended sensor/sensors are the Accelerometer Sensor and Heartrate sensor to be positioned on the right wrist. This is because this type of patient has a seizure type which reveals itself on both sides of the body and begins in the upper limbs. As the patient has Diurnal seizures, they are asked to wear the sensors during the daytime only.

5. The PMP framework passes the three datasets to the 'Generate PMP' component: Seizure Profiler Data, Cluster Classifier Data and Sensor \& Position Information. These three datasets form the PMP for Patient Profile 57, see Figure 7.16. PMP Sample 57 


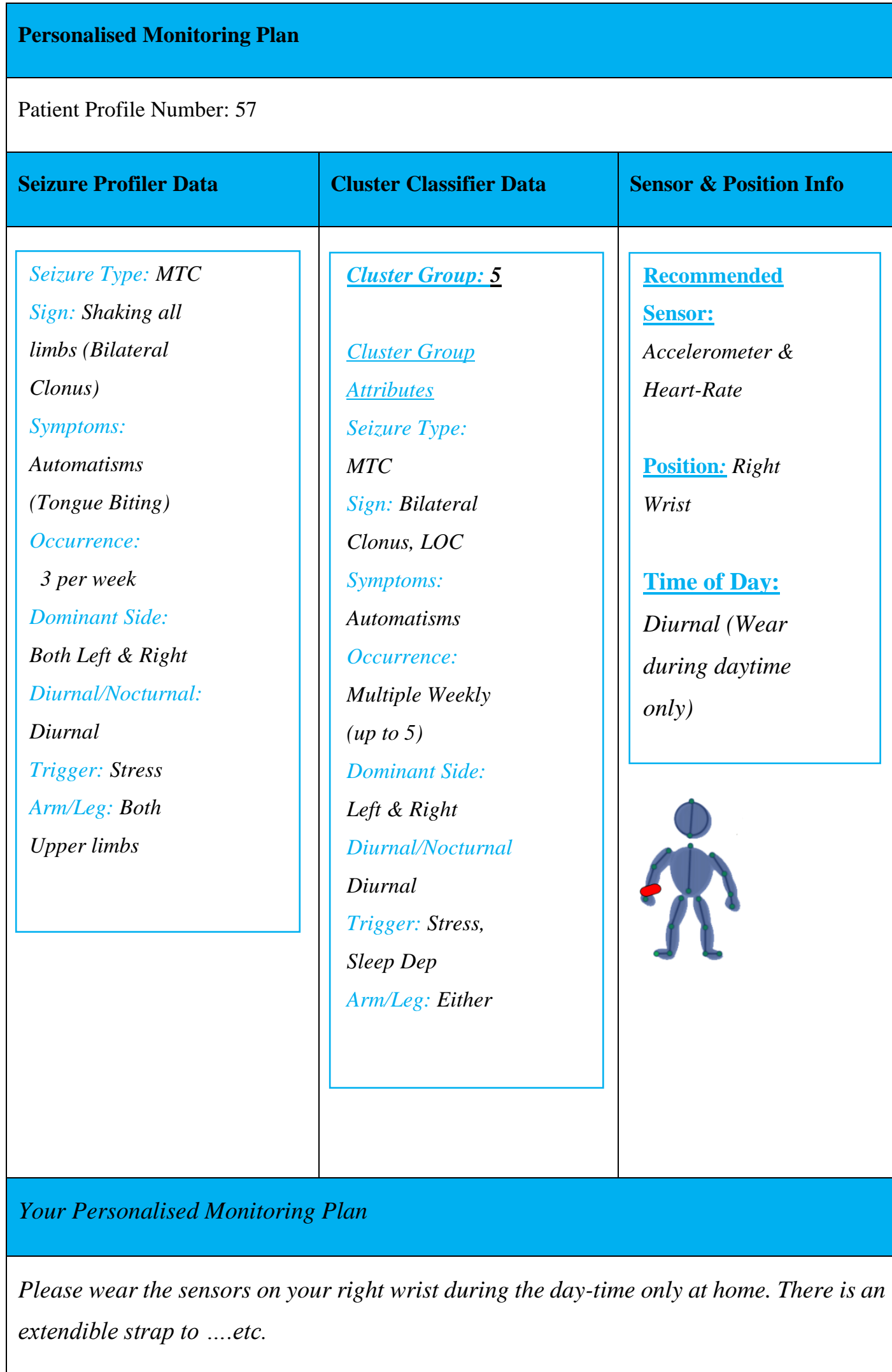

Figure 7.16. PMP Sample 57

6. The patient receives their PMP

7. The patient receives instructions and wears the IoT sensor-based device on the recommended body position. The patient completes a daily form and keeps a diary of the times of their known seizures. 
8. The PMP frameworks sends the patients 'monitoring data' to the NHS server.

9. The patient data is then analysed at the 'Hospital Analysis' stage of the PMP Framework using the 'Machine Learning Module' (as described in the 'Framework Components' section).

\subsection{Proposed IoT for Epilepsy Paradigm}

To achieve the type of monitoring described in the PMP framework, several IoT components can be deployed to retrieve sensor data from the epilepsy patient to be accessed remotely. These components include the integration of the personalisation components described in the PMP framework, those of an internet connection and protocols which form the 'network layer', a cloud platform to manage the data analysis and fundamentally the sensor-based devices forming the sensor layer. These components make the ingredients of an IoT solution, proposed in the IoT Epilepsy Paradigm.

The purpose of the IoT Epilepsy Paradigm is to support the PMP framework in collecting data from a variety of potential epilepsy device sensors and also provide optimal analysis tools to utilise the sensor data thus supporting clinicians to monitor epilepsy patients.

\subsubsection{Sensor Layer}

The sensor layer has the task of acquiring and sending the data from the different epilepsy devices involved in capturing seizure data, to the proposed cloud platform.

The sensors previously used in the experiment, the heart-rate and accelerometer sensors were demonstrated within the PMP framework discussed in this chapter. Yet there are other sensors too that can work within the context of this research. These potential sensors found in other devices are explored and proposed below.

\subsubsection{Sensors for Epilepsy}

Despite the expense, multi-modal sensor based devices are the ultimate desire to monitor an epilepsy patients seizures since multiple sensors are embedded in one device and make comfortability for the patient and all-in-one solutions for the manager of the device, furthermore epilepsy patients have revealed their preference for devices capable of monitoring several parameters [229]. Section 2.3 in Chapter 2 uncovered some of the epilepsy detection devices and monitoring systems, whilst Chapter 6 demonstrated the use of an inexpensive device with heart-rate and accelerometer sensor (justified for experimental purposes), and simultaneously identified other useful sensors for epilepsy. These other sensors which exist in devices, those that go beyond heart and movement sensing, and beyond fitness devices, are amongst a vast amount. Consequently, many studies have analysed the 
performance and limitations of each sensor based device, one critical evaluation by Peake [230] found many devices where not yet fully validated or tested for reliability, therefore this examination will aim only to propose validated devices for use in the IoT Epilepsy paradigm.

Recently in 2020, Abreu, Fred et al [231] did a significant exploration on, wearables and related devices, that can be utilised for epilepsy prediction, the findings are produced in tables shown in Table 7.7 Wearable Sensor Exploration [231] which presents devices, some with multiple sensors, characterised with respect to their applicability to research, validation status, form factor or body positioning, battery duration, method to access the data, measured signals and, their applicability to epilepsy prediction (EP) [231].

This is a useful table since the devices have already been validated, and connectivity options identified, and since they are beneficial for epilepsy they can be proposed in the IoT Epilepsy Paradigm.

These devices and their sensors, identified as applicable for epilepsy prediction contain some of the sensors that were observed in the sensor advisory section (7.4.1) and shown Figure 7.7, i.e. accelerometer, heart-rate and GSR, but the audio and bladder sensors are not included. In this case the audio and bladder are added to Table 7.6 Selected Sensors for IoT Epilepsy Paradigm, together with their connectivity options.

The 'IoT Epilepsy Paradigm' proposal in Figure 7.17 shows areas on the body where parameters are measured, each area is indicated with a colour matching the parameter. The chosen devices have been proposed based upon the factors in Table 7.7 Wearable Sensor Exploration [231] for the best battery life, validity, and connectivity options selected for ease of connection to the cloud platform in the IoT Epilepsy Paradigm. Since Embrace2 device uses its own onboard processing it is not adaptable for the paradigm proposed in this study. Furthermore some of the device based sensors depicted in Table 7.7 Wearable Sensor Exploration [231] are multi-modal so their use can sometimes be proposed in more than one activity area, for example the device EQ02 has both the heart-rate and temperature sensor. 
Table 7.6 Selected Sensors for IoT Epilepsy Paradigm

\begin{tabular}{|l|l|l|l|l|}
\hline Body Area & $\begin{array}{l}\text { Validated } \\
\text { Device }\end{array}$ & Sensor & $\begin{array}{l}\text { Connectivity } \\
\text { Option }\end{array}$ & $\begin{array}{l}\text { Battery } \\
\text { Life }\end{array}$ \\
\hline Breathing & HexoSkin & RESP & $\begin{array}{l}\text { Cloud Storage } \\
\text { or BLE } \\
\text { transmission }\end{array}$ & $12 \mathrm{~h}$ \\
\hline Electrodermal & Empatica E4 & $\begin{array}{l}\text { EDA } \\
\text { or GVS }\end{array}$ & Cloud Storage & $48 \mathrm{~h}$ \\
\hline Blood Volume & Empatica E4 & PPG & Cloud Storage & $48 \mathrm{~h}$ \\
\hline Movement & Bioharness3 & ACC & $\begin{array}{l}\text { BLE } \\
\text { transmission }\end{array}$ & $12-24 \mathrm{~h}$ \\
\hline Heart-Rate & EQ02 & ECG & $\begin{array}{l}\text { Bluetooth } \\
\text { transmission }\end{array}$ & $48 \mathrm{~h}$ \\
\hline Temperature & EQ02 & Temp & $\begin{array}{l}\text { Bluetooth } \\
\text { transmission }\end{array}$ & $48 \mathrm{~h}$ \\
\hline Audio & Alert-it & $\begin{array}{l}\text { Sound } \\
\text { Sensor }\end{array}$ & $\begin{array}{l}\text { Ethernet } \\
\text { connection }\end{array}$ & $\begin{array}{l}\text { Bluetooth } \\
\text { transmission }\end{array}$ \\
\hline $\begin{array}{l}\text { Bladder/ } \\
\text { Incontinence }\end{array}$ & DFree & Ultra- & $24 \mathrm{~h}$ \\
\hline
\end{tabular}


Table 7.7 Wearable Sensor Exploration [231]

\begin{tabular}{|c|c|c|c|c|c|c|c|}
\hline Product & Research use & Validated/ & Site & Battery & Data Accessibility & Sensor & Suitability EP \\
\hline EQ02 [55] & Ambulatory [56], Athletes & Validation with Holter & Vest & $48 \mathrm{~h}$ & Bluetooth transmission & ECG, Derived RESP, & $\sqrt{ }$ \\
\hline E4 [59] & Migraines [60], Stress Detection & CE clearance [12] & Bracelet & $+48 \mathrm{~h}$ & Cloud Storage & EDA, PPG, Temp & $\sqrt{ }$ \\
\hline BITalino [62] & Biometrics [63], Parkinson's [64] & Validation with BioPac & Non & $12-24 \mathrm{~h}$ & Bluetooth transmission & ECG, EDA, EMG, BVP, & $\sqrt{ }$ \\
\hline VisiMobile & Ambulatory Monitoring [67] & FDA ${ }^{\text {a clearance }}$ & Wristband & $+12 \mathrm{~h}$ & WiFi transmission & ECG, RESP, SpO2, & $\sqrt{ }$ \\
\hline Bioharness3 & Sports Monitoring & HR validation [69] & Chest & $12-24 \mathrm{~h}$ & BLE transmission & ECG, RESP, Temp, ACC & $\sqrt{ }$ \\
\hline Hexoskin [70] & Remote Monitoring & Experimental Validation & Smart & $12 \mathrm{~h}$ & Cloud Storage or BLE & ECG, RESP, ACC & $\sqrt{ }$ \\
\hline Vivosmart4 & Stress Assessment & Not yet validated & Smartwatc & 5 days & BLE transmission & Optical HRV, SpO2, Light, & $\mathbf{x}$ \\
\hline
\end{tabular}

\begin{tabular}{|c|c|c|c|c|c|c|c|}
\hline Product & Research use & Validated/ Reliable & Site & Battery & Data Accessibility & Sensors & Suitability EP \\
\hline Moodmetric & Stress Assessment [56] & Experimental comparison & Ring & 4 days & BLE Transmission & EDA & $\mathrm{x}$ \\
\hline Embrace2 [59] & Seizure Detection & FDA and CE clearance & Smartwatch & $+48 \mathrm{~h}$ & Onboard Processing & EDA, Temp, Acc, & $\sqrt{ }$ \\
\hline GoBe2 [87] & Nutrition, Hydration, & Only for calorie intake [88] & Smartwatch & $24 \mathrm{~h}$ & BLE transmission & EDA, Acc, Gyr, Mag & $\sqrt{ }$ \\
\hline MyFeel [89] & Emotion & Preliminary study & Wristband & $+24 \mathrm{~h}$ & BLE Transmission & EDA, Temp & $\mathrm{x}$ \\
\hline Pip [90] & Stress Assessment & Not yet validated & External & $+8 \mathrm{~h}$ & Cloud Storage and BLE & EDA & $\mathrm{x}$ \\
\hline
\end{tabular}




\subsubsection{Cloud Platform}

The proposed cloud platform provides all the necessary services for the clinician to manage, process and visualise the seizure data. All the processes that involve the interaction between the personalisation layer and the sensor layer are carried out through the following modules: PMP data management, machine learning module and data analysis \& visualisation. All these services are hosted in the cloud and clinicians are able to access them remotely from any location. The data analysis and visualisation module utilises the sensor data while the 'PMP data management' module pulls all the patient records from the personalisation modules and here the sensor data results are updated. Visualisation is a requirement for any such system as it is important for clinicians to be provided with user friendly GUIs so they can study the seizure data from the epilepsy sensor devices. The machine learning module is also proposed, this is a key aspect for future development and the idea is that by using algorithms the module will 'learn' when a patient is about to have seizure and warn them in advance.

A pre-processing hardware and a platform are needed to communicate and transmit the sensor data which is collected using wearable sensors positioned on a patients body. The Microsoft Azure IoT platform [232] is proposed, since this cloud computing server is trusted and safe [233].

\subsubsection{Network Layer}

There are several ways the sensors can connect and send data to the cloud platform and since most of the sensor devices connect to a mobile phone they are served by Bluetooth or Bluetooth Low Energy (BLE) and use very little power. Nevertheless each sensor based device is provided with its own protocol and connectivity options as noted in Table 7.6 hence the type of IoT connectivity is determined generally by the distance that the data must travel, either short-range or long-range [234]. IoT platforms such as Azure use gateways to connect IoT devices to the cloud. The data collected from the devices moves through this gateway, gets pre-processed using in build modules (Edge) and then gets sent to the cloud. Data is protected by an additional layer of security provided by the Azure Application gateway and in addition connection security is enabled as each connected IoT device is given a unique identity key [234].

\subsubsection{Scope of IoT Epilepsy Paradigm}

An IoT approach has been proposed, this IoT epilepsy paradigm can be adopted by the PMP framework and has demonstrated some of the validated sensors for epilepsy monitoring. It is outside the scope of this thesis to develop and perform monitoring upon each of the sensor based devices shown in the IoT Epilepsy Paradigm, and it should be noted that there are many more considerations to be taken into account for an IoT 'solution', one such example is to deploy an additional component to closely monitor the function of a sensor and send an alert to the user if a sensor were to malfunction. 


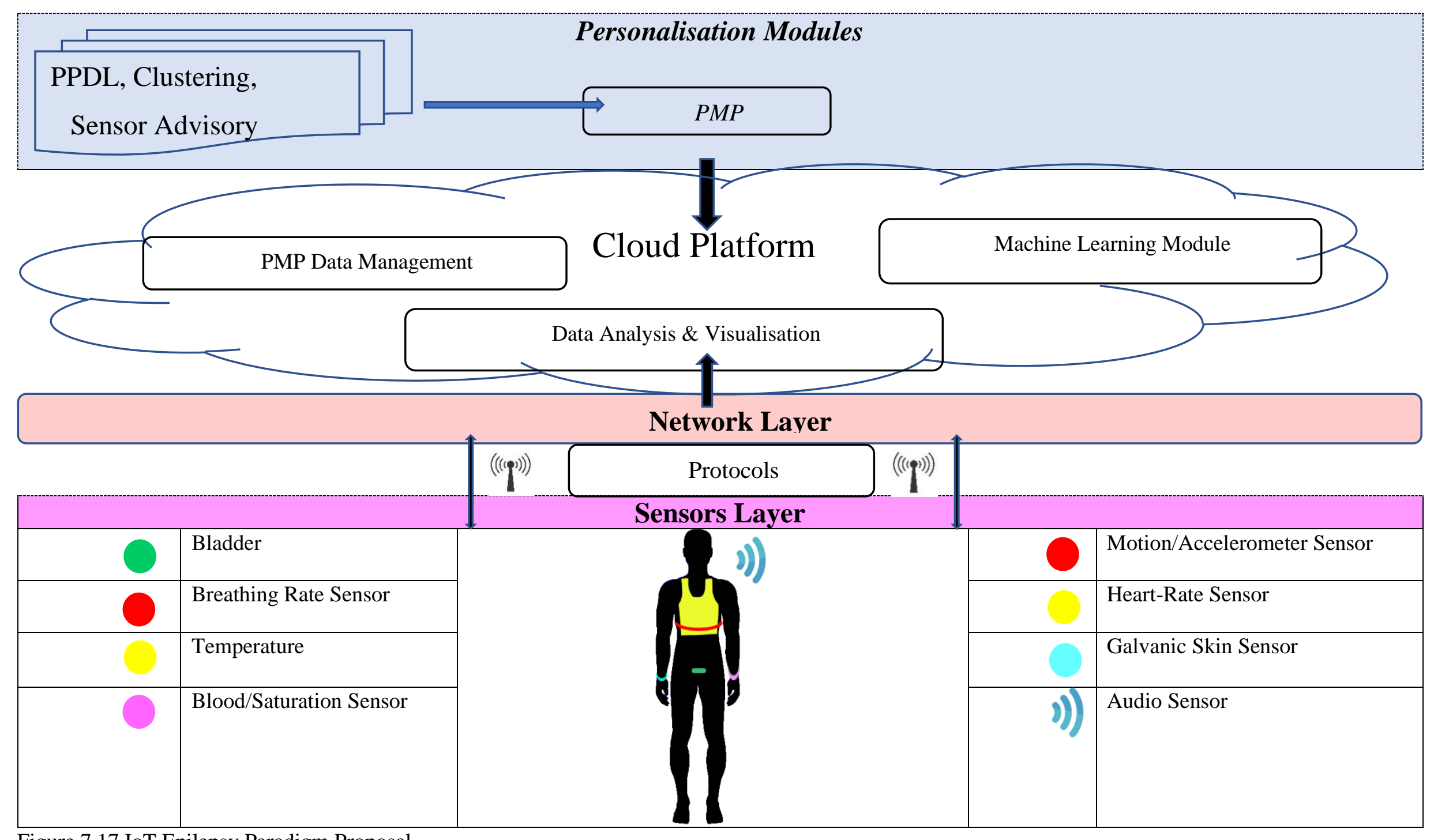

Figure 7.17 IoT Epilepsy Paradigm Proposal 


\subsection{Summary}

The integration of the components and technologies in the framework depicted in Figure 7.1. PMP Framework aims at providing HCP's dealing with epilepsy patients with an integrated tool that helps them in recommending the correct IoT sensor and position on the patient's body.

These decisions are made at the point and time of care as an 'aid' in personalising the condition of new arriving patients, and to refine the predefined 'patient record' in order to obtain and validate a Personalised Monitoring Plan which is in addition adapted to include the seizure monitoring of the patient during appointments.

The PMP Framework is designed to provide a patient-empowering support in a way that the available knowledge is continuously personalised to the condition of the seizure type patient. The IoT Epilepsy paradigm has been proposed and can be adopted by the PMP framework in future developments. 


\section{Chapter 8 Conclusion and Future Work}

\subsection{Introduction}

This final chapter seeks to conclude the thesis with a summary of its goals and contributions, a discussion of barriers and limitations, followed by proposed improvements and directions for future work.

\subsection{Contributions and Achievements}

The main contribution of this research set out to develop an IoT driven framework for remote personalised monitoring and managing of epilepsy to help HCP's to create a 'Personalised Monitoring Plan'. The research began by exploring the challenges and issues surrounding epilepsy and healthcare monitoring systems proposed by researchers and technologies to help 'detect' seizures. The concept of Personalised Healthcare was introduced, and methods were observed to make epilepsy patients distinct from one another. It was discovered here how IoT can be utilised to support the ever growing trend of Personalised Healthcare but during this literature review it was found that currently, epilepsy monitoring systems and IoT platforms do not fully embrace a personalised approach, despite some observations in studies about the importance of distinguishing each 'seizure type'. Since 'seizure type' is just one of many parameters that can distinguish one seizure patient from another, this thesis set out to identify and investigate any more very individual characteristics to address the challenge to achieve a truly 'personalised' approach to managing epilepsy.

In addition to fulfilling the aim of this thesis the following contributions were made:

A.) Epilepsy 'terminology' was first investigated; existing seizure type classifications/categories were analysed so that an individual seizure type patient profile could be formed and thus an ontology was developed to model this concept, namely ESO 'Epilepsy Seizure Ontology'. This was a driving force for the PMP Framework and a critical aspect for this concept. In order to make ESO useable for HCPs (Health Care Professionals) the ontology was transformed into a language that is understandable by humans and machines, this was accomplished by XML and the outcome was PPDL (Patient Profile Description Language).

B.) To see if distinct group of patients sharing similar characteristics could be identified so that ultimately patients could receive distinct recommendations per group Clustering techniques were analysed and carried out. The outcome was a set of 6 distinct 'clustering' groups, these 6 cluster groups revealed six completely different categories of patients each 
with their distinct seizure related information. This outcome is extendable: the use of a single dataset means that the results should not be generalised to a wider population. For future work, multiple datasets should be used for further validation.

C.) To test the assumption to see if an individual patient profile or distinct group of patients sharing similar characteristics can make a difference in which IoT device to use experiments were performed to capture seizure data, obtained from sensors, which were positioned on different parts of the patient's body. The experiment outcomes revealed that difference is observed due to sensor 'position' on the body and that sensor 'types' are suited to different patient profiles. Therefore, these results were used to inform the development of the conceptual PMP Framework which was developed to evaluate the research.

D.) The research design approach used in this thesis shown in Chapter 3 Figure 3.1 originated on findings from the literature review. However, this framework is a new and novel way to achieve the type of 'personalisation' described in this thesis. This overall design approach which incorporates a sequential strategy is transferrable to other studies aiming to achieve personalisation within alternative domains or can be applied to other healthcare conditions such as diabetes, asthma or a condition with many characteristics.

The principal contribution in this thesis was that with the prior 'knowledge' of individual patient characteristics drawn from the PPDL repository and 'Cluster Groups' together with the supplementary 'proof of concept' knowledge obtained in the experiments each epilepsy patient can be treated distinctly and recommended an appropriate sensor-based device thus forming a patient specific unique PMP (Personalised Monitoring Plan). Hence personalisation can be achieved.

\subsubsection{Barriers and Limitations}

In order to carry out testing upon patients in real hospital settings a full 'Integrated Research Approval System' (IRAS) ethics application was carried out. The application was successful, yet it was demanding, time-consuming and vigorous. It included verbal interviews and seeking consent from various NHS bodies in addition to the 'actual writing' which required the author to understand new medical terminology. Despite this large-scale application and the proposed 20 patient recruitment, only 6 were recruited after all.

Prior to creating this novel PMP Framework IoT sensor-based devices and their capabilities for epilepsy management where investigated. Finding a suitable IoT sensor-based device was challenging as there were many caveats to consider, such as finding just the one device which contained the appropriate sensors, affordability and most importantly that the device would allow data extraction in a raw form, so that the data could be analysed. 
Some technical failure occurred with HP4 because the patient was either not wearing the watch at the time of the seizure or neither the sensor-based device nor the online server captured the event, as the records for 2 seizure event times were skipped during data transfer. Similarly, with the excluded patient, heart-rate data was recorded, however, the server although detecting the date and time of the seizure did not record any accelerometer data.

All participants wearing the sensor-based device had sufficient battery power over the 5-day period, although clinicians reported that battery power drained quickly after this period. The battery life of sensor-based devices is a common problem and it is essential that the wearable device is powered by a reliable battery to measure everyday health indicators or specifically if they are used in a life-critical device [235]. There is currently work being done to use thin cell batteries to tackle this problem [235].

\subsection{Recommendations and Future Work}

During the development of this thesis several areas have highlighted potential for future direction of this research, these proposals are discussed below.

\subsubsection{PPDL (Patient Profile Development Language) and Clustering}

The PPDL in its current form contains appropriate knowledge to answer the objectives. However, concepts presented in the PPDL and used in the clustering process can evolve in the future expanding the knowledge base. This aspect has already been catered for in the conceptual PMP framework design i.e. As patient's present new signs and symptoms these classes can be added by the HCP, in order to maintain the real-world adoption of the PPDL. It is also worth mentioning that a universal acceptance of the new terminology in the ILAE should also be adhered to so that concepts such as PPDL and the 'Cluster Groups' proposed in this study can be more widely used.

It is recommended in the future that further time can be spent on clustering. The next stage can trial some of the other available clustering techniques explored in Chapter 5. If the clustering technique K-means used to achieve the cluster groups in this study were to be used in the future there is also scope for deeper analysis upon 'choosing the k'.

\subsubsection{Machine Learning and Sensor Decision Tree}

One of the most significant future directions for long-term remote monitoring of epilepsy is seizure detection via 'machine learning'. By accumulating large datasets, computers can learn by recognising patterns in data. This automated approach (without human intervention) has been proposed as a 'machine learning module' within the PMP framework and IoT Epilepsy 
Paradigm to determine seizure detection or not based on the patient specific profile, the idea being that by using algorithms the module will 'learn' when a patient is about to have seizure and warn them in advance. Due to limitations in this study this module has not been built but instead is proposed at this stage and recommended for the next level. Largely there is further work to take the PMP conceptual model and the IoT Epilepsy Paradigm (discussed below) into full operation.

By using the philosophy of machine learning once again, it is a future recommendation to advance the sensor decision tree, (Figure 7.7. Sensor Decision Tree) shown in Chapter 7. This future advancement was already discussed and highlighted that a future goal is to use the decision tree as a training model that can be used to predict the target variable, i.e. the sensor, by learning simple decision rules inferred from prior training data. It is recommended that in future data exploration more variables will be added such as signs/symptoms, sensors and positions, and therefore the decision tree can help identify the most significant variable. This way the sensor decision tree can grow so that the outcome can be predicted, whereby in each leaf node the average outcome of the training data in this node is used. Similarly, this approach can be adopted by other health domain experts in other areas such as stress management, asthma or where multi sensors can treat different symptoms.

\subsubsection{IoT Epilepsy Paradigm and Robustness}

Section 7.7.4 in Chapter 7 identified that it is outside the scope of this thesis to develop and perform monitoring upon each of the sensor-based devices shown in the IoT Epilepsy Paradigm. This thesis demonstrated an 'approach' and presented an IoT 'model' only.

It is recommended that the IoT Epilepsy paradigm be taken to the next level in future research. For the adoption of cloud computing the Microsoft Azure was proposed, yet the choice of a number of ready built elements that enable hardware manufacturers to connect their devices to the internet are vast and as such there were no less than ' 450 organisations advertising' an "IoT Platform" in 2017 [236]. The tools required for IoT solutions offer everything from open source software SDKs and architecture design patterns to cloud-based IoT APIs and soldered hardware chipsets [236].

It is expected the number of sensor-based devices connected to the IoT Epilepsy Paradigm will grow over time. For the infrastructure to be effective it must be robust and that can only be enabled through thorough testing [237]. Chapter 7 recommended deploying an additional component to closely monitor the function of a sensor and send an alert to the user if a sensor were to malfunction, this is one of many scenarios that must be tested and it is recommended that simulating these scenarios during performance testing should be arranged in the future to aid building a robust IoT system. 


\subsubsection{Embrace for Research platform}

During the experiments in Chapter 6 data retrieval using the light-weight Fitbit sensor-based device proved easy to use with adequate battery life appropriate for this research however other alternatives in the future should be pursued.

One such dependable method is the 'Embrace for Research' platform, by Empatica, see Figure 8.1 below [238]. Not only are there more sensors contained in the wearable device, the data retrieval process is much smoother hence data-flow is seamless via a Bluetooth connection to the Mate app then the raw data is transmitted continuously to the Research Portal where researchers can review at a glance [238]. This platform was too expensive to use in the experiments in this study and during enquiries (at the time of experiments) it was alleged there were no such 'extendible' straps available to allow the device to be positioned on different parts of the body as opposed to the wrist, this being an area of potential future direction.

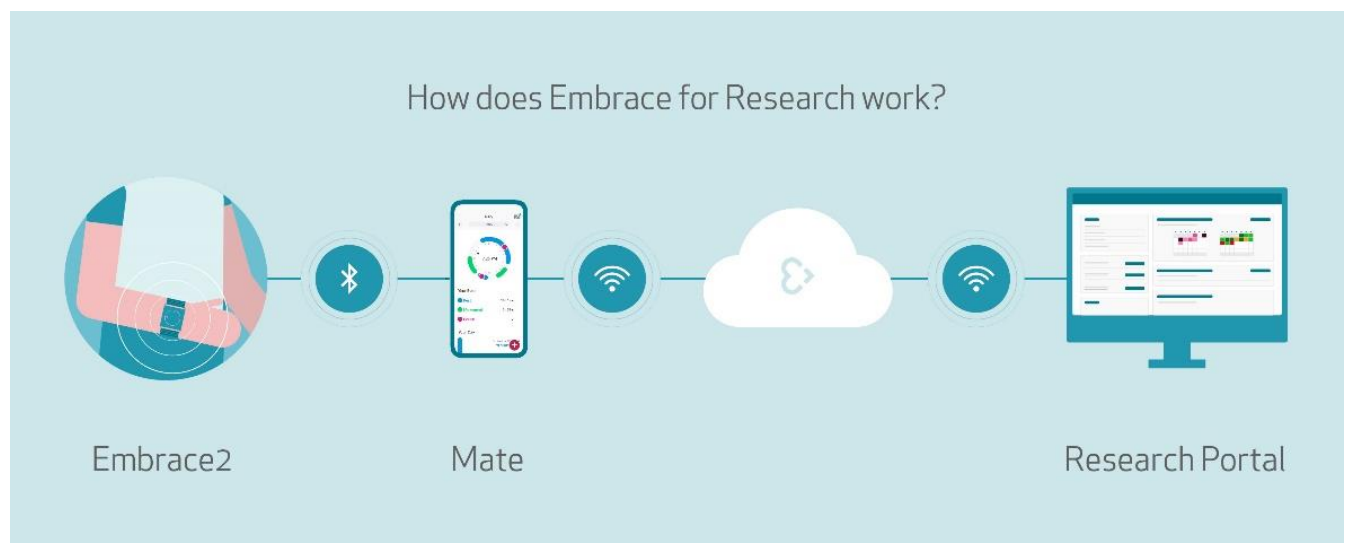

Figure 8.1. Embrace for Research Platform [238]

\subsubsection{Long Term Uses \& Applicability in other domains for the PMP Framework}

The methods used in this thesis for ontology development and clustering analysis can be applied to any disease whereby recognised symptoms per patient can be individualised and be further put into sub-groups or categories. However, to fully utilise this personalised approach the application of the PMP framework can be particularly applied to patients whom have symptoms that can be monitored with different IoT sensor-based devices and personalised further by wearing the device on different body positions. In the future the following types of patients can be handled by the proposed PMP framework: (shown below together with latest progressive recommended sensor-based devices).

- Diabetes: i.e. One such recommended device could be use of 'flash glucose sensing': A device which checks blood glucose levels by scanning a sensor worn on their arm will be (available on the NHS for people with type 1 diabetes) [239]. 
- Sick Infants: i.e. A recommended device could be use of a miniaturised, wireless oxygen sensor wearable device the size of a Band-Aid which would allow babies to be monitored from home and able to leave the hospital [240].

- Rehabilitation: i.e. The recommended device for rehabilitation could be a Force-based sensor which can be integrated with footwear to measure the interaction of the body with the ground during walking [241]. Due to the possibility of detecting not only physiological but also movement data wearable sensors have also acquired increasing importance in the field of rehabilitation [212].

\subsection{Retrospection}

If this research problem were to be taken now would a different approach be adopted? This requires a thoughtful answer. The first thoughts are driven instinctively to the research questions i.e. can the remote monitoring of individual patients be personalised using an IoT approach? In truth, yes, the research questions have been answered fittingly and the problem solved.

Looking back, the approaches taken to achieve the answers were rather combined for example by incorporating ontology development, clustering analysis and IoT technology. Therefore, if the thesis had focused 'solely' on using one approach i.e. clustering techniques, it stands to reason more time would have been dedicated to it, would there have been a different/stronger outcome? There would have been more experiments with other clustering algorithms aside from K-means and more exploration in how to 'choose the $\mathrm{K}$ ' but there is no certainty the cluster group outcomes would have been more accurate or looked any different, besides which the concept of clustering epilepsy patient profiles is clearly demonstrated and the underpinning work in place to develop this further. Moreover, without the depth of the ontology development approach the dataset created from the ESO which uncovered a variety of patient profiles, the clustering outcome would not have been so vigorous. Similarly, looking back, the IoT approach served to furnish the remote personalised monitoring of individual patients by highlighting the essential IoT sensor-based devices that can monitor epilepsy. The IoT approach built upon the contributions from the ontology and clustering approaches, capturing without human interaction how the typical seizure patient profiles can be represented.

Aside from wishing that patient recruitment had begun earlier, being too ambitious about the amount of epilepsy patients that could be recruited for experiments, or in hindsight pursuing more funding to be able to afford 'out of the box' equipment it can be said that within the scope of this thesis there would not have been much done differently. 
The premises made in this thesis put the future of personalisation in epilepsy into focus, a focus that shifts from a one-size fits all to a focus on the core of the epilepsy patients' individual characteristics. The computer science contributions made are a ' $\operatorname{cog}$ in the wheel' in this arena, and these stepping stones hope to pave the way for a brighter future in epilepsy monitoring and management. 


\section{References}

[1] P. M. Vergara, E. de La Cal, J. R. Villar, V. M. González, and J. Sedano, “An IoT Platform for Epilepsy Monitoring and Supervising," Journal of Sensors, vol. 2017, 2017, doi: 10.1155/2017/6043069.

[2] D. Callegari et al., "EpiCare - A home care platform based on mobile cloud computing to assist epilepsy diagnosis," in Proceedings of the 2014 4th International Conference on Wireless Mobile Communication and Healthcare - "Transforming Healthcare Through Innovations in Mobile and Wireless Technologies", MOBIHEALTH 2014, 2015, pp. 148-151, doi: 10.1109/MOBIHEALTH.2014.7015931.

[3] A. Ulate-Campos, F. Coughlin, M. Gaínza-Lein, I. S. Fernández, P. L. Pearl, and T. Loddenkemper, "Automated seizure detection systems and their effectiveness for each type of seizure," Seizure, vol. 40. W.B. Saunders Ltd, pp. 88-101, 01-Aug-2016, doi: 10.1016/j.seizure.2016.06.008.

[4] "Wearable Devices Aim to Monitor Epileptic Seizures." [Online]. Available: https://www.webmd.com/epilepsy/news/20151208/wearabledevices-aim-to-monitor-epileptic-seizures\#1. [Accessed: 05-Feb-2020].

[5] "Facts and statistics | Epilepsy Society." [Online]. Available: https://www.epilepsysociety.org.uk/facts-and-statistics\#.XeEoAOj7TIU. [Accessed: 29-Nov-2019].

[6] "What Is Epilepsy? | Live Science." [Online]. Available: https://www.livescience.com/34723-epilepsy-symptoms-andtreatment.html. [Accessed: 05-Feb-2020].

[7] N. Moghim and D. W. Corne, "Predicting Epileptic Seizures in Advance," PLoS ONE, vol. 9, no. 6, p. e99334, Jun. 2014, doi: 10.1371/journal.pone.0099334.

[8] Bernard S. Chang and Daniel H. Lowenstein., "Epilepsy," The New England Journal of Medicine, vol. 349(13), pp. 1257-1266, 2003.

[9] "Epilepsy facts and terminology | Epilepsy Action." [Online]. Available: https://www.epilepsy.org.uk/press/facts. [Accessed: 30-Nov-2019].

[10] L. Chen et al., "OMDP: An ontology-based model for diagnosis and treatment of diabetes patients in remote healthcare systems," International Journal of Distributed Sensor Networks, vol. 15, no. 5, p. 155014771984711, May 2019, doi: 10.1177/1550147719847112.

[11] A. F. van Straten and B. C. Jobst, "Future of epilepsy treatment: integration of devices," vol. 9, pp. 587-599, 2014. 
[12] M. Tentori, L. Escobedo, and G. Balderas, "Smart Environment for children with Autism," 2015.

[13] A. M. Thomas et al., "Smart care spaces: needs for intelligent at-home care," International Journal of Space-Based and Situated Computing, vol. 3, no. 1, p. 35, 2013, doi: 10.1504/IJSSC.2013.051988.

[14] B. Mesko, "The Guide to the Future of Medicine," The Guide to the future of Medicine, pp. 1-25, 2014, doi: 10.1038/scientificamerican0413-48.

[15] V.-M. Teohari, C. Ungureanu, V. Bui, J. Arends, and R. M. Aarts, "Epilepsy Seizure Detection App for Wearable Technologies."

[16] T. Ohiwa, H. Yamamoto, and K. Yamazaki, "Sensor system to advice health-aware information for elderly people on daily living activities," in 2016 International Conference on Computing, Networking and Communications, ICNC 2016, 2016, doi: 10.1109/ICCNC.2016.7440556.

[17] E. I. Georga et al., "Development of a smart environment for diabetes data analysis and new knowledge mining," pp. 112-115, 2014, doi: 10.1109/MOBIHEALTH.2014.7015922.

[18] P. Nedungadi, A. Jayakumar, and R. Raman, "Personalized Health Monitoring System for Managing Well-Being in Rural Areas," Journal of Medical Systems, vol. 42, no. 1, Jan. 2018, doi: 10.1007/s10916-017-08549.

[19] D. J. Cook, M. Schmitter-edgecombe, and P. Dawadi, "Analyzing Activity Behavior and Movement in a Naturalistic Environment using Smart Home Techniques."

[20] S. N. Abdulkader, A. Atia, and M. S. M. Mostafa, "Brain computer interfacing: Applications and challenges," Egyptian Informatics Journal, vol. 16, no. 2. Elsevier B.V., pp. 213-230, 01-Jul-2015, doi: 10.1016/j.eij.2015.06.002.

[21] M. Singh and N. Jain, "Performance and evaluation of smartphone based wireless blood pressure monitoring system using Bluetooth," IEEE Sensors Journal, vol. 16, no. 23, pp. 8322-8328, Dec. 2016, doi: 10.1109/JSEN.2016.2597289.

[22] A. van de Vel, K. Verhaert, and B. Ceulemans, "Critical evaluation of four different seizure detection systems tested on one patient with focal and generalized tonic and clonic seizures," Epilepsy and Behavior, vol. 37, pp. 91-94, 2014, doi: 10.1016/j.yebeh.2014.06.014.

[23] O. Ositelu, J. S. Landy, B. Kadry, and A. Macario, "Smart Device Use Among Resident Physicians at Stanford Hospital," Cureus, Dec. 2012, doi: 10.7759/cureus.73. 
T. D. Aungst, "Medical applications for pharmacists using mobile devices," Annals of Pharmacotherapy, vol. 47, no. 7-8, pp. 1088-1095, Jul. 2013, doi: 10.1345/aph.1S035.

[25] "Groundbreaking Announcements as National Epilepsy Awareness Month Begins.” [Online]. Available: https://www.prnewswire.com/newsreleases/groundbreaking-announcements-as-national-epilepsy-awarenessmonth-begins-300176433.html. [Accessed: 30-Nov-2019].

[26] "Study highlights pros and cons of seizure detection technology| Epilepsy Society." [Online]. Available: https://www.epilepsysociety.org.uk/News/Study-highlights-pros-andcons-of-seizure-detection-technology-01-02-2016\#.XeJzOOj7TIW.

[Accessed: 30-Nov-2019].

[27] "Guardian \& Companion - Epilepsy Solutions: Epilepsy Solutions." [Online]. Available: http://epilepsysolutions.co.uk/guardian-companion/. [Accessed: 30-Nov-2019].

[28] "Technology and medicine." [Online]. Available: http://broughttolife.sciencemuseum.org.uk/broughttolife/themes/technolog ies. [Accessed: 25-Jan-2020].

[29] P. Bonato, "Wearable sensors and systems," IEEE Engineering in Medicine and Biology Magazine, vol. 29, no. 3, pp. 25-36, May 2010, doi: 10.1109/MEMB.2010.936554.

[30] E. Magiorkinis, A. Diamantis, K. Sidiropoulou, and C. Panteliadis, "Highights in the History of Epilepsy: The Last 200 Years," Epilepsy Research and Treatment, vol. 2014, pp. 1-13, 2014, doi: $10.1155 / 2014 / 582039$.

[31] “(PDF) The Role of Technology in Clinical Neuropsychology.” [Online]. Available:

https://www.researchgate.net/publication/316014475_The_Role_of_Tech nology_in_Clinical_Neuropsychology. [Accessed: 06-Feb-2020].

[32] V. J. Aski, S. S. Sonawane, and U. Soni, "IoT Enabled Ubiquitous Healthcare Data Acquisition and Monitoring System for Personal and Medical Usage Powered by Cloud Application: An Architectural Overview," 2019, pp. 1-15.

[33] M. Chen, Y. Ma, S. Ullah, W. Cai, and E. Song, "ROCHAS: Robotics and Cloud-assisted Healthcare System for Empty Nester.”

[34] A. W. Mitas, M. Rudzki, M. Skotnicka, and P. Lubina, "Activity Monitoring of the Elderly for Telecare Systems - Review," 2014, pp. 125138. 

clinical epidemiology," Clinical Epidemiology, vol. 9. Dove Medical Press Ltd, pp. 167-178, 16-Mar-2017, doi: 10.2147/CLEP.S106258.

[36] M. Chen, Y. Zhang, Y. Li, M. M. Hassan, and A. Alamri, "AIWAC: Affective interaction through wearable computing and cloud technology," IEEE Wireless Communications, vol. 22, no. 1, pp. 20-27, Feb. 2015, doi: 10.1109/MWC.2015.7054715.

[37] X. Huang and P. M. Matricardi, "Allergy and Asthma Care in the Mobile Phone Era," Clinical Reviews in Allergy and Immunology, vol. 56, no. 2. Humana Press Inc., pp. 161-173, 15-Apr-2019, doi: 10.1007/s12016-0168542-y.

[38] “(PDF) Semantic Web Application for Health Care.” [Online]. Available: https://www.researchgate.net/publication/284724192_Semantic_Web_Ap plication_for_Health_Care. [Accessed: 01-Dec-2019].

[39] X. Zenuni, B. Raufi, F. Ismaili, and J. Ajdari, "State of the Art of Semantic Web for Healthcare," Procedia - Social and Behavioral Sciences, vol. 195, pp. 1990-1998, Jul. 2015, doi: 10.1016/j.sbspro.2015.06.213.

[40] N. al Haider, S. Abidi, W. van Woensel, and S. S. R. Abidi, "Integrating existing large scale medical laboratory data into the semantic web framework," in Proceedings - 2014 IEEE International Conference on Big Data, IEEE Big Data 2014, 2015, pp. 1040-1048, doi: 10.1109/BigData.2014.7004338.

[41] M. Dragoni, M. Rospocher, T. Bailoni, R. Maimone, and C. Eccher, "Semantic technologies for healthy lifestyle monitoring," in Lecture Notes in Computer Science (including subseries Lecture Notes in Artificial Intelligence and Lecture Notes in Bioinformatics), 2018, vol. 11137 LNCS, pp. 307-324, doi: 10.1007/978-3-030-00668-6_19.

[42] E. Adel, S. El-Sappagh, S. Barakat, and M. Elmogy, "A unified fuzzy ontology for distributed electronic health record semantic interoperability," in U-Healthcare Monitoring Systems, Elsevier, 2019, pp. 353-395.

[43] N. v. Chawla and D. A. Davis, "Bringing big data to personalized healthcare: A patient-centered framework," Journal of General Internal Medicine, vol. 28, no. SUPPL.3. Sep-2013, doi: 10.1007/s11606-013-24558.

[44] A. Carbonaro, F. Piccinini, and R. Reda, "Semantic Description of Healthcare Devices to Enable Data Integration," in Advances in Intelligent Systems and Computing, 2018, vol. 738, pp. 627-630, doi: 10.1007/978-3319-77028-4_80. 

and Knowledge Management System on Epilepsy and Epileptic Seizures," Dec. 2010.

[46] M. Ntekouli et al., "A mapping of epilepsy's evolution: implementation of the proposed knowledge based model," Evolving Systems, vol. 9, no. 4, pp. 299-313, Dec. 2018, doi: 10.1007/s12530-017-9198-4.

[47] U. G. Hofmann, "Development of a Closed Loop Stimulation System for Epilepsy Therapy," no. October 2015, 2014.

[48] B. Direito, C. Teixeira, B. Ribeiro, M. Castelo-Branco, F. Sales, and A. Dourado, "Modeling epileptic brain states using EEG spectral analysis and topographic mapping," Journal of Neuroscience Methods, vol. 210, no. 2, pp. 220-229, Sep. 2012, doi: 10.1016/j.jneumeth.2012.07.006.

[49] S. Xie and S. Krishnan, "Wavelet-based sparse functional linear model with applications to EEGs seizure detection and epilepsy diagnosis," Medical and Biological Engineering and Computing, vol. 51, no. 1-2, pp. 49-60, Feb. 2013, doi: 10.1007/s11517-012-0967-8.

[50] S. Majumder, T. Mondal, and M. Deen, "Wearable Sensors for Remote Health Monitoring," Sensors, vol. 17, no. 12, p. 130, Jan. 2017, doi: 10.3390/s17010130.

[51] S. Sareen, S. K. Sood, and S. K. Gupta, "An Automatic Prediction of Epileptic Seizures Using Cloud Computing and Wireless Sensor Networks," Journal of Medical Systems, vol. 40, no. 11, Nov. 2016, doi: 10.1007/s10916-016-0579-1.

[52] "Epilepsy Phone Application | Seizure detector." [Online]. Available: http://www.epdetect.com/. [Accessed: 01-Dec-2019].

[53] N. A. Marzuki, W. Husain, and A. M. Shahiri, "MyEpiPal: Mobile application for managing, monitoring and predicting epilepsy patient," in Advances in Intelligent Systems and Computing, 2017, vol. 538 AISC, pp. 383-392, doi: 10.1007/978-3-319-49073-1_42.

[54] "Epilepsy Foundation My Seizure Diary | Epilepsy Foundation.” [Online]. Available: https://www.epilepsy.com/living-epilepsy/epilepsy-foundationmy-seizure-diary. [Accessed: 06-Feb-2020].

[55] "Mobile Patient Diaries: EpiDiary:: Irody." [Online]. Available: http://www.irody.com/mobile-patient-diaries/. [Accessed: 01-Dec-2019].

[56] “Alert for Embrace watch - Apps on Google Play." [Online]. Available: https://play.google.com/store/apps/details?id=com.empatica.embrace.alert \&hl=en_GB. [Accessed: 01-Dec-2019].

[57] "EmbracePlus | Empowering Breakthroughs in Neurology Research | Empatica." [Online]. Available: https://www.empatica.com/embraceplus/. [Accessed: 17-Jan-2020]. 

Kesavamurthy, and M. M. Mehndiratta, "Application of mobile phones in epilepsy care," International Journal of Epilepsy, vol. 2, no. 1. Reed Elsevier India Pvt. Ltd., pp. 28-37, 01-Jan-2015, doi: 10.1016/j.ijep.2015.02.002.

[59] "Vodafone whitepaper hails role of IoT in delivering personalised healthcare treatments." [Online]. Available: https://www.computerweekly.com/news/450426169/Vodafonewhitepaper-hails-role-of-IoT-in-delivering-personalised-healthcaretreatments. [Accessed: 02-Dec-2019].

[60] M. S. Sarif Ullah Patwary, "Smart Textiles and Nano-Technology: A General Overview," Journal of Textile Science \& Engineering, vol. 05, no. 01, 2015, doi: 10.4172/2165-8064.1000181.

[61] J. Qi, P. Yang, G. Min, O. Amft, F. Dong, and L. Xu, “Advanced internet of things for personalised healthcare systems: A survey," Pervasive and Mobile Computing, vol. 41. Elsevier B.V., pp. 132-149, 01-Oct-2017, doi: 10.1016/j.pmcj.2017.06.018.

[62] T. Sharon, "Self-Tracking for Health and the Quantified Self: ReArticulating Autonomy, Solidarity, and Authenticity in an Age of Personalized Healthcare," Philosophy \& Technology, vol. 30, no. 1, pp. $93-$ 121, Mar. 2017, doi: 10.1007/s13347-016-0215-5.

[63] I. S. Khayal and A. M. Farid, "Architecting a System Model for Personalized Healthcare Delivery and Managed Individual Health Outcomes," Complexity, vol. 2018, 2018, doi: 10.1155/2018/8457231.

[64] S. Vashist, E. Schneider, and J. Luong, "Commercial Smartphone-Based Devices and Smart Applications for Personalized Healthcare Monitoring and Management," Diagnostics, vol. 4, no. 3, pp. 104-128, Aug. 2014, doi: 10.3390/diagnostics4030104.

[65] M. Ivanovic and M. Semnic, "The Role of Agent Technologies in Personalized Medicine," in 2018 5th International Conference on Systems and Informatics, ICSAI 2018, 2019, pp. 299-304, doi: 10.1109/ICSAI.2018.8599421.

[66] "Over 40 Different Types Of Seizures - Revised | Epilepsy Foundation." [Online]. Available: https://www.epilepsy.com/connect/forums/productsresources-helpful-links/over-40-different-types-seizures-revised.

[Accessed: 02-Dec-2019].

[67] M. Pediaditis, M. Tsiknakis, V. Kritsotakis, M. Góralczyk, S. Voutoufianakis, and P. Vorgia, "Exploiting advanced video analysis technologies for a smart home monitoring platform for epileptic patients: Technological and legal preconditions," in 2012 International Conference 
on Telecommunications and Multimedia, TEMU 2012, 2012, pp. 202-207, doi: 10.1109/TEMU.2012.6294719.

"A vision of personalising medicine for epilepsy patients | Horizon 2020." [Online].

Available:

https://ec.europa.eu/programmes/horizon2020/en/news/visionpersonalising-medicine-epilepsy-patients. [Accessed: 02-Dec-2019].

[69] “Types of Seizures | Epilepsy Foundation." [Online]. Available: https://www.epilepsy.com/learn/types-seizures. [Accessed: 06-Feb-2020].

[70] "Seizures - Epilepsy Canada." [Online]. Available: https://www.epilepsy.ca/seizures.html. [Accessed: 06-Feb-2020].

[71] J. Bidwell, T. Khuwatsamrit, B. Askew, J. A. Ehrenberg, and S. Helmers, "Seizure reporting technologies for epilepsy treatment: A review of clinical information needs and supporting technologies," Seizure, vol. 32. W.B. Saunders Ltd, pp. 109-117, 01-Nov-2015, doi: 10.1016/j.seizure.2015.09.006.

[72] "Usage of bio-signals to detect epileptic seizures - Health Research Authority." [Online]. Available: https://www.hra.nhs.uk/planning-andimproving-research/application-summaries/research-summaries/usage-ofbio-signals-to-detect-epileptic-seizures/. [Accessed: 02-Dec-2019].

[73] "NIESM - Non-Invasive Epilepsy Monitoring System." [Online]. Available: http://www.niesm.com/. [Accessed: 02-Dec-2019].

[74] A. van de Vel et al., "Non-EEG seizure detection systems and potential SUDEP prevention: State of the art: Review and update," Seizure: European Journal of Epilepsy, vol. 41, pp. 141-153, 2016, doi: 10.1016/j.seizure.2016.07.012.

[75] N. F. Noy and D. L. Mcguinness, "Ontology Development 101: A Guide to Creating Your First Ontology."

[76] V. Boeva, M. Angelova, and E. Tsiporkova, "A Split-Merge Evolutionary Clustering Algorithm,” doi: 10.5220/0007573103370346.

[77] V. Boeva, M. Angelova, V. M. Devagiri, and E. Tsiporkova, "Patient Profiling Using Evolutionary Clustering,” 2019, doi: 10.1145/nnnnnnn.nnnnnnn.

[78] M. R. Crawford et al., "Characterization of patients who present with insomnia: Is there room for a symptom cluster-based approach?," Journal of Clinical Sleep Medicine, vol. 13, no. 7, pp. 911-921, 2017, doi: 10.5664/jcsm.6666.

[79] “2019/20 National Tariff Payment System-A consultation notice: Annex DtE Technical guidance for mental health clusters A joint publication by NHS England and NHS Improvement National Tariff Payment System-A consultation notice: Annex DtE Technical guidance for mental health 
clusters A joint publication by NHS England and NHS Improvement," 2019.

[80] "Supporting Information: Adult Mental Health Clustering Tool." [Online].

Available: https://www.datadictionary.nhs.uk/data_dictionary/nhs_business_definitio ns/a/adult_mental_health_clustering_tool_de.asp?shownav=1. [Accessed: 26-Jan-2020].

[81] S. Khalid, M. S. Ali, and D. Prieto-Alhambra, "Cluster Analysis to Detect Patterns of Drug Use from Routinely Collected Medical Data," in Proceedings - IEEE Symposium on Computer-Based Medical Systems, 2018, vol. 2018-June, pp. 194-198, doi: 10.1109/CBMS.2018.00041.

[82] T. Ahmad et al., "Clinical Implications of Cluster Analysis-Based Classification of Acute Decompensated Heart Failure and Correlation with Bedside Hemodynamic Profiles," PLOS ONE, vol. 11, no. 2, p. e0145881, Feb. 2016, doi: 10.1371/journal.pone.0145881.

[83] K. J. Cios and L. A. Kurgan, "Trends in Data Mining and Knowledge Discovery," in Advanced Techniques in Knowledge Discovery and Data Mining, Springer London, 2007, pp. 1-26.

[84] M. S. Garnett, T. Attwood, C. Peterson, and A. B. Kelly, "Autism spectrum conditions among children and adolescents: A new profiling tool," Australian Journal of Psychology, vol. 65, no. 4, pp. 206-213, Dec. 2013, doi: 10.1111/ajpy.12022.

[85] "Advances in Artificial Intelligence, Software and Systems Engineering Joint Proceedings of the AHFE 2018 International Conference on Human Factors in Artificial Intelligence and Social Computing, Software and Systems Engineering, The Human Side of Service Engineering and Human Factors in Energy, July 21-25, 2018, Loews Sapphire Falls Resort at Universal Studios, Orlando, Florida, USA | Tareq Ahram | Springer." [Online]. Available: https://www.springer.com/gp/book/9783319942285. [Accessed: 06-Feb-2020].

[86] J. R. Hobbs et al., "FASTUS: A Cascaded Finite-State Transducer for Extracting Information from Natural-Language Text."

[87] P. Barnaghi and C. H. Foh, "EEEM048-Internet of Things."

[88] Application development and design: Concepts, methodologies, tools, and applications. IGI Global, 2017.

[89] M. Boicu, G. Tecuci, B. Stanescu, G. C. Balan, and E. Popovici, "Ontologies and the Knowledge Acquisition Bottleneck."

[90] L. C. Tsoi, R. Patel, W. Zhao, and W. J. Zheng, "Text-mining approach to evaluate terms for ontology development," Journal of Biomedical 
Informatics, vol. 42, no. 5, pp. 824-830, Oct. 2009, doi: 10.1016/j.jbi.2009.03.009.

[91] D. S. Rajput, P. K. Singh, and M. Bhattacharya, "Reduct and variance based clustering of high dimensional dataset," in Lecture Notes in Computer Science (including subseries Lecture Notes in Artificial Intelligence and Lecture Notes in Bioinformatics), 2012, vol. 6411 LNCS, pp. 75-79, doi: 10.1007/978-3-642-27872-3_11.

[92] N. Fridman Noy and C. D. Hafner, "The State of the Art in Ontology Design," AI Magazine, vol. 18, no. 3, pp. 53-74, 1997, doi: 10.1609/aimag.v18i3.1306.

[93] V. Mirzaee, L. Iverson, and B. Hamidzadeh, "Towards Ontological Modelling of Historical Documents."

[94] M. Poveda-Villalón, M. Carmen Suárez-Figueroa, M. Ángel GarcíaDelgado, and A. Gómez-Pérez, "OOPS! (OntOlogy Pitfall Scanner!): supporting ontology evaluation on-line," IOS Press, 2009.

[95] T. Sampalli, M. Shepherd, and J. Duffy, "A patient profile ontology in the heterogeneous domain of complex and chronic health conditions," in Proceedings of the Annual Hawaii International Conference on System Sciences, 2011, doi: 10.1109/HICSS.2011.21.

[96] "Software Engineering and Computer Systems, Part III: Second International Conference, ICSECS 2011, Kuantan, Pahang, Malaysia, June 27-29, 2011, Proceedings | Request PDF." [Online]. Available: https://www.researchgate.net/publication/225304507_Software_Engineeri ng_and_Computer_Systems_Part_III_Second_International_Conference_I CSECS_2011_Kuantan_Pahang_Malaysia_June_27-

29_2011_Proceedings. [Accessed: 09-Dec-2019].

[97] “(PDF) Requirements Specification and Analysis for Embedded Systems." [Online]. Available: https://www.researchgate.net/publication/258341008_Requirements_Spec ification_and_Analysis_for_Embedded_Systems. [Accessed: 06-Feb2020].

[98] R. B. Johnson and A. J. Onwuegbuzie, "Toward a Definition of Mixed Methods Research," Journal of Mixed Methods Research, vol. 1, no. 2, pp. 112-133, 2007, doi: 10.1177/1558689806298224.

[99] R. S. Fisher et al., "Instruction manual for the ILAE 2017 operational classification of seizure types," Epilepsia, vol. 58, no. 4, pp. 531-542, Apr. 2017, doi: 10.1111/epi.13671.

[100] "(PDF) Building Ontology in Public Administration: A Case Study." [Online].

Available: 
https://www.researchgate.net/publication/221466344_Building_Ontology _in_Public_Administration_A_Case_Study. [Accessed: 09-Dec-2019].

[101] Emergency and Disaster Management. IGI Global, 2018.

[102] "What is an ontology and why we need it." [Online]. Available: https://protege.stanford.edu/publications/ontology_development/ontology 101-noy-mcguinness.html. [Accessed: 10-Dec-2019].

[103] P. P. Rezaeiye and M. Gheisari, Performance Analysis of two sensor data storages. .

[104] M. U. hold, M. Gruninger, and M. Uschold Michael Gruninger, "Ontologies: Principles, Methods and Applications," 1996.

[105] E. Rosch and F. publised in, "Principles of Categorization."

[106] “OWL Web Ontology Language Reference." [Online]. Available: https://www.w3.org/TR/owl-ref/. [Accessed: 09-Dec-2019].

[107] A. Papasalouros, K. Kotis, and K. Kanaris, "Automatic generation of tests from domain and multimedia ontologies," Interactive Learning Environments, vol. 19, no. 1, pp. 5-23, Jan. 2011, doi: 10.1080/10494820.2011.528704.

[108] A. Ameen, K. R. Khan, and B. P. Rani, "Construction of university ontology," in Proceedings of the 2012 World Congress on Information and Communication Technologies, WICT 2012, 2012, pp. 39-44, doi: 10.1109/WICT.2012.6409047.

[109] "OWL Web Ontology Language Overview." [Online]. Available: https://www.w3.org/TR/owl-features/. [Accessed: 09-Dec-2019].

[110] R. v Belavkin, "Lecture 8: Ontologies."

[111] M. Horridge, S. Jupp, G. Moulton, A. Rector, R. Stevens, and C. Wroe, "A Practical Guide To Building OWL Ontologies Using Protégé 4 and COODE Tools Edition 1.1,” 2007.

[112] “Ontology Matching | Jérôme Euzenat | Springer.” [Online]. Available: https://www.springer.com/gp/book/9783642387203. [Accessed: 09-Dec2019].

[113] J. Z. Pan and I. Horrocks, "Web ontology reasoning with datatype groups," Lecture Notes in Computer Science (including subseries Lecture Notes in Artificial Intelligence and Lecture Notes in Bioinformatics), vol. 2870, pp. 47-63, 2003, doi: 10.1007/978-3-540-39718-2_4.

[114] O. Bodenreider, B. Smith, A. Kumar, and A. Burgun, "Investigating Subsumption in DL-Based Terminologies: A Case Study in SNOMED CT."

[115] L. G. Cowell and B. Smith, "Infectious Disease Ontology," in Infectious Disease Informatics, New York, NY: Springer New York, 2010, pp. 373395. 
[116] “protégé." [Online]. Available: https://protege.stanford.edu/. [Accessed: 07-Feb-2020].

[117] "OntoGraf - Protege Wiki." [Online]. Available: https://protegewiki.stanford.edu/wiki/OntoGraf. [Accessed: 09-Dec-2019].

[118] R. Sivakumar and P. v Arivoli, "ONTOLOGY VISUALIZATION PROTÉGÉ TOOLS-A REVIEW," International Journal of Advanced Information Technology (IJAIT), vol. 1, no. 4, 2011, doi: 10.5121/ijait.2011.1401.

[119] "World Wide Web Consortium (W3C)." [Online]. Available: https://www.w3.org/. [Accessed: 07-Feb-2020].

[120] B. Liu, J. Li, and Y. Zhao, "Repairing and reasoning with inconsistent and uncertain ontologies," Advances in Engineering Software, vol. 45, no. 1, pp. 380-390, 2012, doi: 10.1016/j.advengsoft.2011.10.015.

[121] G. Antoniou and F. van Harmelen, "Web Ontology Language: OWL," in Handbook on Ontologies, Berlin, Heidelberg: Springer Berlin Heidelberg, 2009, pp. 91-110.

[122] "What are Linked Data and Linked Open Data? - Ontotext." [Online]. Available: https://www.ontotext.com/knowledgehub/fundamentals/linkeddata-linked-open-data/. [Accessed: 10-Dec-2019].

[123] "The OWL API: A Java API for OWL Ontologies | www.semantic-webjournal.net." [Online]. Available: http://www.semantic-webjournal.net/content/owl-api-java-api-owl-ontologies. [Accessed: 09-Dec2019].

[124] The Description Logic Handbook. Cambridge University Press, 2007.

[125] M. Poveda Villalón, "Ontology Evaluation: a pitfall-based approach to ontology diagnosis," 2016.

[126] T. Berners-Lee, J. Hendler, and O. Lassila, "The semantic web," Scientific American, vol. 284, no. 5. pp. 34-43, May-2001, doi: 10.1038/scientificamerican0501-34.

[127] "NeOn Methodology for Building Ontology Networks: Specification, Scheduling and Reuse," 2010.

[128] Y. Sure, S. Staab, and R. Studer, "On-To-Knowledge Methodology (OTKM)," in Handbook on Ontologies, Berlin, Heidelberg: Springer Berlin Heidelberg, 2004, pp. 117-132.

[129] M. Poveda-Villalón, M. Carmen Suárez-Figueroa, M. Ángel GarcíaDelgado, and A. Gómez-Pérez, "OOPS! (OntOlogy Pitfall Scanner!): supporting ontology evaluation on-line," IOS Press, 2009.

[130] "Weka 3 - Data Mining with Open Source Machine Learning Software in Java." [Online]. Available: https://www.cs.waikato.ac.nz/ml/weka/. [Accessed: 09-Dec-2019]. 
[131] "Determine Collection Method «Pell Institute." [Online]. Available: http://toolkit.pellinstitute.org/evaluation-guide/collect-data/determinecollection-method/. [Accessed: 09-Dec-2019].

[132] "Conceptual Framework: A Step-by-Step Guide on How to Make One." [Online]. Available: https://simplyeducate.me/2015/01/05/conceptualframework-guide/. [Accessed: 09-Dec-2019].

"NCBO BioPortal." [Online]. Available: http://bioportal.bioontology.org/ontologies. [Accessed: 10-Dec-2019].

[134] S. S. Sahoo, G. Q. Zhang, and S. D. Lhatoo, "Epilepsy informatics and an ontology-driven infrastructure for large database research and patient care in epilepsy," Epilepsia, vol. 54, no. 8. pp. 1335-1341, Aug-2013, doi: 10.1111/epi.12211.

[135] “Ontology Development Methodology - HL7Wiki." [Online]. Available: https://wiki.hl7.org/index.php?title=Ontology_Development_Methodolog y\#Step_4._Define_the_classes_and_the_class_hierarchy. [Accessed: 10Dec-2019].

[136] "Ontologies and Semantic Annotation. Part 2: Developing an Ontology." [Online]. Available: https://medium.com/sciforce/ontologies-andsemantic-annotation-part-2-developing-an-ontology-eec37b44c94b.

[Accessed: 10-Dec-2019].

[137] “An organization ontology: First draft | Epimorphics." [Online]. Available: https://www.epimorphics.com/an-organization-ontology-firstdraft/. [Accessed: 10-Dec-2019].

[138] M. B. Sarder, S. Ferreira, J. Rogers, and D. H. Liles, "A methodology for design ontology modeling," in Portland International Conference on Management of Engineering and Technology, 2007, pp. 1011-1018, doi: 10.1109/PICMET.2007.4349422.

[139] "What is a Reference Concept? | AI3:::Adaptive Information." [Online]. Available: http://www.mkbergman.com/938/what-is-a-reference-concept/. [Accessed: 10-Dec-2019].

[140] "A Reference Guide to Ontology Best Practices | AI3:::Adaptive Information." [Online]. Available: http://www.mkbergman.com/911/areference-guide-to-ontology-best-practices/. [Accessed: 10-Dec-2019].

[141] "semantics - What is the meaning of 'Equivalent To' in Protege? - Stack Overflow." [Online].

Available: https://stackoverflow.com/questions/29452246/what-is-the-meaning-ofequivalent-to-in-protege. [Accessed: 10-Dec-2019].

[142] “K-Means Clustering: Numerical Example." [Online]. Available: https://people.revoledu.com/kardi/tutorial/kMean/NumericalExample.htm. [Accessed: 10-Dec-2019]. 
[143] "What are Ontologies and What are the Benefits of Using Ontologies." [Online].

Available:

https://www.ontotext.com/knowledgehub/fundamentals/what-areontologies/. [Accessed: 10-Dec-2019].

[144] K. Giri, "Role of Ontology in Semantic Web," 2011.

[145] “Starting Protégé — Ontology 101Tutorial 1.0 documentation.” [Online]. Available:

https://ontology101tutorial.readthedocs.io/en/latest/StartingProtege.html\#t argetText=Prot\%C3\%A9g\%C3\%A9\%20allows\%20you\%20to\%20save,to \%20work\%20with\%20in\%20Prot\%C3\%A9g\%C3\%A9.\&targetText=Cho ose $\% 20$ a\%20location $\% 20$ on\%20your\%20computer\%20to\%20save $\% 20$ yo ur\%20ontology. [Accessed: 10-Dec-2019].

[146] M. Uschold and R. Jasper, "A Framework for Understanding and Classifying Ontology Applications Proceedings of the IJCAI-99 workshop on Ontologies and Problem-Solving Methods (KRR5)," 1999.

[147] I. Horrocks, P. F. Patel-Schneider, and F. van Harmelen, "From SHIQ and RDF to OWL: The making of a Web Ontology Language," Web Semantics, vol. 1, no. 1, pp. 7-26, 2003, doi: 10.1016/j.websem.2003.07.001.

[148] "Is Ontology File created in Protege with RDF/XML Format a RDF file or the OWL file? - Stack Overflow." [Online]. Available: https://stackoverflow.com/questions/24288133/is-ontology-file-created-inprotege-with-rdf-xml-format-a-rdf-file-or-the-owl-fi. [Accessed: 10-Dec2019].

[149] H. Guo, S. Gao, J. Krogstie, H. Trætteberg, and A. I. Wang, "An evaluation of ontology based domain analysis for model driven development," International Journal on Semantic Web and Information Systems, vol. 11, no. 4, pp. 41-63, Oct. 2015, doi: 10.4018/IJSWIS.2015100102.

[150] "All about Categorical Variable Encoding - Towards Data Science." [Online]. Available: https://towardsdatascience.com/all-about-categoricalvariable-encoding-305f3361fd02. [Accessed: 10-Dec-2019].

[151] A. Gangemi, C. Catenacci, M. Ciaramita, and J. Lehmann, "Modelling Ontology Evaluation and Validation," 2006, pp. 140-154.

[152] “OOPS! - OntOlogy Pitfall Scanner!" [Online]. Available: http://oops.linkeddata.es/advanced.jsp. [Accessed: 10-Dec-2019].

[153] "D7.1.3-Study on persistent URIs, with identification of best practices and recommendations on the topic for the MSs and the EC Deliverable."

[154] "Creative Commons - Attribution 4.0 International - CC BY 4.0." [Online].

Available: 
https://creativecommons.org/licenses/by/4.0/legalcode. [Accessed: 10Dec-2019].

[155] S. Windgassen, R. Moss-Morris, K. Goldsmith, and T. Chalder, "The importance of cluster analysis for enhancing clinical practice: an example from irritable bowel syndrome," Journal of Mental Health, vol. 27, no. 2. Taylor and Francis Ltd, pp. 94-96, 04-Mar-2018, doi: 10.1080/09638237.2018.1437615.

[156] E. Ahlqvist et al., "Novel subgroups of adult-onset diabetes and their association with outcomes: a data-driven cluster analysis of six variables," The Lancet Diabetes and Endocrinology, vol. 6, no. 5, pp. 361-369, May 2018, doi: 10.1016/S2213-8587(18)30051-2.

[157] P. Haldar et al., "Cluster Analysis and Clinical Asthma Phenotypes," American Journal of Respiratory and Critical Care Medicine, vol. 178, no. 3, pp. 218-224, Aug. 2008, doi: 10.1164/rccm.200711-1754OC.

[158] S. R. Newcomer, J. F. Steiner, and E. A. Bayliss, "Identifying subgroups of complex patients with cluster analysis," American Journal of Managed Care, vol. 17, no. 8, Aug. 2011.

[159] P. E. Green, R. E. Frank, and P. J. Robinson, "Cluster Analysis in Test Market Selection," Management Science, vol. 13. INFORMS, doi: $10.2307 / 2627665$.

[160] "K-means Algorithm g Cluster Analysis in Data Mining."

[161] L. Rokach and O. Maimon, "Clustering Methods," in Data Mining and Knowledge Discovery Handbook, Springer-Verlag, 2006, pp. 321-352.

[162] "Data Mining: Concepts and Techniques - Jiawei Han, Jian Pei, Micheline Kamber - Google Books." [Online]. Available: https://books.google.co.uk/books?hl=en\&lr=\&id=pQws07tdpjoC\&oi=fnd $\& p g=$ PP1 \&dq=J.+Han, + M.+Kamber, + and + J.+Pei, $+\%$ E2\%80\%9CData+ Mining:+Concepts+and+Techniques, $\%$ E2\% $80 \% 9 \mathrm{D}+$ Morgan+Kaufmann+ Publishers,+2011\&ots=tzLz_ZiA1Z\&sig=NZwOxNc_41i91E_XC-

BmZY-

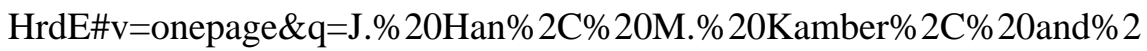
0J.\%20Pei\%2C\%20\%E2\%80\%9CData\%20Mining\%3A\%20Concepts\%2 0and\%20Techniques\%2C\%E2\%80\%9D\%20Morgan\%20Kaufmann\%20P ublishers\%2C\%202011\&f=false. [Accessed: 13-May-2020].

[163] V. Estivill-Castro and J. Yang, "Fast and robust general purpose clustering algorithms," Data Mining and Knowledge Discovery, vol. 8, no. 2, pp. 127-150, Mar. 2004, doi: 10.1023/B:DAMI.0000015869.08323.b3.

[164] M. K. Afridi, N. Azam, and J. T. Yao, "Variance based three-way clustering approaches for handling overlapping clustering," International 
Journal of Approximate Reasoning, vol. 118, pp. 47-63, Mar. 2020, doi: 10.1016/j.ijar.2019.11.011.

[165] N. Dhanachandra, Y. J. Chanu, and Kh. M. Singh, "A new hybrid image segmentation approach using clustering and black hole algorithm," Computational Intelligence, p. coin.12297, Mar. 2020, doi: 10.1111/coin.12297.

[166] A. Mukhopadhyay, U. Maulik, and S. Bandyopadhyay, "A survey of multiobjective evolutionary clustering," ACM Computing Surveys, vol. 47, no. 4, May 2015, doi: 10.1145/2742642.

[167] "How Many Clusters? Which Clustering Method? Answers Via ModelBased Cluster Analysis - OUP Journals \& Magazine.” [Online]. Available: https://ieeexplore.ieee.org/document/8129683. [Accessed: 13-May-2020].

[168] N. H. Shah and M. Mittal, Handbook of Research on Promoting Business Process Improvement Through Inventory Control Techniques. IGI Global, 2017.

[169] J. Kogan, C. Nicholas, and M. Teboulle, Grouping multidimensional data: Recent advances in clustering. Springer Berlin Heidelberg, 2006.

[170] C. Manu, "ANALYSIS OF CLUSTERING TECHNIQUE FOR CRM.”

[171] “Unsupervised Learning - MATLAB \& Simulink." [Online]. Available: https://uk.mathworks.com/discovery/unsupervised-learning.html. [Accessed: 10-Dec-2019].

[172] "Clustering techniques in data mining: A comparison - IEEE Conference Publication." [Online]. Available: https://ieeexplore.ieee.org/document/7100283. [Accessed: 10-Dec-2019].

[173] "What are the pros and cons of k-means vs. hierarchical clustering? Quora." [Online]. Available: https://www.quora.com/What-are-the-prosand-cons-of-k-means-vs-hierarchical-clustering. [Accessed: 10-Dec2019].

[174] J. H. Gennari, P. Langley, and D. Fisher, "Models of incremental concept formation," Artificial Intelligence, vol. 40, no. 1-3, pp. 11-61, 1989, doi: 10.1016/0004-3702(89)90046-5.

[175] H. Khanali and B. Vaziri, "A Survey on Clustering Algorithms for Partitioning Method," International Journal of Computer Applications, vol. 155, no. 4, pp. 20-25, Dec. 2016, doi: 10.5120/ijca2016912291.

[176] M. San, "Changelog: 12 Dec 2016 Advantages \& Disadvantages of k-Means and Hierarchical clustering (Unsupervised Learning) Machine Learning for Language Technology ML4LT (2016) 2016 Advantages \& Disadvantages of k--Means and Hierarchical Clustering."

[177] N. Singh and D. Singh, "Performance Evaluation of K-Means and Heirarichal Clustering in Terms of Accuracy and Running Time." 
[178] Y. Meng and X. Liu, "Application of K-means algorithm based on ant clustering algorithm in macroscopic planning of highway transportation hub," in Proceedings of the 2007 1st International Symposium on Information Technologies and Applications in Education, ISITAE 2007, 2007, pp. 483-488, doi: 10.1109/ISITAE.2007.4409331.

[179] N. Raghu Kisore and C. H. B Koteswaraiah, "Improving ATM coverage area using density based clustering algorithm and voronoi diagrams," Information Sciences, vol. 376, pp. 1-20, Jan. 2017, doi: 10.1016/j.ins.2016.09.058.

[180] X. Peng, C. Zhou, D. Hepburn, M. D. Judd, and W. H. Siew, “Application of K-Means method to pattern recognition in on-line cable partial discharge monitoring," IEEE Transactions on Dielectrics and Electrical Insulation, vol. 20, no. 3, pp. 754-761, 2013, doi: 10.1109/TDEI.2013.6518945.

[181] J. Vaidya and C. Clifton, "Privacy-preserving K-means clustering over vertically partitioned data," in Proceedings of the ACM SIGKDD International Conference on Knowledge Discovery and Data Mining, 2003, pp. 206-215, doi: 10.1145/956750.956776.

[182] Z. Erkin, T. Veugen, T. Toft, and R. L. Lagendijk, "Privacy-preserving distributed clustering," Eurasip Journal on Information Security, vol. 2013, no. 1, pp. 1-15, Nov. 2013, doi: 10.1186/1687-417X-2013-4.

[183] L. Ai, X. Gao, and J. Xiong, "Application of mean-shift clustering to Blood oxygen level dependent functional MRI activation detection," $B M C$ Medical Imaging, vol. 14, no. 1, p. 6, Feb. 2014, doi: 10.1186/1471-234214-6.

[184] S. Saha, A. K. Alok, and A. Ekbal, "Brain image segmentation using semi-supervised clustering," Expert Systems with Applications, vol. 52, pp. 50-63, Jun. 2016, doi: 10.1016/j.eswa.2016.01.005.

[185] M. Rastgarpour, J. Shanbehzadeh, and H. Soltanian-Zadeh, "A hybrid method based on fuzzy clustering and local region-based level set for segmentation of inhomogeneous medical images systems-level quality improvement," Journal of Medical Systems, vol. 38, no. 8, p. 68, Aug. 2014, doi: 10.1007/s10916-014-0068-3.

[186] A. Ghosal, A. Nandy, A. K. Das, S. Goswami, and M. Panday, "A Short Review on Different Clustering Techniques and Their Applications," in Advances in Intelligent Systems and Computing, 2020, vol. 937, pp. 69-83, doi: 10.1007/978-981-13-7403-6_9.

[187] A. Saxena et al., "A review of clustering techniques and developments," Neurocomputing, vol. 267, pp. 664-681, Dec. 2017, doi: 10.1016/j.neucom.2017.06.053. 
[188] H. Bensmail, G. Celeux, A. E. Raftery, and C. P. Robert, "Inference in model-based cluster analysis," Statistics and Computing, vol. 7, no. 1, pp. 1-10, 1997, doi: 10.1023/A:1018510926151.

[189] A. And and U. Pandey, "77 Anita," 2015.

[190] "Introduction to K-means Clustering | Oracle Data Science.” [Online]. Available: https://blogs.oracle.com/datascience/introduction-to-k-meansclustering. [Accessed: 10-Dec-2019].

[191] "ROSALIND | Glossary | Euclidean distance." [Online]. Available: http://rosalind.info/glossary/euclidean-distance/. [Accessed: 10-Dec2019].

[192] S. Jain, A. M. Afshar, and M. N. Doja, "K-MEANS CLUSTERING USING WEKA INTERFACE."

[193] V. Sudarsan and R. Sugumar, "Calculate Euclidean Distance for Distributed Environment Using Weka Code."

[194] "Importance of Distance Metrics in Machine Learning Modelling." [Online]. Available: https://towardsdatascience.com/importance-ofdistance-metrics-in-machine-learning-modelling-e51395ffe60d.

[Accessed: 10-Dec-2019].

[195] “Clustering Distance Measures - Datanovia." [Online]. Available: https://www.datanovia.com/en/lessons/clustering-distance-measures/. [Accessed: 10-Dec-2019].

[196] D. R. Garvey and J. Wesley Hines, "Robust distance measures for on-line monitoring: Why use Euclidean?," in Applied Artificial Intelligence Proceedings of the 7th International FLINS Conference, FLINS 2006, 2006, pp. 922-929, doi: 10.1142/9789812774118_0129.

[197] H. Hua, H. Xie, and E. Tanin, "Is Euclidean Distance Really that Bad with Road Networks," p. 10, 2018, doi: 10.1145/3283207.3283215.

[198] I. Journal, "Predicting Students Academic Performance Using Education Data Mining," IJCSMC.

[199] “K-Means Clustering in WEKA." [Online]. Available: http://facweb.cs.depaul.edu/mobasher/classes/ect584/weka/k-means.html. [Accessed: 10-Dec-2019].

[200] "How To Estimate The Performance of Machine Learning Algorithms in Weka."[Online]. Available: https://machinelearningmastery.com/estimateperformance-machine-learning-algorithms-weka/. [Accessed: 10-Dec2019].

[201] "Intro to Machine Learning \& NLP with Python and Weka | Codementor." [Online]. Available: https://www.codementor.io/benjamincohen/intro-to-machine-learning-nlpwith-python-and-weka-argnk39jr. [Accessed: 11-Dec-2019]. 
https://www.analyticsvidhya.com/blog/2019/08/comprehensive-guide-kmeans-clustering/. [Accessed: 11-Dec-2019].

[203] "Determining The Optimal Number Of Clusters: 3 Must Know Methods

$$
\text { Datanovia." [Online]. }
$$

Available:

https://www.datanovia.com/en/lessons/determining-the-optimal-numberof-clusters-3-must-know-methods/\#elbow-method. [Accessed: 11-Dec2019].

[204] "How to Automatically Determine the Number of Clusters in your Data and more - Data Science Central." [Online]. Available: https://www.datasciencecentral.com/profiles/blogs/how-to-automaticallydetermine-the-number-of-clusters-in-your-dat. [Accessed: 11-Dec-2019].

[205] “Klaus Wassermann's Page - Data Science Central.” [Online]. Available: https://www.datasciencecentral.com/profile/KlausWassermamm.

[Accessed: 11-Dec-2019].

[206] "Error Sum of Squares." [Online]. Available: https://hlab.stanford.edu/brian/error_sum_of_squares.html. [Accessed: 11Dec-2019].

[207] P. Yildirim, L. Majnarić, O. I. Ekmekci, and A. Holzinger, "Knowledge discovery of drug data on the example of adverse reaction prediction," $B M C$ Bioinformatics, vol. 15, no. S6, p. S7, May 2014, doi: 10.1186/1471-210515-S6-S7.

[208] "Introduction to K-means Clustering - Dileka Madushan - Medium." [Online]. Available: https://medium.com/@dilekamadushan/introductionto-k-means-clustering-7c0ebc997e00. [Accessed: 11-Dec-2019].

[209] "Kmeans with WEKA." [Online]. Available: https://forums.pentaho.com/threads/70648-Kmeans-with-WEKA/. [Accessed: 07-Feb-2020].

[210] E. Bruno et al., "Wearable technology in epilepsy: The views of patients, caregivers, and healthcare professionals," Epilepsy and Behavior, vol. 85, pp. 141-149, Aug. 2018, doi: 10.1016/j.yebeh.2018.05.044.

[211] A. Ulate-Campos, F. Coughlin, M. Gaínza-Lein, I. S. Fernández, P. L. Pearl, and T. Loddenkemper, "Automated seizure detection systems and their effectiveness for each type of seizure," Seizure, vol. 40. W.B. Saunders Ltd, pp. 88-101, 01-Aug-2016, doi: 10.1016/j.seizure.2016.06.008.

[212] "Sensors | Special Issue: Wearable Sensors in Healthcare: Methods, Algorithms, Applications.” [Online]. Available: 
https://www.mdpi.com/journal/sensors/special_issues/WSHMAA.

[Accessed: 22-Jan-2020].

[213] M. Ghamari, "A review on wearable photoplethysmography sensors and their potential future applications in health care," International Journal of Biosensors \& Bioelectronics, vol. 4, no. 4, p. 195, 2018, doi: 10.15406/ijbsbe.2018.04.00125.

[214] P. Jallon, S. Bonnet, M. Antonakios, and R. Guillemaud, "Detection system of motor epileptic seizures through motion analysis with 3D accelerometers," in Proceedings of the 31st Annual International Conference of the IEEE Engineering in Medicine and Biology Society: Engineering the Future of Biomedicine, EMBC 2009, 2009, pp. 2466-2469, doi: 10.1109/IEMBS.2009.5334770.

[215] W. J. C. van Elmpt, T. M. E. Nijsen, P. A. M. Griep, and J. B. A. M. Arends, "A model of heart rate changes to detect seizures in severe epilepsy," Seizure, vol. 15, no. 6, pp. 366-375, Sep. 2006, doi: 10.1016/j.seizure.2006.03.005.

[216] "Video-EEG Monitoring | Epilepsy Foundation.” [Online]. Available: https://www.epilepsy.com/learn/professionals/about-epilepsyseizures/classifying-seizures/nonepileptic-seizures/diagnosis-1. [Accessed: 24-Jan-2020].

[217] C. Viboud and M. Santillana, "Fitbit-informed influenza forecasts," The Lancet Digital Health, Jan. 2020, doi: 10.1016/S2589-7500(19)30241-9.

[218] "Focal Onset Seizures (Partial Seizures)." [Online]. Available: https://www.healthline.com/health/partial-focal-seizure. [Accessed: 07Feb-2020].

[219] M. Velez, R. S. Fisher, V. Bartlett, and S. Le, "Tracking generalized tonic-clonic seizures with a wrist accelerometer linked to an online database," Seizure, vol. 39, pp. 13-18, Jul. 2016, doi: 10.1016/j.seizure.2016.04.009.

[220] S. R. Gouravajhala and L. Khuon, "A multi-modality sensor platform approach to detect epileptic seizure activity," in 2012 38th Annual Northeast Bioengineering Conference, NEBEC 2012, 2012, pp. 233-234, doi: 10.1109/NEBC.2012.6207049.

[221] M. Z. Poh, T. Loddenkemper, N. C. Swenson, S. Goyal, J. R. Madsen, and R. W. Picard, "Continuous monitoring of electrodermal activity during epileptic seizures using a wearable sensor," in 2010 Annual International Conference of the IEEE Engineering in Medicine and Biology Society, EMBC'10, 2010, pp. 4415-4418, doi: 10.1109/IEMBS.2010.5625988.

[222] S. Tiwari, V. Sharma, M. Mujawar, Y. K. Mishra, A. Kaushik, and A. Ghosal, "Biosensors for epilepsy management: State-of-art and future 
aspects," Sensors (Switzerland), vol. 19, no. 7. MDPI AG, 01-Apr-2019, doi: 10.3390/s19071525.

[223] M. Maier, "Personalized medicine - a tradition in general practice!," European Journal of General Practice, vol. 25, no. 2. Taylor and Francis Ltd, pp. 63-64, 03-Apr-2019, doi: 10.1080/13814788.2019.1589806.

[224] "The PhD Blog: Top 10 Hints For Understanding Your Ontology, Epistemology and Methodology." [Online]. Available: http://doctoralstudy.blogspot.com/2017/01/top-10-hints-forunderstanding-your.html. [Accessed: 11-Dec-2019].

[225] "Decision Tree Algorithm — Explained - Towards Data Science." [Online]. Available: https://towardsdatascience.com/decision-treealgorithm-explained-83beb6e78ef4. [Accessed: 21-May-2020].

[226] "Classification and Regression Trees (CART) Theory and Applications," 2004.

[227] R. Meier, Professional Android 4 Application Development Wrox Professional Guides, 3rd, illustr ed. John Wiley \& Sons, 2012, 2012.

[228] E. Rusty. Harold, Java network programming. O’Reilly, 2005.

[229] "Wearable technology in epilepsy: The views of patients, caregivers, and healthcare professionals. Epilepsy \& Behavior - Google Search.” [Online]. Available:

https://www.google.com/search?q=Wearable+technology+in+epilepsy\%3 $\mathrm{A}+$ The+views+of+patients $\% 2 \mathrm{C}+$ caregivers $\% 2 \mathrm{C}+$ and + healthcare+professi onals.+Epilepsy+\%26+Behavior\&rlz=1C1CHBF_enGB758GB758\&oq= Wearable+technology+in+epilepsy\%3A+The+views+of+patients\%2C+ca regivers $\% 2 \mathrm{C}+$ and +healthcare+professionals.+Epilepsy+\%26+Behavior\&a $\mathrm{qs}=$ chrome..69i57.472j0j8\&sourceid=chrome\&ie=UTF-8. [Accessed: 20 May-2020].

[230] J. M. Peake, G. Kerr, and J. P. Sullivan, "A critical review of consumer wearables, mobile applications, and equipment for providing biofeedback, monitoring stress, and sleep in physically active populations," Frontiers in Physiology, vol. 9, no. JUN. Frontiers Media S.A., 28-Jun-2018, doi: 10.3389/fphys.2018.00743.

[231] “(PDF) From Seizure Detection to Prediction: A Review of Wearables and Related Devices Applicable to Epilepsy via Peripheral Measurements." [Online]. Available: https://www.researchgate.net/publication/340279429_From_Seizure_Dete ction_to_Prediction_A_Review_of_Wearables_and_Related_Devices_Ap plicable_to_Epilepsy_via_Peripheral_Measurements. [Accessed: 20-May2020]. 

https://azure.microsoft.com/en-gb/overview/iot/. [Accessed: 20-May2020].

[233] R. K. L. Ko, B. S. Lee, and S. Pearson, "Towards achieving accountability, auditability and trust in cloud computing," in Communications in Computer and Information Science, 2011, vol. 193 CCIS, no. PART 4, pp. 432-444, doi: 10.1007/978-3-642-22726-4_45.

[234] "IoT technologies and protocols | Microsoft Azure." [Online]. Available: https://azure.microsoft.com/en-gb/overview/internet-of-things-iot/iottechnology-protocols/. [Accessed: 20-May-2020].

[235] "The Power of Wearable Sensors in Healthcare -." [Online]. Available: https://thejournalofmhealth.com/the-power-of-wearable-sensors-inhealthcare/. [Accessed: 22-Jan-2020].

[236] “A Complete Guide to IoT Platforms - Yonomi." [Online]. Available: https://www.yonomi.co/complete-guide-to-iot-platforms. [Accessed: 21May-2020].

[237] "How Effective Performance Testing Can Help Build Robust IoT Systems." [Online]. Available: https://www.einfochips.com/blog/howeffective-performance-testing-can-help-build-robust-iot-systems/.

[Accessed: 21-May-2020].

[238] "Embrace Seizure Monitoring System by Empatica, USA." [Online]. Available: https://www.medicaldevice-network.com/projects/embraceseizure-monitoring-system/. [Accessed: 30-Nov-2019].

[239] "Flash glucose sensing available on NHS for type 1 diabetes from November 2017 - JDRF, the type 1 diabetes charity." [Online]. Available: https://jdrf.org.uk/news/flash-glucose-sensing-available-nhs-type-1diabetes/. [Accessed: 22-Jan-2020].

[240] "Engineers creating miniaturized, wireless oxygen sensor for sick infants: Mobile, wearable device the size of a Band-Aid could allow babies to leave the hospital and be monitored from home -- ScienceDaily." [Online]. Available:

https://www.sciencedaily.com/releases/2019/11/191114154454.htm. [Accessed: 22-Jan-2020].

[241] F. Porciuncula et al., "Wearable Movement Sensors for Rehabilitation: A Focused Review of Technological and Clinical Advances," PM and R, vol. 10, no. 9. Elsevier Inc., pp. S220-S232, 01-Sep-2018, doi: 10.1016/j.pmrj.2018.06.013. 


\section{Appendix A}

\section{Backend Monitoring}

Raw accelerometer and heart-rate data is accessed from the Fitbit Ionic using the devices 'API'. A custom made 'APP' that runs on the watch is used for this purpose. The monitoring data is then sent from the device to the mobile phone which it's paired, and from there to a remote private server. The sensors are connected to an Android mobile phone with Bluetooth. The custom made 'APP', is named EM (Effects Monitor), shown in the image below. The main functions of the EM App are:

- To capture and store data while with the user.

- To stores the captured data while having no access to the mobile device.

On start-up, the EM app starts to sense and capture heart-rate/heart-rate and accelerometer data and stores it to memory. This takes place without necessity for access to the internet. Once the device can gain access to the internet, it auto-uploads the data to the remote private server.

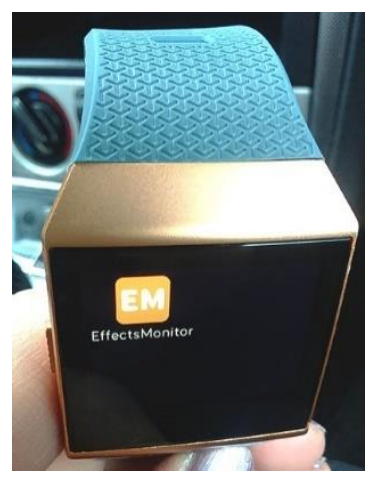

When the monitoring data is sent to the remote private server it is displayed. The program pulls the data from Fitbit Ionic device and displays it on the web page shown in the image below.

The EM APP contains the modules to read and process the monitoring data.

\begin{tabular}{|c|c|c|c|c|c|c|c|}
\hline ID & Fitbit & D Capture Date/Time & Heart Rate & $\operatorname{Acc} x$ & Acc $\mathrm{Y}$ & Acc $z$ & Entry Time \\
\hline 8029 & A & Wed Feb 062019 19:25:01 GMT $+00: 00$ & 70 & -4.270188331604 & -9.3328919274902 & 0.28950936989648 & 2019-02-06 19:27:39 \\
\hline 8004 & A & Wed Feb 062019 19:25:01 GMT $+00: 00$ & 70 & -4.270188331604 & -9.3328919274902 & 0.28950936889648 & 2019-02-06 19:26:57 \\
\hline 8028 & A & Wed Feb $06201919: 24: 55 \mathrm{GMT}+00: 00$ & 70 & -5.2231101989746 & -8.5870656967163 & -1.1983337402344 & 2019-02-06 19:27:39 \\
\hline 8003 & A & Wed Feb 062019 19:24:55 GMT +00:00 & 70 & -5.2231101989746 & -8.5870656967163 & -1.1983337402344 & $2019-02-06 \quad 19: 26: 57$ \\
\hline 8002 & A & Wed Feb 062019 19:24:50 GMT+00:00 & 70 & -3.227481842041 & -9.8775796990259 & -1.8974618911743 & 2019-02-06 19:26:56 \\
\hline 8027 & A & Wed Feb 062019 19:24:50 GMT $+00: 00$ & 70 & -3.227481842041 & -9.8775796990259 & -1.8974618911743 & $2019-02-06 \quad 19: 27: 38$ \\
\hline
\end{tabular}




\section{Appendix B}

\section{Hospital Patient Observations}

\section{Hospital Patient 1 Information}

\begin{tabular}{|l|l|}
\hline Patient Profile: & HP1 \\
\hline Seizure Type: & $\begin{array}{l}\text { FAS (Focal Aware Seizure) also known as } \\
\text { 'aura's } \\
\text { FSIA (Focal seizures with loss of awareness.) }\end{array}$ \\
\hline Dominant Side: & Right \\
\hline Time of Day: & Diurnal \\
\hline Trigger: & Unknown \\
\hline Key Symptoms: & $\begin{array}{l}\text { LOC, Automatisms (seizures usually lasting 10-20 } \\
\text { seconds) }\end{array}$ \\
\hline
\end{tabular}

\section{$>$ Observation Criteria}

\begin{tabular}{|c|c|}
\hline Observation & Criteria \\
\hline Observation 10 & $\begin{array}{l}\text { Seizure occurs with sensor positioned on } \\
\text { right wrist }(09.44 \mathrm{am})\end{array}$ \\
\hline Observation 11 & $\begin{array}{l}\text { Seizure occurs with sensor positioned on } \\
\text { right wrist }(20.36 \mathrm{pm})\end{array}$ \\
\hline Observation 12 & $\begin{array}{l}\text { Seizure occurs with sensor positioned on } \\
\text { right wrist }(21.06 \mathrm{pm})\end{array}$ \\
\hline Observation a HP1 & $\begin{array}{l}\text { Seizure occurs with sensor positioned on } \\
\text { right wrist }(09.55 \mathrm{am})\end{array}$ \\
\hline Observation b HP1 & $\begin{array}{l}\text { Seizure occurs with sensor positioned on } \\
\text { right wrist }(10.02 \mathrm{am})\end{array}$ \\
\hline Observation c HP1 & $\begin{array}{l}\text { Seizure occurs with sensor positioned on } \\
\text { right wrist }(19.26)\end{array}$ \\
\hline Observation d HP1 & $\begin{array}{l}\text { Seizure occurs with sensor positioned on } \\
\text { right wrist (20.17) }\end{array}$ \\
\hline Observation e HP1 & $\begin{array}{l}\text { Seizure occurs with sensor positioned on } \\
\text { right wrist }(21.00)\end{array}$ \\
\hline Observation f HP1 & $\begin{array}{l}\text { Seizure occurs with sensor positioned on } \\
\text { right wrist (11.01) }\end{array}$ \\
\hline Observation g HP1 & $\begin{array}{l}\text { Seizure occurs with sensor positioned on } \\
\text { right wrist }(20.44)\end{array}$ \\
\hline Observation h HP1 & $\begin{array}{l}\text { Seizure occurs with sensor positioned on } \\
\text { right wrist }(20.50)\end{array}$ \\
\hline Observation 13 & $\begin{array}{l}\text { No seizure occurs with sensor positioned } \\
\text { on right wrist during day-time }\end{array}$ \\
\hline Observation 14 & $\begin{array}{l}\text { No seizure occurs with sensor positioned } \\
\text { on right wrist during night-time }\end{array}$ \\
\hline Observation 15 & $\begin{array}{l}\text { No seizure occurs with sensor positioned } \\
\text { on right wrist during night-time }\end{array}$ \\
\hline Observation 16 & $\begin{array}{l}\text { No seizure occurs with sensor positioned } \\
\text { on right wrist during night-time }\end{array}$ \\
\hline Observation 17 & $\begin{array}{l}\text { No seizure occurs with sensor positioned } \\
\text { on right wrist during night-time }\end{array}$ \\
\hline
\end{tabular}


$>$ Results

Observation 10: Seizure occurs with sensor positioned on right wrist

Observation Time: 09.43.-09-46am

Seizure Occurrence: 09.44am

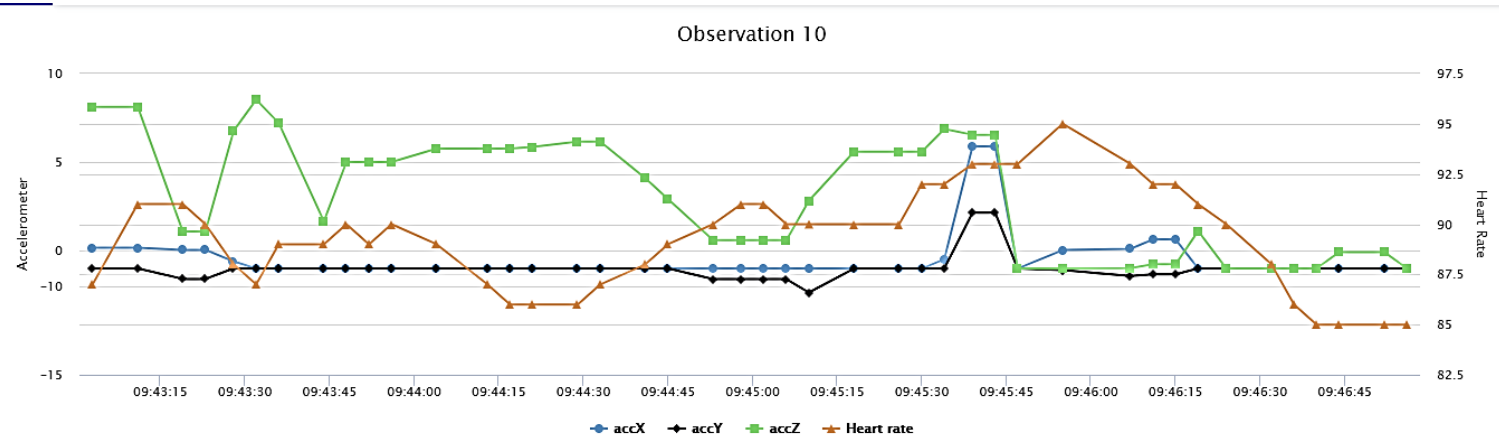

Observation 11: Seizure occurs with sensor positioned on right wrist

Observation Time: $20.35 .-20-37 \mathrm{pm}$

Seizure Occurrence: $20.36 \mathrm{pm}$

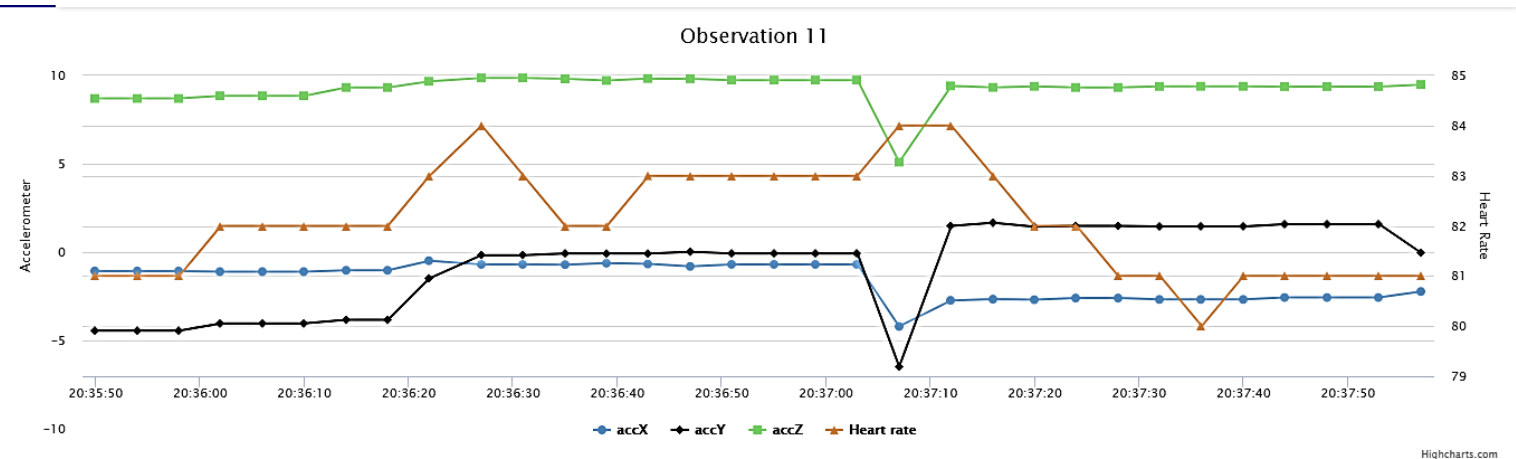

Observation 12: Seizure occurs with sensor positioned on right wrist

Observation Time: $21.05 .-21-07 \mathrm{pm}$

Seizure Occurrence: $21.06 \mathrm{pm}$

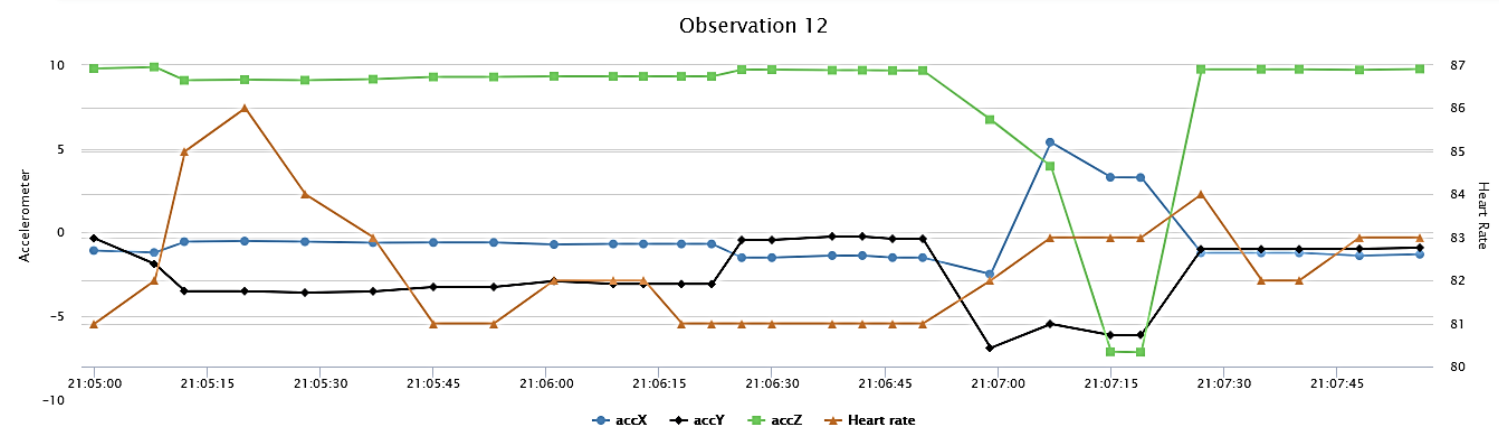


Observation a HP1: Seizure occurs with sensor positioned on right wrist

Observation Time: 09.54.-09.56am

Seizure Occurrence: 09.55am

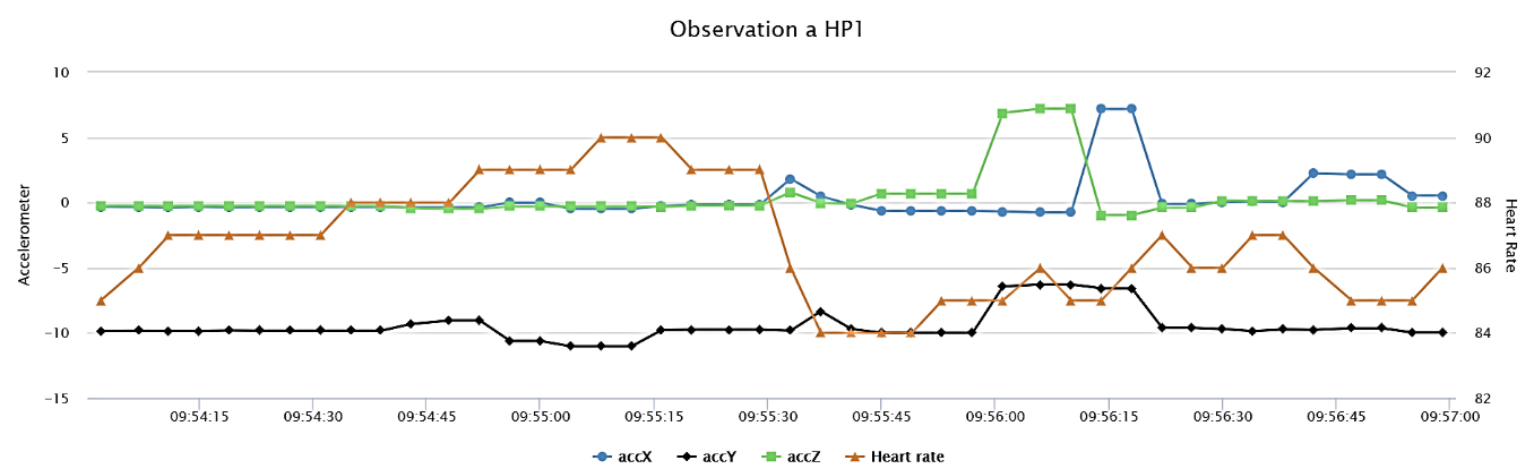

Observation b HP1: Seizure occurs with sensor positioned on right wrist

Observation Time: 10.01.-10.05am

Seizure Occurrence: 10.02am

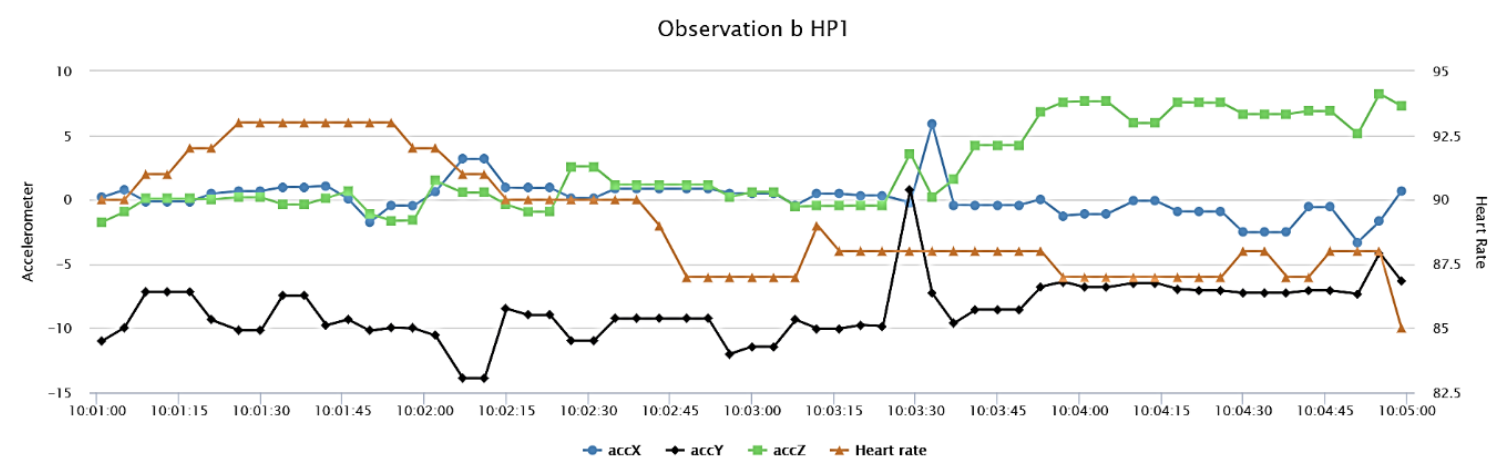

Observation c HP1: Seizure occurs with sensor positioned on right wrist

Observation Time: $19.25-19.29 \mathrm{pm}$

Seizure Occurrence: $19.26 \mathrm{pm}$

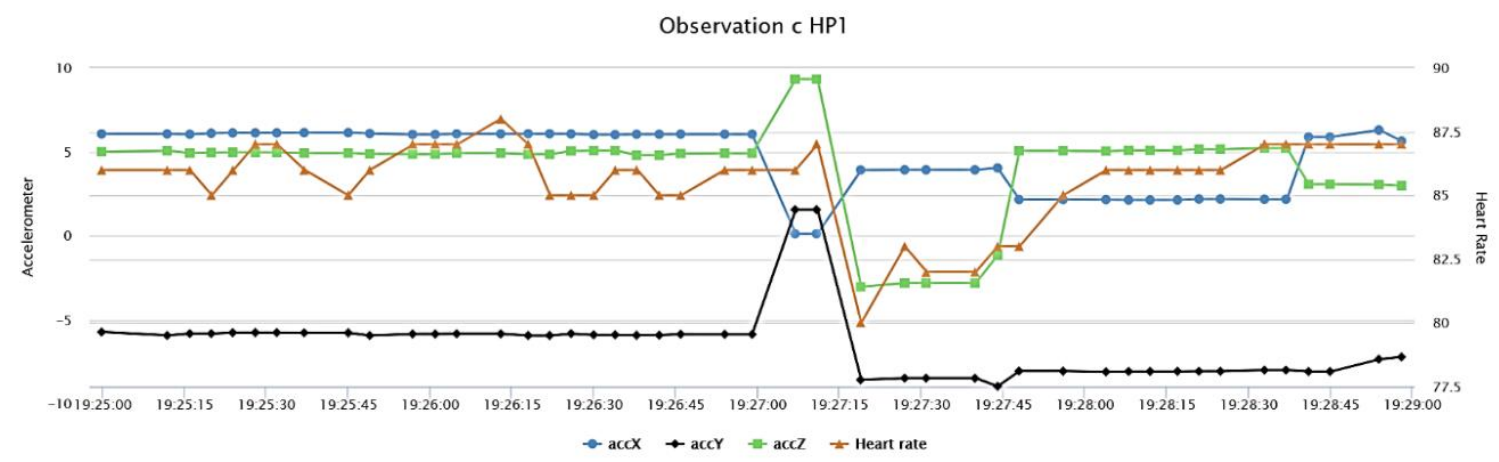


Observation d HP1: Seizure occurs with sensor positioned on right wrist

Observation Time: 20.15-20:19pm

Seizure Occurrence: $20.17 \mathrm{pm}$

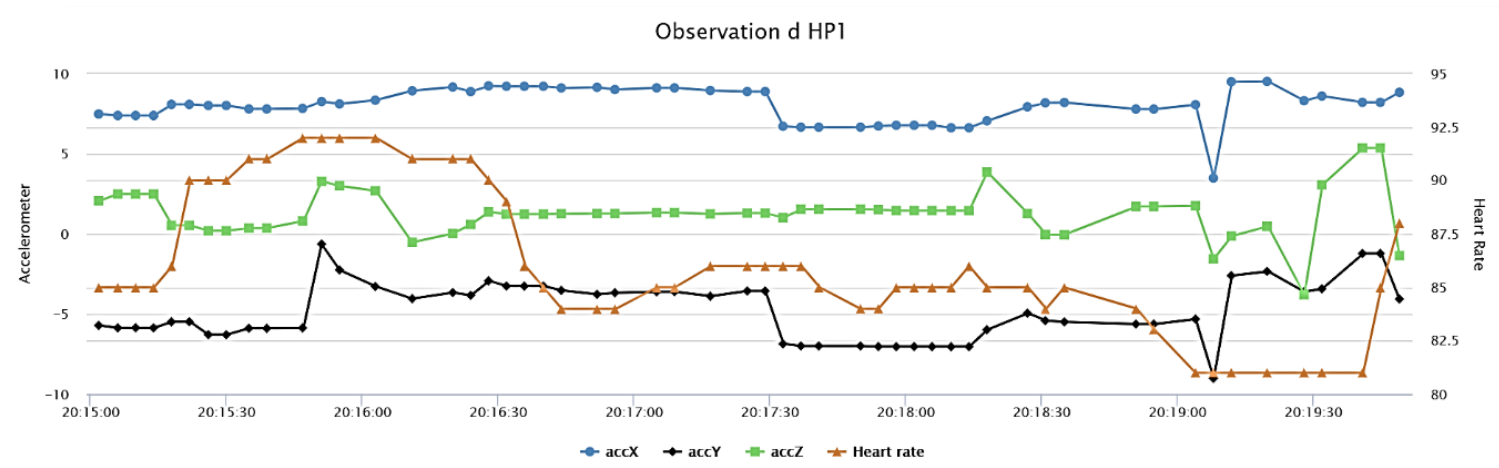

Observation e HP1: Seizure occurs with sensor positioned on right wrist

Observation Time: $20.58-21.03 \mathrm{pm}$

Seizure Occurrence: $21.00 \mathrm{pm}$

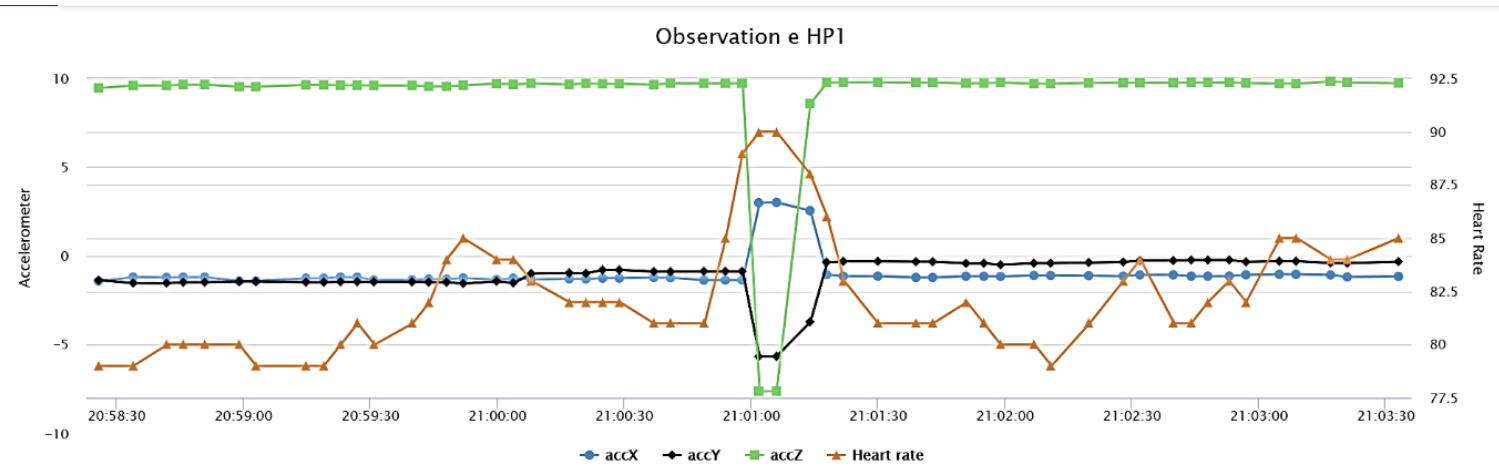

Observation f HP1: Seizure occurs with sensor positioned on right wrist

Observation Time: 10.59-11.04am

Seizure Occurrence: $11.01 \mathrm{am}$

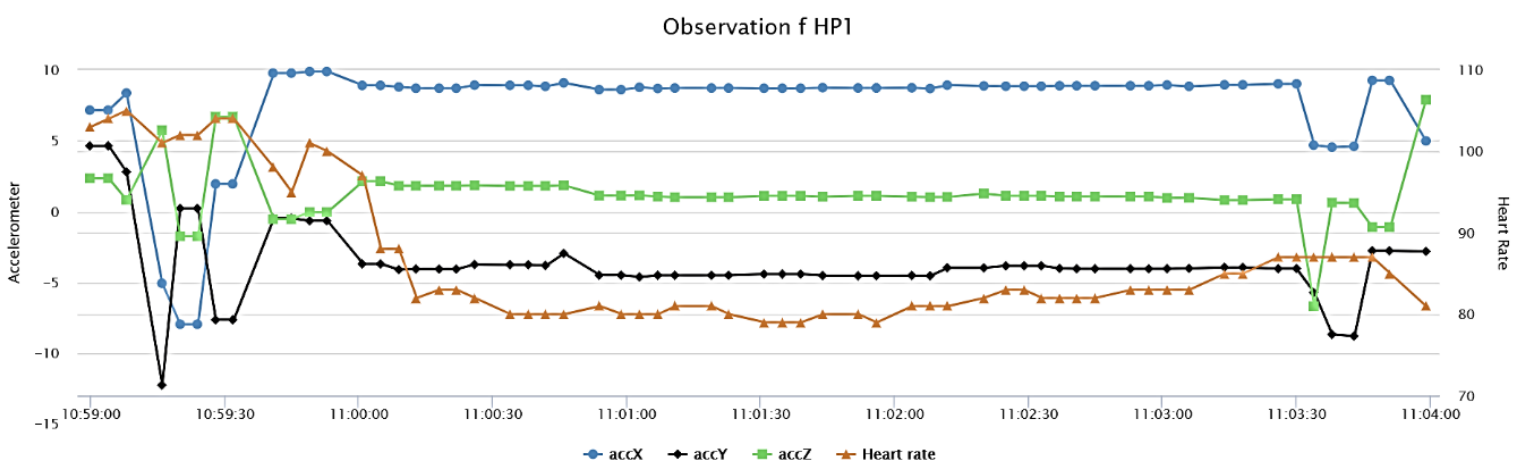


Observation g HP1: Seizure occurs with sensor positioned on right wrist

Observation Time: $20.42-20.47 \mathrm{pm}$

Seizure Occurrence: $20.44 \mathrm{pm}$

Observation g HPI

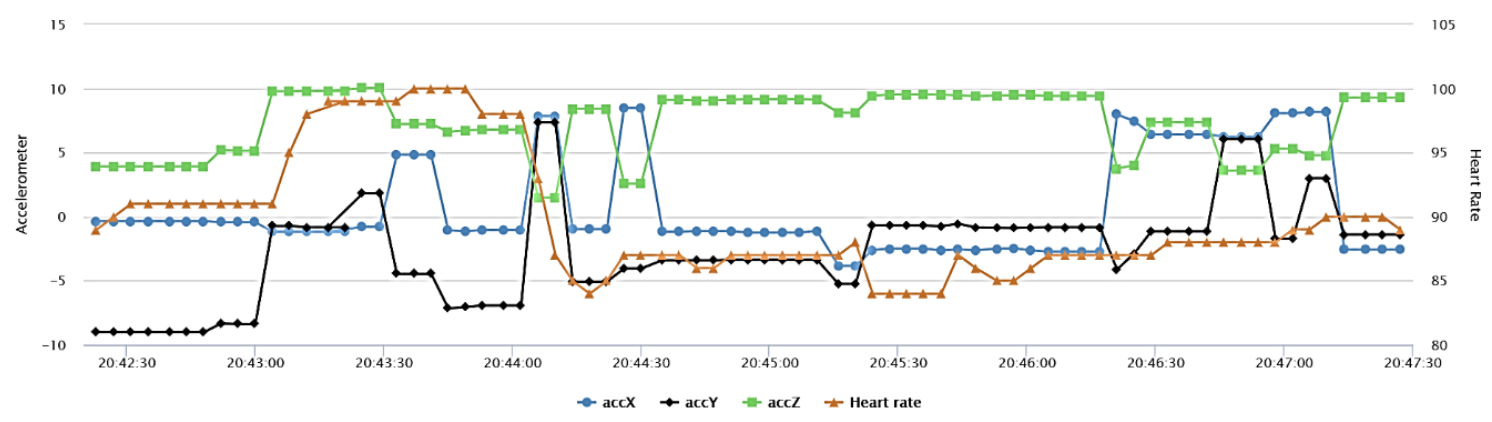

Observation h HP1: Seizure occurs with sensor positioned on right wrist

Observation Time: $20.48-20.52 \mathrm{pm}$

Seizure Occurrence: $20.50 \mathrm{pm}$

Observation h HPl

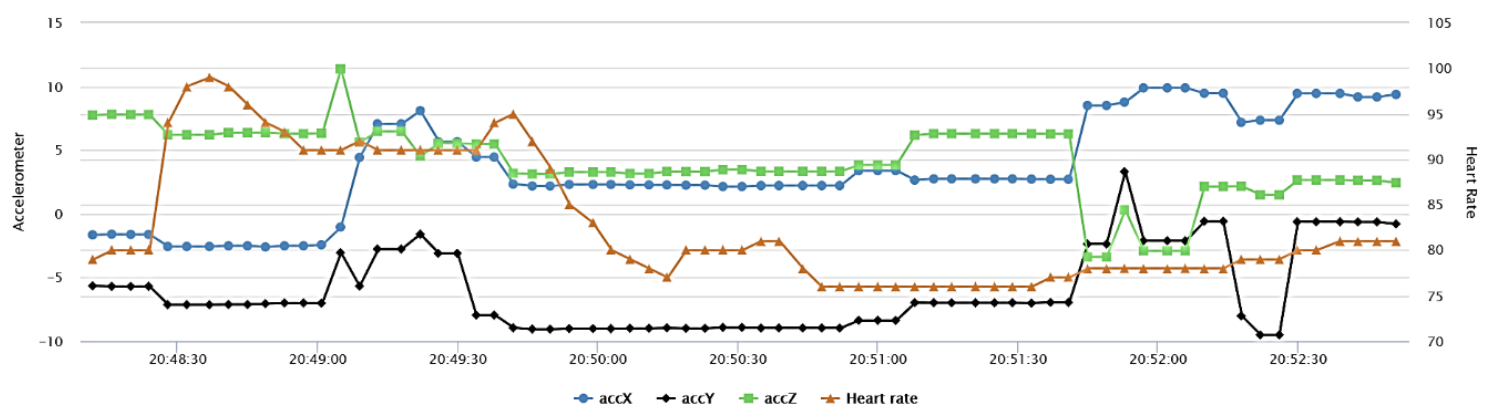

Observation 13: No seizure occurs with sensor positioned on right wrist during day-time

Observation Time: $12.52-12.54 \mathrm{pm}$

Seizure Occurrence: No seizure

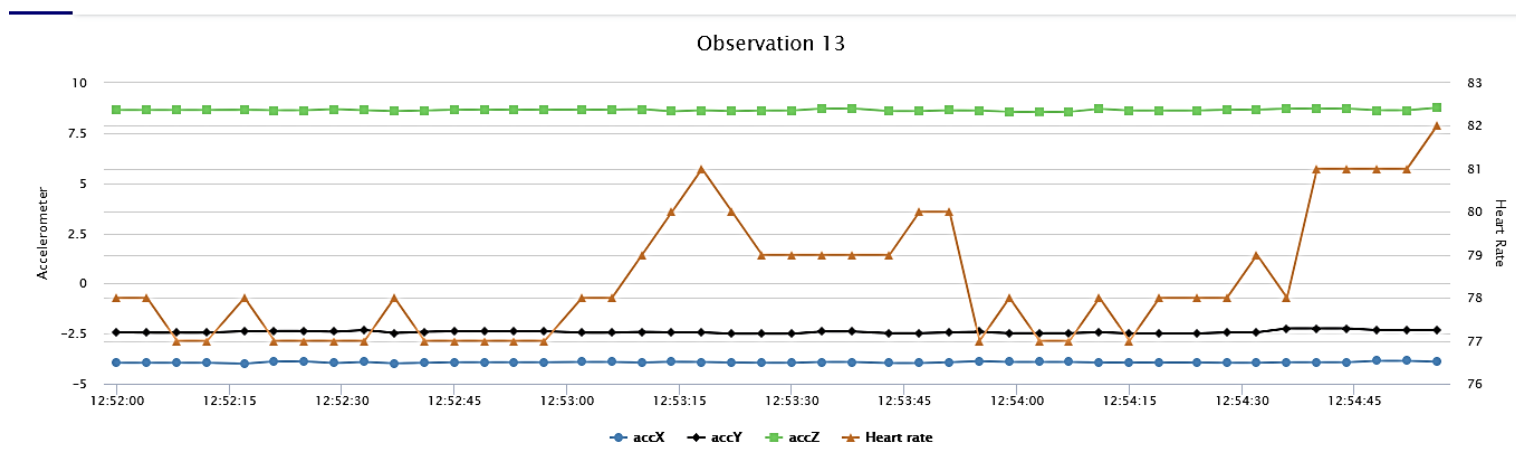


Observation 14: No seizure occurs with sensor positioned on right wrist during sleep

Observation Time: $04.50-04.52 \mathrm{am}$

Seizure Occurrence: No seizure

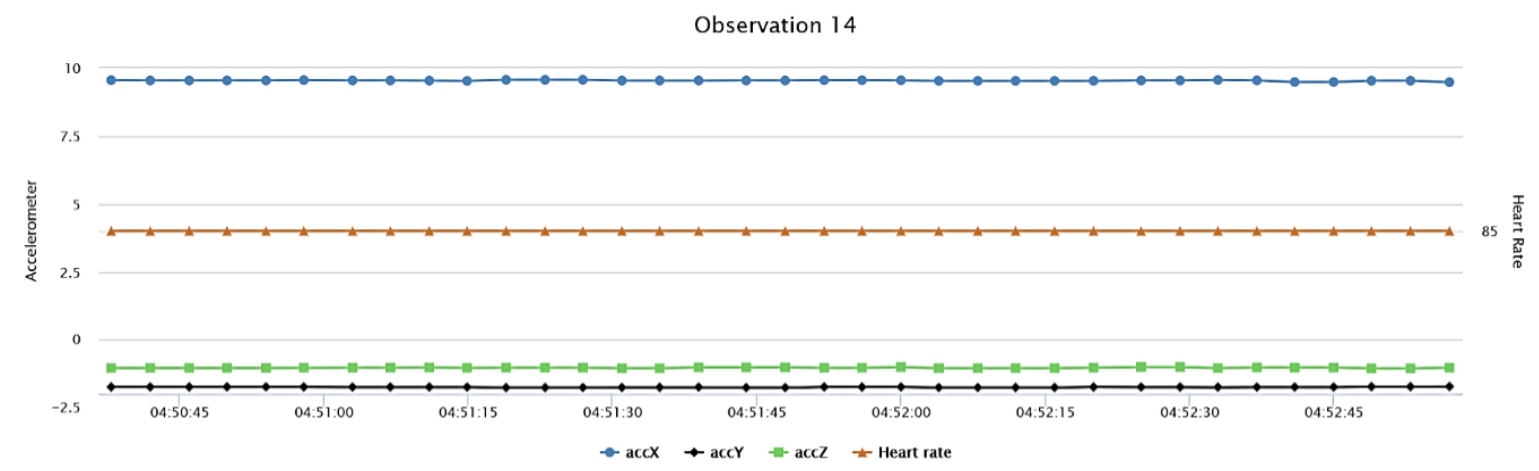

Observation 15: No seizure occurs with sensor positioned on right wrist Observation

Time: $19.03-19.06 \mathrm{pm}$

Seizure Occurrence: No seizure

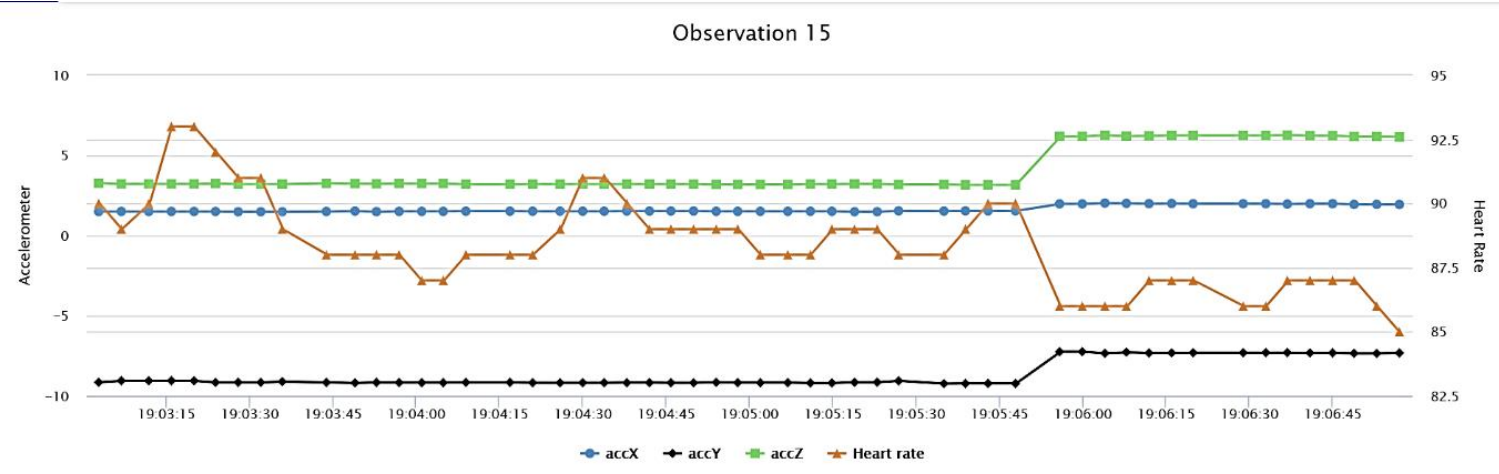

Observation 16: No seizure occurs with sensor positioned on right wrist Observation

Time: $19.18-19.22 \mathrm{pm}$

Seizure Occurrence: No seizure

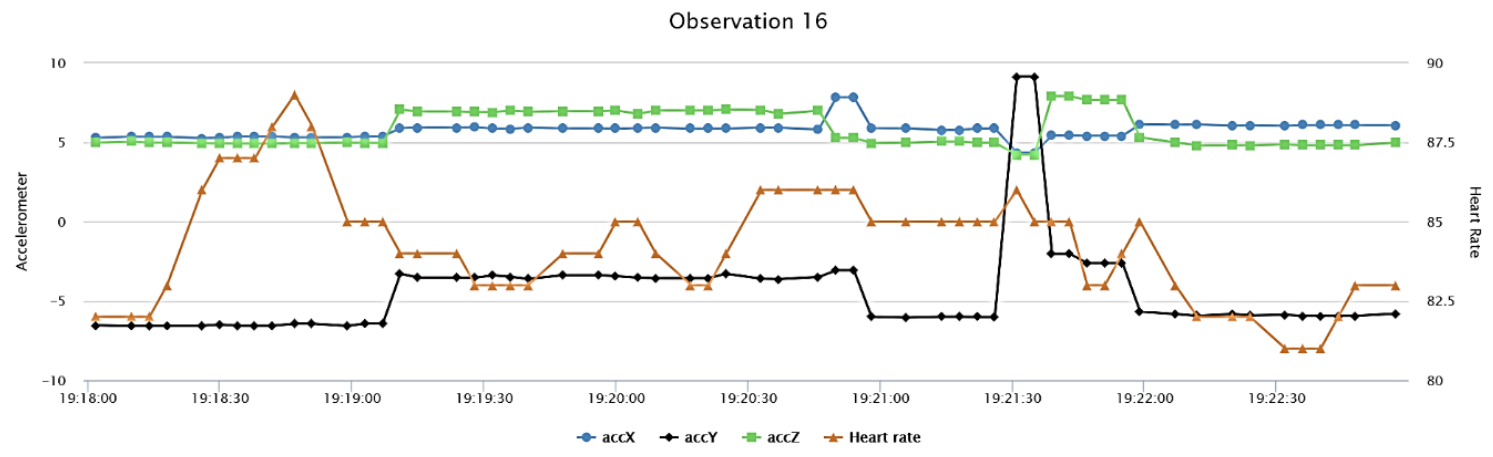


Observation 17: No seizure occurs with sensor positioned on right wrist Observation

Time: 19.36 -19.40pm

Seizure Occurrence: No seizure

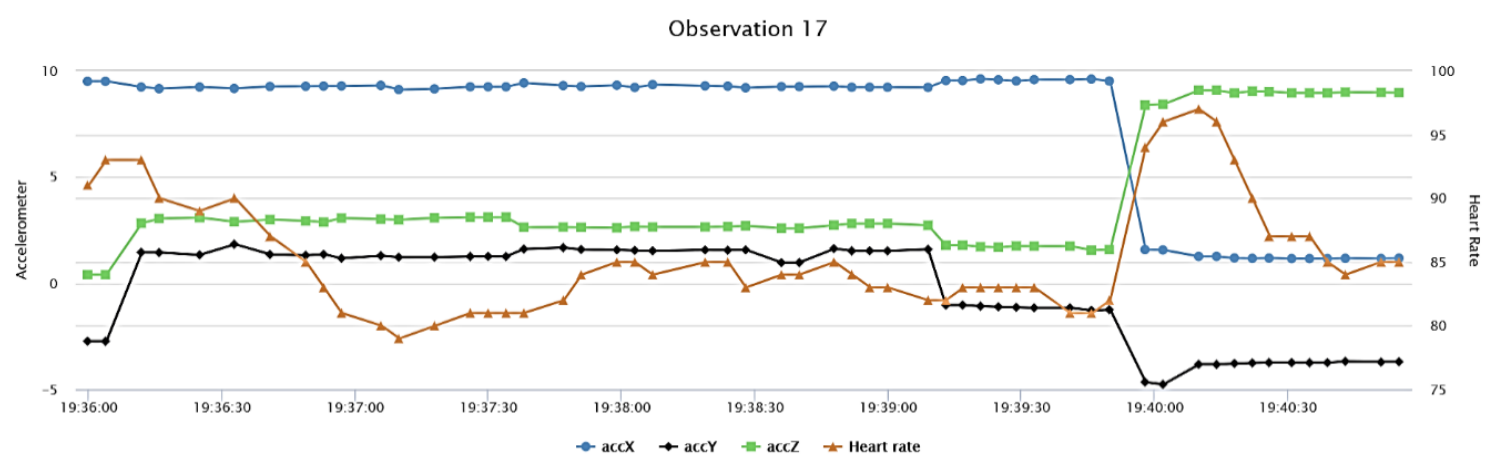




\section{Hospital Patient 2 Information}

\begin{tabular}{|l|l|}
\hline Patient Profile: & HP2 \\
\hline Seizure Type: & $\begin{array}{l}\text { FSIA (Focal seizures with impaired of } \\
\text { awareness.) } \\
\text { (main seizure type) }\end{array}$ \\
\hline Dominant Side: & Left and Right \\
\hline Time of Day: & Diurnal \\
\hline Trigger: & Unknown \\
\hline Key Symptoms: & LOC (Loss of Consciousness), Clonus, Automatisms \\
\hline
\end{tabular}

\section{Observation Criteria}

\begin{tabular}{|l|l|}
\hline Observation & Criteria \\
\hline Observation a HP2 & $\begin{array}{l}\text { No seizure occurs with sensor positioned on left wrist } \\
\text { (during sleep) }\end{array}$ \\
\hline Observation b HP2 & $\begin{array}{l}\text { No seizure occurs with sensor positioned on left wrist } \\
\text { (during day-time) }\end{array}$ \\
\hline Observation c HP2 & $\begin{array}{l}\text { No seizure occurs with sensor positioned on left wrist } \\
\text { (during day-time) }\end{array}$ \\
\hline Observation d HP2 & $\begin{array}{l}\text { No seizure occurs with sensor positioned on left wrist } \\
\text { (during evening) }\end{array}$ \\
\hline Observation e HP2 & $\begin{array}{l}\text { No seizure occurs with sensor positioned on left wrist } \\
\text { (during afternoon) }\end{array}$ \\
\hline
\end{tabular}

\section{Results}

Observation a HP2: No seizure occurs with sensor positioned on left wrist during sleep Observation Time: $06.27-06.30 \mathrm{am}$

Seizure Occurrence: No seizure

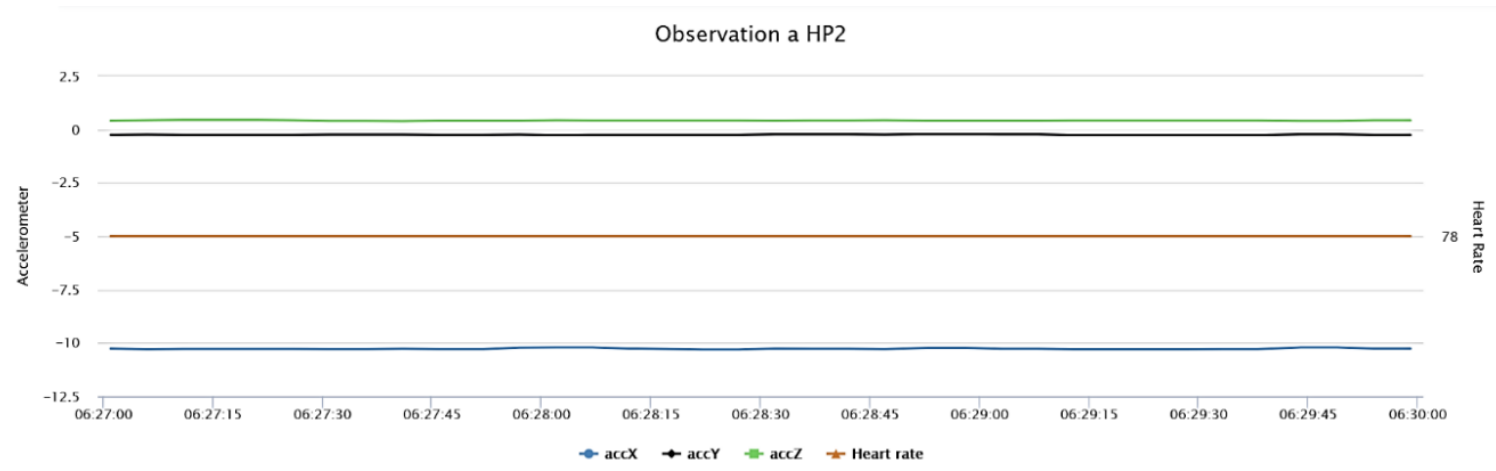


Observation b HP2: No seizure occurs with sensor positioned on left wrist during day-time Observation Time: 10.54.-10-56am

Seizure Occurrence: No seizure

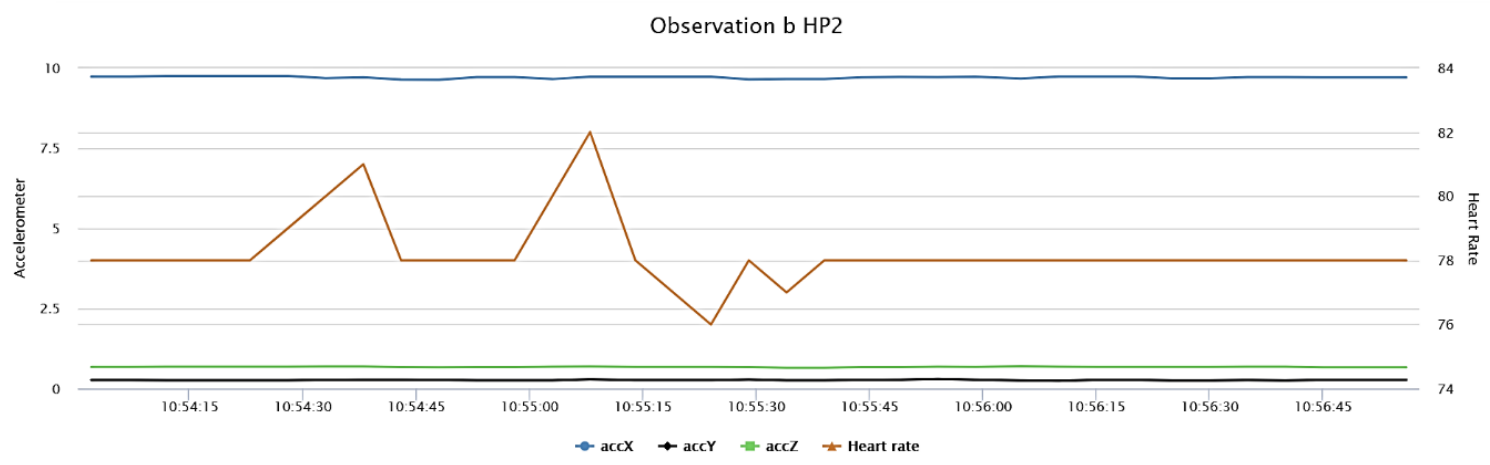

Observation c HP2: No seizure occurs with sensor positioned on left wrist during day-time Observation Time: $15.05-15.07 \mathrm{pm}$

Seizure Occurrence: No seizure

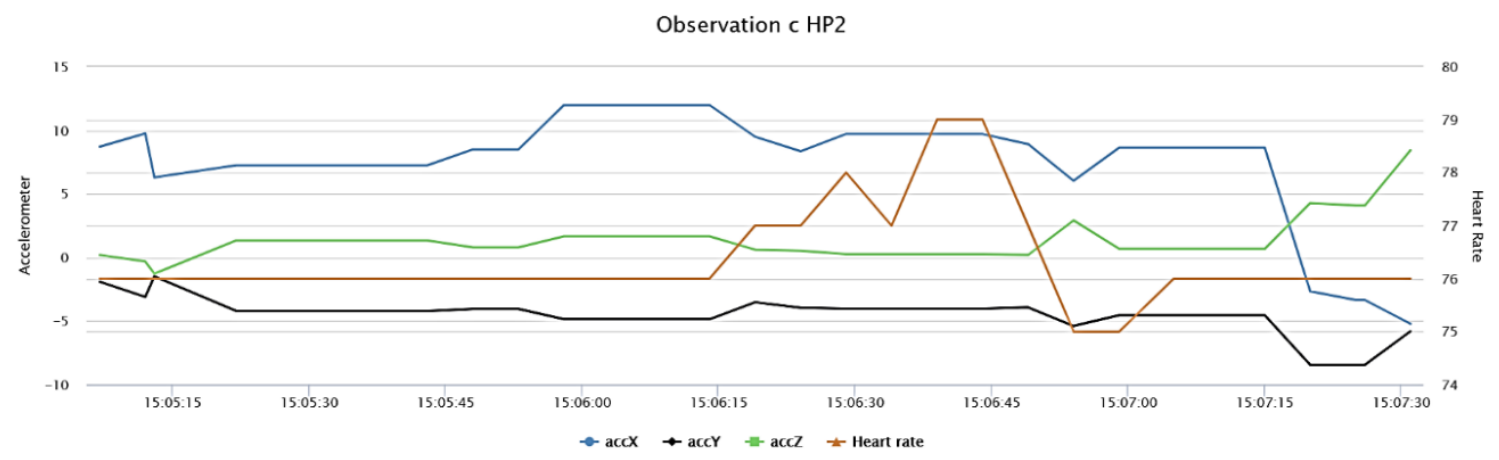

Observation d HP2: No seizure occurs with sensor positioned on left wrist during day-time Observation Time: $19.39-19.41 \mathrm{pm}$

Seizure Occurrence: No seizure

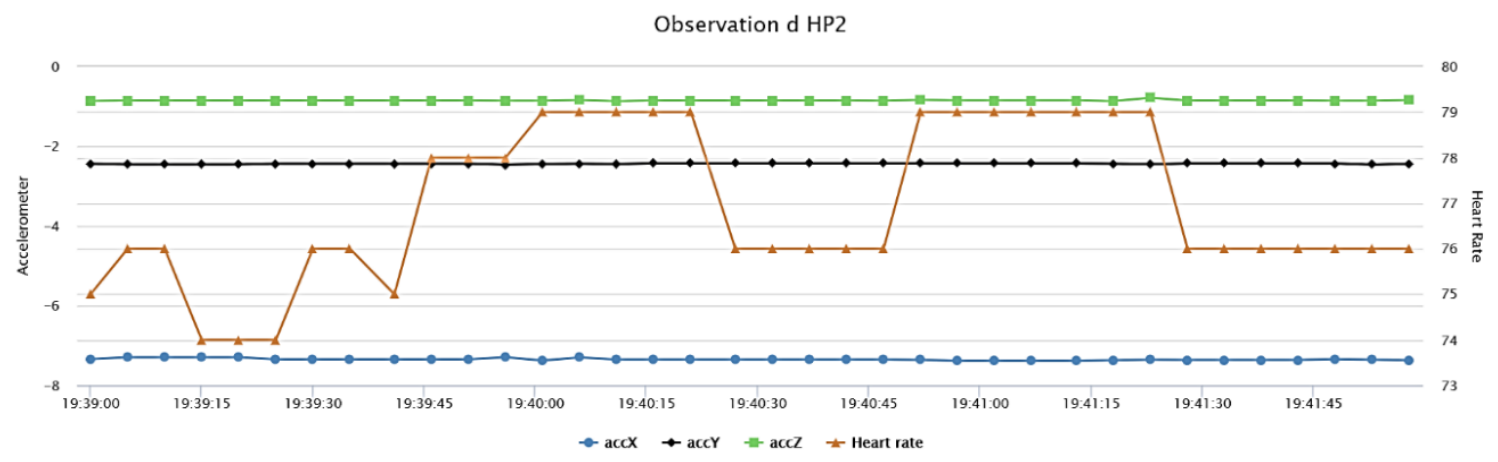


Observation e HP2: No seizure occurs with sensor positioned on left wrist during day-time Observation Time: $13.54-13.57 \mathrm{pm}$

Seizure Occurrence: No seizure

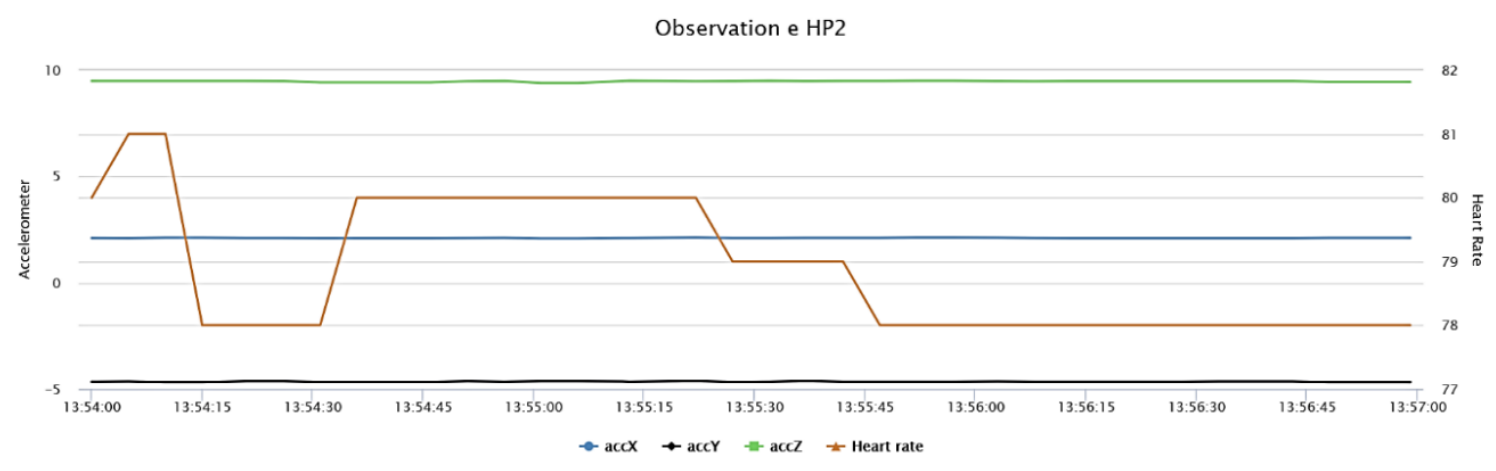


Hospital Patient 3 Information

\begin{tabular}{|l|l|}
\hline Patient Profile: & HP3 \\
\hline Seizure Type: & $\bullet$\begin{tabular}{l|} 
GTCS. \\
\end{tabular} \\
& $\begin{array}{l}\text { FSIA (Focal seizures with impaired of } \\
\text { awarens.) }\end{array}$ \\
\hline Dominant Side: & Left and Right \\
\hline Time of Day: & Diurnal and Nocturnal \\
\hline Trigger: & Unknown \\
\hline Key Symptoms: & Full Body Shaking (Clonus), LOC,Automatisms \\
\hline
\end{tabular}

\section{Observation Criteria}

\begin{tabular}{|l|l|}
\hline Observation & Criteria \\
\hline Observation a HP3 & $\begin{array}{l}\text { No seizure occurs with sensor positioned on right wrist } \\
\text { (during day-time) }\end{array}$ \\
\hline Observation b HP3 & $\begin{array}{l}\text { No seizure occurs with sensor positioned on right wrist } \\
\text { (during sleep) }\end{array}$ \\
\hline Observation c HP3 & $\begin{array}{l}\text { No seizure occurs with sensor positioned on right wrist } \\
\text { (during day-time) }\end{array}$ \\
\hline Observation e HP3 & $\begin{array}{l}\text { No seizure occurs with sensor positioned on right wrist } \\
\text { (during day-time, } \text { evening) }\end{array}$ \\
\hline
\end{tabular}

\section{Results}

Observation a HP3: No seizure occurs with sensor positioned on right wrist during day-time Observation Time: 10.10-10.12am

Seizure Occurrence: No seizure

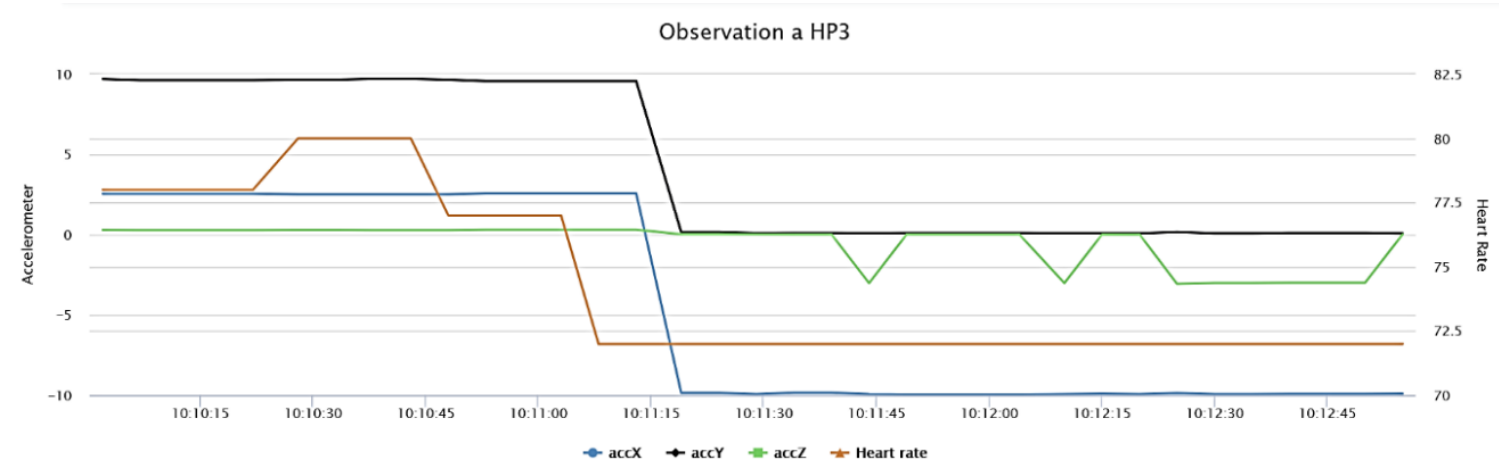


Observation b HP3: No seizure occurs with sensor positioned on right wrist during sleep Observation Time: 05.01 - 05.03am

Seizure Occurrence: No seizure

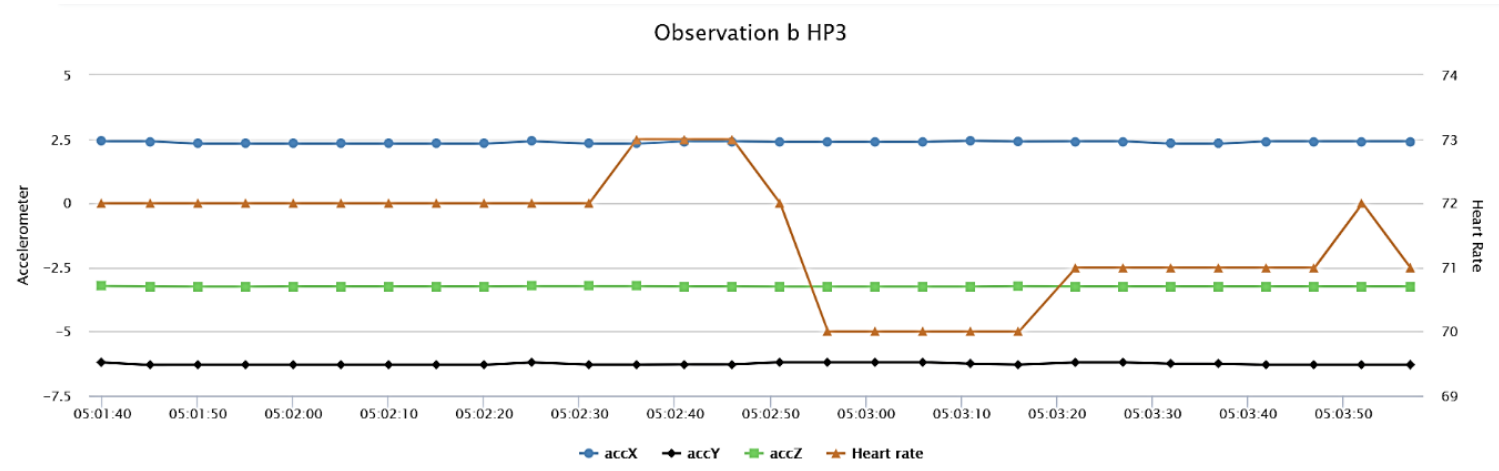

Observation c HP3: No seizure occurs with sensor positioned on right wrist during day-time Observation Time: 08.09am - 08.12am

Seizure Occurrence: No seizure

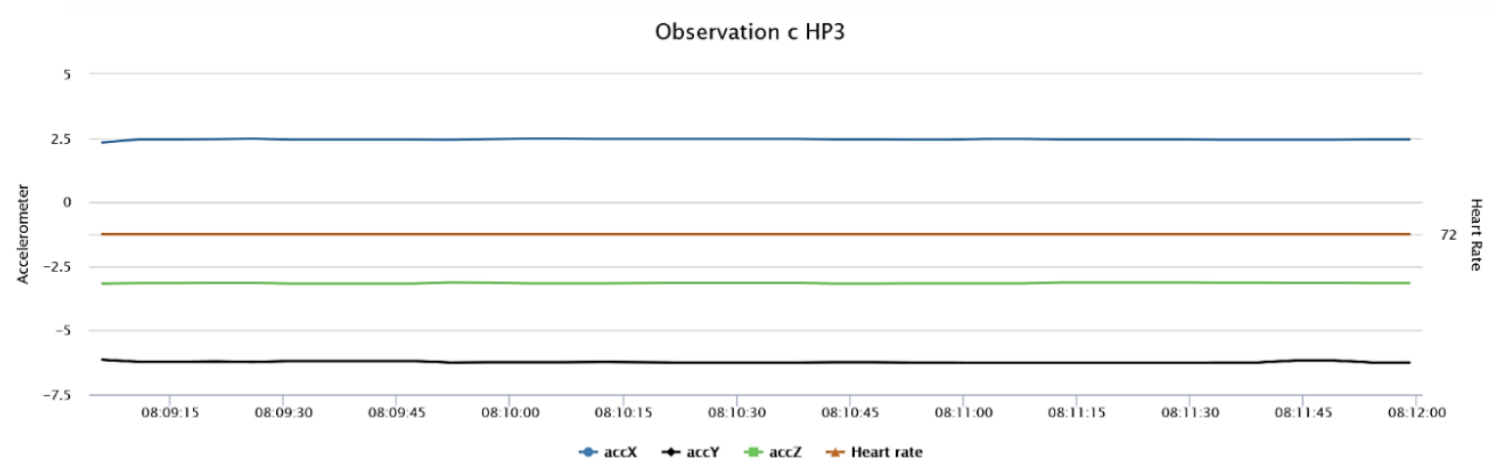

Observation d HP3: No seizure occurs with sensor positioned on right wrist during daytime

Observation Time: 13.40- 13.43pm

Seizure Occurrence: No seizure

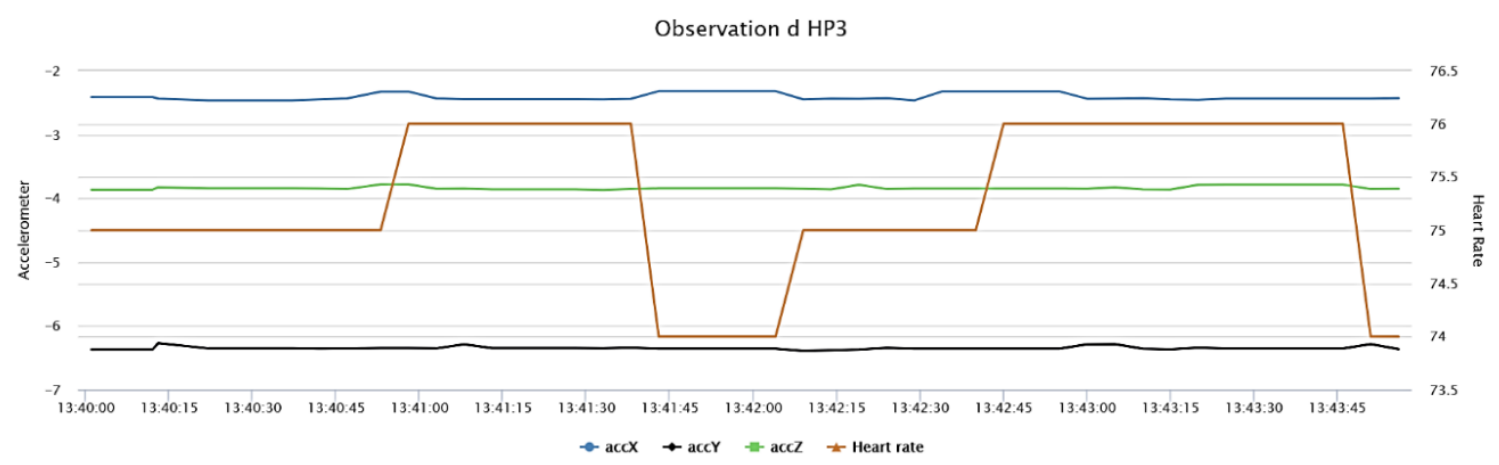


Observation e HP3: No seizure occurs with sensor positioned on right wrist during day-time Observation Time: 19.13 -19.17am

Seizure Occurrence: No seizure

Observation e HP3

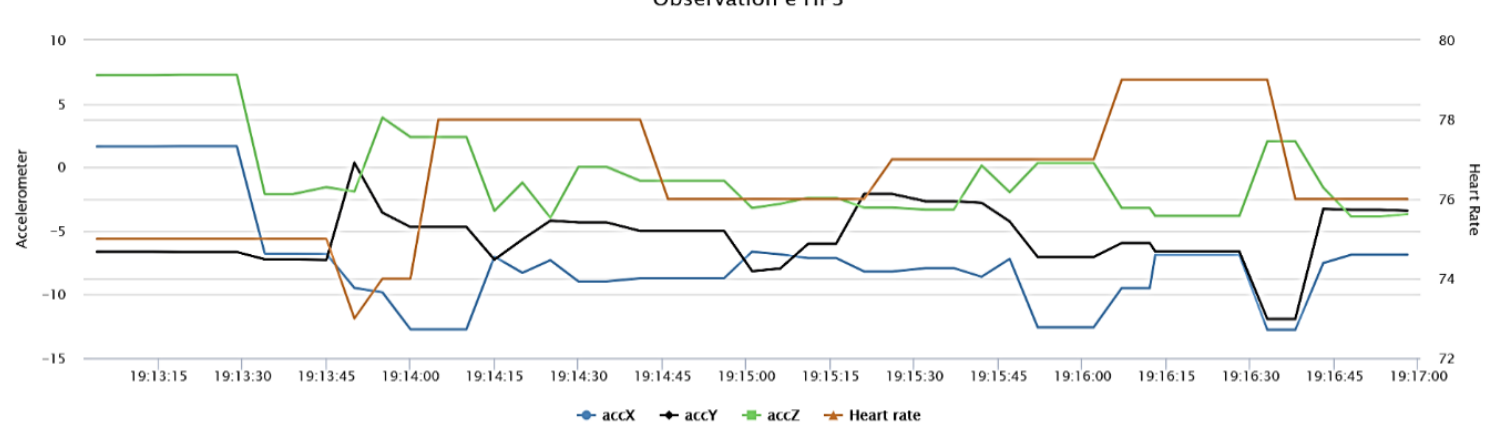


$>$ Hospital Patient 4 Information

\begin{tabular}{|l|l|}
\hline Patient Profile: & HP4 \\
\hline Seizure Type: & $\begin{array}{l}\bullet \text { GTCS (Generalised Tonic Clonic Seizure) } \\
\text { awareness.) }\end{array}$ \\
\hline Dominant Side: & Right Arm \\
\hline Time of Day: & Nocturnal (all from sleep) \\
\hline Trigger: & Unknown \\
\hline Key Symptoms: & $\begin{array}{l}\text { LOC, Full Body Shaking (Clonus), and behavioural } \\
\text { change (Automatisms) }\end{array}$ \\
\hline
\end{tabular}

\section{Observation Criteria}

\begin{tabular}{|c|c|}
\hline Observation & Criteria \\
\hline Observation a HP4 & $\begin{array}{l}\text { Seizure occurs with sensor positioned on right wrist (from } \\
\text { sleep) }\end{array}$ \\
\hline Observation b HP4 & $\begin{array}{l}\text { Seizure occurs with sensor positioned on right wrist (from } \\
\text { sleep) }\end{array}$ \\
\hline Observation c HP4 & $\begin{array}{l}\text { Seizure occurs with sensor positioned on right wrist (from } \\
\text { sleep) }\end{array}$ \\
\hline Observation d HP4 & $\begin{array}{l}\text { Seizure occurs with sensor positioned on right wrist (from } \\
\text { sleep) }\end{array}$ \\
\hline Observation e HP4 & $\begin{array}{l}\text { Seizure occurs with sensor positioned on right wrist (from } \\
\text { sleep) }\end{array}$ \\
\hline Observation f HP4 & $\begin{array}{l}\text { Seizure occurs with sensor positioned on right wrist (from } \\
\text { sleep) }\end{array}$ \\
\hline Observation g HP4 & $\begin{array}{l}\text { No seizure occurs with sensor positioned on right wrist } \\
\text { (during day-time) }\end{array}$ \\
\hline Observation h HP4 & $\begin{array}{l}\text { No seizure occurs with sensor positioned on right wrist } \\
\text { (during day-time) }\end{array}$ \\
\hline Observation i HP4 & $\begin{array}{l}\text { No seizure occurs with sensor positioned on right wrist } \\
\text { (during day-time) }\end{array}$ \\
\hline Observation j HP4 & $\begin{array}{l}\text { No seizure occurs with sensor positioned on right wrist } \\
\text { (during day-time) }\end{array}$ \\
\hline Observation k HP4 & $\begin{array}{l}\text { No seizure occurs with sensor positioned on right wrist } \\
\text { (during day-time) }\end{array}$ \\
\hline
\end{tabular}




\section{Results}

Observation a HP4: Seizure occurs with sensor positioned on right wrist during day-time Observation Time: $12.42-12.45 p m$

Seizure Occurrence: $12.44 \mathrm{pm}$

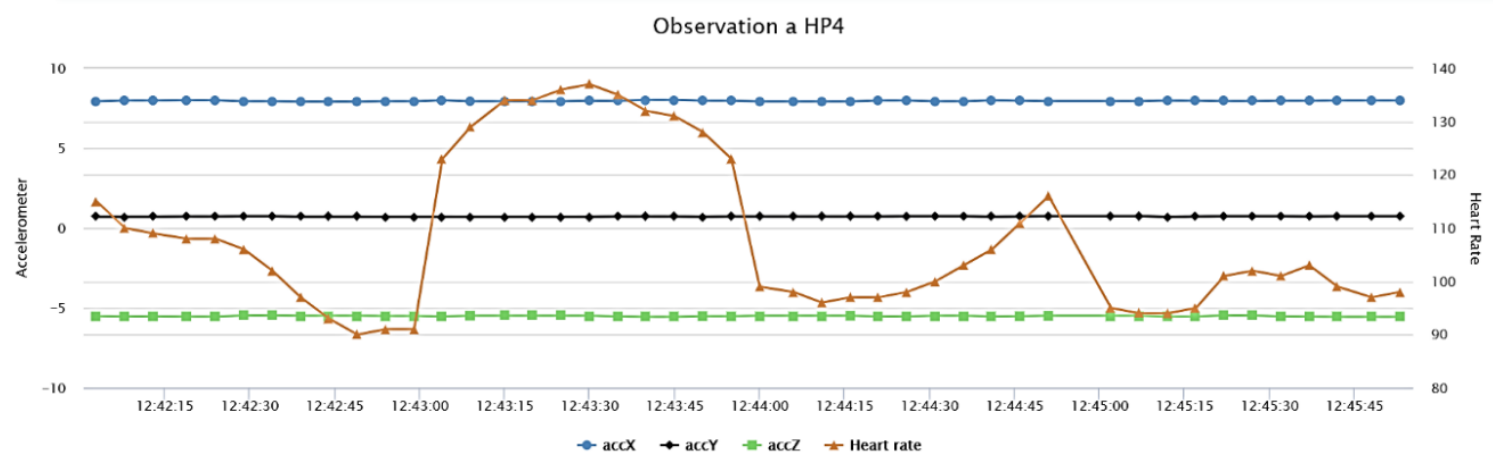

Observation b HP4: Seizure occurs with sensor positioned on right wrist

Observation Time: 00.53 -00.56am

Seizure Occurrence: 00.54am

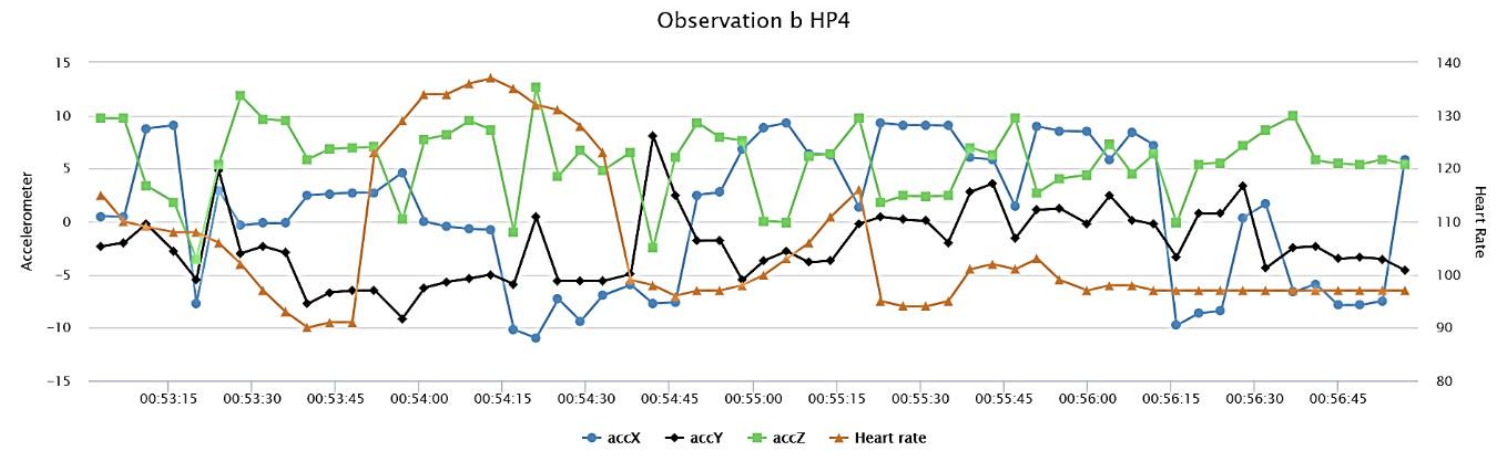

Observation c HP4: Seizure occurs with sensor positioned on right wrist

Observation Time: 00.22 -00.28am

Seizure Occurrence: 00.23am

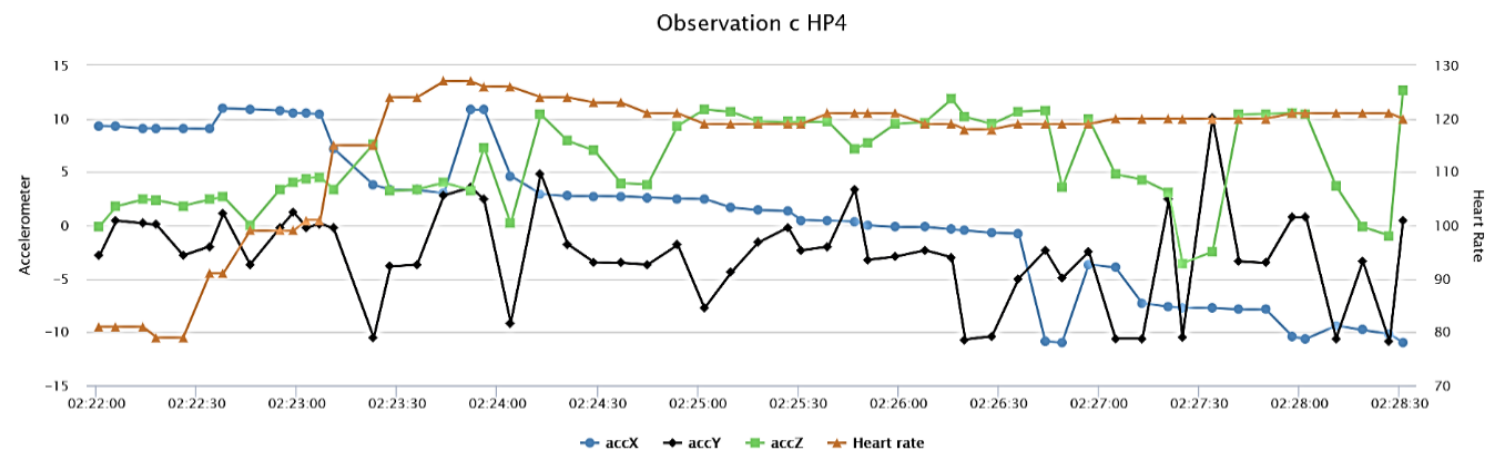


Observation d HP4: Seizure occurs with sensor positioned on right wrist

Observation Time: 04.41 -04.45am

Seizure Occurrence: 00.23am

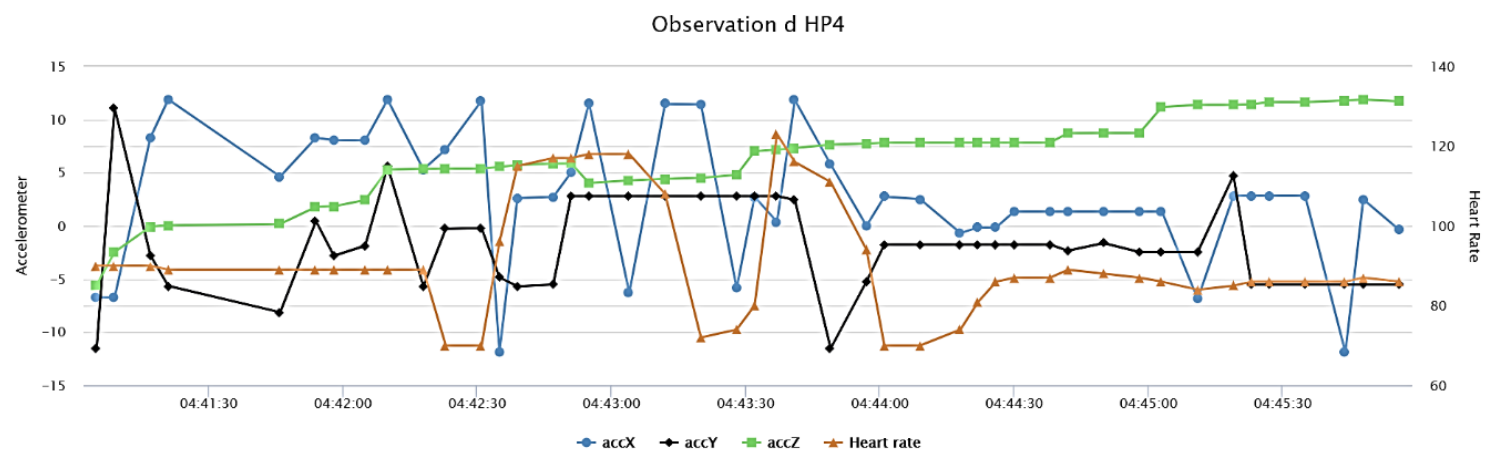

Observation e HP4: Seizure occurs with sensor positioned on right wrist

Observation Time: 06.56 -06.59am

Seizure Occurrence: $06.57 \mathrm{am}$

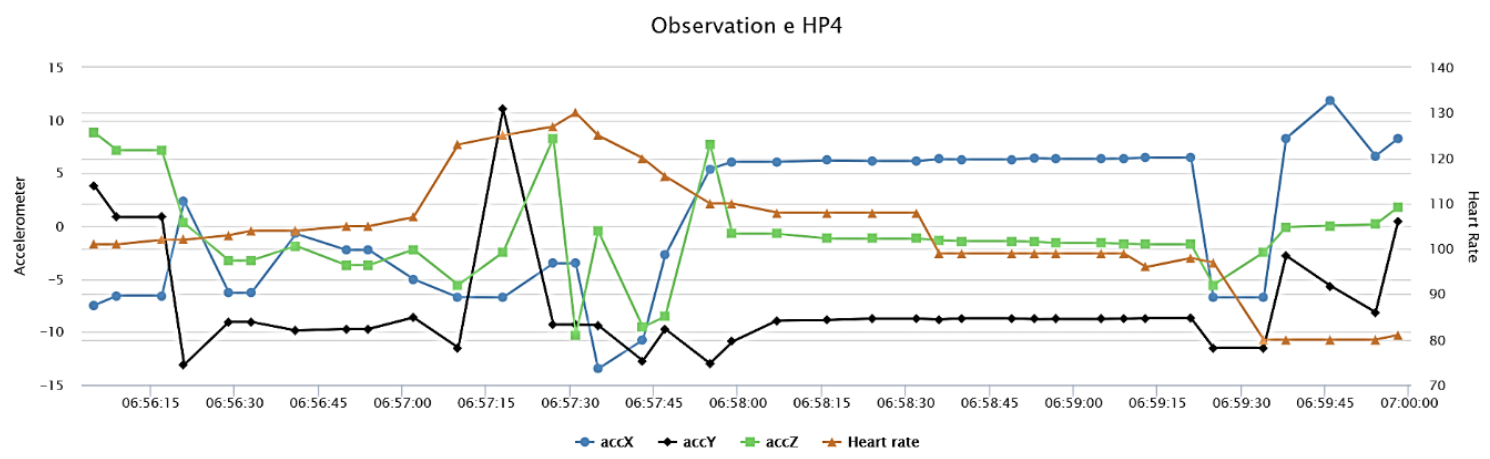

Observation f HP4: Seizure occurs with sensor positioned on right wrist

Observation Time: 10.31 -10.35am

Seizure Occurrence: 10.33am

Observation f HP4

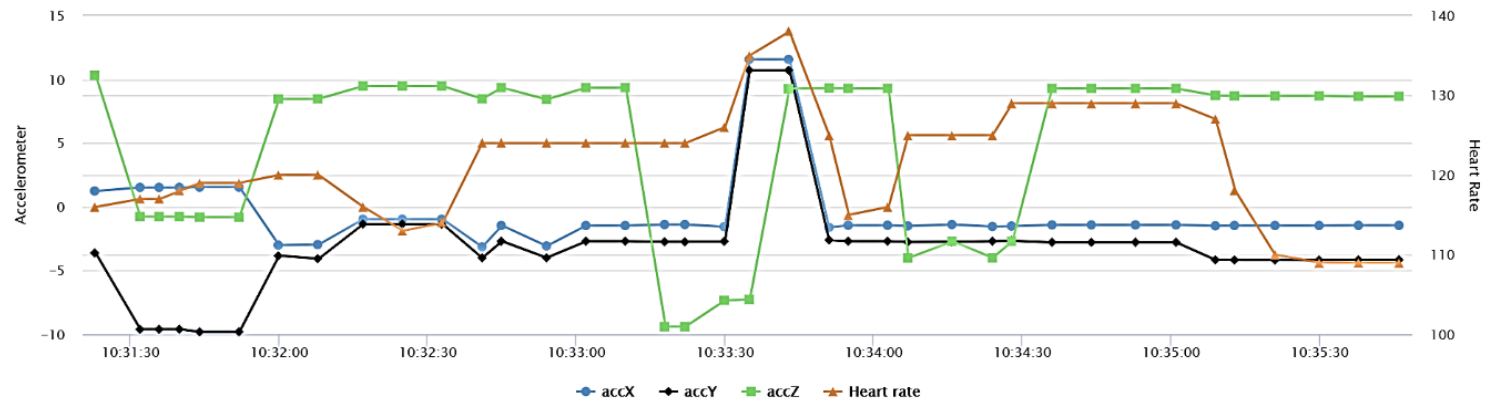


Observation g HP4: No seizure occurs with sensor positioned on right wrist

Observation Time: $13.34-13.37 p m$

Seizure Occurrence: None

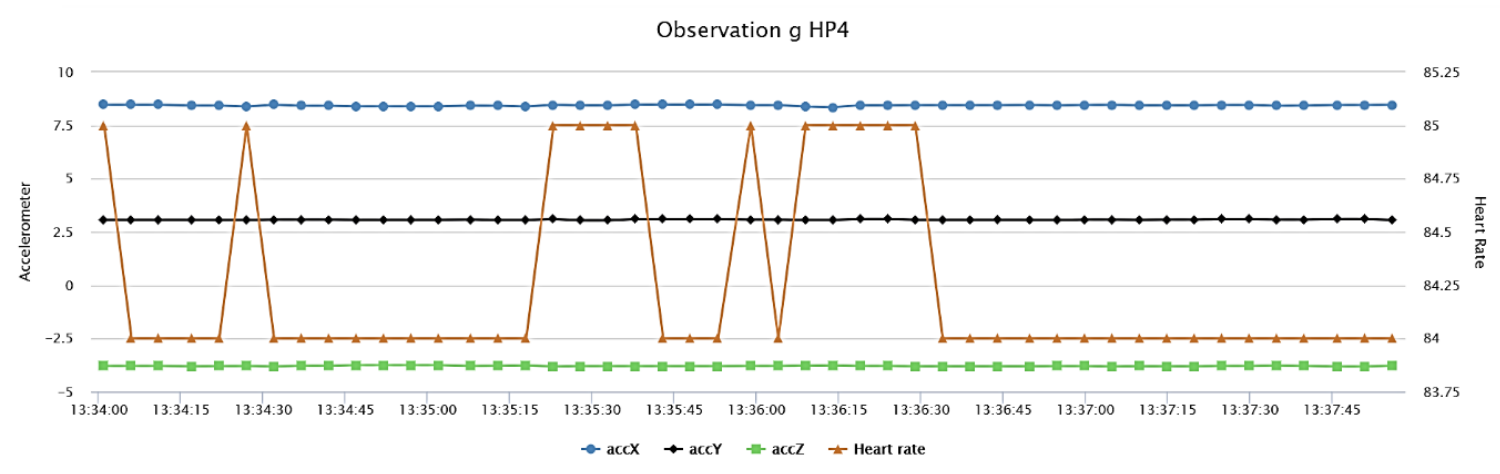

Observation h HP4: No seizure occurs with sensor positioned on right wrist Observation Time: 14.07 -14.11pm

Seizure Occurrence: None

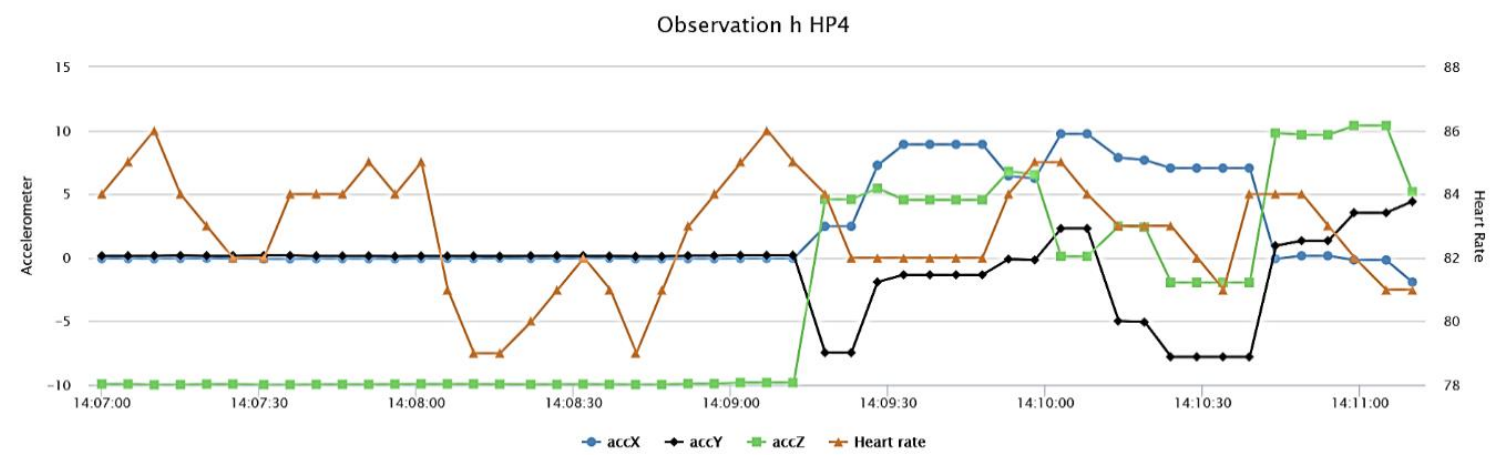

Observation i HP4: No seizure occurs with sensor positioned on right wrist

Observation Time: $08.01-08.04 \mathrm{pm}$

Seizure Occurrence: None

Observation i HP4

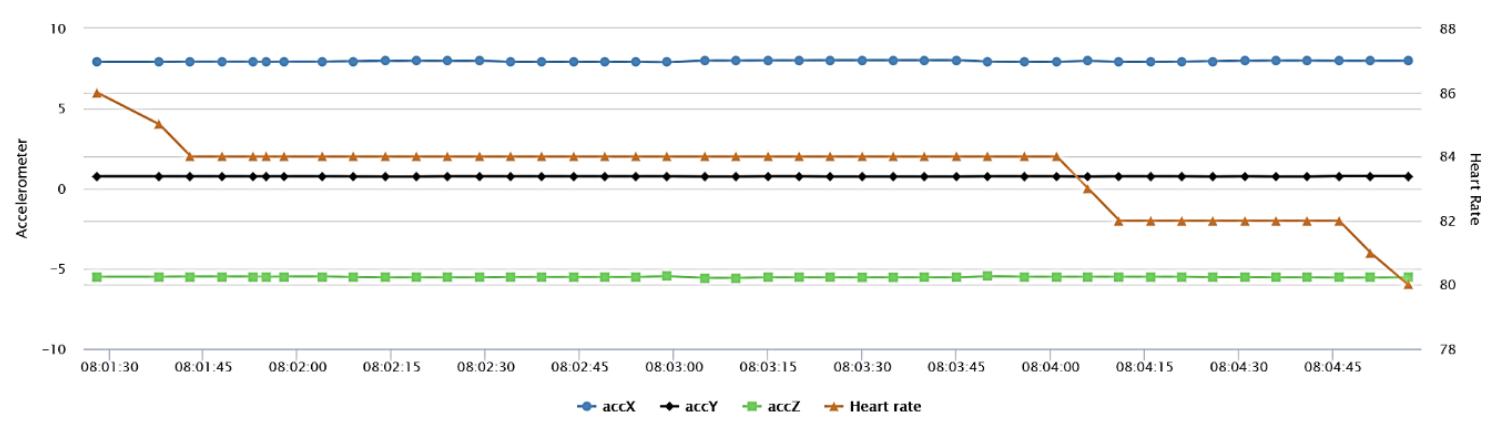


Observation j HP4: No seizure occurs with sensor positioned on right wrist

Observation Time: 09.00 -09.04pm

Seizure Occurrence: None

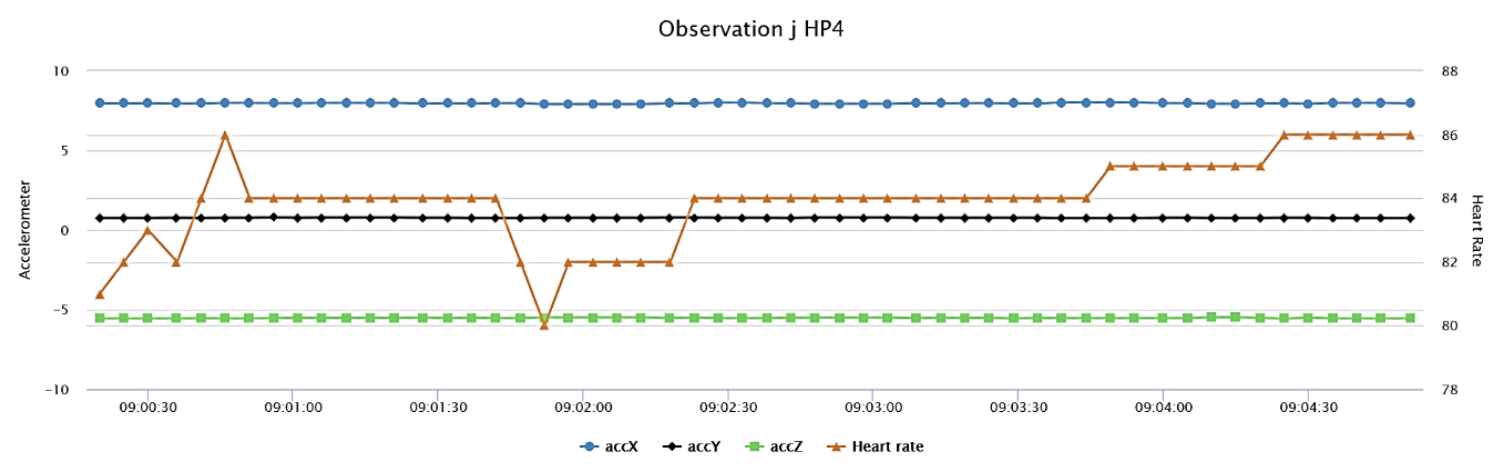

Observation k HP4: No seizure occurs with sensor positioned on right wrist Observation Time: 21.34 -21.38pm

Seizure Occurrence: None

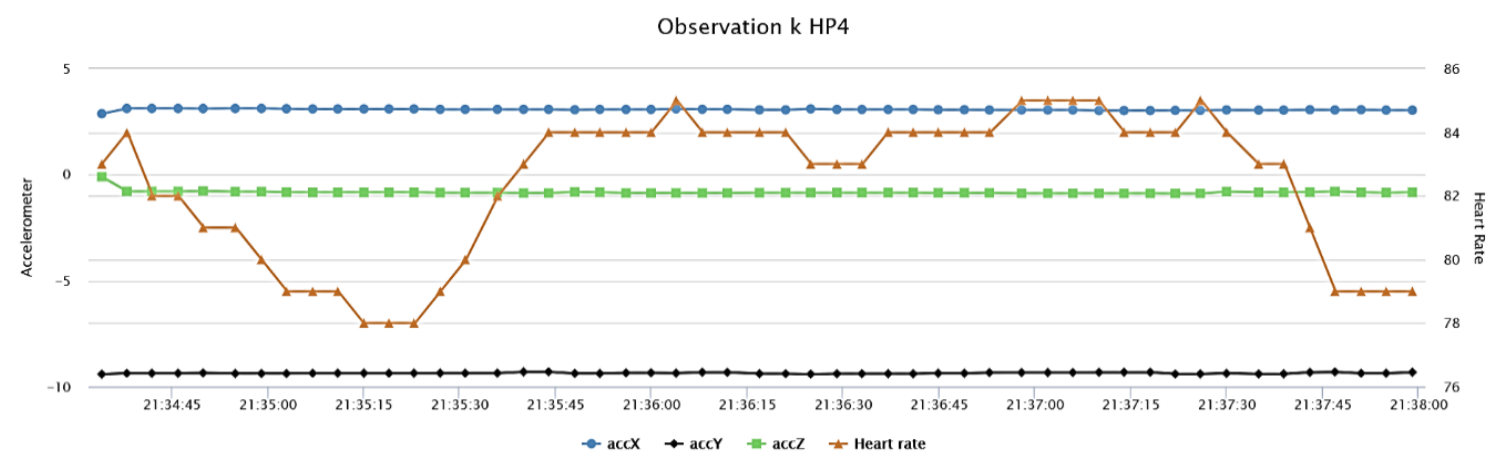


$>$ Hospital Patient 5 Information

\begin{tabular}{|l|l|}
\hline Patient Profile: & HP5 \\
\hline Seizure Type: & Unclassified \\
\hline Dominant Side: & Left \\
\hline Time of Day: & Diurnal \\
\hline Trigger: & Unknown \\
\hline Key Symptoms: & Full Body Shaking (Bilateral Clonus) \\
\hline
\end{tabular}

\section{Observation Criteria}

\section{Observation}

Observation a HP5

\section{Criteria}

One non epileptic shaking event occurs with sensor

positioned on left wrist (during day-time)

\section{Results}

Observation a HP5: Seizure occurs with sensor positioned on left wrist during evening

Observation Time: 23:01 - 23:05pm

Seizure Occurrence: 23:03pm

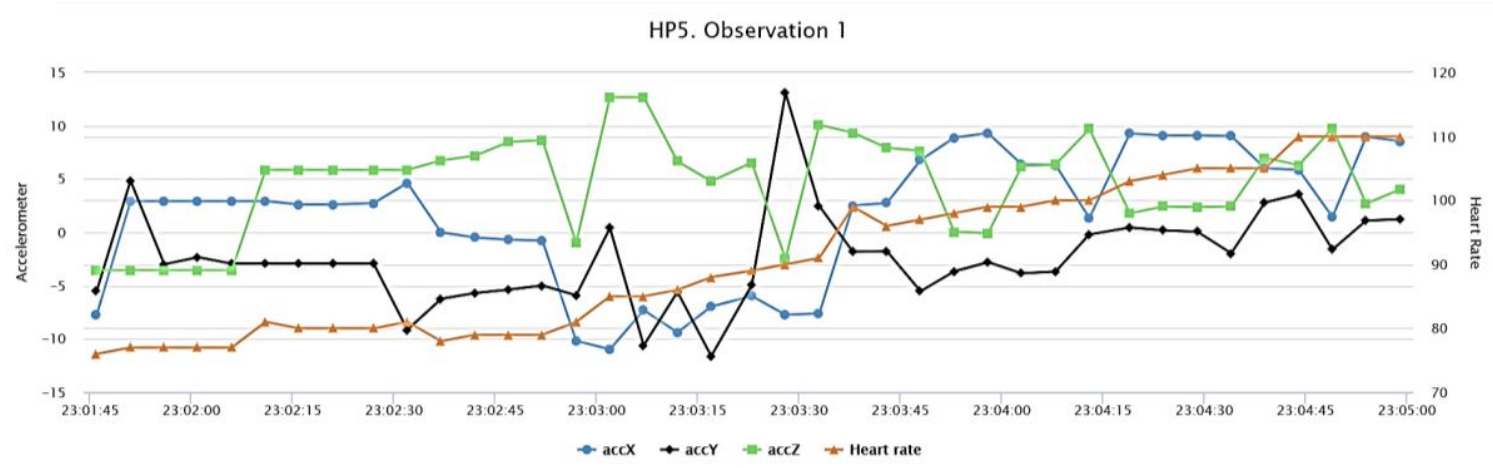




\section{Appendix C}

\section{Volunteer 1 \& 2 Observations}

\section{Volunteer 1 Information}

\begin{tabular}{|l|l|}
\hline Volunteer Profile: & V1 \\
\hline Seizure Type: & GTCS \\
\hline Dominant Side: & Right Arm \\
\hline Time of Day: & Diurnal \\
\hline Trigger: & Stress \\
\hline Key Symptoms: & $\begin{array}{l}\text { Shaking right side, right arm raised then convulsion, } \\
\text { LOC }\end{array}$ \\
\hline
\end{tabular}

\section{Observation Criteria Volunteer 1}

\begin{tabular}{|l|l|l|l|}
\hline Observation & Seizure & Position & Time of Day (Random) \\
\hline V1. Observation 1 & Yes & Right Wrist & Evening \\
\hline V1. Observation 2a & No & Right Wrist & Night Time (during sleep) \\
\hline V1. Observation 2b & No & Right Wrist & During the evening ) \\
\hline V1. Observation 2c & No & Right Wrist & During the evening \\
\hline V1. Observation 3 & Yes & Left Wrist & Day Time (Morning) \\
\hline V1. Observation 3a & No & Left Wrist & Evening \\
\hline V1. Observation 3b & No & Left Wrist & Day Time (Morning) \\
\hline V1. Observation 3c & No & Left Wrist & Evening \\
\hline V1. Observation 4 & Yes & Left Leg & Day Time (Morning) \\
\hline V1. Observation 4a & No & Left Leg & Night Time (during sleep) \\
\hline V1. Observation 4b & No & Left Leg & Morning Time (during sleep) \\
\hline V1. Observation 4c & No & Left Leg & Evening \\
\hline V1. Observation 5 & Yes & Right Leg & Day Time (Morning) \\
\hline V1. Observation 5a & No & Right Leg & Evening \\
\hline V1. Observation 5b & No & Right Leg & Evening \\
\hline V1. Observation 5c & No & Right Leg & Night Time (during sleep) \\
\hline
\end{tabular}

\section{Results}

V1. Observation 1: Seizure occurs with sensor positioned on right wrist

Observation Time: $20.37-39 \mathrm{pm}$

Seizure Occurrence Time: $20.38 \mathrm{pm}$

V1. Observation 1

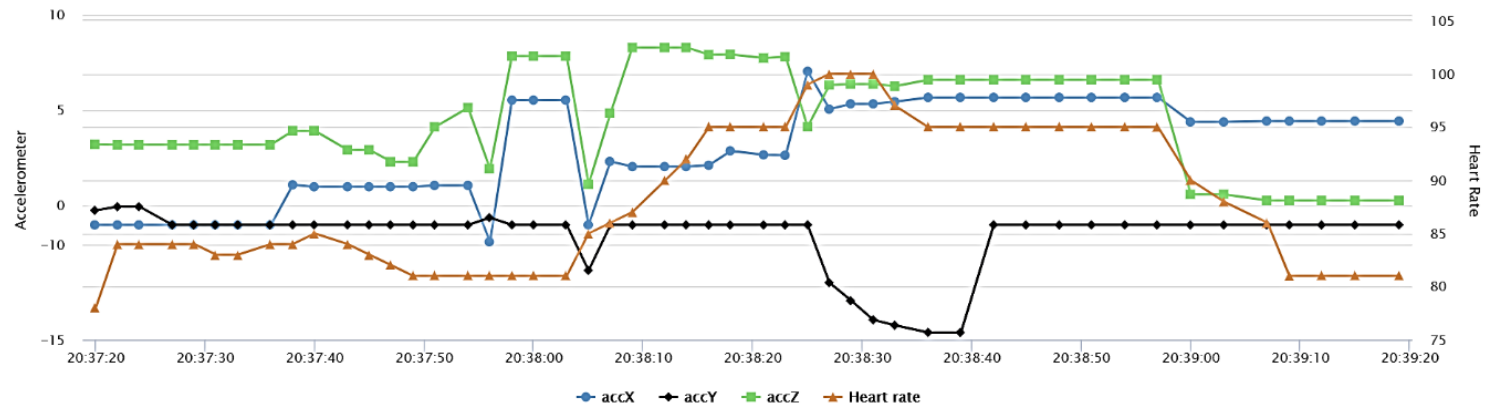


V1. Observation 2a: No seizure occurs at night (during sleep) sensor positioned on right wrist

Observation Time: $02.49-51 \mathrm{am}$

Seizure Occurrence: No

V1. Observation $2 \mathrm{a}$

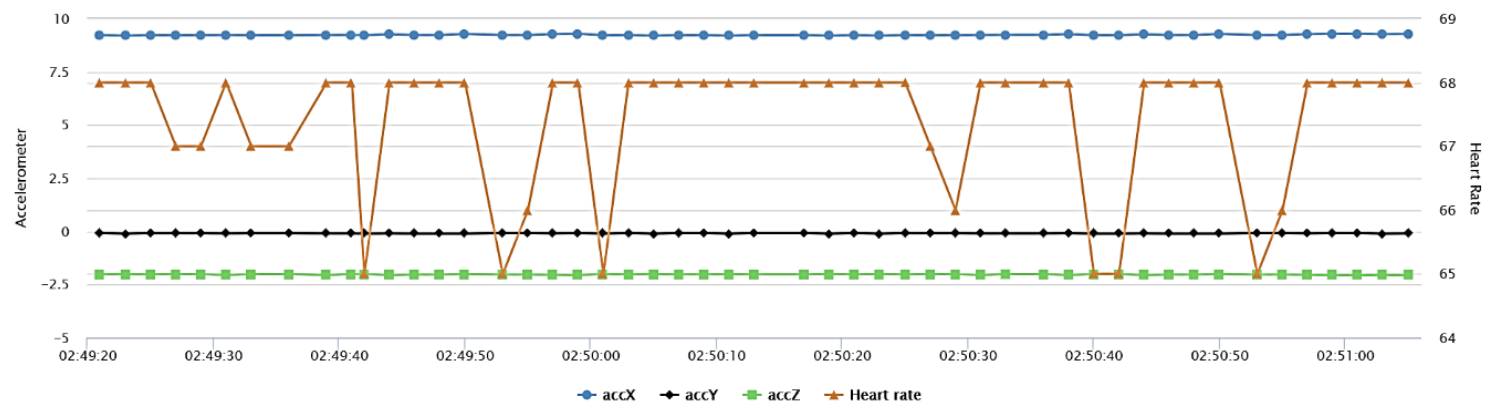

V1. Observation 2b: No seizure occurs during the evening sensor positioned on right wrist

Observation Time: $21.01-21.04$

\section{Seizure Occurrence: No}

V1. Observation $2 b$

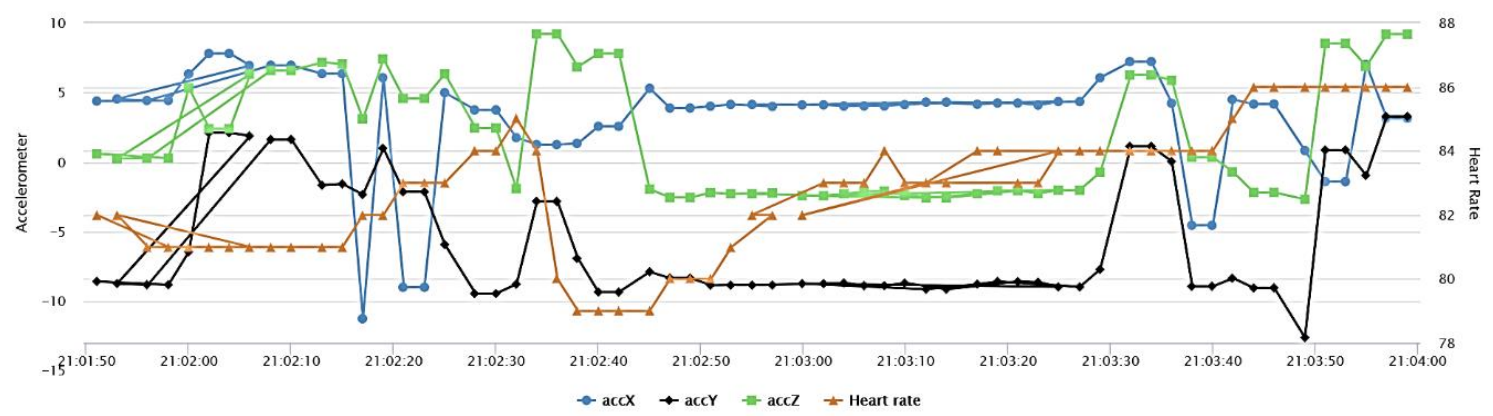

V1. Observation 2c: No seizure occurs during the evening sensor positioned on right wrist Observation Time: $19.35 \mathrm{pm}-19.37 \mathrm{pm}$

Seizure Occurrence: No

V1. Observation 2c

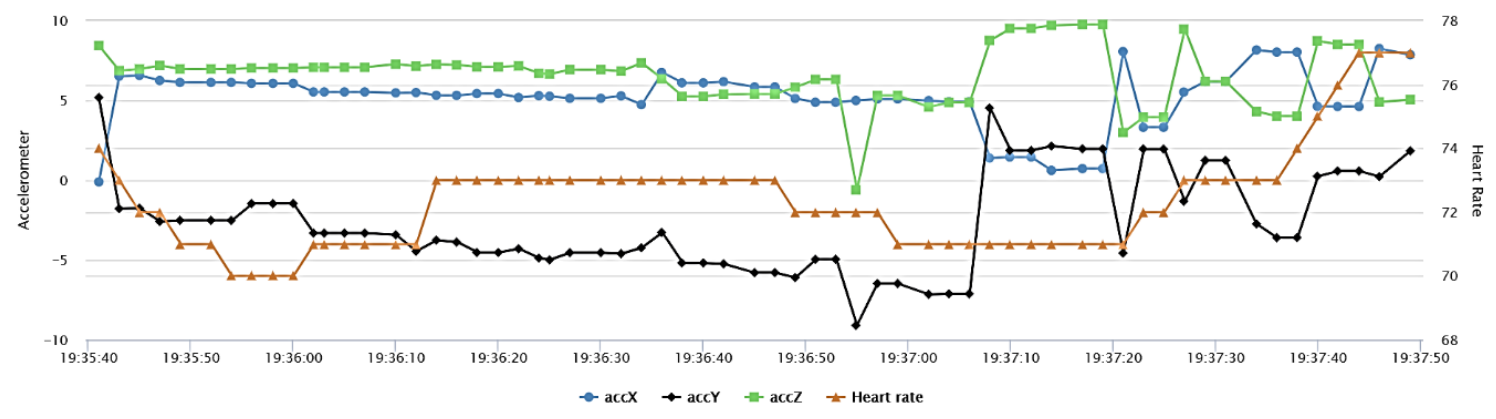


V1. Observation 3: Seizure occurs with sensor positioned on left wrist (day time)

Observation Time: 11.09-11.11am

Seizure Occurrence Time: 11.10am

V1. Observation 3

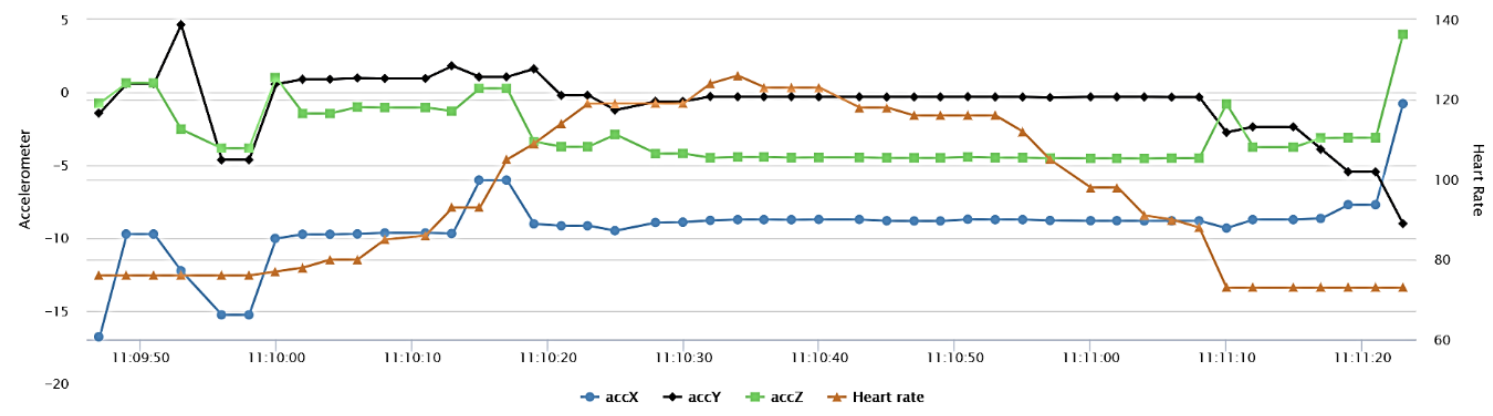

V1. Observation 3a: No Seizure occurs with sensor positioned on left wrist (night time)

Observation Time: $21.26 \mathrm{pm}-21.28 \mathrm{pm}$

Seizure Occurrence Time: None

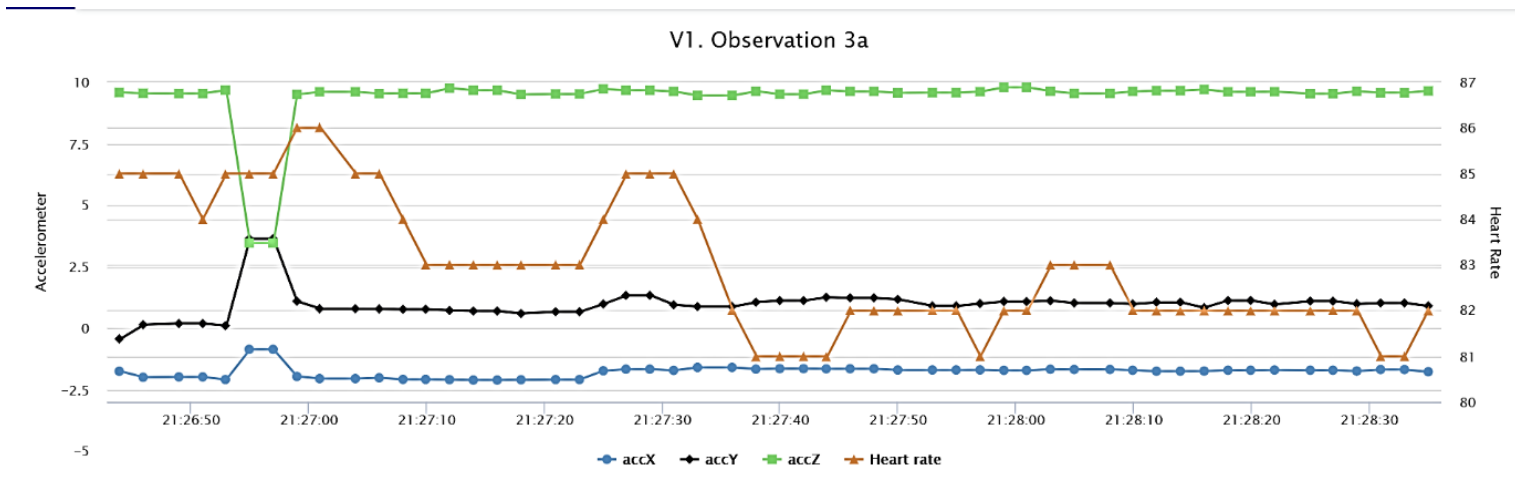

V1. Observation 3b: No Seizure occurs with sensor positioned on left wrist (morning)

Observation Time: $11.31 \mathrm{pm}$ - 11.33am

Seizure Occurrence Time: None

V1. Observation $3 b$

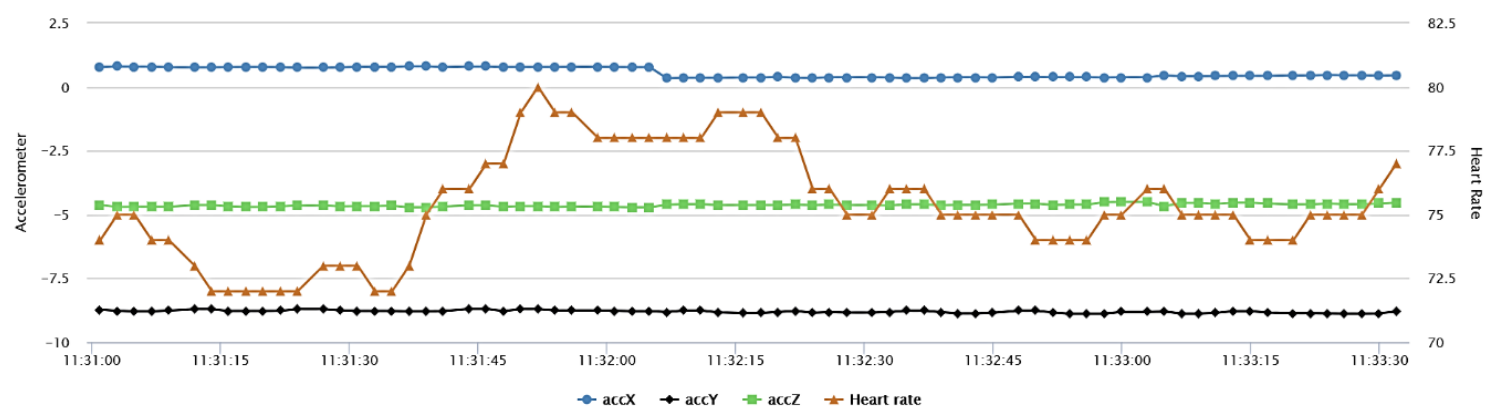


V1. Observation 3c: No Seizure occurs with sensor positioned on left wrist (evening)

Observation Time: 19.12pm - 19.15pm

Seizure Occurrence Time: None

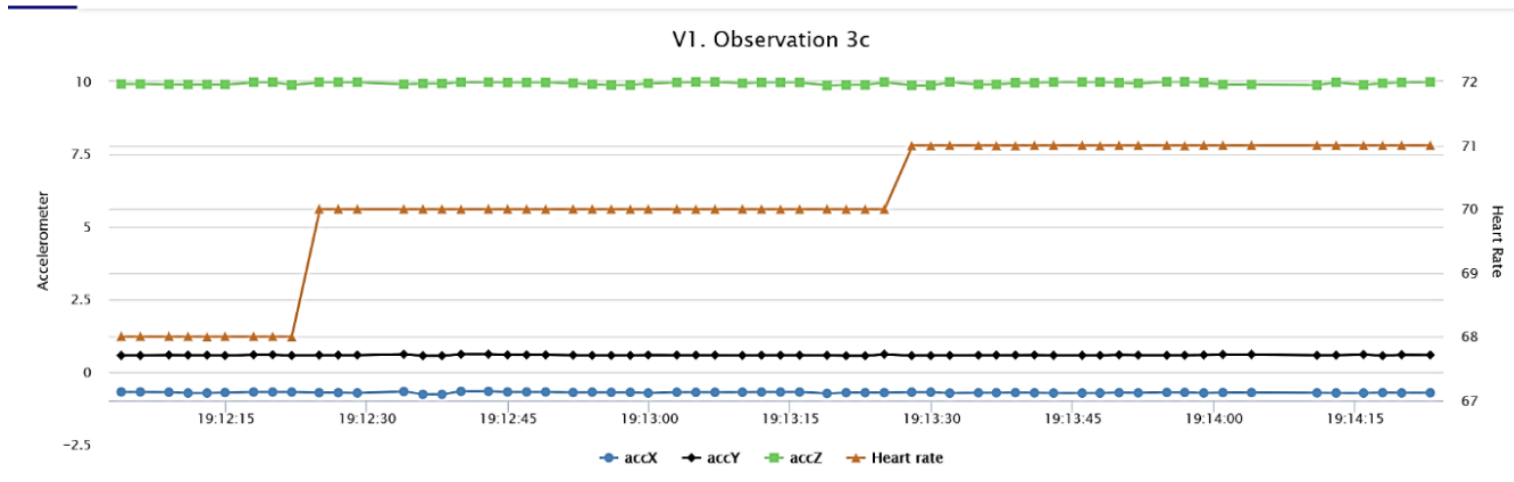

V1. Observation 4: Seizure occurs with sensor positioned on left leg (day time)

Observation Time: 09.46am - 09.48am

Seizure Occurrence Time: 09.47am

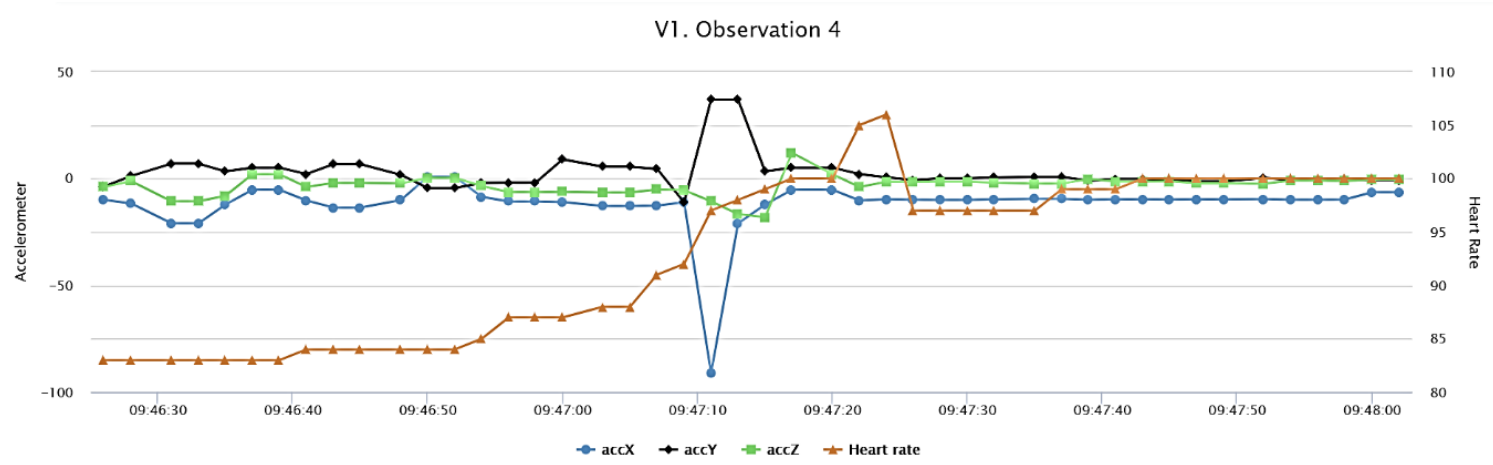

V1. Observation 4a: No seizure occurs with sensor positioned on left leg (night time, during sleep)

Observation Time: $02.15 \mathrm{am}-02.17 \mathrm{am}$

Seizure Occurrence Time: None

V1. Observation $4 a$

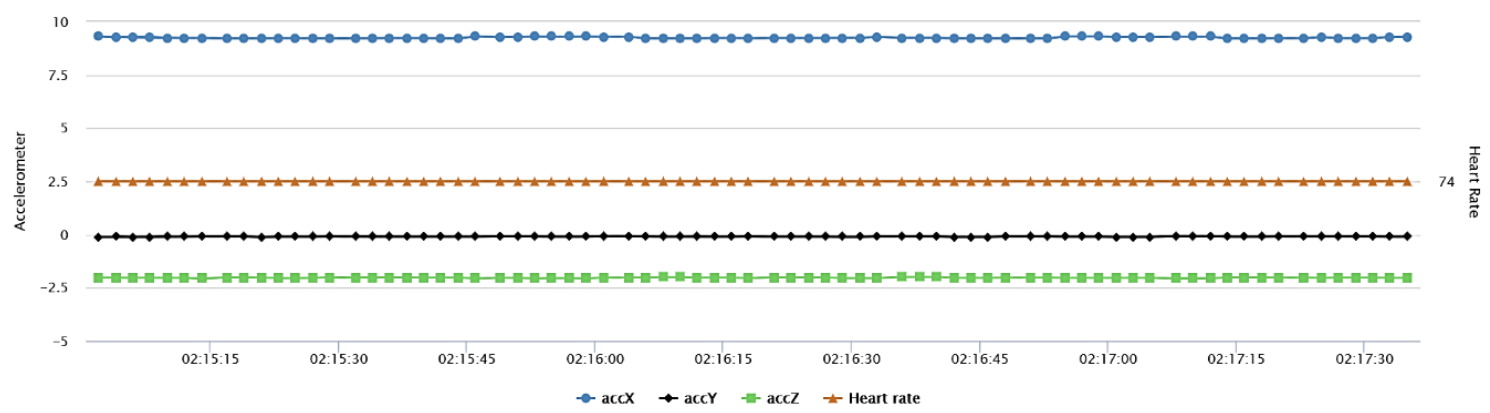


V1. Observation 4b: No seizure occurs with sensor positioned on left leg (morning, during sleep)

Observation Time: 08.10am - 08.11am

Seizure Occurrence Time: None

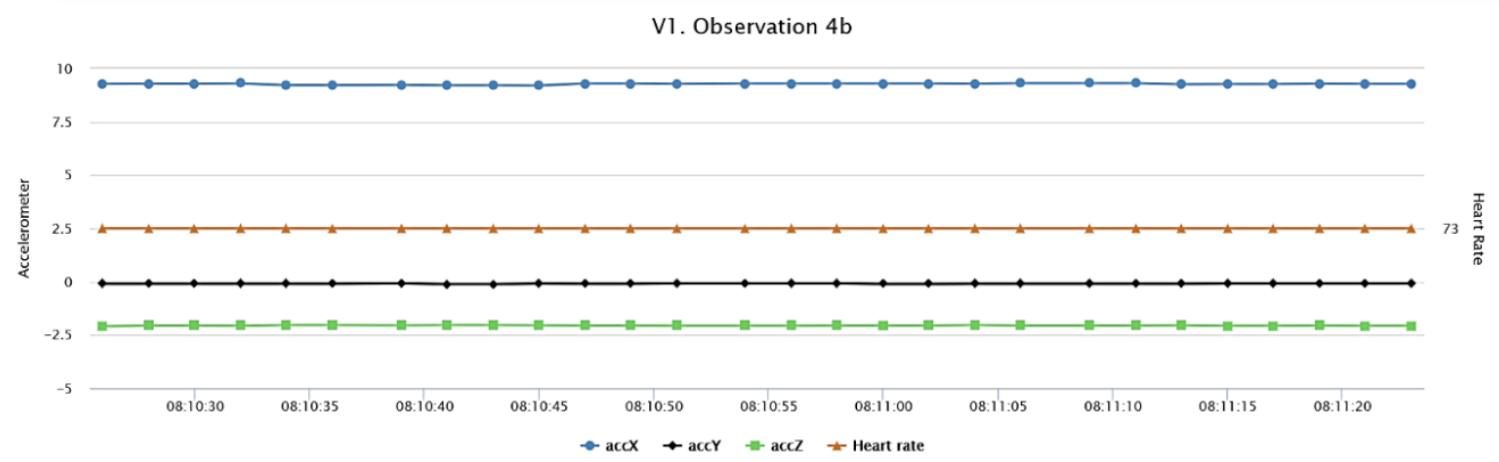

V1. Observation 4c: No seizure occurs with sensor positioned on left leg (evening)

Observation Time: $20.09-20.10 \mathrm{pm}$

Seizure Occurrence Time: None

V1. Observation $4 c$

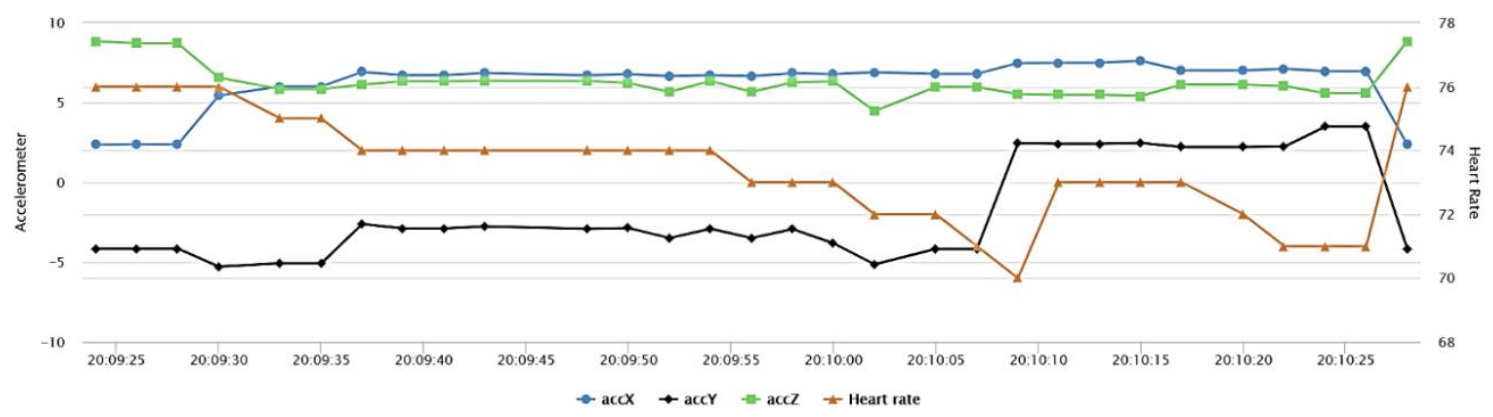

V1. Observation 5: Seizure occurs with sensor positioned on right leg (day time)

Observation Time: 11.37am - 11.40am

Seizure Occurrence Time: $11.39 \mathrm{am}$

V1. Observation 5

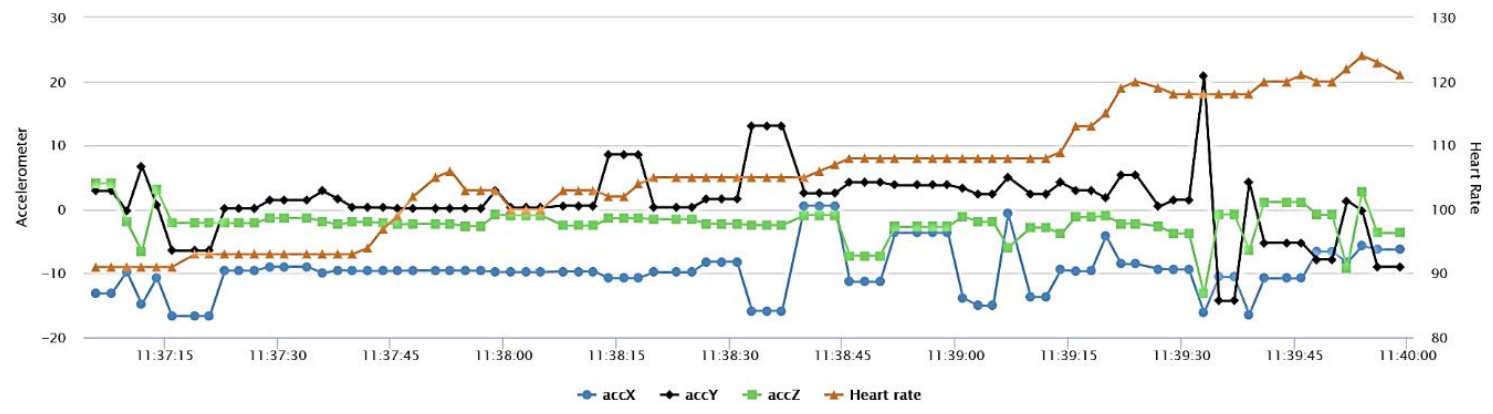


V1. Observation 5a: No seizure occurs with sensor positioned on right leg (day time)

Observation Time: $19.35 \mathrm{pm}-19.37 \mathrm{pm}$

Seizure Occurrence Time: None

V1. Observation $5 a$

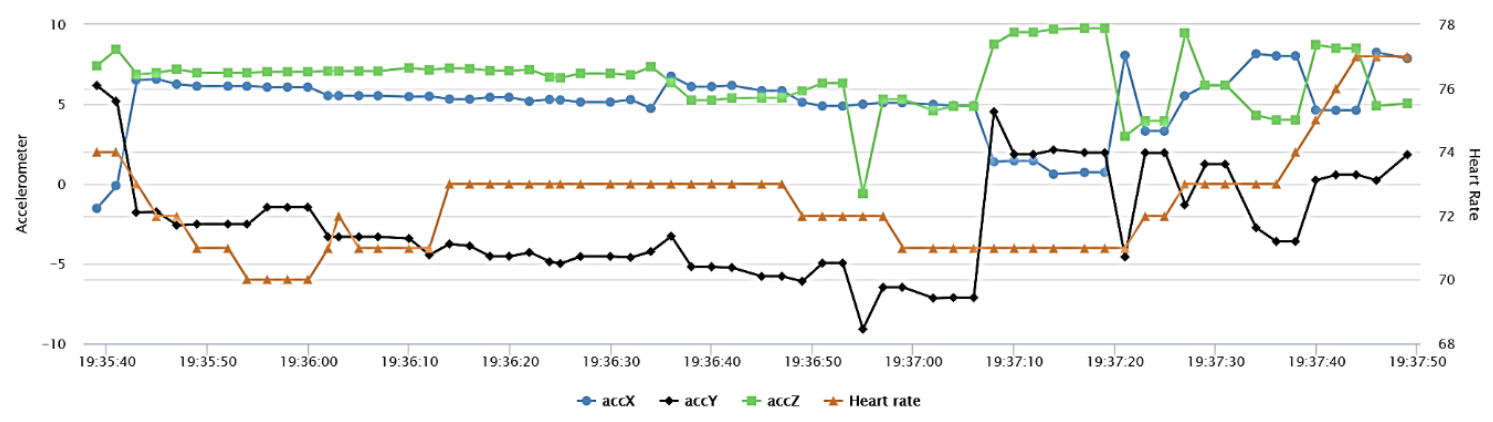

V1. Observation 5b: No seizure occurs with sensor positioned on right leg (evening)

Observation Time: $20.47-20.48 \mathrm{pm}$

Seizure Occurrence Time: None

V1. Observation $5 b$

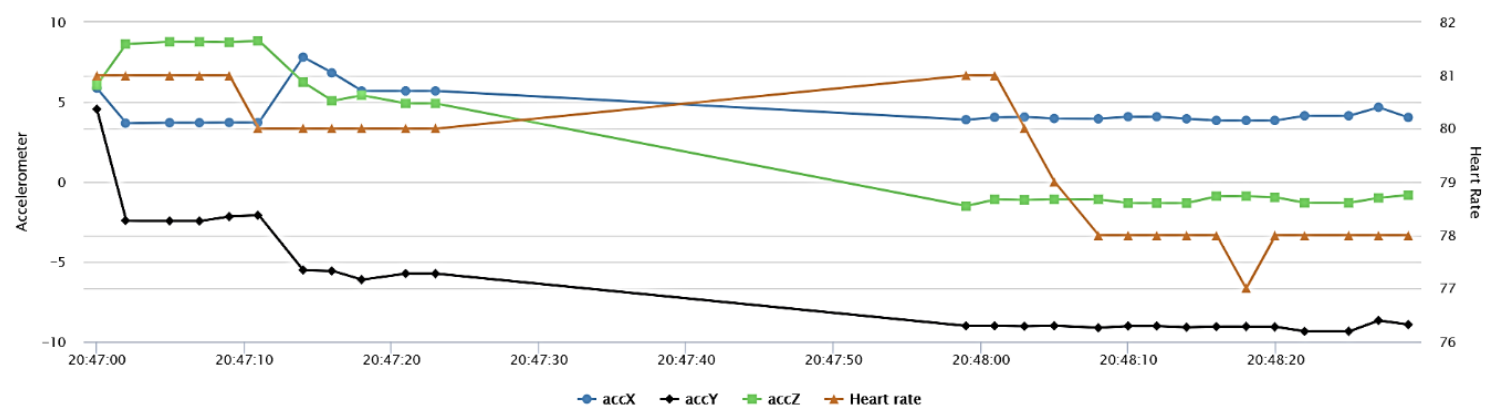

V1. Observation 5c: No seizure occurs with sensor positioned on right leg (during sleep)

Observation Time: $04.02-04.04 a m$

Seizure Occurrence Time: None

V1. Observation $5 \mathrm{c}$

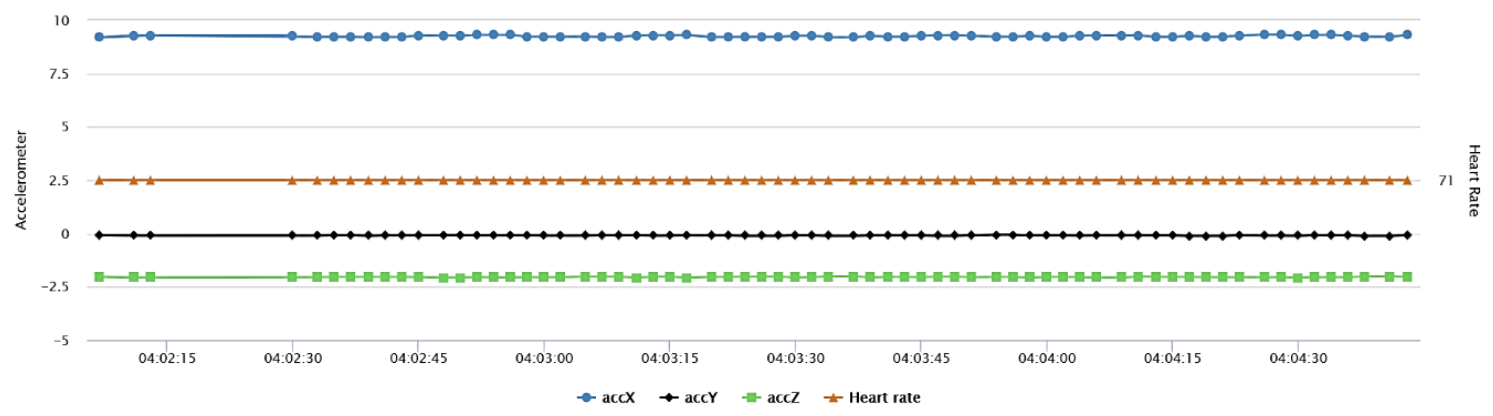




\section{Volunteer 2 Information}

\begin{tabular}{|l|l|}
\hline Volunteer Profile: & V2 \\
\hline Seizure Type: & GTCS \\
\hline Dominant Side: & Left side \\
\hline Time of Day: & Diurnal \\
\hline Trigger: & Sleep Dep \\
\hline Key Symptoms: & $\begin{array}{l}\text { Shaking left side, left leg raised then convulsion, } \\
\text { LOC }\end{array}$ \\
\hline
\end{tabular}

\section{Observation Criteria Volunteer 2}

\begin{tabular}{|l|l|l|l|}
\hline Observation & Seizure & Position & Time of Day (Random) \\
\hline V2. Observation 1 & Yes & Left Leg & Evening \\
\hline V2. Observation $1 a$ & Yes & Right Leg & Evening \\
\hline V2. Observation $1 b$ & No & Right Leg & During the evening \\
\hline V2. Observation $1 c$ & No & Left Leg & During sleep \\
\hline V2. Observation 2 & Yes & Right Leg & Day Time (Morning) \\
\hline V2. Observation $2 a$ & No & Right Leg & During sleep \\
\hline V2. Observation $2 b$ & No & Right Leg & Evening \\
\hline V2. Observation $2 c$ & No & Left Leg & Evening \\
\hline V2. Observation 3 & Yes & Left wrist & Day Time (Morning) \\
\hline V2. Observation $3 a$ & No & Left wrist & Evening \\
\hline V2. Observation $3 b$ & No & Left wrist & Afternoon \\
\hline V2. Observation $3 c$ & No & Left wrist & During Sleep \\
\hline V2. Observation 4 & Yes & Right wrist & Evening \\
\hline V2. Observation $4 a$ & No & Right wrist & During Sleep \\
\hline V2. Observation $4 b$ & No & Right wrist & Evening \\
\hline V2. Observation $4 c$ & No & Right wrist & Morning \\
\hline
\end{tabular}

\section{Results}

V2. Observation 1: Seizure occurs with sensor positioned on left leg Observation Time: $22.13-17 \mathrm{pm}$

Seizure Occurrence: $22.14 \mathrm{pm}$

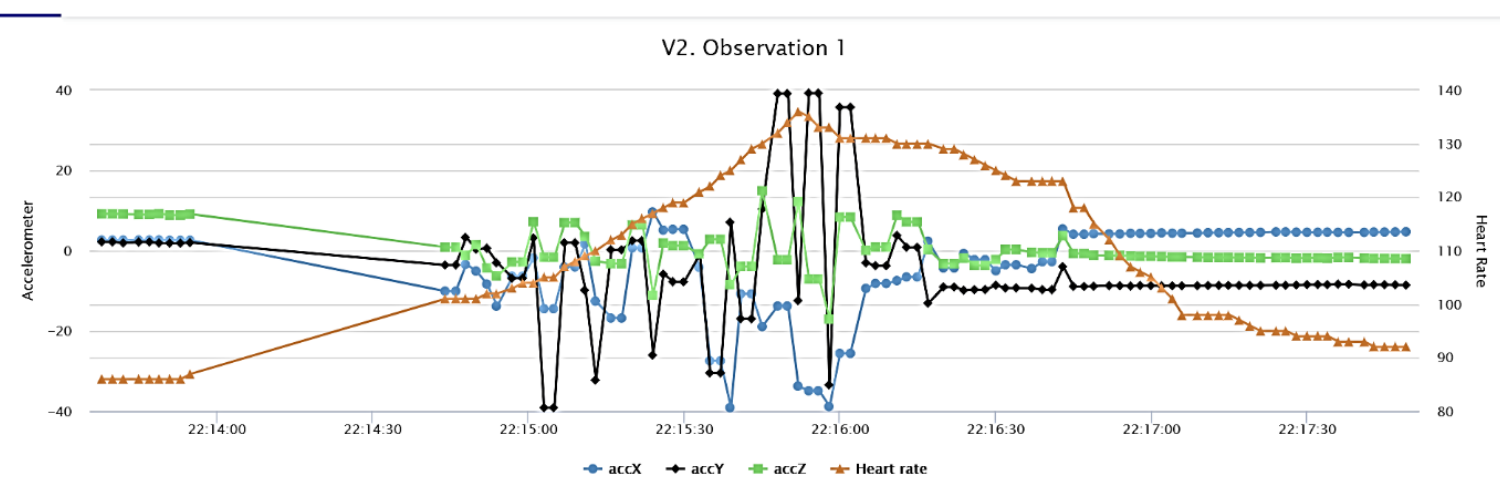


V2. Observation 1a: Seizure occurs during evening sensor positioned on right leg Observation Time: $19.31-19.32 \mathrm{pm}$

\section{Seizure Occurrence:}

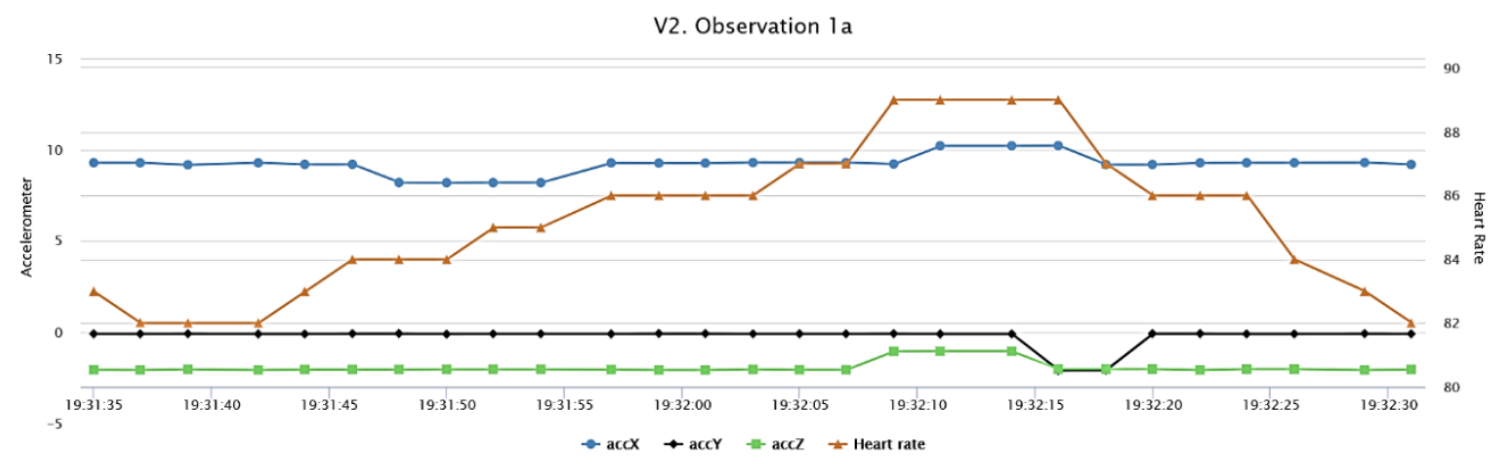

V2. Observation 1b: No seizure occurs during evening sensor positioned on right leg Observation Time: $19.35-19.36 \mathrm{pm}$

Seizure Occurrence: No seizure

V2. Observation $1 \mathrm{~b}$

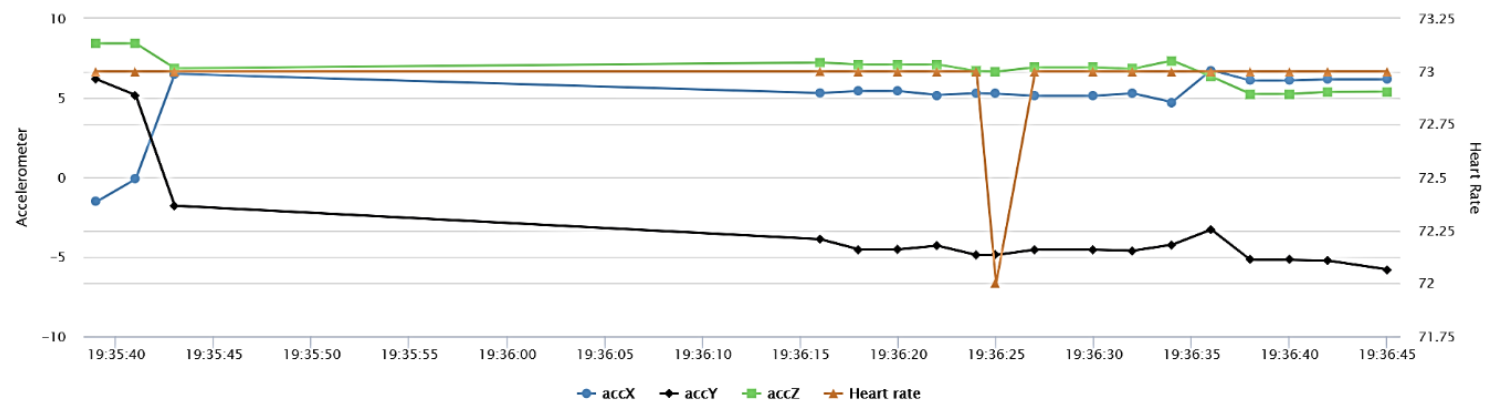

V2. Observation 1c: No seizure occurs during sleep sensor positioned on left leg Observation Time: 06.23-06.24am

Seizure Occurrence: No seizure

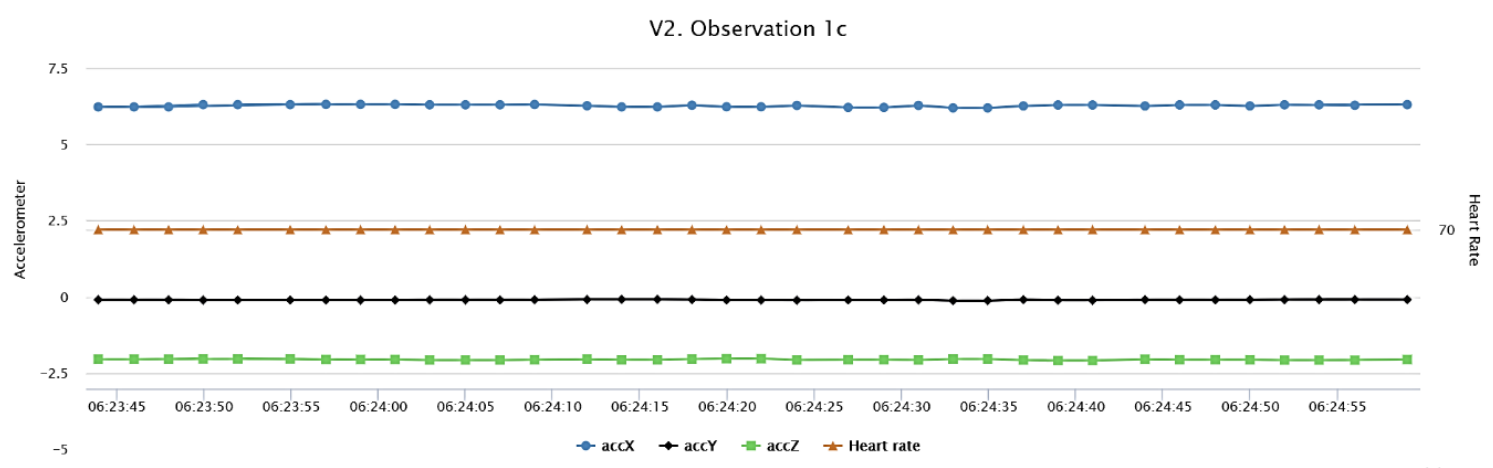


V2. Observation 2: Seizure occurs (morning) sensor positioned on right leg

Observation Time: 11.39-11.41am

Seizure Occurrence: $11.40 \mathrm{am}$

V2. Observation 2

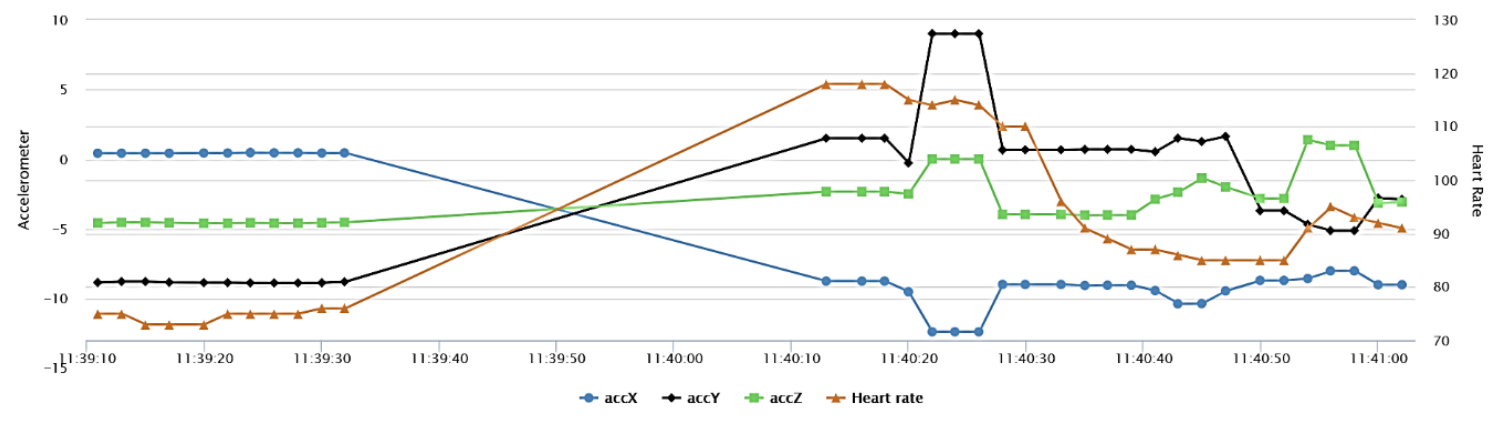

V2. Observation 2a: No seizure occurs during sleep sensor positioned on right leg

Observation Time: 02.15-02.17am

Seizure Occurrence: No Seizure

V2. Observation 2a

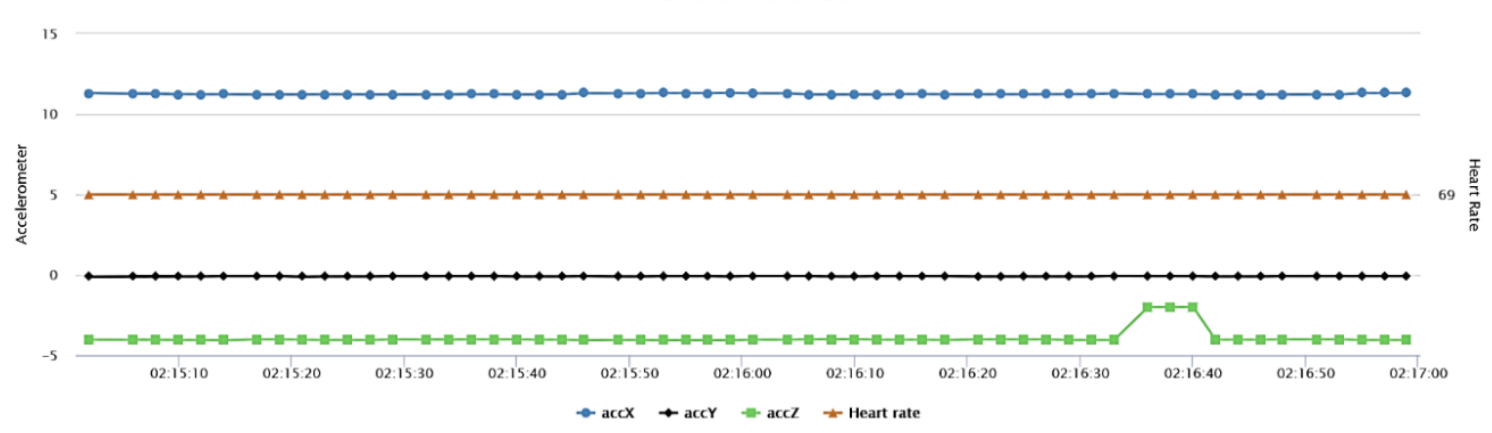

V2. Observation 2b: No seizure occurs during evening sensor positioned on right leg

Observation Time: $20.20-20.21 \mathrm{pm}$

Seizure Occurrence: No Seizure

V2. Observation $2 b$

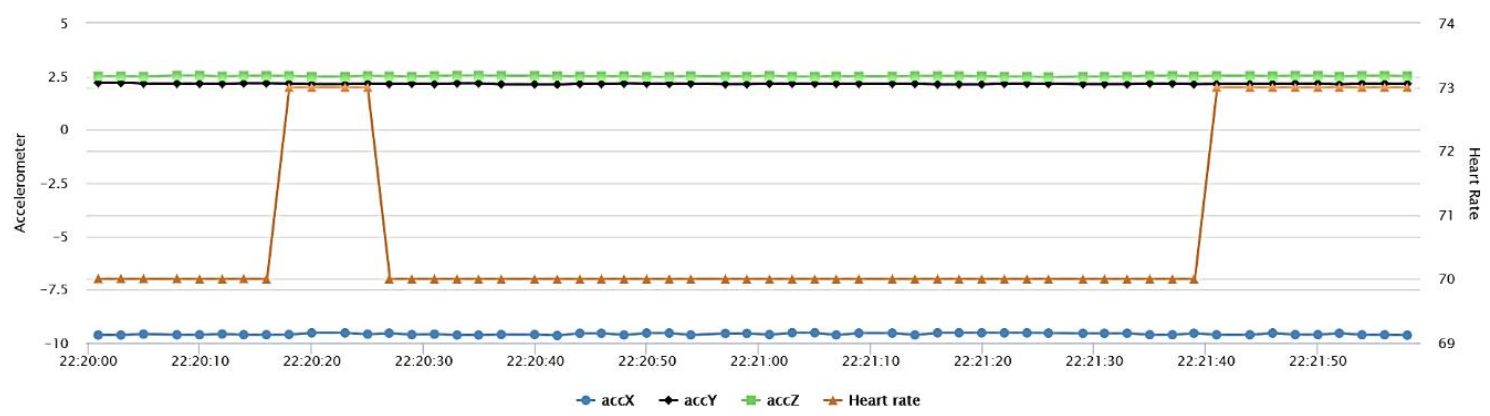


V2. Observation 2c: No seizure occurs during evening sensor positioned on left leg Observation Time: $20.34-20.35 \mathrm{pm}$

Seizure Occurrence: No Seizure

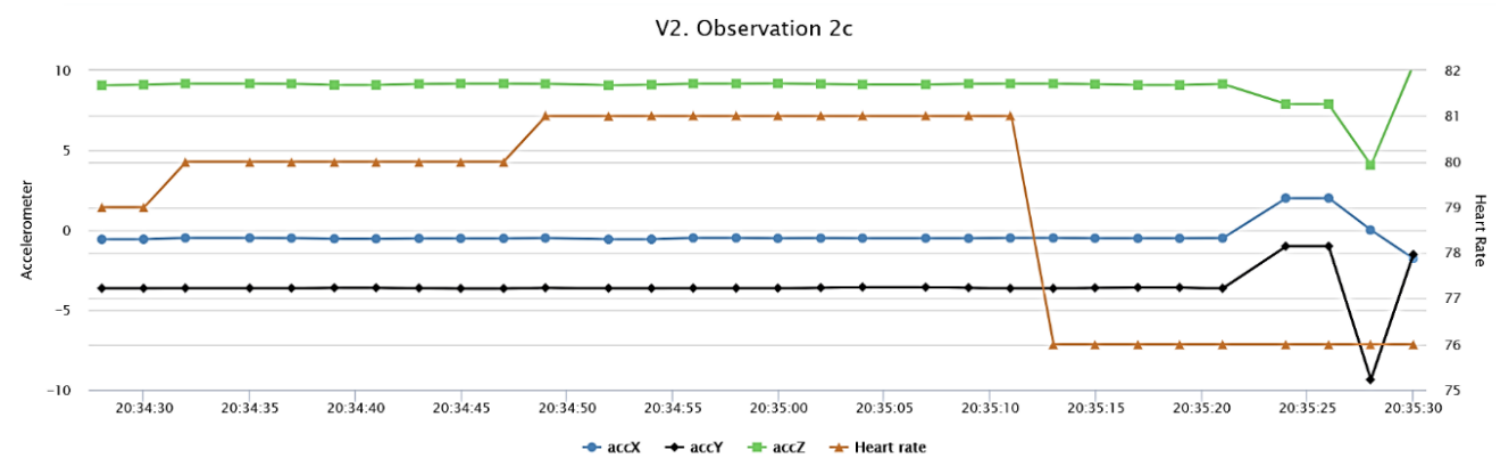

V2. Observation 3: Seizure occurs during on left wrist

Observation Time: 09.03-09.04am

Seizure Occurrence: 09.03am

V2. Observation 3

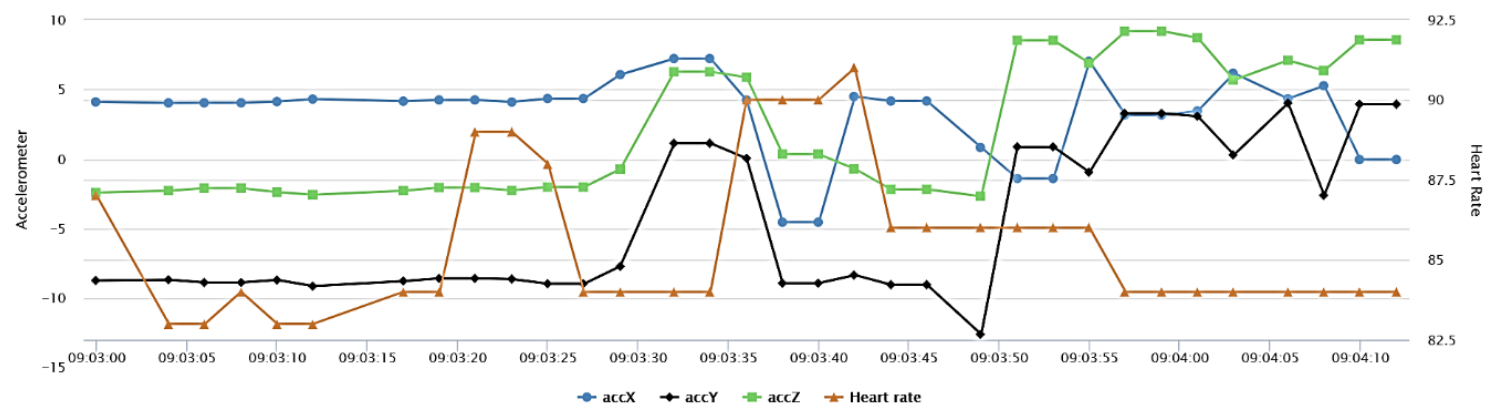

V2. Observation 3a: No seizure occurs during on left wrist

Observation Time: $20.34-20.35 \mathrm{pm}$

Seizure Occurrence:

V2. Observation 3a

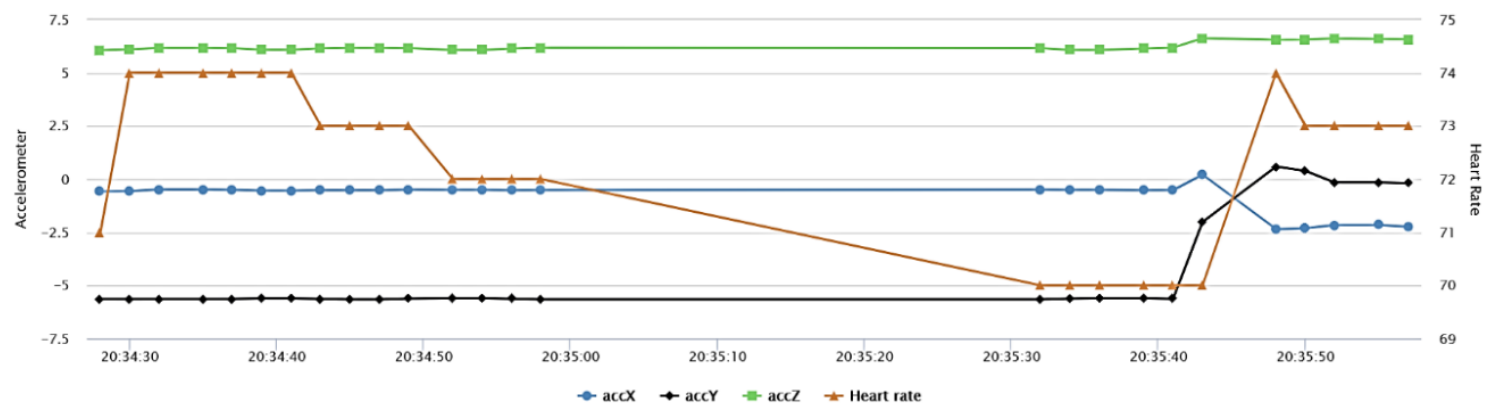


V2. Observation 3b: No seizure occurs during on left wrist

Observation Time: $13.27-13.29 \mathrm{pm}$

Seizure Occurrence: No Seizure

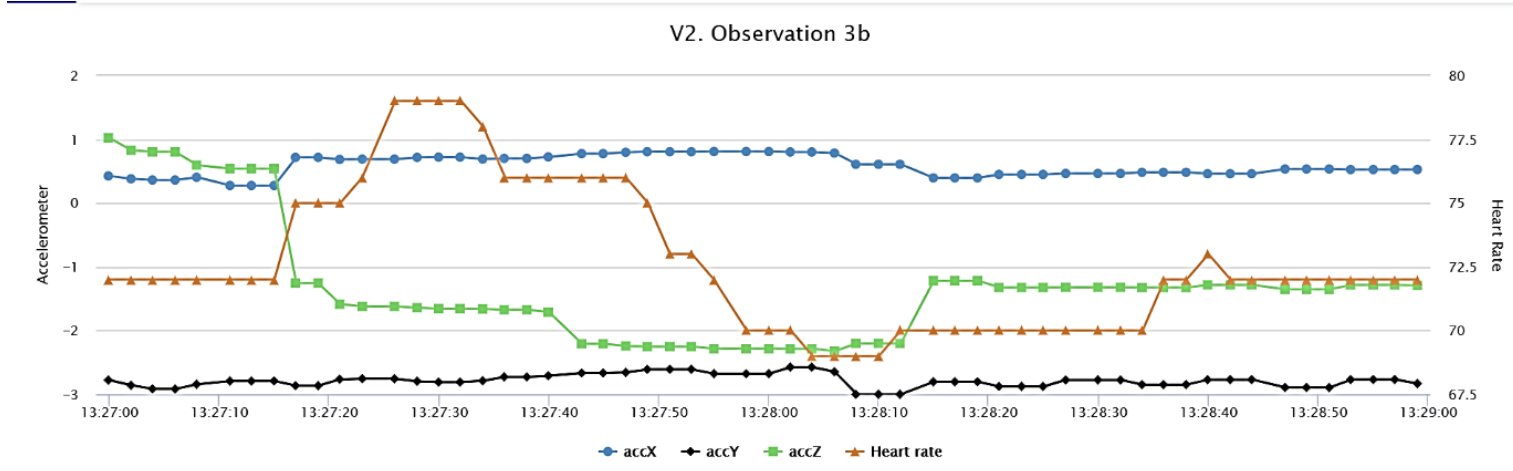

V2. Observation 3c: No seizure occurs during on left wrist (during sleep)

Observation Time: 05.15-05.17am

Seizure Occurrence: No Seizure

V2. Observation $3 c$

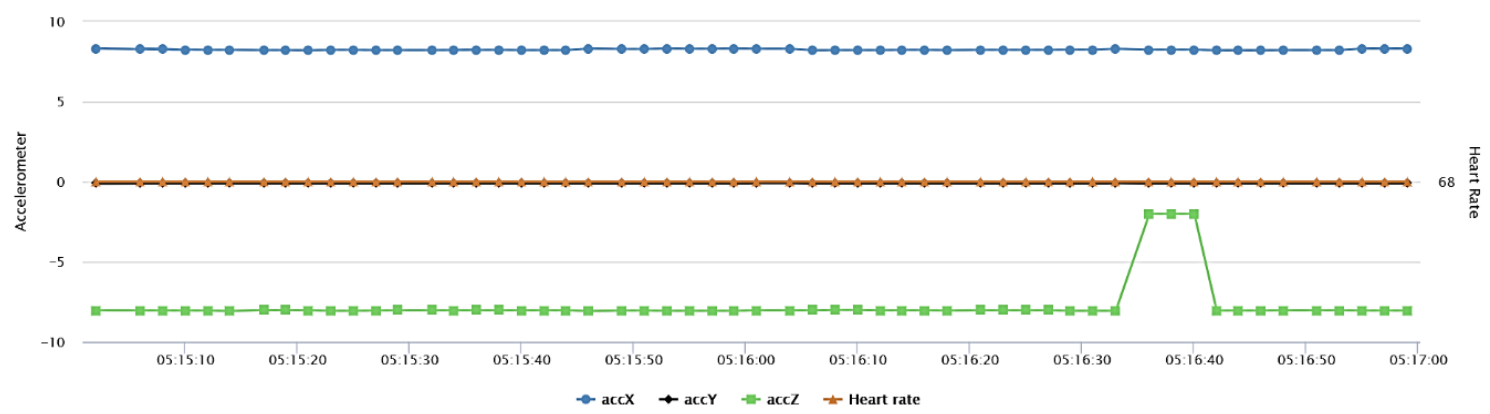

V2. Observation 4: Seizure occurs during on right wrist (evening)

Observation Time: $18.45-18.47 \mathrm{pm}$

Seizure Occurrence: 18.46

V2. Observation 4

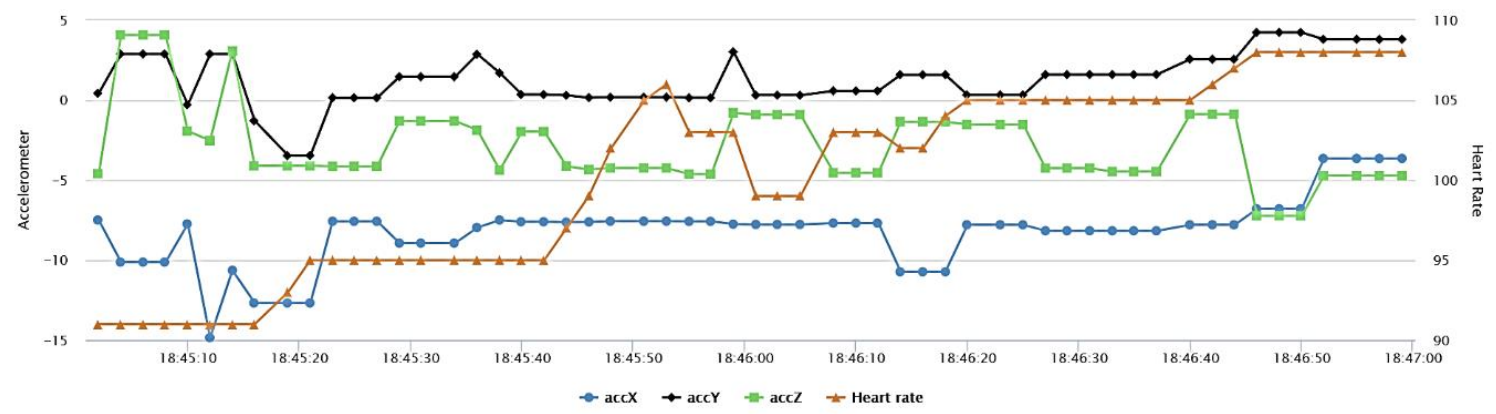


V2. Observation 4a: No seizure occurs during on right wrist (during sleep)

Observation Time: 01.02-01.04am

Seizure Occurrence: No Seizure

V2. Observation $4 a$

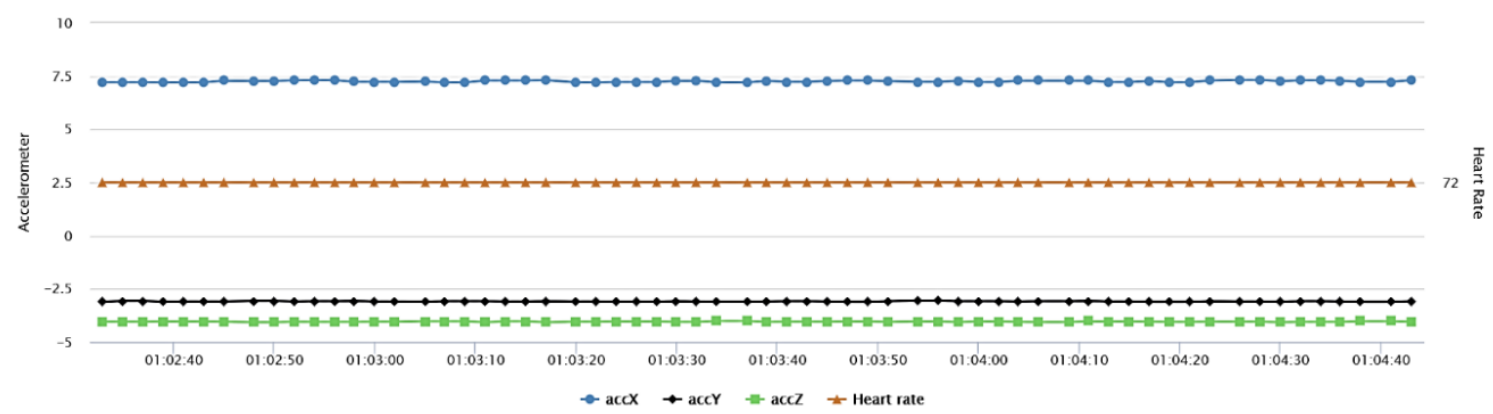

V2. Observation 4b: No seizure occurs during on right wrist (evening)

Observation Time: $21.50-21.52 \mathrm{pm}$

Seizure Occurrence: No Seizure

V2. Observation 4b

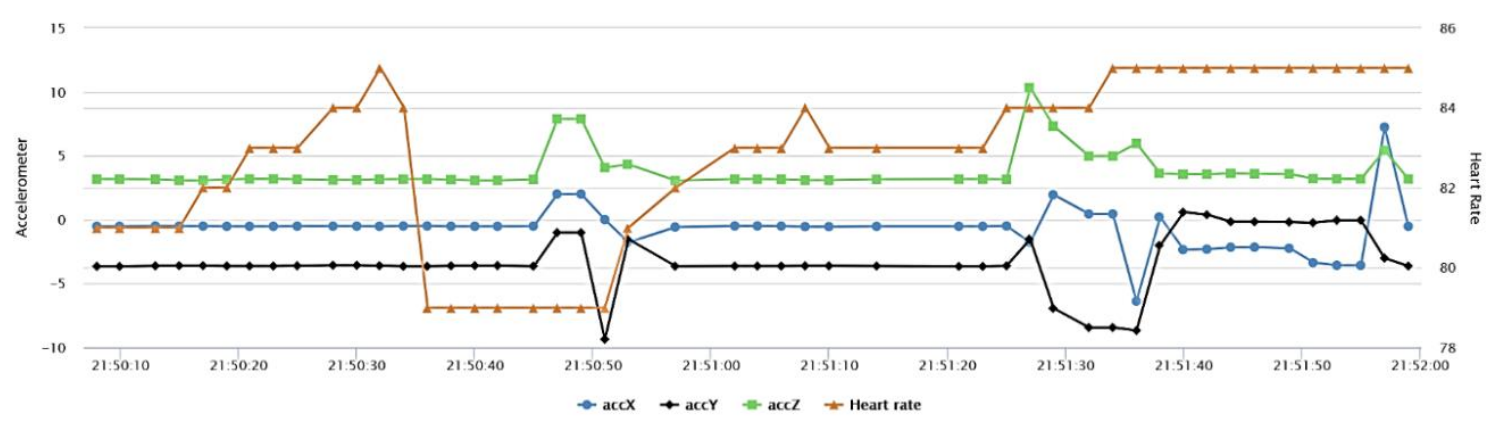

V2. Observation 4c: No seizure occurs during on right wrist (morning)

Observation Time: 10.02-10.04am

Seizure Occurrence: No Seizure

V2. Observation 4c

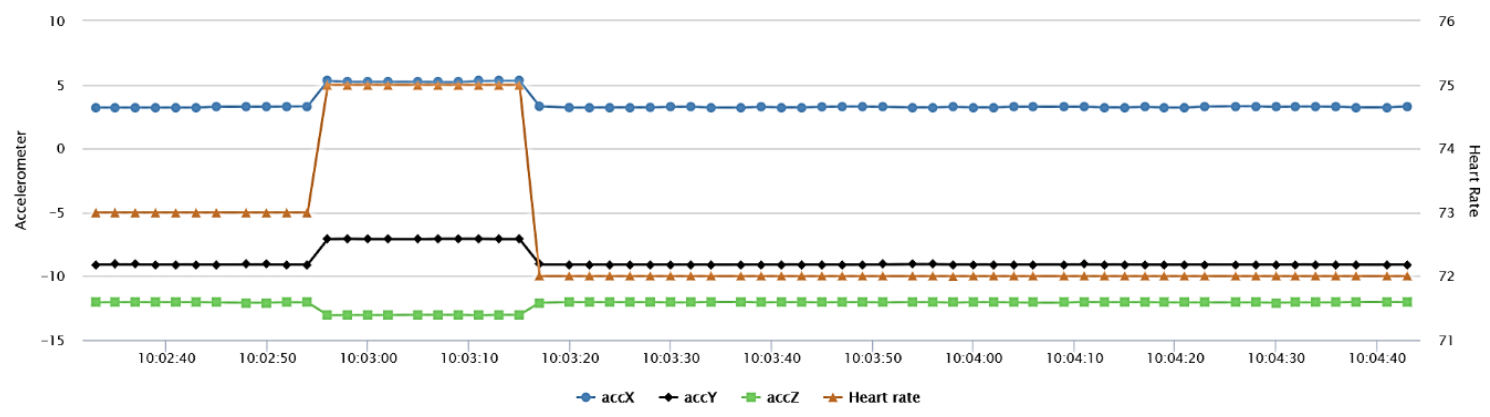

